\title{
Electronic structure and phase stability of strongly correlated electron materials
}

\author{
Eric B. Isaacs
}

Submitted in partial fulfillment of the requirements for the degree of Doctor of Philosophy in the Graduate School of Arts and Sciences

COLUMBIA UNIVERSITY

2016 
(c) 2016

Eric B. Isaacs

All Rights Reserved 


\begin{abstract}
Electronic structure and phase stability of strongly correlated electron materials
\end{abstract}

\author{
Eric B. Isaacs
}

In this thesis, we use first-principles methods to study a class of systems known as strongly correlated materials in which exceptionally strong electron-electron repulsion in the $d$ or $f$ electron shell can lead to intriguing physical properties. The focus is on transition metal oxide and phosphate intercalation materials such as $\mathrm{Li}_{x} \mathrm{CoO}_{2}$ and $\mathrm{Li}_{x} \mathrm{FePO}_{4}$, which are employed as the positive electrode in rechargeable Li ion batteries. We also study the transition metal dichalcogenide system $\mathrm{VS}_{2}$ as a candidate for strong correlation physics with analogous features to the cuprate high-temperature superconductors.

Density functional theory (DFT), the standard theory of materials science which can be viewed as an effective single-electron theory, often breaks down for strongly correlated materials. In this thesis, we augment DFT with a more sophisticated many-electron approach known as dynamical mean-field theory (DMFT). We use the resultant DFT+DMFT approach with the numerically exact continuous-time quantum Monte Carlo solver to explore the physics of the materials studied here and probe compositional phase stability and related observables within DFT+DMFT for the first time. The elementary but efficient Hartree-Fock solver for the DMFT equations (i.e., DFT $+U$ ) is also utilized in order to cleanly separate the role of dynamical correlations and to better understand the respective methods. With these $a b$ initio methods, we predict the compositional phase stability, average intercalation voltage, Li order-disorder transition temperature, structural phase stability, phonons, magnetic 
properties, and other important characteristics of strongly correlated materials.

At the $\mathrm{DFT}+U$ level of theory, electronic correlations destabilize the intermediate- $x$ compounds of cathode materials via enhanced ordering of the endmember $d$ orbitals. DFT $+U$ is qualitatively consistent with experiments for phase stable $\mathrm{Li}_{x} \mathrm{CoO}_{2}$, phase separating $\mathrm{Li}_{x} \mathrm{FePO}_{4}$, and phase stable $\mathrm{Li}_{x} \mathrm{CoPO}_{4}$. In $\mathrm{Li}_{1 / 2} \mathrm{CoO}_{2}$, which is not charge ordered in experiments, the charge ordering predicted by $\mathrm{DFT}+U$ primarily stems from the approximate interaction, is necessary to qualitatively capture the phase stability, and erroneously predicts an insulating state and an overestimated Li order-disorder transition temperature. DFT+DMFT calculations describe $\mathrm{LiCoO}_{2}$ as a band insulator with appreciable correlations within the $E_{g}$ states and $\mathrm{CoO}_{2}$ as a moderately correlated Fermi liquid; for both these systems we find evidence for appreciable charge and spin fluctuations. Dynamical correlations substantially dampen changes in the number of $d$ electrons per site and the total energy as compared to $\mathrm{DFT}+U$, which alters the predicted battery voltage between the two methods. We find that our DFT+DMFT results underestimate the average intercalation voltage for $\mathrm{Li}_{x} \mathrm{CoO}_{2}$ and discuss possible reasons for the discrepancy.

In monolayer $\mathrm{VS}_{2}$, a combination of crystal field splitting and direct $\mathrm{V}-\mathrm{V}$ hopping leads to an isolated low-energy band for the trigonal prismatic phase within non-spin-polarized DFT. Ferromagnetism spin splits this band within spin DFT and leads to a $S=1 / 2$ ferromagnetic Stoner insulator. DFT $+U$ opens this gap and leads to Mott insulating behavior, though for sufficiently high $U$ an octahedral phase becomes favored. Using the known charge density wave of this octahedral phase, we assess the validity of DFT and DFT $+U$ in this class of materials. If realized, trigonal prismatic $\mathrm{VS}_{2}$ could be experimentally probed in an unprecedented fashion due to its monolayer nature. 


\section{Contents}

List of Tables $\quad$ iv

List of Figures $\quad$ v

1 Introduction $\quad 1$

1.1 The many-body problem in electronic structure . . . . . . . . . . . 1

1.2 Density functional theory . . . . . . . . . . . . . . . . 3

1.3 Strongly correlated materials . . . . . . . . . . . . . . . . . . 6

1.3.1 Hubbard model . . . . . . . . . . . . . . . . 7

1.4 DFT plus Hubbard $U \ldots \ldots \ldots$

1.4 .1 Basic form . . . . . . . . . . . . . . . 10

1.4.2 Rotationally-invariant formalism . . . . . . . . . . . . . 16

1.5 DFT plus dynamical mean-field theory . . . . . . . . . . . . . . . . 17

1.5.1 Dynamical mean-field theory . . . . . . . . . . . . . . . . . 17

1.5.2 Merging DFT and dynamical mean-field theory . . . . . . . . . . 21

2 Phase stability of strongly correlated electron materials within DFT $+U \quad 28$

2.1 Introduction . . . . . . . . . . . . . . . . . . . . . . . 29

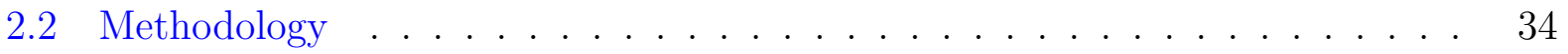

2.2.1 DFT $+U$ approach for correlated materials . . . . . . . . . . . 34

2.2.2 Filling and ordering energy decomposition in $\mathrm{DFT}+U \ldots$. . . . . . 36

2.3 Computational Details . . . . . . . . . . . . . . . . . . . . 39

2.4 Results and Discussion . . . . . . . . . . . . . . . . . . 40 
2.4.1 Endmember electronic structure within DFT . . . . . . . . . . . . . 40

2.4.2 Impact of $U$ on electronic structure of $\mathrm{Li}_{x} \mathrm{CoO}_{2} \quad \ldots \ldots \ldots$

2.4.3 Tendency for charge ordering in $\mathrm{DFT}+U \ldots \ldots$. . . . . . . . . 44

2.4.4 Impact of $U$ on phase stability of $\mathrm{Li}_{x} \mathrm{CoO}_{2} \ldots \ldots \ldots \ldots$

2.4.5 Impact of $U$ on electronic structure of $\mathrm{Li}_{x} \mathrm{FePO}_{4} \ldots \ldots \ldots \ldots$. . . 54

2.4.6 Impact of $U$ on phase stability of $\mathrm{Li}_{x} \mathrm{FePO}_{4} \ldots \ldots \ldots \ldots \ldots$

2.4.7 Phase stability of $\mathrm{Li}_{x} \mathrm{CoPO}_{4} \ldots \ldots \ldots \ldots \ldots$

2.4.8 Impact of double counting on phase separation trend $\ldots \ldots \ldots$. . . 65

2.4.9 Average intercalation voltage of $\mathrm{Li}_{x} \mathrm{CoO}_{2}$ and $\mathrm{Li}_{x} \mathrm{FePO}_{4} \ldots \ldots . .67$

2.4.10 Li order-disorder transition temperature of $\mathrm{Li}_{1 / 2} \mathrm{CoO}_{2} \ldots \ldots \ldots$

2.5 Conclusions $\ldots \ldots \ldots \ldots \ldots$

2.6 Acknowledgments . . . . . . . . . . . . . . . . . . . . . . . . . 73

3 Density functional plus dynamical mean-field theory of $\mathrm{Li}_{x} \mathrm{CoO}_{2} \quad 74$

3.1 Introduction . . . . . . . . . . . . . . . . . . . . 75

3.2 Computational Details . . . . . . . . . . . . . . . . . . . 77

3.3 Results and Discussion . . . . . . . . . . . . . . . . . . . . . 78

3.3 .1 Total energy . . . . . . . . . . . . . . . . . . . . . . 78

3.3 .2 Electronic properties . . . . . . . . . . . . . . . . . . . . 82

3.3 .3 Average intercalation voltage . . . . . . . . . . . . . . . 92

3.4 Conclusions . . . . . . . . . . . . . . . . . . . . . . . . . . . . 95

3.5 Acknowledgments . . . . . . . . . . . . . . . . . . 96

$\begin{array}{lll}4 & \text { Electronic correlations in monolayer } \mathrm{VS}_{2} & \mathbf{9 7}\end{array}$

4.1 Introduction . . . . . . . . . . . . . . . . . . . . . . . . 98

4.2 Computational Details . . . . . . . . . . . . . . . . . . . . . 102

4.3 Results and Discussion . . . . . . . . . . . . . . . . . . . . . . 103

4.3.1 Charge density wave in octahedral $\mathrm{VS}_{2}$ within DFT . . . . . . . 103 
4.3.2 Non-spin-polarized DFT electronic structure . . . . . . . . . . . . 105

4.3.3 DFT energy level diagram . . . . . . . . . . . . . . 109

4.3.4 Impact of on-site Hubbard $U \ldots \ldots$. . . . . . . . . . . 111

4.3.5 DFT $+U$ relative phase stability . . . . . . . . . . . . . 123

4.3.6 Possibility of realizing trigonal prismatic $\mathrm{VS}_{2} \ldots \ldots \ldots$. . . . . . . 127

4.4 Conclusions . . . . . . . . . . . . . . . . . . . . . . 128

4.5 Acknowledgments . . . . . . . . . . . . . . . . . . . . 129

Bibliography

147 


\section{List of Tables}

1.1 Exponential scaling of the time to solve the many-electron Schrödinger equation, assuming a single-electron problem takes 1 second to solve numerically and the scaling prefactor is unity. The time to solve a 100-electron problem $\left(10^{35}\right.$ years $)$ is astronomical. . . . . . . . . . . . . . . 2

4.1 Total energy change per formula unit with respect to the pristine structure of the same magnetic state, $\mathrm{V}-\mathrm{S}$ bond length range, and $\mathrm{V}$ magnetic moment range for the NSP and FM states of $\mathrm{OCT} \mathrm{VS}_{2}$ with $q=3 / 5 \mathrm{~K}$ and $q=2 / 3 \mathrm{~K}$ relaxed structures. . . . . . . . . . . . . . . . . . . . . . . . 113

4.2 Total energy change per formula unit, $\mathrm{V}-\mathrm{S}$ bond length range, and $\mathrm{V}$ magnetic moment range for FM TP $q=K$ and $q=3 / 5 K$ relaxed structures for several U values. . . . . . . . . . . . . . . . . . . . . . . . . . 120 


\section{List of Figures}

1.1 DFT maps an interacting $N_{\text {elec }}$-electron problem with the full interactions onto an auxiliary 1-electron problem with an effective Kohn-Sham potential containing contributions from the electron-nucleus interaction, mean-field Coulomb interaction, and the exchange-correlation potential. In this schematic, the black circles correspond to electrons and the gradient on the right side represents the fictitious Kohn-Sham potential. . . . . . . . . . . . . . .

1.2 Radial probability distribution for $\mathrm{Cu} 4 s$ (black), $\mathrm{Cu} 3 d$ (blue), and $\mathrm{F} 2 p$ based on LDA calculations of single atoms. The F $2 p$ curve is centered at $r=4 a_{B}$, where $a_{B}$ is the Bohr radius. $\mathrm{Cu}$ and $\mathrm{F}$ are in in the $[\mathrm{Ar}] 3 d^{10} 4 s^{1}$ and $[\mathrm{He}] 2 s^{2} 2 p^{5}$ configurations, respectively. Figure taken from Pavarini et al. (2011).

1.3 Schematic of the Hubbard model on a two-dimensional square lattice. Electron hopping between sites is favorable with energy $-t$. Double occupancy on a single site results in an energy penalty of $+U \ldots \ldots$. . . . . . . . .

1.4 Schematic of total energy versus number of electrons $N$ for exact (red) and approximate (black) DFT. The correction to the total energy from the DFT $+U$ method shown in blue can be viewed as a means to help restore the missing derivative discontinuity in approximate DFT. Figure taken from Cococcioni and de Gironcoli (2005). . . . . . . . . . . . . . . . . 
1.5 DMFT maps the lattice many-body problem onto a quantum impurity problem of a single lattice site embedded in a fictitious bath of electrons chosen to mimic the effect of the discarded lattice sites. DMFT, which is exact in the limit of infinite dimensions, captures the dynamical local correlations. In the right part of this schematic, a single correlated orbital is considered. As a function of time, electrons can hop on and off of the site via hybridization with the bath. The concept of this image comes from Kotliar and Vollhardt

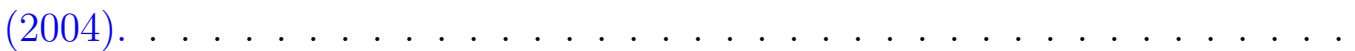

1.6 Overall workflow for the DFT+DMFT calculations. . . . . . . . . . 25

1.7 DMFT component of the workflow for DFT+DMFT calculations. . . . . . 26

2.1 Crystal structures of (a) $\mathrm{Li}_{x} \mathrm{CoO}_{2}$ and (b) $\mathrm{Li}_{x} \mathrm{FePO}_{4}$. The large green, medium blue, medium gold, small purple, and small red spheres represent ionic positions of $\mathrm{Li}, \mathrm{Co}, \mathrm{Fe}, \mathrm{P}$, and $\mathrm{O}$, respectively The black lines indicate for (a) the unit cell of the lowest-energy $\mathrm{Li}_{1 / 2} \mathrm{CoO}_{2}$ configuration and for (b) the primitive unit cell. Images of crystal structures are generated using VESTA [Momma and Izumi (2011)]. . . . . . . . . . . . . . . . . .

2.2 Electronic density of states for $\mathrm{CoO}_{2}$ in the $\mathrm{O} 3$ structure (solid black lines) and O1 structures (dashed red lines) within DFT, indicating the different layer stacking has a very small effect on the density of states. The thin dashed black line indicates the Fermi energy. . . . . . . . . . . . . . .

2.3 Projected $p$ and $d$ density of states for (a) $\mathrm{CoO}_{2}$, (b) $\mathrm{LiCoO}_{2}$, (c), $\mathrm{FePO}_{4}$, and (d) $\mathrm{LiFePO}_{4}$ within DFT. For antiferromagnetic $\mathrm{FePO}_{4}$ and $\mathrm{LiFePO}_{4}$ only a single spin channel is shown. The dashed gray lines indicate the valence band maximum for insulators and the Fermi energy for metals. Insets are the nominal transition metal $3 d$ orbital fillings from crystal field theory. . . . . . 
2.4 Electronic band gap as a function of $U$ for (a) $\mathrm{CoO}_{2}$, (b) $\mathrm{Li}_{1 / 2} \mathrm{CoO}_{2}$, and (c) $\mathrm{LiCoO}_{2}$ with relaxations (filled red squares) and with frozen $U=0$ structures (large open black circles). The corresponding plots of Co magnetic moment are shown in panels (d)-(f). For panels (b) and (e), the additional small filled blue circles correspond to calculations with frozen $U=0$ structures and $\mathrm{CO}$

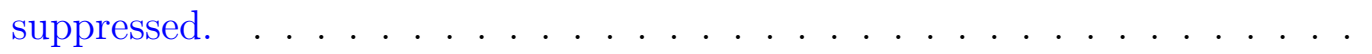

2.5 (a) Co $\mathrm{N}_{d}$, (b) Co magnetic moments, and (c) total energy for $\mathrm{Li}_{1 / 2} \mathrm{CoO}_{2}$ with the frozen $U=0 \mathrm{eV}$ structure for standard DFT $+U$ (blue lines) as well as with the interaction (filled red circles) or double counting (open black squares) terms averaged over correlated sites. Total energies in panel (c) are with respect to those of the standard $\mathrm{DFT}+U \ldots \ldots \ldots \ldots$

2.6 (a) Total $\mathrm{Li}_{1 / 2} \mathrm{CoO}_{2}$ formation energy (open black circles) and its DFT (filled red squares) and $U$-dc (filled blue circles) components as a function of $U$ (b) $\mathrm{Li}_{1 / 2} \mathrm{CoO}_{2} U$-dc (filled blue circles) formation energy component and its orbital filling (open red circles) and orbital ordering (open black squares) components as a function of $U$ (c) Number of $d$ electrons per Co as a function of $U$ for $\mathrm{CoO}_{2}$ (filled black circles), $\mathrm{Li}_{1 / 2} \mathrm{CoO}_{2}$ (open red squares), and $\mathrm{LiCoO}_{2}$ (open blue circles). All data correspond to the case of frozen $U=0$ structures and CO suppressed in $\mathrm{Li}_{1 / 2} \mathrm{CoO}_{2} \ldots \ldots \ldots \ldots \ldots$

2.7 Filling factor $\mu(1-\mu)$ as a function of $x$ for different $U$ in $\mathrm{Li}_{x} \mathrm{CoO}_{2}$ for (a) frozen $U=0$ structures and CO suppressed, (b) frozen $U=0$ structures and CO allowed, and (c) relaxed structures. Panels (d)-(f) show the corresponding plots for the ordering factor $\sigma^{2}$. The two lines per $U$ in some plots correspond to the two distinct $\mathrm{Co}$ sites in $\mathrm{Li}_{1 / 2} \mathrm{CoO}_{2}$. The line thickness increases for increasing values of $U \ldots \ldots \ldots \ldots \ldots$ 
2.8 (a) Total $\mathrm{Li}_{1 / 2} \mathrm{CoO}_{2}$ formation energy (open black circles) and its DFT (filled red squares) and $U$-dc (filled blue circles) components as a function of $U$ (b) $\mathrm{Li}_{1 / 2} \mathrm{CoO}_{2} U$-dc (filled blue circles) formation energy component and its orbital filling (open red circles) and orbital ordering (open black squares) components as a function of $U$ (c) Number of $d$ electrons per Co as a function of $U$ for $\mathrm{CoO}_{2}$ (filled black circles), $\mathrm{Li}_{1 / 2} \mathrm{CoO}_{2}$ (open red squares), and $\mathrm{LiCoO}_{2}$ (open blue circles). All data correspond to the case of frozen $U=0$ structures and $\mathrm{CO}$ allowed and the two lines in panel (c) for $\mathrm{Li}_{1 / 2} \mathrm{CoO}_{2}$ correspond to the two distinct Co sites. . . . . . . . . . . . . . . . . . . .

2.9 (a) Total $\mathrm{Li}_{1 / 2} \mathrm{CoO}_{2}$ formation energy (open black circles) and its DFT (filled red squares) and $U$-dc (filled blue circles) components as a function of $U$ (b) $\mathrm{Li}_{1 / 2} \mathrm{CoO}_{2} U$-dc (filled blue circles) formation energy component and its orbital filling (open red circles) and orbital ordering (open black squares) components as a function of $U$ (c) Number of $d$ electrons per Co as a function of $U$ for $\mathrm{CoO}_{2}$ (filled black circles), $\mathrm{Li}_{1 / 2} \mathrm{CoO}_{2}$ (open red squares), and $\mathrm{LiCoO}_{2}$ (open blue circles). All data correspond to the case of fully relaxed structures and the two lines in panel (c) for $\mathrm{Li}_{1 / 2} \mathrm{CoO}_{2}$ correspond to the two distinct $\mathrm{Co}$

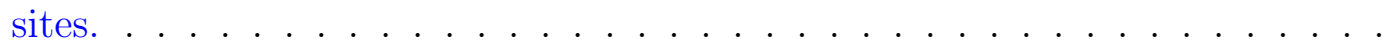

2.10 Electronic band gap as a function of $U$ for (a) $\mathrm{FePO}_{4}$, (b) $\mathrm{Li}_{1 / 4} \mathrm{FePO}_{4}$, (c) $\mathrm{Li}_{1 / 2} \mathrm{FePO}_{4}$, (d) $\mathrm{Li}_{3 / 4} \mathrm{FePO}_{4}$, and (e) $\mathrm{LiFePO}_{4}$ with relaxations (filled red squares) and with frozen linearly interpolated experimental structures (open black circles). The corresponding plots of total magnetization and Fe magnetic moment magnitudes are shown in panels $(\mathrm{f})-(\mathrm{j})$ and $(\mathrm{k})-(\mathrm{o})$, respectively. For panels (k)-(o), the multiple symbols per $U$ correspond to the 4 distinct Fe sites. 55 
2.11 Total $\mathrm{Li}_{x} \mathrm{FePO}_{4}$ formation energy (open black circles) and its DFT (filled red squares) and $U$-dc (filled blue circles) components as a function of $U$ for (a) $x=1 / 4$, (b), $1 / 2$, and (c) $x=3 / 4$. (d)-(f) show the corresponding plots of $\mathrm{Li}_{x} \mathrm{FePO}_{4} U-\mathrm{dc}$ (filled blue circles) formation energy component and its orbital filling (open red circles) and orbital ordering (open black squares) components as a function of $U$. (g)-(i) show the corresponding plots of number of $d$ electrons per $\mathrm{Fe}$ as a function of $U$ for $\mathrm{FePO}_{4}$ (black line), $\mathrm{Li}_{x} \mathrm{FePO}_{4}$ (open red squares), and $\mathrm{LiFePO}_{4}$ (blue line). All data correspond to the case of frozen linearly interpolated experimental structures. . . . . . . . . . . 57

2.12 Filling factor $\mu(1-\mu)$ as a function of $x$ averaged over Fe sites for different $U$ in $\mathrm{Li}_{x} \mathrm{FePO}_{4}$ for (a) linearly interpolated experimental structures and (b) relaxed structures. Panels (c) and (d) show the corresponding plots for the ordering factor $\sigma^{2}$. The line thickness increases for increasing values of $U$. . .

2.13 Total $\mathrm{Li}_{x} \mathrm{FePO}_{4}$ formation energy (open black circles) and its DFT (filled red squares) and $U$-dc (filled blue circles) components as a function of $U$ for (a) $x=1 / 4$, (b), $1 / 2$, and (c) $x=3 / 4$. (d)-(f) show the corresponding plots of $\mathrm{Li}_{x} \mathrm{FePO}_{4} U-\mathrm{dc}$ (filled blue circles) formation energy component and its orbital filling (open red circles) and orbital ordering (open black squares) components as a function of $U$. (g)-(i) show the corresponding plots of number of $d$ electrons per $\mathrm{Fe}$ as a function of $U$ for $\mathrm{FePO}_{4}$ (black line), $\mathrm{Li}_{x} \mathrm{FePO}_{4}$ (open red squares), and $\mathrm{LiFePO}_{4}$ (blue line). All data correspond to the case of relaxed structures. . . . . . . . . . . . . . . . . . 
2.14 Average intercalation voltage of (a) $\mathrm{Li}_{x} \mathrm{CoO}_{2}$ and (b) $\mathrm{Li}_{x} \mathrm{FePO}_{4}$ as a function of $U$ shown using relaxed structures and frozen $U=0$ relaxed structures. For $\mathrm{Li}_{x} \mathrm{FePO}_{4}$ the results using the experimental structures are also shown. Green lines indicate the range of the average voltage measured from experiment, and grey lines indicate $U$ values of the endmembers computed via the linear response method [Zhou et al. (2004a)]. . . . . . . . . . . . . . . .

2.15 (a) Order-disorder transition temperature, (b) Co magnetic moments, (c) Co $\mathrm{N}_{d}$, and (d) band gap for $\mathrm{Li}_{1 / 2} \mathrm{CoO}_{2}$ as a function of $U$ using the frozen $U=0$ $\mathrm{eV}$ structures. The disordered phase is modeled by the optimal 42 -ion special quasirandom structure. In panel (a) the horizontal red line indicates the experimental order-disorder transition temperature from Ref. 123. For the other panels black lines (red squares) correspond to results from the ordered (disordered) structure. . . . . . . . . . . . . . . . . .

2.16 Order-disorder transition temperature and Co magnetic moment, Co $N_{d}$, and band gap for ordered and disordered structures as a function of $U$. For each of the four SQS cells considered, the results without (with) structure relaxations are shown to the left (right). For the Co magnetic moment, Co $N_{d}$, and band gap, black lines and filled circles correspond to the ordered structure and red squares correspond to the disordered structure. The figure of merit (f.o.m.) for each SQS structure is indicated. . . . . . . . . . . . . . . . . 71

3.1 Density of states for DFT (black solid lines) and using the Wannier basis (dashed red lines) for (a) band insulating $\mathrm{LiCoO}_{2}$ and (b) metallic $\mathrm{CoO}_{2}$. (c) crystal structure of LCO with O3 layer stacking with all the Li shown $(x=1)$. The large green, medium blue, and small red spheres represent ionic positions of $\mathrm{Li}, \mathrm{Co}$, and $\mathrm{O}$, respectively. The image of the crystal structure is generated using vesta $[$ Momma and Izumi $(2011)] \ldots \ldots$. . . . . . . . . . 
3.2 Total energy of $\mathrm{CoO}_{2}$ (upper part) and $\mathrm{LiCoO}_{2}$ (lower part) as a function of $U$ for several methodologies including DFT, DFT $+U$, and DFT+DMFT. The dashed orange lines indicate the computed values of $U$ for $\mathrm{LiCoO}_{2}$ (lower value) and $\mathrm{CoO}_{2}$ (higher value) within the linear response approach. . . . . .

3.3 Imaginary part of the DMFT self-energy on the imaginary frequency axis for (a) $\mathrm{CoO}_{2}$ and (b) $\mathrm{LiCoO}_{2}$ with density-density interactions for different values of $U$. Solid, dashed, and dotted lines correspond to the $E_{g}^{\prime}, A_{1 g}$, and $E_{g}$ orbitals. (c) and (d) show the corresponding real parts referenced to the chemical potential. . . . . . . . . . . . . . . . . .

3.4 Imaginary part of the DMFT self-energy on the imaginary frequency axis for (a) $\mathrm{CoO}_{2}$ and (b) $\mathrm{LiCoO}_{2}$ including off-diagonal $J$ within the $E_{g}$ manifold for different values of $U$. Solid, dashed, and dotted lines correspond to the $E_{g}^{\prime}$, $A_{1 g}$, and $E_{g}$ orbitals. (c) and (d) show the corresponding real parts referenced to the chemical potential. . . . . . . . . . . . . . .

3.5 Quasiparticle weight $Z$ as a function of $U$ for (a) $E_{g}^{\prime}$, (b) $A_{1 g}$, and (c) $E_{g}$ orbitals. . . . . . . . . . . . . . . . . . .

3.6 Probability of Co atomic states with number of electrons $N$ and spin projection $S_{z}$ for $\left(\right.$ a) $\mathrm{CoO}_{2}$ and (b) $\mathrm{LiCoO}_{2} \ldots \ldots \ldots \ldots$

3.7 $N_{d}$ versus $U$ for (a) $\mathrm{LiCoO}_{2}$ and (b) $\mathrm{CoO}_{2}$ for all the methodologies employed in this study. The dashed orange lines indicate the computed values of $U$ for $\mathrm{LiCoO}_{2}$ (lower value) and $\mathrm{CoO}_{2}$ (higher value) within the linear response

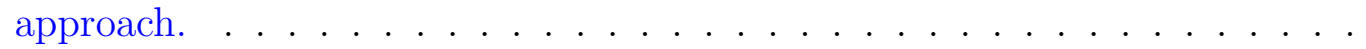

3.8 Total $T_{2 g}$ occupancy versus $U$ for (a) $\mathrm{LiCoO}_{2}$ and (b) $\mathrm{CoO}_{2}$ for all the methodologies employed in this study. (c) and (d) show the corresponding $E_{g}$ plots. 
3.9 Computed intercalation voltage of $\mathrm{LCO}$ via $\mathrm{DFT}, \mathrm{DFT}+U$, and $\mathrm{DFT}+\mathrm{DMFT}$ as a function of $U$. The dashed orange lines indicate the computed values of $U$ for $\mathrm{LiCoO}_{2}$ (lower value) and $\mathrm{CoO}_{2}$ (higher value) within the linear response approach. The dotted black lines indicate the expected range of the experimental result $[$ Amatucci et al. (1996)] . . . . . . . . . . . . . . .

4.1 Side view of crystal structures of trigonal prismatic and octahedral monolayer $\mathrm{VS}_{2}$ and schematic V $3 d$ orbital fillings from crystal field theory. The red and yellow spheres represent ionic positions of $\mathrm{V}$ and $\mathrm{S}$, respectively. . . . . . .

4.2 Orthographic projection along the out-of-plane axis of the (a) FM $U=0$ $q=2 / 3 K$ OCT and (b) FM $U=3.8 \mathrm{eV} q=3 / 5 K$ TP relaxed structures. Vanadium (sulfur) ions are indicated by red (yellow) spheres and the thick black lines show the shortest $\mathrm{V}-\mathrm{S}$ bonds. The unit cell is indicated by thin black lines. . . . . . . . . . . . . . . . . . . . . . .

4.3 NSP electronic band structure and total (solid black line), $d$ (solid red line), and $d_{3 z^{2}-r^{2}}$ (dashed blue line) density of states for TP $\mathrm{VS}_{2}$ within DFT. The black dotted line indicates the Fermi energy and the shaded areas illustrate the gaps around the isolated low-energy band. The $k$-point labels $\Gamma, M$, and $K$ correspond to the center, edge midpoint, and corner of the Brillouin zone, respectively. . . . . . . . . . . . . . . . . . . .

4.4 (a) Density of states and (b) Fermi surface for NSP TP VS 2 . The thick black lines correspond to DFT, while the thick red (thin blue) lines indicate tight binding results with (without) NN V-V hopping matrix elements. The dotted lines show the irreducible Brillouin zone. Corresponding plots for OCT $\mathrm{VS}_{2}$ are shown in panels $(\mathrm{c})$ and $(\mathrm{d}) \ldots \ldots \ldots$. . . . . . . . . . . . 107

4.5 Energy level diagram for TP (left, in red) and OCT (right, in blue) $\mathrm{VS}_{2}$ within DFT. The energy of the FM TP state is used as a reference energy. . . . . . 110 
4.6 Phonon dispersion relation and total (black) and V-projected (red) phonon density of states for FM TP $\mathrm{VS}_{2}$ within DFT. The band labels identify the mode character near the $\Gamma$ point. $\mathrm{z}, \mathrm{T}, \mathrm{L}, \mathrm{A}$ and $\mathrm{O}$ refer to out-of-plane, transverse, longitudinal, acoustic, and optical branches, respectively. . . . . . 112

4.7 (a) $\mathrm{V}-\mathrm{V}$ and (b) $\mathrm{V}-\mathrm{S}$ distances for $\mathrm{OCT} \mathrm{VS}_{2}$ in the pristine $\mathrm{FM}$ phase, $q=$ $2 / 3 K$ FM CDW phase, and $q=2 / 3 K$ AFM CDW phase as a function of $U$. The two green dashed lines for the low-temperature experiment of Sun et al. in panel (a) correspond to the two measured V-V distances. For comparison, the $U=0$ value for the pristine FM phase is also shown within the local density approximation (LDA) . . . . . . . . . . . . . . .

4.8 Electronic density of states for $\mathrm{FM} \mathrm{VS}_{2}$ in the (a) $\mathrm{TP}$ and (b) OCT phases for different values of $U$. The dotted black line indicates the Fermi level. . .

4.9 Phonon dispersion relation for FM TP $\mathrm{VS}_{2}$ for $U=3.0 \mathrm{eV}$ (thin solid black lines), $U=3.2 \mathrm{eV}$ (dashed thin blue lines), and $U=3.4 \mathrm{eV}$ (thick solid red line). . . . . . . . . . . . . . . . . . .

4.10 (a) S-V-S bond angle, (b) V-S bond-length, (c) out-of-plane S-S distance, and (d) electronic band gap as a function of $U$ for FM TP $\mathrm{VS}_{2}$. The density matrix difference for $U=4 \mathrm{eV}$ (ground state minus metastable state) for spin-up (left) and spin-down (right) electrons is displayed in panel (e). The matrix rows (columns) correspond to $d_{x y}, d_{y z} d_{3 z^{2}-r^{2}}, d_{x z}$, and $d_{x^{2}-y^{2}}$ states from top to bottom (left to right). . . . . . . . . . . .

4.11 Total energy of NSP TP (black dashed line and open circles), NSP OCT (red dashed line and open squares), and FM OCT (red solid line and filled squares) states referenced to the FM TP (black solid line and filled circles) state energy as a function of $U$. The FM CDW state for the TP phase (purple solid triangles) and OCT phase (green upside-down triangles) are a small perturbation on the energetics. . . . . . . . . . . . . . . . 
4.12 (a) $\mathrm{DFT}+U$ total energy of FM OCT phase minus that of FM TP phase (red squares) and decomposition into DFT (black circles), filling (green triangles), and ordering (blue diamonds) contributions as a function of $U$. (b) $\mu(1-$ $\mu$ ) (green) and (c) $\sigma^{2}$ (blue) as a function of $U$. Solid (dashed) lines with (without) symbols correspond to the TP (OCT) phase in panels (b) and (c).

125 


\section{Acknowledgments}

First I would like to thank my advisor, Prof. Chris Marianetti, for the immense amount of knowledge, guidance, and wisdom he shared with me during my years at Columbia. I have truly enjoyed learning from and working with him on many interesting materials and fascinating problems in physics. I have no doubt that the lessons I have learned from him will be useful in my career and life.

I am very grateful to my thesis committee members Profs. Simon Billinge, Kyle Mandli, David Reichman, and Yuan Yang for their feedback and support. Additional thanks to Profs. Philip Kim, Aron Pinczuk, and Latha Venkataraman for administering my previous doctoral examinations.

I was very lucky to interact with wonderful colleagues in our group and more broadly at Columbia. Thanks to the postdocs Hyowon Park, Yue Chen, Hanghui Chen, Pierre Darancet, Emanuel Lazar, Dalal Kanan, Jia Chen, and Alex Taekyung Lee for your willingness to lend an ear whenever I was stuck on a problem. I enjoyed many discussions with Xinyuan Ai, Chanul Kim, Mordechai Kornbluth, Zhengqian Chen, Nader Zaki, Se Young Park, Hung Dang, Ryan Cooper, and Runzhi Wang. My Ph.D. experience would have been drastically inferior without Dov Rhodes, Gali Galwaduge, Austin Cheng, Olgun Adak, Diego Scarabelli, Max Terban, Vernon Wong, Tony Clark, Hande Öztürk, Timothy Liu, Garrett Rogren, Kevin Jeng, Brian Capozzi, Haixing Li, Soham Banerjee, and Jeffrey Taylor. 
Thanks to Montse Fernandez-Pinkley, Dina Amin, and Christina Rohm for helping me navigate the department and Columbia. I am especially grateful to the Department of Energy Computational Science Graduate Fellowship (Grant No. DE-FG02-97ER25308) for funding and support, and I appreciate the plentiful assistance of Jeana Gingery and Lindsey Eilts. As part of this program, I had the pleasure of spending a summer at Brookhaven National Laboratory and began a fruitful collaboration with Yan Li and Shangmin Xiong. I am also thankful for additional support from the National Science Foundation (CMMI-0927891 and DMR-1420634) and the Department of Energy (DE-AC02-05CH11231 and DE-AC02-98CH10886).

To Zach, Craig, Cheskie, Deena, Jacob, Rachael, Fatema, Tammy, Samara, Guillaume, Mira, Avner, Theo, Sarah, Julia, Asher, Ariela, Raphael, Allen, Dani, Geoffrey, Mayla, Diane, Josh, Ben and others: thank you for your friendship, the good times, and the delicious food.

Finally, I want to give my warmest thank you to my parents, to my brother Jason, to all of my family, and to Kay for their love, patience, and support. 


\section{To my grandfather Harold Wenig}




\section{Chapter 1}

\section{Introduction}

\subsection{The many-body problem in electronic structure}

The underlying equation describing the properties of a molecule or a solid are known from quantum mechanics. Ignoring relativistic effects, which are typically only relevant for compounds containing heavy elements, the Hamiltonian that governs a material is

$$
\hat{H}_{\mathrm{el}-\mathrm{ion}}=-\sum_{\alpha} \frac{\hbar^{2}}{2 M_{\alpha}} \nabla_{\alpha}^{2}-\sum_{i} \frac{\hbar^{2}}{2 m} \nabla_{i}^{2}+\sum_{\alpha \neq \beta} \frac{Z_{\alpha} Z_{\beta} e^{2}}{\left|\mathbf{R}_{\alpha}-\mathbf{R}_{\beta}\right|}+\sum_{i \neq j} \frac{e^{2}}{\left|\mathbf{r}_{i}-\mathbf{r}_{j}\right|}-\sum_{i, \alpha} \frac{Z_{\alpha} e^{2}}{\left|\mathbf{r}_{i}-\mathbf{R}_{\alpha}\right|}
$$

where $\mathbf{R}_{\alpha}$ is the position of nucleus $\alpha$ with mass $M_{\alpha}$ and charge $Z_{\alpha} e$ and $\mathbf{r}_{i}$ is the position of electron $i$ with mass $m$ and charge $-e$. The terms correspond to the kinetic energy of the nuclei and electrons, the nuclei-nuclei and electron-electron Coulomb interactions, and the electron-nuclei Coulomb interaction. Since their mass is much larger than the electrons, the nuclei can be treated separately (the Born-Oppenheimer approximation). In this case, treating the nuclei classically, the nuclear positions $\left\{\mathbf{R}_{\alpha}\right\}$ enter as parameters in the total energy expression

$$
E\left(\left\{\mathbf{R}_{\alpha}\right\}\right)=\sum_{\alpha} \frac{\left|\mathbf{p}_{\alpha}\right|^{2}}{2 M_{\alpha}}+\sum_{\alpha \neq \beta} \frac{Z_{\alpha} Z_{\beta} e^{2}}{\left|\mathbf{R}_{\alpha}-\mathbf{R}_{\beta}\right|}+E_{\text {elec }}\left(\left\{\mathbf{R}_{\alpha}\right\}\right)
$$

where $\mathbf{p}_{\alpha}$ and $\mathbf{R}_{\alpha}$ are the classical momentum and position vectors (rather than operators). The electronic energy $E_{\text {elec }}$ is determined by solving to the Schrödinger equation 
$\hat{H}_{\text {elec }} \Psi\left(\left\{\mathbf{r}_{i}\right\}\right)=E_{\text {elec }} \Psi\left(\left\{\mathbf{r}_{i}\right\}\right)$, with the electronic Hamiltonian given by

$$
\hat{H}_{\text {elec }}=-\sum_{i} \frac{\hbar^{2}}{2 m} \nabla_{i}^{2}+\sum_{i \neq j} \frac{e^{2}}{\left|\mathbf{r}_{i}-\mathbf{r}_{j}\right|}-\sum_{i, \alpha} \frac{Z_{\alpha} e^{2}}{\left|\mathbf{r}_{i}-\mathbf{R}_{\alpha}\right|}
$$

Although this equation can be easily written down, it is hopelessly difficult to solve for any system with more than a few electrons. The fundamental difficulty is the fact that the dimensionality of the problem grows extremely rapidly with the size of the system since the fundamental quantity, the many-body wavefunction $\Psi\left(\left\{\mathbf{r}_{i}\right\}\right)$, depends on the positions of all the $N_{\text {elec }}$ electrons. In other words, it is a $3 N_{\text {elec }}$-dimensional function (the factor of 3 coming from the number of spatial dimensions). The size of the wavefunction $\Psi$ increases exponentially with $N_{\text {elec }}$, and therefore the time to solve the equation increases exponentially or faster. To illustrate the basic problem, suppose there is a single-electron $\left(N_{\text {elec }}=1\right)$ problem that can be solved numerically on a modern computer in one second. Table 1.1 illustrates how the time to solve increases extremely rapidly with $N_{\text {elec }}$ : once we are interested in even a 20-electron problem it will take over five years to get the answer.

\begin{tabular}{lr}
\hline \hline$N_{\text {elec }}$ & time to solve \\
\hline 1 & 1 second \\
2 & 2.7 seconds \\
10 & 2.3 hours \\
20 & 5.7 years \\
100 & $3.1 \times 10^{35}$ years \\
\hline
\end{tabular}

Table 1.1: Exponential scaling of the time to solve the many-electron Schrödinger equation, assuming a single-electron problem takes 1 second to solve numerically and the scaling prefactor is unity. The time to solve a 100 -electron problem $\left(10^{35}\right.$ years) is astronomical.

Paul Dirac best described this conundrum, known as the "many-body problem," in his famous quote [Dirac (1929)]: "The underlying physical laws necessary for the mathematical theory of a large part of physics and the whole of chemistry are thus completely known, and the difficulty is only that the exact application of these laws leads to equations much too complicated to be soluble." 


\subsection{Density functional theory}

There are generally two classes of approaches aimed at overcoming the many-body problem in electronic structure. In the first, from the chemistry community, one continues to work with the many-body wavefunction $\Psi$ but makes approximations to its form. Some examples are Møller-Plesset perturbation theory, configuration interaction, and coupled cluster. While such quantum chemistry approaches are extremely valuable, they are often less utilized outside the study of relatively small molecules since the scaling of the problem with $N_{\text {elec }}$ is still

usually prohibitive (e.g. $\mathcal{O}\left(N_{\text {elec }}^{5-7}\right)$ ). However, there is a widely-used alternative approach from the physics community called density functional theory (DFT). The idea is to avoid $\Psi$ entirely as its size is massive and it contains much more information than we likely need. In DFT, the electron density $\rho(\mathbf{r})$ is taken as the fundamental variable instead of $\Psi$.

There are two fundamental underpinnings to DFT. The first, shown by Pierre Hohenberg and Walter Kohn in 1964, is that the total energy of any $N_{\text {elec }}$-electron system is a unique functional of $\rho(\mathbf{r})$ that is minimized for the ground state [Hohenberg and Kohn (1964)]. This surprising result known as the Hohenberg-Kohn theorem demonstrates that, if we restrict our attention to the ground state, in principle one can circumvent dealing with the complicated many-body wavefunction $\Psi$ and instead deal with the much simpler function $\rho(\mathbf{r})$.

The second component comes from Walter Kohn and Lu Sham, who a year later showed that one can construct the exact electron density as

$$
\rho(\mathbf{r})=\sum_{i=1}^{N_{\text {elec }}}\left|\psi_{i}(\mathbf{r})\right|^{2},
$$

where $\left\{\psi_{i}(\mathbf{r})\right\}$ are auxiliary single-electron wavefunctions (orbitals) corresponding to noninteracting electrons in a special effective potential [Kohn and Sham (1965)]. This "Kohn- 
Sham potential" takes the form

$$
V_{K S}(\mathbf{r})=-\sum_{\alpha} \frac{Z_{\alpha} e^{2}}{\left|\mathbf{r}-\mathbf{R}_{\alpha}\right|}+\frac{e^{2}}{2} \int \frac{\rho\left(\mathbf{r}^{\prime}\right)}{\left|\mathbf{r}-\mathbf{r}^{\prime}\right|} \mathrm{d} \mathbf{r}^{\prime}+V_{x c}(\mathbf{r})
$$

Here the first term is the electron-nucleus interaction, the second term is the average (meanfield or Hartree) Coulomb energy, and the exchange-correlation potential $V_{x c}$ contains in it all the effects not taken into account by the single-particle kinetic energy $\left(\frac{-\hbar^{2}}{2 m} \nabla^{2}\right)$ or the other two terms in $V_{K S}$. In this context, correlation generally refers to the interactions beyond the Hartree term and exchange refers to the effect of the Pauli exclusion principle. Formally, $V_{x c}$ is the functional derivative of an exchange-correlation energy $E_{x c}[\rho]$ with respect to the density $\rho$ :

$$
V_{x c}(\mathbf{r})=\frac{\delta E_{x c}[\rho]}{\delta \rho(\mathbf{r})}
$$

The fundamental idea of DFT, as shown conceptually in Figure 1.1, is that we have mapped a very complicated $N_{\text {elec}}$-electron problem with all the interactions onto a much simpler 1-electron (non-interacting) problem with an effective potential.

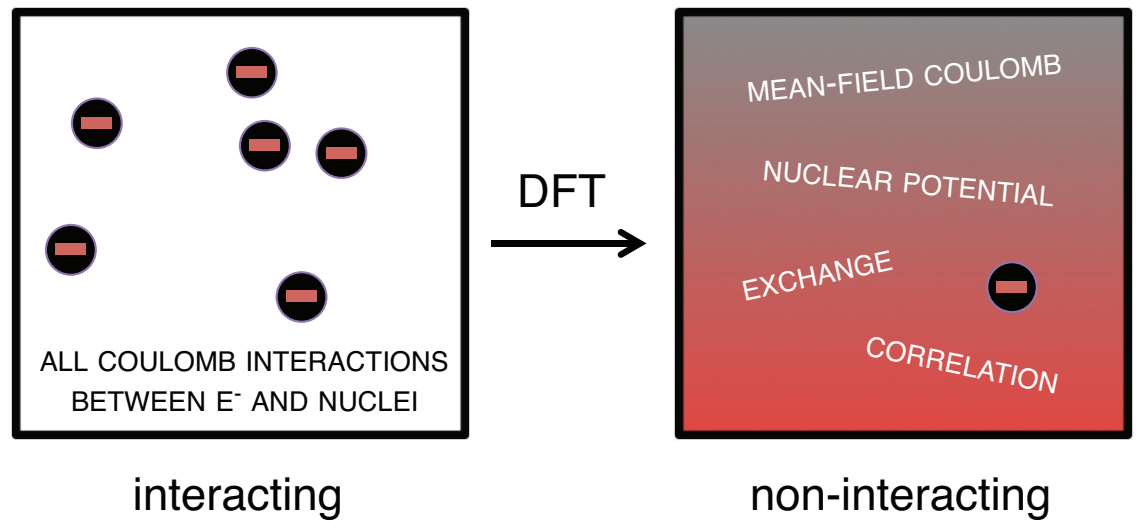

Figure 1.1: DFT maps an interacting $N_{\text {elec }}$-electron problem with the full interactions onto an auxiliary 1-electron problem with an effective Kohn-Sham potential containing contributions from the electron-nucleus interaction, mean-field Coulomb interaction, and the exchange-correlation potential. In this schematic, the black circles correspond to electrons and the gradient on the right side represents the fictitious Kohn-Sham potential. 
The beauty of this theory is that we have mapped an intractable $3 N$-dimensional problem into a much simpler 3-dimensional problem. The "catch" here is that while we know the exchange-correlation potential exists, we do not know its exact form. In practice we must make approximations to this part of the Kohn-Sham potential. The first approximation to $E_{x c}$, proposed by Kohn and Sham in their paper and still often used today, is called the local density approximation (LDA). It assumes that at a particular point in space $E_{x c}(\mathbf{r})$ is equal to $E_{x c}$ of a model system called the homogeneous electron gas of the same fixed density, which has been exactly numerically computed using quantum Monte Carlo calculations. Another widely-used approximation is the generalized gradient approximation (GGA) (e.g. that of Perdew, Burke, and Ernzerhof [Perdew et al. (1996)]), which goes a step beyond the LDA by also including dependence on the gradient of the density $\nabla \rho$. Note additionally that this is a nonlinear partial differential equation since the single-particle wavefunctions $\psi$ enter into the Kohn-Sham potential via $\rho$, so the equations must be solved iteratively. DFT is referred to as a "first-principles" or sometimes "ab initio" theory since usually there is no empiricism in the approximations of the Kohn-Sham potential.

Walter Kohn won half of the Nobel Prize in Chemistry in 1998 for DFT. It has been enormously successful in physics, chemistry, and materials science and is applied to materials with elements across the periodic table. As such, DFT is considered to be the "standard theory" of materials science. The fundamental quantities it provides are the total energy and electron density. From derivatives of the total energy, it can tell us forces on the nuclei as well as atomic vibrations (phonons). Furthermore, by interpreting the auxiliary Kohn-Sham states as the actual wavefunctions much additional information can be predicted such as the band structure and density of states. 


\section{$1.3 \quad$ Strongly correlated materials}

Correlated materials are systems in which the Coulomb interactions in an open $d$ or $f$ electron shell are especially strong. Examples are transition metal oxides, actinides, heavy fermion materials, and organic charge transfer salts. These materials can exhibit magnetism, metal-insulator transitions, superconductivity, and charge density waves. Correlated materials host some of the most fascinating phenomena such as high-temperature superconductivity and colossal magnetoresistance. As such, it would be highly desirable to properly describe correlated materials from theory.

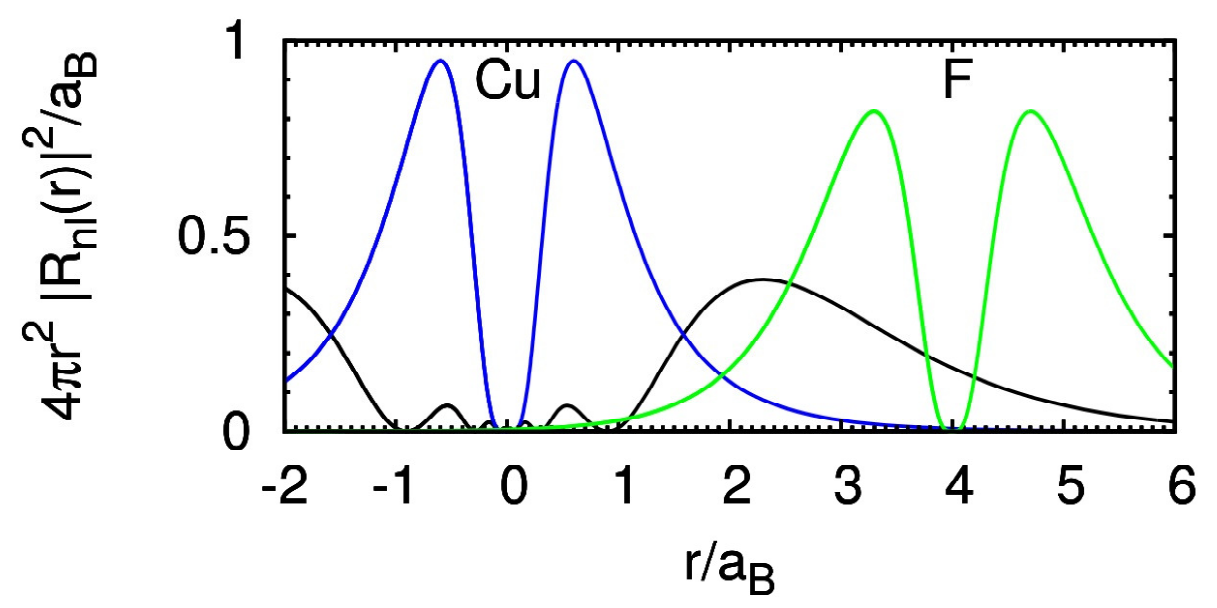

Figure 1.2: Radial probability distribution for $\mathrm{Cu} 4 s$ (black), $\mathrm{Cu} 3 d$ (blue), and $\mathrm{F} 2 p$ based on LDA calculations of single atoms. The $\mathrm{F} 2 p$ curve is centered at $r=4 a_{B}$, where $a_{B}$ is the Bohr radius. $\mathrm{Cu}$ and $\mathrm{F}$ are in in the [Ar] $3 d^{10} 4 s^{1}$ and [He] $2 s^{2} 2 p^{5}$ configurations, respectively. Figure taken from Pavarini et al. (2011).

Figure 1.2 illustrates the much more delocalized nature of $s$ and $p$ electrons using the example of isolated $\mathrm{Cu}$ and $\mathrm{F}$ atoms separated by 4 times the Bohr radius. Since $d$ and $f$ electrons are much more localized, the Coulomb repulsion between them is especially large. In correlated materials this leads to significant many-body effects in open $d$ or $f$ shells. Although it is an exact many-body theory for the ground-state energy and density, in practice DFT fails to properly capture this strong Coulomb interaction. Furthermore, the 
interpretation of the Kohn-Sham states as approximate quasiparticle states as is often done in DFT (though not completely justified) typically fails very severely for correlated materials unlike for noncorrelated materials.

The most important failure of DFT in this context is the description of the Mott insulator [Imada et al. (1998)]. This class of materials has an odd number of electrons, so band theory (and typically DFT) predicts they are metallic. In reality they are strongly insulating even in the paramagnetic phase above the magnetic ordering temperature since the strong Coulomb interaction localizes the electrons. A couple of classic examples are $\mathrm{NiO}$ and $\mathrm{LaTiO}_{3}$.

\subsubsection{Hubbard model}

The minimal model Hamiltonian believed to describe the physics of strongly correlated systems is the famous Hubbard model [Kanamori (1963); Hubbard (1963); Gutzwiller (1963)], which for a single orbital is

$$
\hat{H}_{H u b b a r d}=-t \sum_{\langle i, j\rangle, \sigma} \hat{c}_{i \sigma}^{\dagger} \hat{c}_{j \sigma}+U \sum_{i} \hat{n}_{i \uparrow} \hat{n}_{i \downarrow}
$$

where $\hat{c}_{i \sigma}^{\dagger}\left(\hat{c}_{i \sigma}\right)$ is the creation (annihilation) operator in the second quantization picture for lattice site $i$ and spin $\sigma$ and $\hat{n}_{i \sigma}=\hat{c}_{i \sigma}^{\dagger} \hat{c}_{i \sigma}$ is the corresponding number operator. Here $t$ is the hopping parameter, which is proportional to overlap between the orbitals of nearest neighbor sites and gives the electronic bandwidth, and $U$ is the on-site Coulomb repulsion strength.

The Hubbard model shown schematically in Fig. 1.3 describes the competition between the hopping, which favors delocalized metallic states, and the tendency for electronic localization and insulating behavior due to $U$. In this model only the strongest contribution to the Coulomb interaction, that on a single lattice site, is retained. The $\langle i, j\rangle$ indicates we only include nearest-neighbor hopping as in simple tight binding models, though this is easy to generalize to longer-range hopping. Although the Hubbard model is very easy to write 


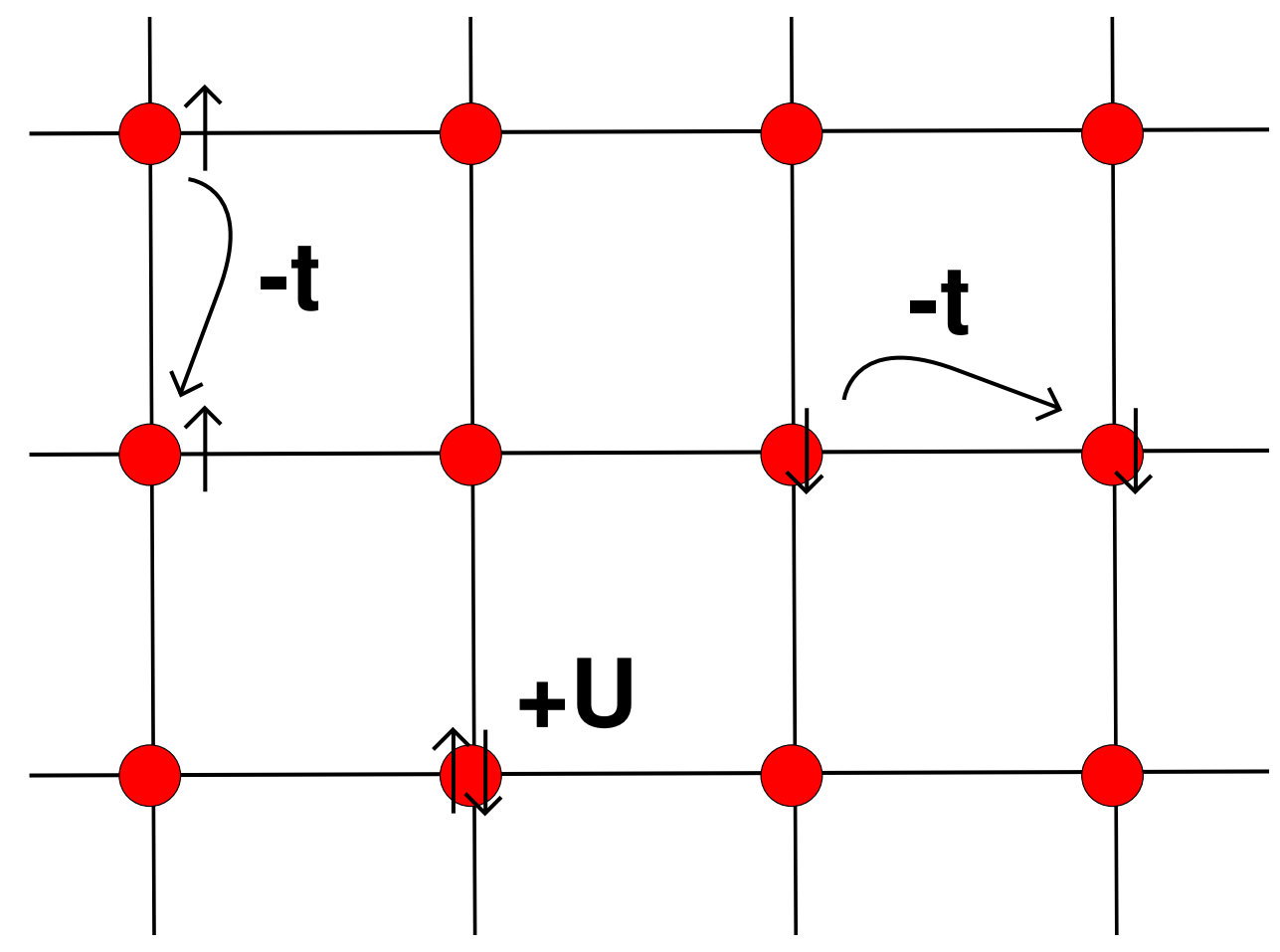

Figure 1.3: Schematic of the Hubbard model on a two-dimensional square lattice. Electron hopping between sites is favorable with energy $-t$. Double occupancy on a single site results in an energy penalty of $+U$. 
down, this is a many-body problem that is generally very difficult to solve. Exact solutions only exist in one and infinite dimensions. In the strongly correlated electron materials community, there has been a large amount of effort to solve this and other model Hamiltonians since an ab initio theory has been lacking.

\subsection{DFT plus Hubbard $U$}

In the DFT plus Hubbard $U(\mathrm{DFT}+U)$ method, DFT is augmented with an explicit though approximate treatment of the Coulomb interaction for the strongly correlated electronic degrees of freedom [Anisimov et al. (1991, 1993); Liechtenstein et al. (1995); Anisimov et al. (1997); Dudarev et al. (1998); Himmetoglu et al. (2014)]. This approach provides a means to correct some of the deficiencies of pure DFT for strongly correlated materials and is a useful and popular tool in electronic structure theory.

As summarized in Sec. 1.2, pure DFT is a theory in which the electron density $\rho(\mathbf{r})$, a three-dimensional function, is the fundamental variable. However, one is free to construct alternative theories based on other variables containing more information. The general idea is that variables other than the electron density can be better suited to capture the physics of correlated materials.

In $\mathrm{DFT}+U$ we construct a theory in which the occupancies of localized orbitals are primary variables in addition to $\rho(\mathbf{r})$. The underlying logic here is to isolate the part of the Hilbert space in which the correlations are particularly strong, for example the transition metal $d$ orbitals, and to provide an improved treatment of the interactions in this subspace. Typically this partitioning is done using Wannier functions [Marzari and Vanderbilt (1997)] or atomic-like orbitals $\left\{\left|\phi_{m}^{\tau}\right\rangle\right\}$ where $m$ labels the orbital and $\tau$ labels the atomic site.

While in DFT the total energy is a functional solely of $\rho(\mathbf{r})$, in DFT $+U$ a dependence 
on the occupancies of these correlated orbitals

$$
n_{m m^{\prime}}^{\tau \sigma}=\sum_{\mathbf{k}, v} f_{\mathbf{k} v \sigma}\left\langle\psi_{\mathbf{k} v \sigma} \mid \phi_{m^{\prime}}^{\tau}\right\rangle\left\langle\phi_{m}^{\tau} \mid \psi_{\mathbf{k} v \sigma}\right\rangle
$$

is also included. Here $\left|\psi_{\mathbf{k} v \sigma}\right\rangle$ is the Kohn-Sham state of crystal momentum $\mathbf{k}$, band $v$, and spin $\sigma$ and $f_{\mathbf{k} v \sigma}$ is the corresponding Fermi-Dirac occupation factor. This gives a modified total energy expression of

$$
E_{D F T+U}\left[\rho, n^{\tau \sigma}\right]=E_{D F T}[\rho]+E_{U}\left[n^{\tau \sigma}\right]-E_{d c}\left[n^{\tau \sigma}\right]
$$

where $E_{D F T}[\rho]$ is the total energy from DFT, $E_{U}\left[n^{\tau \sigma}\right]$ is the on-site Coulomb energy for the correlated orbitals, and $E_{d c}\left[n^{\tau \sigma}\right]$ is a double counting term whose aim is to subtract out the portion of $E_{U}\left[n^{\tau \sigma}\right]$ already captured by $E_{D F T}[\rho]$.

\subsubsection{Basic form}

In the spirit of a multi-orbital Hubbard model, we take our interaction Hamiltonian to be

$$
\begin{aligned}
\hat{H}_{U} & =U\left(\sum_{\tau, m} \hat{n}_{m}^{\tau \uparrow} \hat{n}_{m}^{\tau \downarrow}+\sum_{\tau, m \neq m^{\prime}} \hat{n}_{m}^{\tau \uparrow} \hat{n}_{m^{\prime}}^{\tau \downarrow}+\sum_{\tau, m>m^{\prime}, \sigma} \hat{n}_{m}^{\tau \sigma} \hat{n}_{m^{\prime}}^{\tau \sigma}\right) \\
& =U\left(\frac{1}{2} \sum_{\tau, m, \sigma \neq \sigma^{\prime}} \hat{n}_{m}^{\tau \sigma} \hat{n}_{m}^{\tau \sigma^{\prime}}+\frac{1}{2} \sum_{\tau, m \neq m^{\prime}, \sigma \neq \sigma^{\prime}} \hat{n}_{m}^{\tau \sigma} \hat{n}_{m^{\prime}}^{\tau \sigma^{\prime}}+\frac{1}{2} \sum_{\tau, m \neq m^{\prime}, \sigma} \hat{n}_{m}^{\tau \sigma} \hat{n}_{m^{\prime}}^{\tau \sigma}\right) \\
& =\frac{1}{2} U\left(\sum_{\tau, m, m^{\prime}, \sigma \neq \sigma^{\prime}} \hat{n}_{m}^{\tau \sigma} \hat{n}_{m^{\prime}}^{\tau \sigma^{\prime}}+\sum_{\tau, m \neq m^{\prime}, \sigma=\sigma^{\prime}} \hat{n}_{m}^{\tau \sigma} \hat{n}_{m^{\prime}}^{\tau \sigma^{\prime}}\right) \\
& =\frac{1}{2} U \sum_{\tau, m \sigma \neq m^{\prime} \sigma^{\prime}} \hat{n}_{m}^{\tau \sigma} \hat{n}_{m^{\prime}}^{\tau \sigma^{\prime}}
\end{aligned}
$$


Here for simplicity we are assuming there is a single correlated atomic species with on-site Coulomb repulsion $U$, though this can be easily generalized to more complex situations. The value of $U$ is often taken as an adjustable parameter, though there are approaches to compute it from first principles such as the linear response approach [Cococcioni and de Gironcoli (2005)]. The total interaction energy $E_{U}$ is equal to $\left\langle\hat{H}_{U}\right\rangle$. Using a HartreeFock approximation $\left\langle\hat{n}_{m}^{\tau \sigma} \hat{n}_{m^{\prime}}^{\tau \sigma^{\prime}}\right\rangle=\left\langle\hat{n}_{m}^{\tau \sigma}\right\rangle\left\langle\hat{n}_{m^{\prime}}^{\tau \sigma^{\prime}}\right\rangle$ we get

$$
E_{U}=\frac{1}{2} U \sum_{\tau, m \sigma \neq m^{\prime} \sigma^{\prime}} n_{m m}^{\tau \sigma} n_{m^{\prime} m^{\prime}}^{\tau \sigma^{\prime}}
$$

Note that the use of Hartree-Fock is the main approximation in this method. DFT $+U$ is an approximation to more advanced theories such as DFT plus dynamical mean-field theory (see Sec. 1.5) in which beyond-Hartree-Fock techniques are used to solve the correlation problem.

Since there is typically no unique way to determine the portion of $E_{U}$ already taken into account within DFT, in practice one must choose some form of the double counting energy $E_{d c}$. There are two forms popular in the literature. The first is called fully-localized-limit (FLL) double counting. The idea here is that in a very correlated material the electronic states of the correlated atom are very localized and atomic-like with occupancies of either zero or one. In this limit we compute $E_{d c}$ as

$$
\begin{aligned}
E_{d c}=\left.E_{U}\right|_{F L L} & =\left.\frac{1}{2} U \sum_{\tau, m \sigma \neq m^{\prime} \sigma^{\prime}} n_{m m}^{\tau \sigma} n_{m^{\prime} m^{\prime}}^{\tau \sigma^{\prime}}\right|_{F L L} \\
& =\left.\frac{1}{2} U\left(\sum_{\tau, m, \sigma, m^{\prime}, \sigma^{\prime}} n_{m m^{\tau}}^{\tau \sigma} n_{m^{\prime} m^{\prime}}^{\tau \sigma^{\prime}}-\sum_{\tau, m, \sigma} n_{m m}^{\tau \sigma} n_{m m}^{\tau \sigma}\right)\right|_{F L L} \\
& =\frac{1}{2} U \sum_{\tau}\left(N_{\tau}^{2}-N_{\tau}\right)=\frac{1}{2} U \sum_{\tau} N_{\tau}\left(N_{\tau}-1\right),
\end{aligned}
$$

where $N_{\tau}=\sum_{m, \sigma} n_{m m}^{\tau \sigma}$ is the total number of electrons in the correlated shell on site $\tau$. An 
alternative form for less correlated situations is around-mean-field (AMF) double counting, which supposes that within DFT each orbital on site $\tau$ should have an average occupancy $\left\langle n_{\tau}\right\rangle$.

$$
\begin{aligned}
E_{d c}=\left.E_{U}\right|_{A M F} & =\left.\frac{1}{2} U \sum_{\tau} \sum_{m \sigma \neq m^{\prime} \sigma^{\prime}} n_{m m^{\tau}}^{\tau \sigma} n_{m^{\prime} m^{\prime}}^{\tau \sigma^{\prime}}\right|_{A M F} \\
& =\frac{1}{2} U \sum_{\tau}\left\langle n_{\tau}\right\rangle^{2} \sum_{m \sigma \neq m^{\prime} \sigma^{\prime}} 1 \\
& =\frac{1}{2} U \sum_{\tau}\left\langle n_{\tau}\right\rangle^{2}\left(\sum_{m, \sigma, m^{\prime}, \sigma^{\prime}} 1-\sum_{m \sigma=m^{\prime} \sigma^{\prime}} 1\right) \\
& =\frac{1}{2} U \sum_{\tau}\left\langle n_{\tau}\right\rangle^{2}\left[\left(\sum_{m, \sigma} 1\right)^{2}-\left(\sum_{m, \sigma} 1\right)\right] \\
& =\frac{1}{2} U \sum_{\tau} N_{\tau}^{2}\left(1-\frac{1}{\sum_{m, \sigma} 1}\right) \\
& =\frac{1}{2} U \sum_{\tau} N_{\tau}\left(N_{\tau}-\left\langle n_{\tau}\right\rangle\right)
\end{aligned}
$$

From here on only the FLL double counting, which is the most popular form, is considered. This gives our overall energy functional as

$$
E_{D F T+U}\left[\rho, n^{\tau \sigma}\right]=E_{D F T}[\rho]+\frac{1}{2} U \sum_{\tau, m \sigma \neq m^{\prime} \sigma^{\prime}} n_{m m}^{\tau \sigma} n_{m^{\prime} m^{\prime}}^{\tau \sigma^{\prime}}-\frac{1}{2} U \sum_{\tau} N_{\tau}\left(N_{\tau}-1\right)
$$

Notice that only $E_{U}$ contains orbitally-dependent terms. The double counting term only depends on the trace of the density matrix, which makes sense since DFT is only concerned with the density. 
The $U$ and double counting terms can be written together in the following way:

$$
\begin{aligned}
E_{U}-E_{d c} & =\frac{1}{2} U \sum_{\tau}\left[N_{\tau}^{2}-\sum_{m, \sigma}\left(n_{m m}^{\tau \sigma}\right)^{2}\right]-\frac{1}{2} U \sum_{\tau}\left(N_{\tau}^{2}-N_{\tau}\right) \\
& =\frac{1}{2} U \sum_{\tau}\left[N_{\tau}-\sum_{m, \sigma}\left(n_{m m}^{\tau \sigma}\right)^{2}\right] \\
& =\frac{1}{2} U \sum_{\tau, m, \sigma} n_{m m}^{\tau \sigma}\left(1-n_{m m}^{\tau \sigma}\right)
\end{aligned}
$$

The form $n_{m m}^{\tau \sigma}\left(1-n_{m m}^{\tau \sigma}\right)$ shows that the correction to the total energy serves to penalize fractional occupancy of the correlated orbitals. In other words, this term penalizes deviations from idempotency (the condition of having eigenvalues of 0 or 1) of the density matrix.

In the plot of total energy versus number of electrons shown in Fig. 1.4, approximate DFT gives a smooth, roughly quadratic curve. However, for exact DFT it can be shown that one should have a piecewise continuous function with discontinuities in the derivative at points of integer number of electrons [Perdew et al. (1982)]. This lack of a derivative discontinuity is a well-known deficiency of approximate functionals like LDA and GGA that is partly responsible for the underestimation of electronic band gaps. Since the correction in DFT $+U$ has the form $n_{m m}^{\tau \sigma}\left(1-n_{m m}^{\tau \sigma}\right)$, one interpretation is that this helps to restore the missing derivative discontinuity. This is consistent with improved prediction of electronic band gaps for correlated materials within $\mathrm{DFT}+U$.

We now also need to determine how the additions to the total energy functional change 


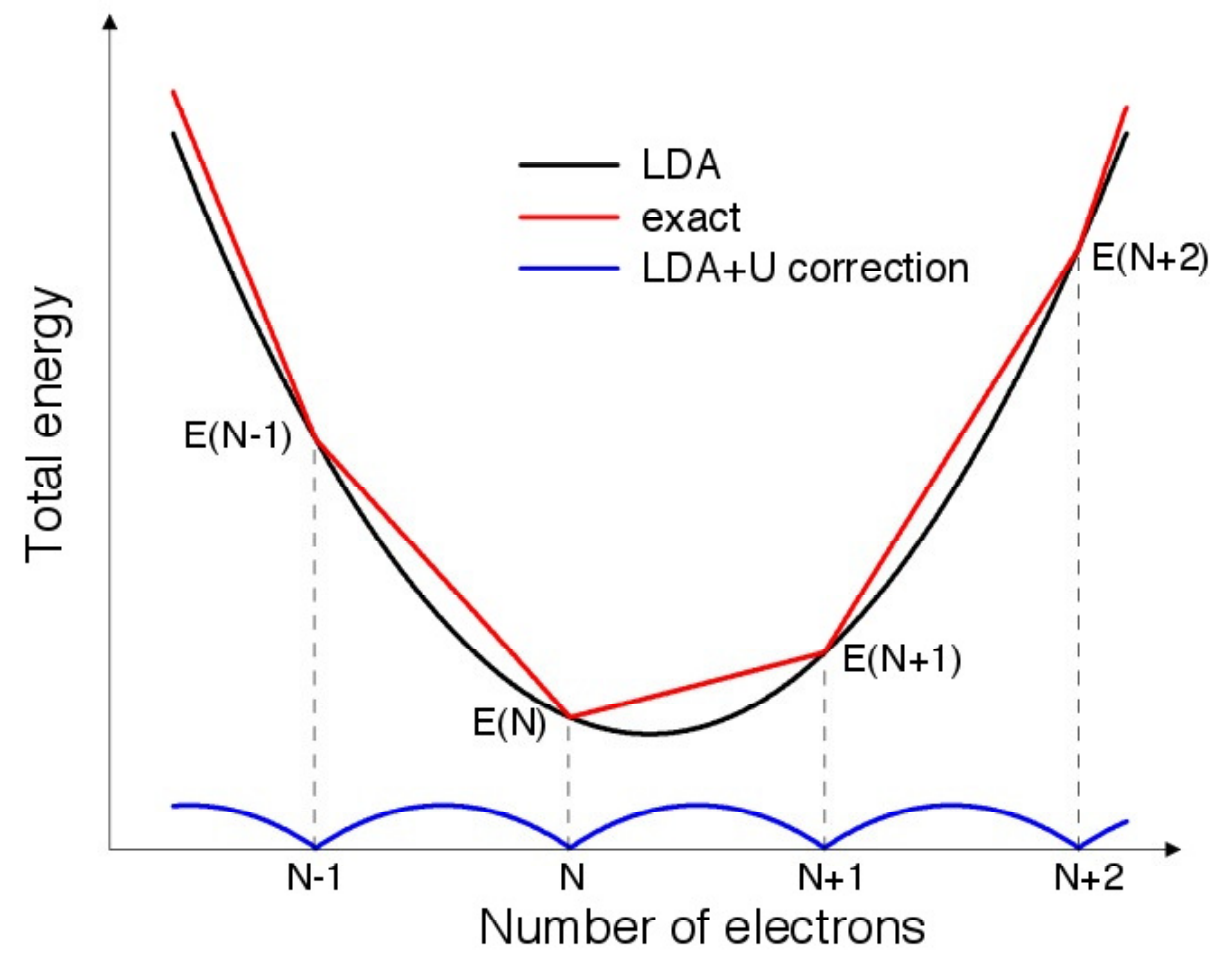

Figure 1.4: Schematic of total energy versus number of electrons $N$ for exact (red) and approximate (black) DFT. The correction to the total energy from the $\mathrm{DFT}+U$ method shown in blue can be viewed as a means to help restore the missing derivative discontinuity in approximate DFT. Figure taken from Cococcioni and de Gironcoli (2005). 
the effective single-particle potential in a calculation.

$$
\begin{aligned}
V_{U}=\frac{\partial E_{U}}{\partial n_{M M}^{T S}} & =\frac{1}{2} U \frac{\partial}{\partial n_{M M}^{T S}}\left(\sum_{\tau, m \sigma \neq m^{\prime} \sigma^{\prime}} n_{m m}^{\tau \sigma} n_{m^{\prime} m^{\prime}}^{\tau \sigma^{\prime}}\right) \\
& =\frac{1}{2} U\left(\sum_{m \sigma \neq M S} n_{m m}^{T \sigma}+\sum_{m^{\prime} \sigma^{\prime} \neq M S} n_{m^{\prime} m^{\prime}}^{T \sigma^{\prime}}\right) \\
& =U \sum_{m \sigma \neq M S} n_{m m}^{T \sigma}
\end{aligned}
$$

and

$$
\begin{aligned}
V_{d c}=\frac{\partial E_{d c}}{\partial n_{M M}^{T S}} & =\frac{1}{2} U \frac{\partial}{\partial n_{M M}^{T S}} \sum_{\tau} N_{\tau}\left(N_{\tau}-1\right) \\
& =\frac{1}{2} U \frac{\partial}{\partial n_{M M}^{T S}}\left[N_{T}\left(N_{T}-1\right)\right] \\
& =\frac{1}{2} U\left[\left(N_{T}-1\right)+\left(N_{T}\right)\right] \\
& =U\left(N_{T}-\frac{1}{2}\right),
\end{aligned}
$$

where we have used the fact that $\partial N_{T} / \partial n_{M M}^{T S}=1$. Overall our new effective potential is

$$
v_{t o t}=v_{K S}+U \sum_{m \sigma \neq M S} n_{m m}^{T \sigma}-U\left(N_{T}-\frac{1}{2}\right)=v_{K S}+U\left(\frac{1}{2}-n_{M M}^{T S}\right)
$$

This equation tells us the basic behavior of the DFT $+U$ approach. When we have an occupied correlated orbital, $n_{M M}^{T S}$ is near unity so the correction to the potential is $-U / 2$. For an unoccupied correlated orbital, $n_{M M}^{T S}$ is near zero so the correction to the potential is $+U / 2$. Therefore, we expect to open an electronic band gap of around the Coulomb energy $U$. Note that we still solve our modified Kohn-Sham equations iteratively. So the method does not just perform a rigid shift - it takes into account other factors such as changes in 
hybridization between the correlated and noncorrelated orbitals.

\subsubsection{Rotationally-invariant formalism}

While the above formalism captures the essence of the DFT $+U$ method, it turns out to not be invariant to a unitary rotation of the localized atomic basis. So it is preferable in practice to use a rotationally-invariant implementation [Liechtenstein et al. (1995)]. The equations, not derived here, come from Hartree-Fock theory and are as follows:

$$
\begin{aligned}
E_{U} & =\frac{1}{2} \sum_{\{m\}, \sigma, \tau}\left[\left\langle m m^{\prime \prime}\left|V_{e e}\right| m^{\prime} m^{\prime \prime \prime}\right\rangle n_{m m^{\prime}}^{\tau \sigma} n_{m^{\prime \prime} m^{\prime \prime \prime}}^{\tau \overline{\bar{c}}}\right. \\
& \left.+\left(\left\langle m m^{\prime \prime}\left|V_{e e}\right| m^{\prime} m^{\prime \prime \prime}\right\rangle-\left\langle m m^{\prime \prime}\left|V_{e e}\right| m^{\prime \prime \prime} m^{\prime}\right\rangle\right) n_{m m^{\prime}}^{\tau \sigma} n_{m^{\prime \prime} m^{\prime \prime \prime}}^{\tau \sigma}\right]
\end{aligned}
$$

where $V_{e e}=1 /\left|\mathbf{r}-\mathbf{r}^{\prime}\right|$ is the Coulomb potential and we use the notation $\left|m m^{\prime}\right\rangle=\left|\phi_{m}^{\tau}\right\rangle\left|\phi_{m^{\prime}}^{\tau}\right\rangle$.

The matrix elements of $V_{e e}$ are expanded based on spherical harmonics with

$$
\left\langle m m^{\prime \prime}\left|V_{e e}\right| m^{\prime} m^{\prime \prime \prime}\right\rangle=\sum_{k=0}^{2 l} a_{k}\left(m, m^{\prime}, m^{\prime \prime}, m^{\prime \prime \prime}\right) F^{k}
$$

where $l$ is the angular momentum,

$$
a_{k}\left(m, m^{\prime}, m^{\prime \prime}, m^{\prime \prime \prime}\right)=\frac{4 \pi}{2 k+1} \sum_{q=-k}^{+k}\left\langle l m\left|Y_{k q}\right| l m^{\prime}\right\rangle\left\langle l m^{\prime \prime}\left|Y_{k q}^{*}\right| l m^{\prime \prime \prime}\right\rangle
$$

and $F^{k}$ are radial Slater integrals.

The screened (not bare) Coulomb potential is appropriate in this case due to the screening of the noncorrelated degrees of freedom. So in practice we treat the matrix elements of the Coulomb potential as parameters written in terms of $U$ and $J$. Let's take the example of $d$ 
electrons.

$$
\begin{gathered}
U=\frac{1}{(2 l+1)^{2}} \sum_{m, m^{\prime}}\left\langle m m^{\prime}\left|V_{e e}\right| m m^{\prime}\right\rangle=F^{0} \\
J=\frac{1}{2 l(2 l+1)} \sum_{m \neq m^{\prime}}\left\langle m m^{\prime}\left|V_{e e}\right| m^{\prime} m\right\rangle=\frac{F^{2}+F^{4}}{14}
\end{gathered}
$$

Typically we assume $F^{2} / F^{4}$ has the same value as in an isolated atom (0.625). So then we have all the necessary Slater integrals parametrized in terms of $U$ and $J$ and our functional is fully defined. In this formalism our FLL double counting energy is

$$
E_{d c}=\frac{1}{2} U \sum_{\tau} N_{\tau}\left(N_{\tau}-1\right)-\frac{1}{2} J \sum_{\tau, \sigma} N_{\tau \sigma}\left(N_{\tau \sigma}-1\right)
$$

where $N_{\tau \sigma}=\sum_{m} n_{m m}^{\tau \sigma}$.

\subsection{DFT plus dynamical mean-field theory}

\subsubsection{Dynamical mean-field theory}

Dynamical mean-field theory (DMFT) is a newer approach to solve the many-body problem that relies on an approximation of an effective interaction potential that is local in space but is dynamical (i.e., fluctuating in time) [Georges et al. (1996)]. There are a few important foundational papers for DMFT. The first comes from Walter Metzner and Dieter Vollhardt [Metzner and Vollhardt (1989)]. Using the Hubbard model (see Sec. 1.3.1) as an example, they demonstrated that the limit of infinite dimensions, $d \rightarrow \infty$, is non-trivial if the hopping parameter is scaled as $t / \sqrt{d}$ to ensure the energies remain finite. In this limit, common approximations used in variational calculations such as the Gutzwiller approximation become exact. Soon after Erwin Müller-Hartmann proved that the self-energy 
$\Sigma$, which encodes all the interaction effects, is local in space (i.e., it has no dependence on k) in this limit [Müller-Hartmann (1989)]. The final paper comes from Antoine Georges and Gabriel Kotliar [Georges and Kotliar (1992)]. Also working in the context of the Hubbard model, in 1992 they showed that in infinite dimensions this problem can be exactly mapped onto an auxiliary problem known as a quantum impurity problem, with an additional selfconsistency condition. This contribution was also made by Fusayoshi Ohkawa around the same time [Ohkawa (1991a,b)].

A quantum impurity problem corresponds to a single interacting lattice site embedded in a reservoir or bath of non-interacting electrons. In this case, the bath is chosen to mimic the effect of the discarded lattice sites. While the quantum impurity problem is still a manybody problem, it is a drastically simpler one. The relevant quantum impurity problem, the famous Anderson impurity model originally devised in the context of magnetic impurities and Kondo physics [Anderson (1961)], can be described by the effective Hamiltonian

$$
\hat{H}_{\mathrm{eff}}=\sum_{\mathbf{k}, \sigma} \varepsilon_{\mathbf{k}} \hat{n}_{\mathbf{k} \sigma}+\varepsilon_{d} \sum_{\sigma} \hat{n}_{d \sigma}+U \hat{n}_{d \uparrow} \hat{n}_{d \downarrow}+\sum_{\mathbf{k}, \sigma}\left(V_{\mathbf{k} d} \hat{c}_{\mathbf{k} \sigma}^{\dagger} \hat{d}_{\sigma}+\bar{V}_{\mathbf{k} d} \hat{d}_{\sigma}^{\dagger} \hat{c}_{\mathbf{k} \sigma}\right) .
$$

Here $\hat{d}_{\sigma}^{(\dagger)}$ is the annihilation (creation) operator for a correlated orbital of spin $\sigma$, energy $\varepsilon_{d}$, and number operator $\hat{n}_{d \sigma}=\hat{d}_{\sigma}^{\dagger} \hat{d}_{\sigma} ; \hat{c}_{\mathbf{k} \sigma}^{(\dagger)}$ is the bath orbital annihilation (creation) operator for crystal momentum $k$, spin $\sigma$, and energy $\varepsilon_{\mathbf{k}}$ with number operator $\hat{n}_{\mathbf{k} \sigma}$. The first two terms encode the non-interacting band structure of the correlated (e.g. $d$ or $f$ ) and bath states, respectively, while the third term is the on-site Coulomb interaction parametrized by $U$. The final term, called the hybridization, contains the interaction between the bath orbitals and the correlated orbitals via the hybridization parameters $V_{\mathbf{k} d}$. The schematic picture of DMFT is illustrated in Fig. 1.5.

DMFT, which was developed in the context of model Hamiltonians, is written in the language of the single-particle Green function $-i\left\langle\Psi\left|\mathcal{T}\left[\psi(\mathbf{r}, t) \psi^{\dagger}\left(\mathbf{r}^{\prime}, t^{\prime}\right)\right]\right| \Psi\right\rangle$, where $\mathcal{T}$ is the time-ordering operation, $\psi^{(\dagger)}$ is the single-particle annihilation (creation) operator, and $\Psi$ is 


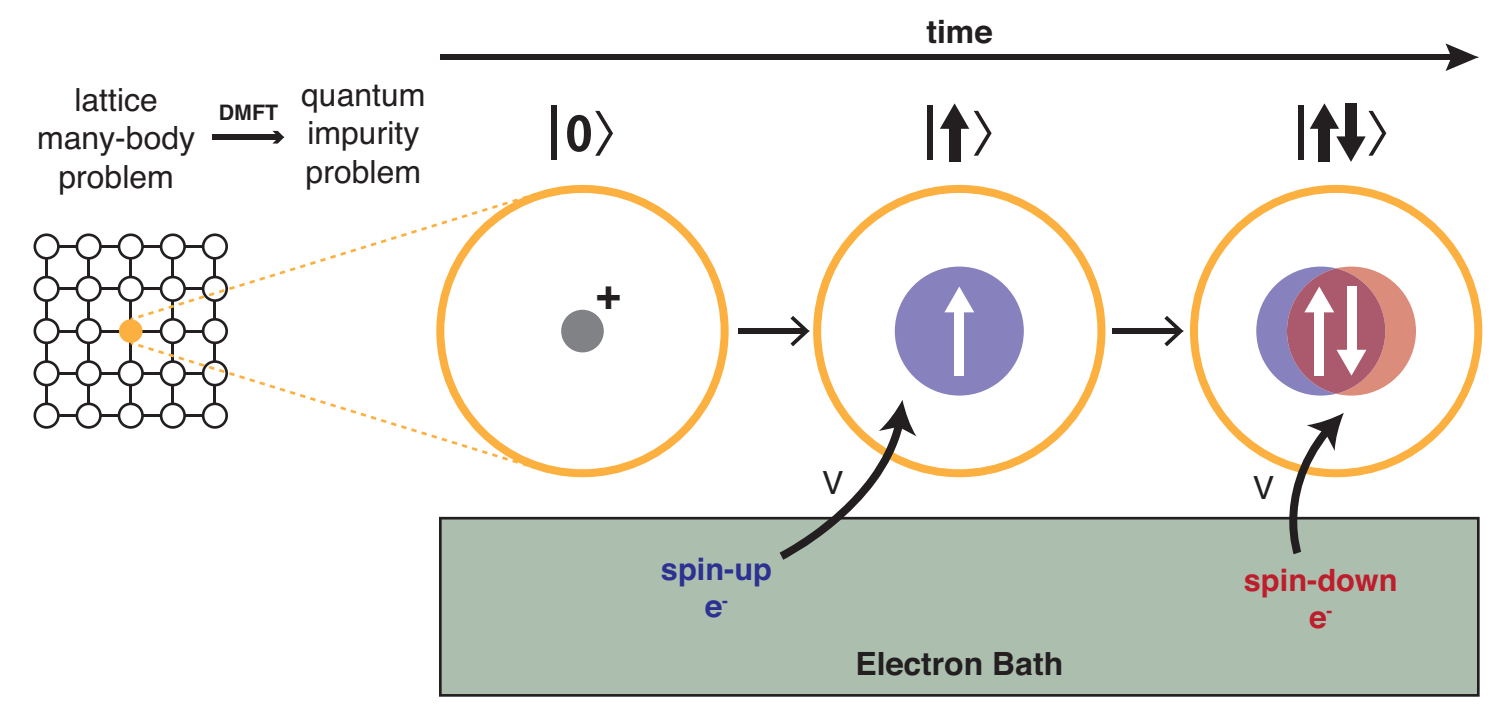

Figure 1.5: DMFT maps the lattice many-body problem onto a quantum impurity problem of a single lattice site embedded in a fictitious bath of electrons chosen to mimic the effect of the discarded lattice sites. DMFT, which is exact in the limit of infinite dimensions, captures the dynamical local correlations. In the right part of this schematic, a single correlated orbital is considered. As a function of time, electrons can hop on and off of the site via hybridization with the bath. The concept of this image comes from Kotliar and Vollhardt (2004). 
the many-body wavefunction. In this language, working in the momentum and frequency domain, the self-consistency condition of DMFT is the requirement that the Green function of the impurity problem $G(\omega)$ equals the local lattice Green function $G_{\tau \tau}(\omega)$ :

$$
G(\omega)=G_{\tau \tau}(\omega)=\frac{1}{N_{\mathbf{k}}} \sum_{\mathbf{k}} \frac{1}{\omega+\mu-\varepsilon_{\mathbf{k}}-\Sigma(\omega)}
$$

where the $\mathbf{k}$-sum is over the $N_{\mathbf{k}} k$-points in the first Brillouin zone, $\omega$ is the frequency, and $\mu$ is the chemical potential. The hybridization parameters in $\hat{H}_{\text {eff }}$ are chosen such that this self-consistency condition is satisfied. The self-energy $\Sigma$ contains all of the interaction effects and can be determined by the Dyson equation

$$
\mathcal{G}^{-1}(\omega)=G^{-1}(\omega)+\Sigma(\omega)
$$

where $\mathcal{G}$ is the bath Green function. The real part of the self-energy dictates shifts in the quasiparticle energy levels, whereas the imaginary part relates to the broadening and incoherence of these states.

The fundamental approximation of DMFT is a local (i.e., $\mathbf{k}$-independent) self-energy $\Sigma$. As indicated above, the self-energy above only depends on frequency $\omega$ and is identical for each k. Therefore, DMFT captures dynamical local correlations (as opposed to only static local correlations in Hartree-Fock theory). This approximation becomes exact in the limit of infinite dimension or lattice coordination, and there is evidence that this is generally a reasonable approximation even in finite (e.g. two or three) dimensions [Georges et al. (1996)]. In cases in which nonlocal correlations are relevant, there are cluster extensions to DMFT [Maier et al. (2005)] that can recover the momentum dependence of $\Sigma$, though we will not discuss them here. 


\subsubsection{Merging DFT and dynamical mean-field theory}

$\mathrm{DFT}+\mathrm{DMFT}$ is analogous to $\mathrm{DFT}+U$, but it employs DMFT rather than the HartreeFock approximation to solve the correlation problem [Kotliar et al. (2006)]. We note that $\mathrm{DFT}+\mathrm{DMFT}$ is a nonperturbative approach, unlike other beyond-DFT approaches such as the $G W$ approximation. Here we focus on the total energy formalism of Park et al. (2014), which is employed in Chap. 3. In this formalism, the Wannier function basis is employed and the DMFT equations are solved on the axis of imaginary Matsubara frequencies $i \omega_{n}$. The total energy becomes a functional of the single-particle Green function $G$ in addition to $\rho:$

$$
E_{D F T+D M F T}[\rho, G]=E_{D F T}[\rho]+E_{K S}[\rho, G]+E_{U}[G]-E_{d c}[G]
$$

As the single-particle Green function contains much more information than the single-particle density matrix, the additional fundamental variable of $\mathrm{DFT}+U$, DFT $+\mathrm{DMFT}$ is a substantially more complex electronic structure theory. The interaction energy is computed via

$$
E_{U}=\frac{1}{2} T \sum_{n} e^{i \omega_{n} 0^{-}} \operatorname{Tr}\left[\Sigma\left(i \omega_{n}\right) G\left(i \omega_{n}\right)\right]
$$

where $T$ is the temperature. Here $G$ and $\Sigma$ are matrices in the space of correlated orbitals, and we are assuming only a single correlated site $\tau$ for convenience. The double counting energy, which is identical to that of $\mathrm{DFT}+U$, is still only a function of $N$ and is obtained from $G$ via the density matrix $n=\left(1 / N_{\mathbf{k}}\right) \sum_{\mathbf{k}} T \sum_{i \omega_{n}} G_{\mathbf{k}}\left(i \omega_{n}\right) e^{i \omega_{n} 0^{-}}$, where $G_{\mathbf{k}}(\omega)=[\omega+$ $\left.\mu-\varepsilon_{\mathbf{k}}-\Sigma(\omega)\right]^{-1} . E_{K S}[\rho, G]$ is a correction term for the band energy, which is needed since the density matrix component $n_{\mathbf{k} \nu}$ for crystal momentum $\mathbf{k}$ and band $\nu$ differs from the Fermi factor $f_{\mathbf{k} \nu}$, and takes the form

$$
E_{K S}[\rho, G]=\frac{1}{N_{\mathbf{k}}} \sum_{\mathbf{k} \nu} \varepsilon_{\mathbf{k} \nu}^{K S}\left(n_{\mathbf{k} \nu}-f_{\mathbf{k} \nu}\right),
$$

where $\varepsilon_{\mathbf{k} \nu}^{K S}$ is the Kohn-Sham eigenvalue. 
Here we focus on the rotationally-invariant Slater-Kanamori form of the interaction term, as that will be used later in Chapter 3. This Hamiltonian can be split into components that are diagonal (D) and off-diagonal (OD) in the density via $\hat{H}=\hat{H}_{D}+\hat{H}_{O D}$. The diagonal part of the Hamiltonian is

$$
\begin{aligned}
\hat{H}_{D} & =U \sum_{m} \hat{n}_{m \uparrow} \hat{n}_{m \downarrow}+(U-2 J) \sum_{m \neq m^{\prime}} \hat{n}_{m \uparrow} \hat{n}_{m^{\prime} \downarrow}+(U-3 J) \sum_{m>m^{\prime}, \sigma} \hat{n}_{m \sigma} \hat{n}_{m^{\prime} \sigma} \\
& =\frac{1}{2} U\left(\hat{N}^{2}-\hat{N}\right)-\frac{3}{2} J \sum_{\sigma}\left(\hat{N}_{\sigma}^{2}-\hat{N}_{\sigma}\right)-J \sum_{\sigma}\left(\hat{N}_{\sigma} \hat{N}_{\bar{\sigma}}-\sum_{m} \hat{n}_{m \sigma} \hat{n}_{m \bar{\sigma}}\right)
\end{aligned}
$$

where $\hat{N}=\sum_{\alpha, \sigma} \hat{n}_{\alpha \sigma}$ and $\hat{N}_{\sigma}=\sum_{\alpha} \hat{n}_{\alpha \sigma}$. The off-diagonal component is

$$
\hat{H}_{O D}=J \sum_{m \neq m^{\prime}}\left(\hat{\psi}_{m \uparrow}^{\dagger} \hat{\psi}_{m^{\prime} \uparrow} \hat{\psi}_{m^{\prime} \downarrow}^{\dagger} \hat{\psi}_{m \downarrow}+\hat{\psi}_{m \uparrow}^{\dagger} \hat{\psi}_{m^{\prime} \uparrow} \hat{\psi}_{m \downarrow}^{\dagger} \hat{\psi}_{m^{\prime} \downarrow}\right)
$$

and corresponds to the double exchange and pair hopping interactions. To compute the mean-field (Hartree-Fock) part of the interaction energy, we take the expectation value of 
$\hat{H}_{D}$ and apply the Hartree-Fock approximation:

$$
\begin{aligned}
& \left\langle\hat{H}_{D}\right\rangle=\frac{1}{2} U\left\langle\hat{N}^{2}-\hat{N}\right\rangle-\frac{3}{2} J \sum_{\sigma}\left\langle\hat{N}_{\sigma}^{2}-\hat{N}_{\sigma}\right\rangle-J \sum_{\sigma}\left(\hat{N}_{\sigma} \hat{N}_{\bar{\sigma}}-\sum_{m} \hat{n}_{m \sigma} \hat{n}_{m \bar{\sigma}}\right) \\
& =\frac{1}{2} U\left\langle\left(\sum_{m, \sigma} \hat{n}_{\sigma m}\right)^{2}-\left(\sum_{m, \sigma} \hat{n}_{\sigma m}^{2}\right)\right\rangle-\frac{3}{2} J \sum_{\sigma}\left\langle\left(\sum_{m} \hat{n}_{\sigma m}\right)^{2}-\left(\sum_{m} \hat{n}_{\sigma m}^{2}\right)\right\rangle \\
& -J \sum_{\sigma}\left(\left\langle\sum_{m} \hat{n}_{m \sigma} \sum_{m^{\prime}} \hat{n}_{m^{\prime} \bar{\sigma}}\right\rangle-\left\langle\sum_{m} \hat{n}_{m \sigma} \hat{n}_{m \bar{\sigma}}\right\rangle\right) \\
& =\frac{1}{2} U\left(\sum_{m, m^{\prime}, \sigma, \sigma^{\prime}}\left\langle\hat{n}_{m \sigma} \hat{n}_{m^{\prime} \sigma^{\prime}}\right\rangle-\sum_{m, \sigma}\left\langle\hat{n}_{m \sigma}^{2}\right\rangle\right)-\frac{3}{2} J \sum_{\sigma}\left\langle\sum_{m, m^{\prime}} \hat{n}_{m \sigma} \hat{n}_{m^{\prime} \sigma}-\sum_{m} \hat{n}_{m \sigma}^{2}\right\rangle \\
& -J \sum_{\sigma}\left\langle\sum_{m, m^{\prime}} \hat{n}_{m \sigma} \hat{n}_{m^{\prime} \bar{\sigma}}-\sum_{m} \hat{n}_{m \sigma} \hat{n}_{m \bar{\sigma}}\right\rangle \\
& E_{M F}^{c o r}=\frac{1}{2} U\left(\sum_{m, m^{\prime}, \sigma, \sigma^{\prime}}\left\langle\hat{n}_{m \sigma}\right\rangle\left\langle\hat{n}_{m^{\prime} \sigma^{\prime}}\right\rangle-\sum_{m, \sigma}\left\langle\hat{n}_{m \sigma}\right\rangle^{2}\right)-\frac{3}{2} J\left(\sum_{m, m^{\prime}, \sigma}\left\langle\hat{n}_{m \sigma}\right\rangle\left\langle\hat{n}_{m^{\prime} \sigma}\right\rangle-\sum_{m, \sigma}\left\langle\hat{n}_{m \sigma}\right\rangle^{2}\right) \\
& -J\left(\sum_{m, m^{\prime}, \sigma}\left\langle\hat{n}_{m \sigma}\right\rangle\left\langle\hat{n}_{m^{\prime} \bar{\sigma}}\right\rangle-\sum_{m, \sigma}\left\langle\hat{n}_{m \sigma}\right\rangle\left\langle\hat{n}_{m \bar{\sigma}}\right\rangle\right) \\
& =\frac{1}{2} U\left[\left(\sum_{m, \sigma}\left\langle\hat{n}_{m \sigma}\right\rangle\right)^{2}-\left(\sum_{m, \sigma}\left\langle\hat{n}_{m \sigma}\right\rangle^{2}\right)\right]-\frac{3}{2} J \sum_{\sigma}\left[\left(\sum_{m}\left\langle\hat{n}_{m \sigma}\right\rangle\right)^{2}-\left(\sum_{m}\left\langle\hat{n}_{m \sigma}\right\rangle^{2}\right)\right] \\
& -J \sum_{\sigma}\left(\sum_{m}\left\langle\hat{n}_{m \sigma}\right\rangle \sum_{m^{\prime}}\left\langle\hat{n}_{m^{\prime} \bar{\sigma}}\right\rangle-\sum_{m}\left\langle\hat{n}_{m \sigma}\right\rangle\left\langle\hat{n}_{m \bar{\sigma}}\right\rangle\right) \\
& =\frac{1}{2} U\left(N^{2}-\sum_{m, \sigma}\left\langle\hat{n}_{m \sigma}\right\rangle^{2}\right)-\frac{3}{2} J\left(\sum_{\sigma} N_{\sigma}^{2}-\sum_{m, \sigma}\left\langle\hat{n}_{m \sigma}\right\rangle^{2}\right)-J \sum_{\sigma}\left(N_{\sigma} N_{\bar{\sigma}}-\sum_{m}\left\langle\hat{n}_{m \sigma}\right\rangle\left\langle\hat{n}_{m \bar{\sigma}}\right\rangle\right)
\end{aligned}
$$

To derive the mean-field part of the corresponding potential $V_{M F}^{c o r}$, we take the meanfield correlation energy expression above and take the derivative with respect to a particular 
$\left\langle\hat{n}_{m \sigma}\right\rangle$ labeled by $m=M$ and $\sigma=s$ :

$$
\begin{aligned}
V_{M F}^{c o r} & =\frac{\partial E_{M F}^{c o r}}{\partial\left\langle\hat{n}_{M s}\right\rangle} \\
& =\frac{U}{2}\left(2 N-2\left\langle\hat{n}_{M s}\right\rangle\right)-\frac{3 J}{2}\left(2 N_{s}-2\left\langle\hat{n}_{M s}\right\rangle\right)-J\left[\left(N_{\bar{s}}+N_{\bar{s}}\right)-\left(\left\langle\hat{n}_{M \bar{s}}\right\rangle+\left\langle\hat{n}_{M \bar{s}}\right\rangle\right)\right] \\
& =U\left(N-\left\langle\hat{n}_{M s}\right\rangle\right)-3 J\left(N_{s}-\left\langle\hat{n}_{M s}\right\rangle\right)-J\left(2 N_{\bar{s}}-2\left\langle\hat{n}_{M \bar{s}}\right\rangle\right) \\
& =U\left(N-\left\langle\hat{n}_{M s}\right\rangle\right)-3 J\left(N_{s}-\left\langle\hat{n}_{M s}\right\rangle\right)-2 J\left(N_{\bar{s}}-\left\langle\hat{n}_{M \bar{s}}\right\rangle\right)
\end{aligned}
$$

Assuming paramagnetism, $\left\langle\hat{n}_{m \sigma}\right\rangle=\left\langle\hat{n}_{m \bar{\sigma}}\right\rangle \equiv n_{m} \forall m$ and $N_{\sigma}=N_{\bar{\sigma}} \equiv N / 2$. This gives

$$
\begin{aligned}
V_{M F}^{c o r} & =U\left(N-n_{m}\right)-3 J\left(\frac{1}{2} N-n_{m}\right)-2 J\left(\frac{1}{2} N-n_{m}\right) \\
& =U\left(N-n_{m}\right)-5 J\left(\frac{1}{2} N-n_{m}\right) \\
& =U\left(N-n_{m}\right)-2.5 J\left(N-2 n_{m}\right) \\
& =U\left(N-2 n_{m}\right)+U n_{m}-2.5 J\left(N-2 n_{m}\right) \\
& =(U-2.5 J)\left(N-2 n_{m}\right)+U n_{m}
\end{aligned}
$$

Using the Slater-Kanamori interaction, the FLL double counting energy becomes

$$
E_{d c}=\frac{U}{2} N(N-1)-\frac{5 J}{4} N(N-2)
$$

In order to capture dynamical correlation effects, one must utilize an impurity solver that goes beyond the Hartree-Fock approximation. In this thesis we employ the continuoustime quantum Monte Carlo (CTQMC) impurity solver, which relies on the diagrammatic expansion of the partition function $Z$ for the impurity problem and a stochastic sampling of all the local Feynman diagrams [Gull et al. (2011)]. CTQMC is a numerically-exact, finitetemperature approach in which the DMFT equations are solved on the imaginary frequency axis. We utilize the hybridization expansion type of CTQMC [Werner and Millis (2006); 
Werner et al. (2006); Haule (2007)], for which the partition function is expanded in the partition function from the atomic limit.

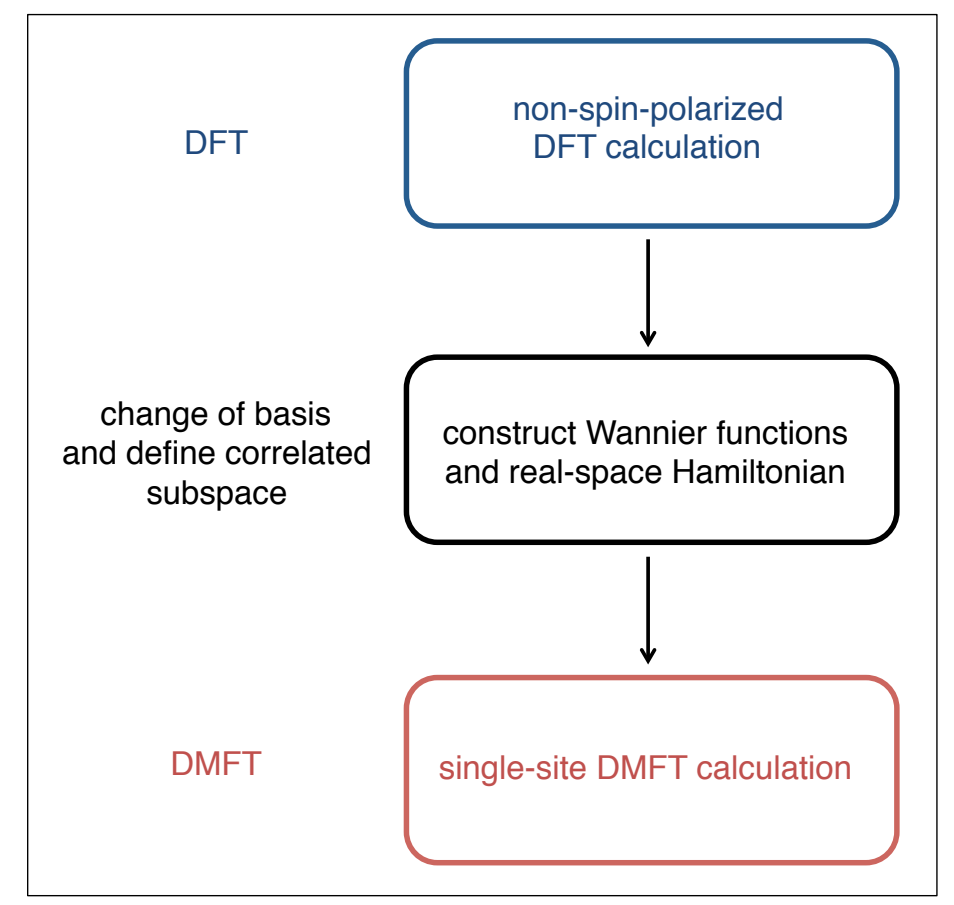

Figure 1.6: Overall workflow for the DFT+DMFT calculations.

Figure 1.6 illustrates the basic workflow of our single-site, paramagnetic DFT+DMFT approach. First a non-spin-polarized DFT calculation is performed. Then the Wannier functions are computed and the real-space Hamiltonian is computed. Finally, we perform the single-site DMFT calculation based on this Hamiltonian.

A workflow for the DMFT component is shown in Fig. 1.7. The non-interacting properties are first computed by setting the self-energy $\Sigma$ to zero. Then the Hartree-Fock approximation is taken as an initial guess for $\Sigma$ and the hybridization function $\Delta$ encoding the hybridization parameters is computed based on this $\Sigma$ and the Green function. At this 
1. Introduction

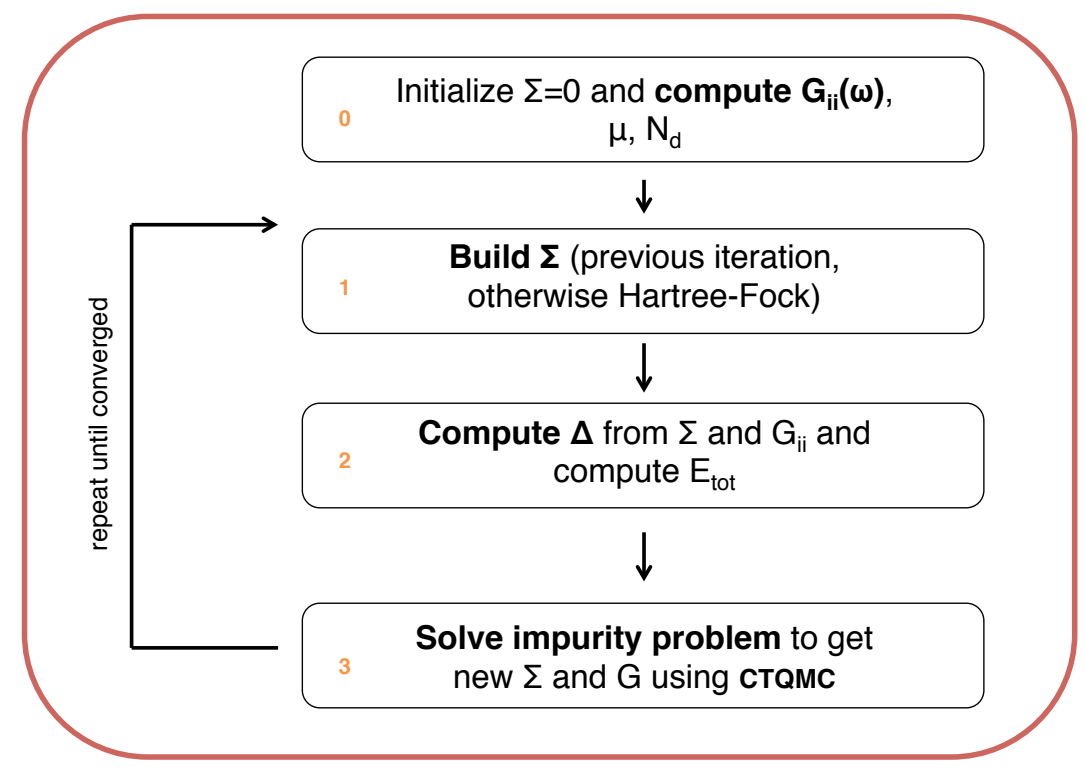

Figure 1.7: DMFT component of the workflow for DFT+DMFT calculations. 
step the total energy computation is performed. CTQMC is then employed to solve the impurity problem to obtain the new Green function and self-energy for the next iteration of the self-consistency loop. Note that here there is no charge self-consistency, so there is no outer loop to recompute the DFT properties and Wannier functions. 
2. Phase stability of strongly correlated electron materials within $D F T+U$

\section{Chapter 2}

\section{Phase stability of strongly correlated electron materials within DFT $+U$}

Predicting the compositional phase stability of correlated transition metal oxides and phosphates is an outstanding challenge in condensed matter physics. In this work, we employ the density functional theory plus $U(\mathrm{DFT}+U)$ formalism to address the effects of local correlations arising from transition metal $d$ electrons in the prototype materials layered $\mathrm{Li}_{x} \mathrm{CoO}_{2}$ and olivine $\mathrm{Li}_{x} \mathrm{FePO}_{4}$. We exploit a new spectral representation of the DFT $+U$ total energy revealing the distinct roles of the filling and ordering of the correlated subspace. The on-site interaction $U$ drives both of these very different materials systems towards phase separation, stemming from enhanced ordering of the $d$ orbital occupancies in the $x=0$ and $x=1$ species, whereas changes in the overall filling of the $d$ shell contribute negligibly. Charge ordering and structural relaxations dampen, but do not eliminate, this tendency to phase separate. We show that $\mathrm{DFT}+U$ is qualitatively consistent with experiments for phase stable $\mathrm{Li}_{x} \mathrm{CoO}_{2}$, phase separating $\mathrm{Li}_{x} \mathrm{FePO}_{4}$, and phase stable $\mathrm{Li}_{x} \mathrm{CoPO}_{4}$. A related observable which probes the accuracy of total energies within $\mathrm{DFT}+U$ is the Li order-disorder transition temperature for $\mathrm{Li}_{1 / 2} \mathrm{CoO}_{2}$, and we find a substantial overestimation of this observable stemming from unphysical charge ordering arising from the correlated subspace interaction energy as opposed to the double counting. More generally, DFT $+U$ tends to predict charge ordering in nonstochiometric compounds, even for a wide range of composition, raising the difficult question of when this effect is physical. We demonstrate that the phase stability of $\mathrm{Li}_{1 / 2} \mathrm{CoO}_{2}$ within $\mathrm{DFT}+U$ is qualitatively incorrect without strong charge ordering, resulting 
in a large band gap, which is not observed in experiment. Our results motivate the need for other advanced techniques, such as DFT plus dynamical mean-field theory, for total energies in strongly correlated materials.

\section{$2.1 \quad$ Introduction}

Strongly correlated materials (SCMs), in which density functional theory (DFT) [Hohenberg and Kohn (1964); Kohn and Sham (1965)] calculations break down for selected observables due to strong electron-electron interactions, are at the forefront of condensed matter physics [Kotliar and Vollhardt (2004); Morosan et al. (2012)]. Phenomenologically, SCMs exist in a ground state which is in the vicinity of a Mott transition [Mott (1968); Imada et al. (1998)] whereby electronic hopping may be overwhelmed by local interactions, resulting in an insulating state. Realizations of SCMs often contain atoms with open shell $d$ or $f$ electrons, such as the high-temperature superconducting cuprates [Bednorz and Müller (1986)], colossal magnetoresistance manganites [Ramirez (1997)], and heavy fermion actinide based materials [Coleman (2007)].

Predicting the properties of strongly correlated materials is an outstanding problem in solid state physics. The standard approach of DFT, which is the most generic theory of electronic structure for materials physics, is in principle an exact theory for the ground state energy and electron density of any many-electron system. However, the exact exchangecorrelation functional needed is unknown and typically is approximated based on the homogeneous electron gas. As a result, DFT in practice is typically unable to capture critical aspects of the physics of strongly correlated materials [Jones and Gunnarsson (1989); Jones (2015); Kotliar and Vollhardt (2004)].

One of many important technological contexts highlighting the need for an accurate electronic structure method for strongly correlated materials is that of Li ion batteries. These

electrochemical cells, which enable energy storage via the reversible transport (cycling) of 
Li ions between two electrodes through an electrolyte, rely critically on a cathode material that is able to intercalate Li ions while maintaining the same structure. Cathode materials typically employ transition metals to accommodate the changes in oxidation state associated with the presence or absence of Li ions. They usually also contain oxygen or another oxygencontaining anion group. Since they have an open $d$ electron shell, cathode materials are often susceptible to strong correlation physics.

Many of the current cathode materials utilized today are based on the material $\mathrm{Li}_{x} \mathrm{CoO}_{2}$, shown in Fig. 2.1(a) for $x=1$, which can store Li ions in between its $\mathrm{CoO}_{2}$ layers [Mizushima et al. (1980)]. Although cathodes based on $\mathrm{Li}_{x} \mathrm{CoO}_{2}$ have been very commercially successful, there is currently strong demand for new cathodes with increased energy capacity, decreased charge/discharge time, reinforced safety, and reduced cost as Li ion batteries enter large-scale applications such as electric vehicles and grid-level energy storage. [Ellis et al. (2010)] To help achieve to this goal, significant efforts are being made to computationally screen new candidates for rechargeable battery cathodes [Ceder (2010); Hautier et al. (2011b); Mueller et al. (2011); Hautier et al. (2011a)].

One of the most fundamental properties of a potential battery cathode material is whether there are stable compounds of intermediate Li concentration $(0<x<1)$. This property, called phase stability, has a strong impact on the charge/discharge mechanism and also can impact the voltage and capacity of the battery. For example, the fast charge/discharge kinetics and $x$-dependent voltage in $\mathrm{Li}_{x} \mathrm{CoO}_{2}$ stem from its several stable intermediate phases [Reimers and Dahn (1992); Van der Ven et al. (1998); Wolverton and Zunger (1998)]. Irreversible phase transitions when delithiating to $x<0.5$, for which the stable compounds have significant structural differences, unfortunately limit the usable capacity of $\mathrm{Li}_{x} \mathrm{CoO}_{2}$ to $x>0.5$ [Whittingham (2004)].

Exhibiting high thermal stability, voltage, and theoretical capacity and consisting of Earth-abundant elements, olivine $\mathrm{Li}_{x} \mathrm{FePO}_{4}$ [see Fig. 2.1(b)] is a promising candidate for battery cathodes [Padhi et al. (1997b,a)]. However, in contrast to $\mathrm{Li}_{x} \mathrm{CoO}_{2}, \mathrm{Li}_{x} \mathrm{FePO}_{4}$ does 
(a) $\mathrm{Li}_{x} \mathrm{CoO}_{2}$

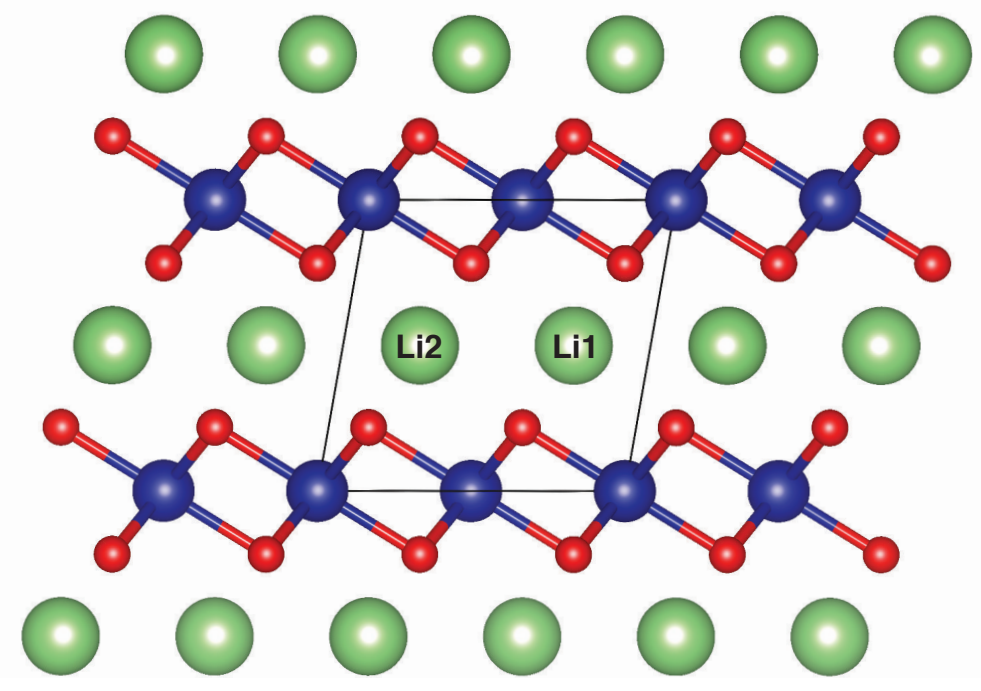

(b) $\mathrm{Li}_{x} \mathrm{FePO}_{4}$

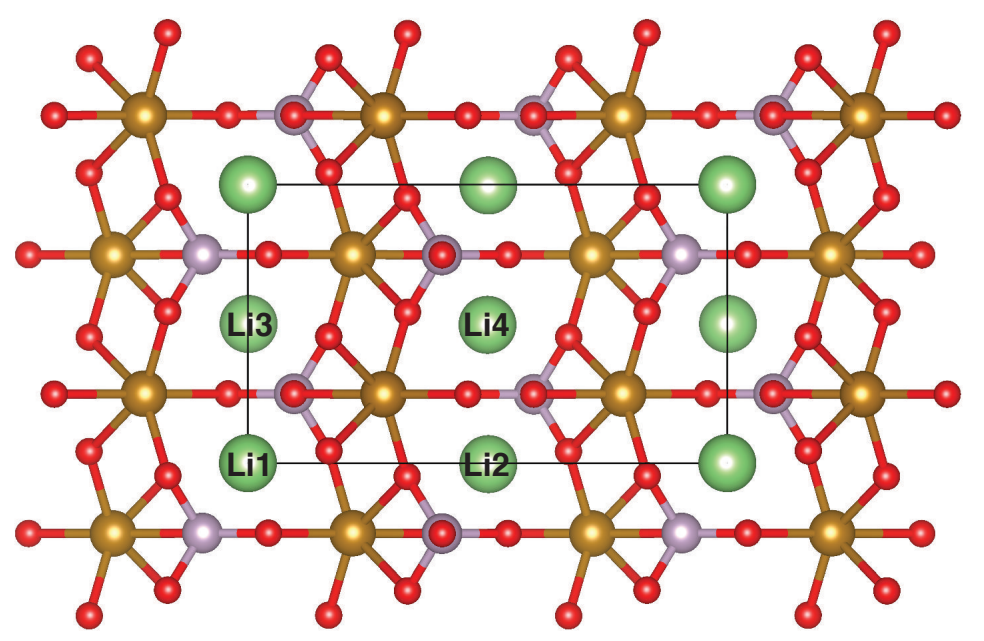

Figure 2.1: Crystal structures of (a) $\mathrm{Li}_{x} \mathrm{CoO}_{2}$ and (b) $\mathrm{Li}_{x} \mathrm{FePO}_{4}$. The large green, medium blue, medium gold, small purple, and small red spheres represent ionic positions of $\mathrm{Li}, \mathrm{Co}, \mathrm{Fe}, \mathrm{P}$, and $\mathrm{O}$, respectively The black lines indicate for (a) the unit cell of the lowest-energy $\mathrm{Li}_{1 / 2} \mathrm{CoO}_{2}$ configuration and for (b) the primitive unit cell. Images of crystal structures are generated using VESTA [Momma and Izumi (2011)]. 
not have any stable compounds for intermediate $x$. It instead exhibits phase separation into its fully lithiated $(x=0)$ and fully delithiated $(x=1)$ endmembers, which may contribute to slow charge/discharge kinetics. [Padhi et al. (1997b); Delacourt et al. (2005); Dodd et al. (2006)] While nanostructuring $\mathrm{Li}_{x} \mathrm{FePO}_{4}$ can reduce this phase separation at room temperature, [Yamada et al. (2005, 2006); Meethong et al. (2007)] it is generally accompanied by an undesirable loss in volumetric and gravimetric energy and power densities.

G. Ceder and coworkers performed several studies on the phase stability of $\mathrm{Li}_{x} \mathrm{FePO}_{4}$ using electronic structure calculations. DFT predicts very stable compounds of intermediate $x$ in $\mathrm{Li}_{x} \mathrm{FePO}_{4}$, in stark disagreement with experiment [Zhou et al. (2004c)]. In contrast, DFT does not fail to capture the phase stability of $\mathrm{Li}_{x} \mathrm{CoO}_{2}$ [Van der Ven et al. (1998); Wolverton and Zunger (1998)]. A beyond-DFT approach combining DFT and exact HartreeFock exchange, called a hybrid functional, also fails to capture the total phase separation in $\mathrm{Li}_{x} \mathrm{FePO}_{4}$ [Ong et al. (2011)]. DFT $+U$ calculations, which include an explicit on-site Coulomb interaction $U$ for the transition metal $d$ electrons, were found to predict phase separation in $\mathrm{Li}_{x} \mathrm{FePO}_{4}$ for sufficiently high values of $U$ [Zhou et al. (2004c)]. In later work, a cluster expansion based on such $\mathrm{DFT}+U$ energetics rationalized this phase separation in terms of strong Li-electron interactions [Zhou et al. (2006)].

The DFT $+U$ study claims that the instability of compounds of intermediate $x$ for $\mathrm{Li}_{x} \mathrm{FePO}_{4}$ relates to energy penalties stemming from charge ordering (CO), a symmetry breaking in which the number of electrons on different transition metal sites differs [Zhou et al. (2004c)]. However, it is unclear if the CO is physical since this type of static ordering is the only way in which a static mean-field theory like DFT $+U$ can mimic a Mott insulating state. Furthermore, the phase separation in $\mathrm{Li}_{x} \mathrm{FePO}_{4}$ is fundamentally puzzling since with reasonable parameters the canonical model Hamiltonians describing strong correlations such as the Hubbard and $t-J$ models do not exhibit phase separation for extended regions of the phase diagram [Visscher (1974); Marder et al. (1990)].

To explore such issues, in this work we employ extensive DFT $+U$ calculations to un- 
derstand in detail the impact of electronic correlations on phase stability in correlated intercalation materials. We focus on phase stable $\mathrm{Li}_{x} \mathrm{CoO}_{2}$ and phase separating $\mathrm{Li}_{x} \mathrm{FePO}_{4}$ and explicitly investigate the role of $\mathrm{CO}$ and structural relaxations. A new energy decomposition is elucidated to quantitatively analyze the impact of $U$ on the overall filling and on the ordering of orbitals within the $d$ shell. Comparison is made to the $\mathrm{Li}_{x} \mathrm{CoPO}_{4}$ system, which is isostructural to $\mathrm{Li}_{x} \mathrm{FePO}_{4}$ but does have a stable compound of intermediate $x$. We also investigate another physical observable, the order-disorder transition temperature, for $\mathrm{Li}_{1 / 2} \mathrm{CoO}_{2}$ to provide a clear benchmark of $\mathrm{DFT}+U$ theory. The tendency for $\mathrm{CO}$ in $\mathrm{DFT}+U$, how it impacts these thermodynamic properties, and whether it is physical or not is investigated and discussed. A thorough understanding at the $\mathrm{DFT}+U$ level of theory is critical as we advance to more sophisticated methods to study strongly correlated materials.

For all the materials we have investigated, we find that $U$ destabilizes compounds of intermediate $x$. The Coulomb interaction most strongly affects the endmembers by enhancing the deviations in the $d$ orbital occupancies from their average value. The compounds of intermediate $x$ experience the same effect but are unable to orbitally polarize to the same extent, which directly drives them towards phase separation as $U$ increases. CO and structural relaxations serve to dampen, but not eliminate, this fundamental effect. CO leads to a severe overestimation of the order-disorder transition temperature of $\mathrm{Li}_{1 / 2} \mathrm{CoO}_{2}$ and along with experimental evidence suggests it is an unphysical artifact of DFT $+U$. Our calculations demonstrate that the interaction term rather than the double counting term in $\mathrm{DFT}+U$ is responsible for the $\mathrm{CO}$, which suggests that more accurate approaches such as DFT plus dynamical mean-field theory (DFT+DMFT) may be necessary to accurately describe this class of materials.

The rest of this paper is organized as follows. Section 2.2 discusses the $\mathrm{DFT}+U$ approach to materials with strong electronic correlations and elucidates our new energy decomposition in this framework. Section 2.3 describes the computational details for the simulations performed in this work. The electronic structure of the endmembers of $\mathrm{Li}_{x} \mathrm{CoO}_{2}$ and $\mathrm{Li}_{x} \mathrm{FePO}_{4}$ 
within DFT are described in Sec. 2.4.1. The impact of $U$ on the electronic structure of $\mathrm{Li}_{x} \mathrm{CoO}_{2}$ is discussed in Sec. 2.4.2. The origin of the tendency for $\mathrm{CO}$ in DFT $+U$, taking $\mathrm{Li}_{x} \mathrm{CoO}_{2}$ as an example, is the focus of Sec. 2.4.3. The impact of $U$ on the phase stability of $\mathrm{Li}_{x} \mathrm{CoO}_{2}$ is discussed in Sec. 2.4.4. The electronic structure and phase stability of $\mathrm{Li}_{x} \mathrm{FePO}_{4}$ within DFT $+U$ are described in Sec. 2.4.5 and Sec. 2.4.6, respectively. Section 2.4.7 discusses the formation energy trends for $\mathrm{Li}_{x} \mathrm{CoPO}_{4}$. Section 2.4.8 elucidates to role of the double counting on the formation energy trends. The average intercalation voltages for $\mathrm{Li}_{x} \mathrm{CoO}_{2}$ and $\mathrm{Li}_{x} \mathrm{FePO}_{4}$ are presented in Sec. 2.4.9. Section 2.4.10 focuses on the orderdisorder transition temperature of $\mathrm{Li}_{1 / 2} \mathrm{CoO}_{2}$ and its dependence on CO. Finally, conclusions are presented in Sec. 2.5.

\subsection{Methodology}

\subsubsection{DFT $+U$ approach for correlated materials}

The idea of DFT $+U$ [Himmetoglu et al. (2014)] is to provide an improved treatment of electronic correlations by using not only the density $\rho$ as a primary variable of the energy functional, but also the single-particle density matrix of a relevant set of local orbitals associated with strong correlations. This set of orbitals, which form the correlated subspace, are typically localized electronic states having $d$ or $f$ character and in practice are defined using Wannier functions or atomic orbitals $\left|\phi_{m}^{\tau}\right\rangle$ labeled by ionic site $\tau$ and angular momentum projection $m$.

Having defined the correlated subspace, one needs to construct an approximation for the energy as a functional of the density and the single-particle density matrix of the correlated subspace. This is typically approximated using two separate additive functionals:

$$
E_{D F T+U}\left[\rho, n^{\tau s}\right]=E_{D F T}[\rho]+E_{U}\left[n^{\tau s}\right]-E_{d c}\left[n^{\tau s}\right]
$$


where $n^{\tau s}$ is the local single-particle density matrix for spin projection $s$ and $E_{D F T}[\rho]$ is the usual Kohn-Sham DFT energy functional using one of the many possible approximations to the exchange-correlation energy such as the local density approximation (LDA) [Ceperley and Alder (1980); Vosko et al. (1980); Perdew and Zunger (1981); Perdew and Wang (1992)] or the generalized gradient approximation (GGA) [Perdew et al. (1992, 1996)]. The functional of the density matrix $E_{U}\left[n^{\tau s}\right]$, to be defined mathematically below, is given by the Hartree-Fock interaction energy based upon a set of screened interactions within the correlated subspace. There is a clear double counting $(\mathrm{dc})$ problem with such a formulation, as the LDA or GGA already accounts for interactions of the density arising from the correlated subspace, and therefore a double-counting energy $E_{d c}\left[n^{\tau s}\right]$ must be defined and subtracted.

It is common to employ the spin-dependent formulation of DFT (SDFT) rather than pure DFT, in which case the total energy expression becomes

$$
\begin{aligned}
E_{S D F T+U}\left[\rho^{s}, n^{\tau s}\right]= & E_{S D F T}\left[\rho^{s}\right] \\
& +E_{U}\left[n^{\tau s}\right]-E_{d c}\left[n^{\tau s}\right]
\end{aligned}
$$

where $\rho^{s}$ is the density for spin projection $s$. In the simplified rotationally-invariant formalism of Dudarev et al. [Dudarev et al. (1998)] the interaction term, which we always write in the diagonal eigenbasis of the density matrix, is

$$
E_{U}\left[n^{\tau s}\right]=\frac{1}{2} U \sum_{\tau, m s \neq m^{\prime} s^{\prime}} n_{m}^{\tau s} n_{m^{\prime}}^{\tau s^{\prime}}
$$

where $n_{m}^{\tau s}$ is the $m$ th eigenvalue of $n^{\tau s}$ and $U$ represents the screened on-site Coulomb energy between different spin-orbitals within the correlated subspace. This approach is equivalent to the full rotationally-invariant formalism by Liechtenstein et al. [Liechtenstein et al. (1995)] if the on-site exchange parameter $J$ is set to 0 , which can be justified by previous work indicating that SDFT already contains an intrinsic $J$ [Chen et al. (2015); Park et al. (2015)]. 
The most commonly used dc is the fully-localized-limit (FLL) type

$$
E_{d c}\left[n^{\tau s}\right]=\frac{1}{2} U \sum_{i} N^{\tau}\left(N^{\tau}-1\right),
$$

in which $N^{\tau}=\sum_{m, s} n_{m}^{\tau s}$ is the total correlated occupancy on a site. In this work we focus on materials in which $d$ states form the correlated subspace, so at times we refer to this quantity as $N_{d}^{\tau}$ (or $N_{d}$ if there is only a single site). This double counting energy is equal to $E_{U}\left[n^{\tau s}\right]$ in the limit in which all $n_{m}^{\tau s}$ take on values of 0 or 1 .

Using this dc form, the total energy expression can be rewritten as

$$
\begin{aligned}
E_{S D F T+U}\left[\rho^{s}, n^{\tau s}\right]= & E_{S D F T}\left[\rho^{s}\right] \\
& +\frac{1}{2} U \sum_{\tau, m, s} n_{m}^{\tau s}\left(1-n_{m}^{\tau s}\right) .
\end{aligned}
$$

This form illustrates that $\mathrm{DFT}+U$ penalizes fractional occupancy of the correlated orbitals and serves to restore the derivative discontinuity of the total energy with respect to total particle number that is missing in approximate DFT [Perdew et al. (1982); Anisimov et al. (1993); Himmetoglu et al. (2014); Cococcioni and de Gironcoli (2005)].

\subsubsection{Filling and ordering energy decomposition in DFT $+U$}

Two important aspects of $\mathrm{DFT}+U$ calculations in correlated materials are (1) the total number of correlated ( $d$ or $f$ ) electrons per site and (2) the ordering of electrons within the correlated subspace based on spin (magnetism), angular momentum (orbital ordering), or site (CO). These two effects can be labeled as the "filling" and "ordering" of the correlated orbitals, respectively. Here we elucidate a decomposition of the interaction and dc energies that enables a useful decoupling of these two effects for analysis of DFT $+U$ results. 
2. Phase stability of strongly correlated electron materials within $D F T+U$

To represent the average filling on a site we define the orbital occupancy mean

$$
\mu_{\tau}=\frac{\sum_{m, s} n_{m}^{\tau s}}{N_{\text {orb }}}
$$

where $N_{\text {orb }}=\sum_{m, s} 1$ is the number of correlated spin-orbitals per site (e.g. 10 for the $d$ shell). We then rewrite the interaction and dc energies to contain only terms containing the mean $\mu_{\tau}$ or the deviation from the mean $n_{m}^{\tau s}-\mu_{\tau}$.

$$
\begin{aligned}
E_{U}-E_{d c}= & \frac{1}{2} U \sum_{\tau, m, s}\left[n_{m}^{\tau s}-\left(n_{m}^{\tau s}\right)^{2}\right] \\
= & \frac{1}{2} U \sum_{\tau, m, s}\left\{n_{m}^{\tau s}-\left[\left(n_{m}^{\tau s}-\mu_{\tau}\right)^{2}-\mu_{\tau}^{2}+2 n_{m}^{\tau s} \mu_{\tau}\right]\right\} \\
= & \frac{1}{2} U \sum_{\tau}\left[\sum_{m, s} n_{m}^{\tau s}-\sum_{m, s}\left(n_{m}^{\tau s}-\mu_{\tau}\right)^{2}+\right. \\
& \left.\mu_{\tau}^{2} \sum_{m, s} 1-2 \mu_{\tau} \sum_{m, s} n_{m}^{\tau s}\right] \\
= & \frac{1}{2} U N_{\text {orb }} \sum_{\tau}\left[\mu_{\tau}-\mu_{\tau}^{2}-\frac{\sum_{m, s}\left(n_{m}^{\tau s}-\mu_{\tau}\right)^{2}}{N_{\text {orb }}}\right] \\
= & \frac{1}{2} U N_{\text {orb }} \sum_{\tau}\left[\mu_{\tau}\left(1-\mu_{\tau}\right)-\sigma_{\tau}^{2}\right],
\end{aligned}
$$

where

$$
\sigma_{\tau}=\sqrt{\frac{\sum_{m, s}\left(n_{m}^{\tau s}-\mu_{\tau}\right)^{2}}{N_{o r b}}}
$$

is the standard deviation of the occupancies for site $\tau$. We call $\mu_{\tau}\left(1-\mu_{\tau}\right)$ the filling factor and $\sigma_{\tau}^{2}$ the ordering factor, and at times in our discussion of results we drop the $\tau$ subscript on $\mu_{\tau}$ and $\sigma_{\tau}$ for convenience. $E_{U}-E_{d c}$ is thus rewritten as $E_{\text {fill }}+E_{\text {ord }}$ with:

$$
E_{\text {fill }}=\frac{1}{2} U N_{\text {orb }} \sum_{\tau} \mu_{\tau}\left(1-\mu_{\tau}\right)
$$


and

$$
E_{\text {ord }}=-\frac{1}{2} U N_{\text {orb }} \sum_{\tau} \sigma_{\tau}^{2}
$$

These terms encapsulate the two ways in which a system can avoid paying the Coulomb energetic penalty $U$. From the filling term, the system can minimize $\mu_{\tau}\left(1-\mu_{\tau}\right)$ by moving towards completely empty $\left(\mu_{\tau}=0\right)$ or completely filled $\left(\mu_{\tau}=1\right)$ correlated shells on average. From the ordering term, the system can maximize $\sigma_{\tau}^{2}$ by enhancing the spread in $n_{m}^{\tau s}$ via some type of ordering. Note that $n_{m}^{\tau s}$ values for nominally-unoccupied orbitals still contribute to $\sigma_{\tau}$, so this quantity is distinct from other measures of orbital ordering are determined solely by nominally occupied orbitals.

In this work we are primarily interested in the formation energy, which is defined as

$$
F E(x)=E(x)-[(1-x) E(0)+x E(1)]
$$

where $E(x)$ is the cohesive energy of a system with intercalant concentration $x$. The formation energy indicates whether a species of intermediate $x$ has a higher or lower cohesive energy than the corresponding linear combination of those of the endmembers. Therefore, only energy terms beyond linear order in $x$ contribute. The formation energy dictates the stability of such a species in the limit of low temperature $(T \rightarrow 0)$; it is stable if negative and unstable if positive. One can separately construct the formation energy contributions stemming from $E_{D F T}, E_{U}-E_{d c}, E_{\text {fill }}, E_{\text {ord }}$ using expressions analogous expressions to Eq. 2.11, allowing one to understand the contribution of each term to the formation energy. Note that $F E_{\text {fill }}$ will be negative if $\mu(1-\mu)$ is lower than the endmember linear combination, while $F E_{\text {ord }}$ will be negative if $\sigma^{2}$ is higher than the endmember linear combination due to the negative sign in the definition of $E_{\text {ord }}$ in Eq. 2.10. All formation energies reported in this work are normalized per formula unit (f.u.). We also compute the average intercalation voltage $V$ via $e V=E(\mathrm{Li})+E(0)-E(1)$, where $e$ is the elementary charge, $E(\mathrm{Li})$ is the cohesive energy of body-centered-cubic Li, and the energies are normalized to the number 
of f.u [Aydinol et al. (1997)].

\subsection{Computational Details}

$\mathrm{DFT}+U$ calculations based on the spin-dependent generalized gradient approximation [Perdew et al. (1996)], and the rotationally-invariant Hubbard $U$ interaction [Liechtenstein et al. (1995)] with $J$ set to 0, and FLL dc are performed using the Vienna ab initio simulation package (VASP) [Kresse and Hafner (1994, 1993); Kresse and Furthmüller (1996b,a)]. The projector augmented wave method [Blöchl (1994); Kresse and Joubert (1999)] is employed and the single-particle equations are solved with a plane-wave basis set with a kinetic energy cutoff of $500 \mathrm{eV}$. We use a $9 \times 9 \times 9(6 \times 7 \times 8) k$-point grid for the rhombohedral (orthorhombic) primitive unit cell of $\mathrm{Li}_{x} \mathrm{CoO}_{2}\left(\mathrm{Li}_{x} \mathrm{FePO}_{4}\right.$ and $\left.\mathrm{Li}_{x} \mathrm{CoPO}_{4}\right)$ and $k$-point grids with approximately the same $k$-point density for supercell calculations. The bulk lithium calculation is performed using a $19 \times 19 \times 19 k$-point grid. For structural relaxations in metals we employ the first-order Methfessel-Paxton method [Methfessel and Paxton (1989)] with a $50 \mathrm{meV}$ smearing and for all other calculations the tetrahedron method with Blöchl corrections [Blöchl et al. (1994)] is used. The ionic forces, stress tensor components, and total energy are converged to $0.01 \mathrm{eV} / \AA, 10^{-3} \mathrm{GPa}$, and $10^{-6} \mathrm{eV}$, respectively.

The disordered (solid solution) phase of $\mathrm{Li}_{1 / 2} \mathrm{CoO}_{2}$ is modeled via the special quasirandom structure (SQS) approach [Zunger et al. (1990)] using the Alloy Theoretic Automated Toolkit (АТАT) [van de Walle et al. (2002); van de Walle (2009)] using point, pair, triplet, and quadruplet clusters. Candidate structures are generated based on the correlation functions of clusters with a maximum inter-site distance up to the in-plane 2nd nearest neighbor and out-of-plane 1st nearest neighbor distance. To evaluate the structures, we consider the minimal values of the following figure of merit: the root-sum-square of the differences between the cluster correlation functions and those of the random structure. In this evaluation, the 
2. Phase stability of strongly correlated electron materials within $D F T+U$

correlation functions of clusters containing a maximum inter-site distance up to the in-plane 5th nearest neighbor and inter-plane 6th nearest neighbor are taken into account.

\subsection{Results and Discussion}

\subsubsection{Endmember electronic structure within DFT}

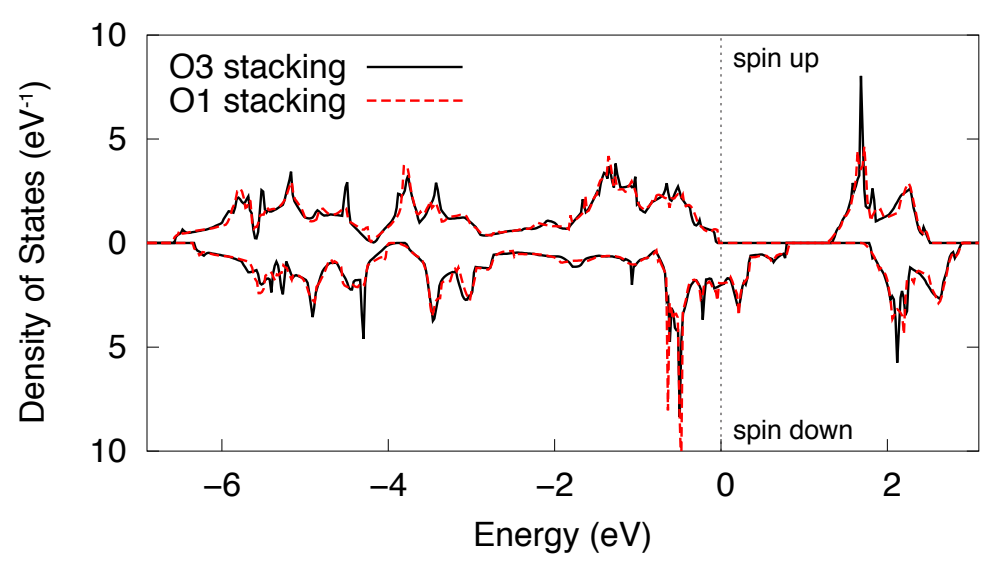

Figure 2.2: Electronic density of states for $\mathrm{CoO}_{2}$ in the $\mathrm{O} 3$ structure (solid black lines) and O1 structures (dashed red lines) within DFT, indicating the different layer stacking has a very small effect on the density of states. The thin dashed black line indicates the Fermi energy.

We begin by reviewing the electronic structure of the endmembers of $\mathrm{Li}_{x} \mathrm{CoO}_{2}$ and $\mathrm{Li}_{x} \mathrm{FePO}_{4}$ within DFT, comparing with the latest experimental understanding of these materials. $\mathrm{LiCoO}_{2}$ crystallizes in the layered structure illustrated in Fig. 2.1(a) consisting of layers of edge-sharing $\mathrm{CoO}_{6}$ octahedra and layers of Li. It has a rhombohedral primitive unit cell with the $R \overline{3} m$ space group and A-B-C (O3) oxygen stacking [Johnston et al. (1958); Orman and Wiseman (1984)]. $\mathrm{CoO}_{2}$ has a very similar structure with a hexagonal unit cell and A-B (O1) oxygen stacking in the $P \overline{3} m 1$ space group [Amatucci et al. (1996)]. Here 

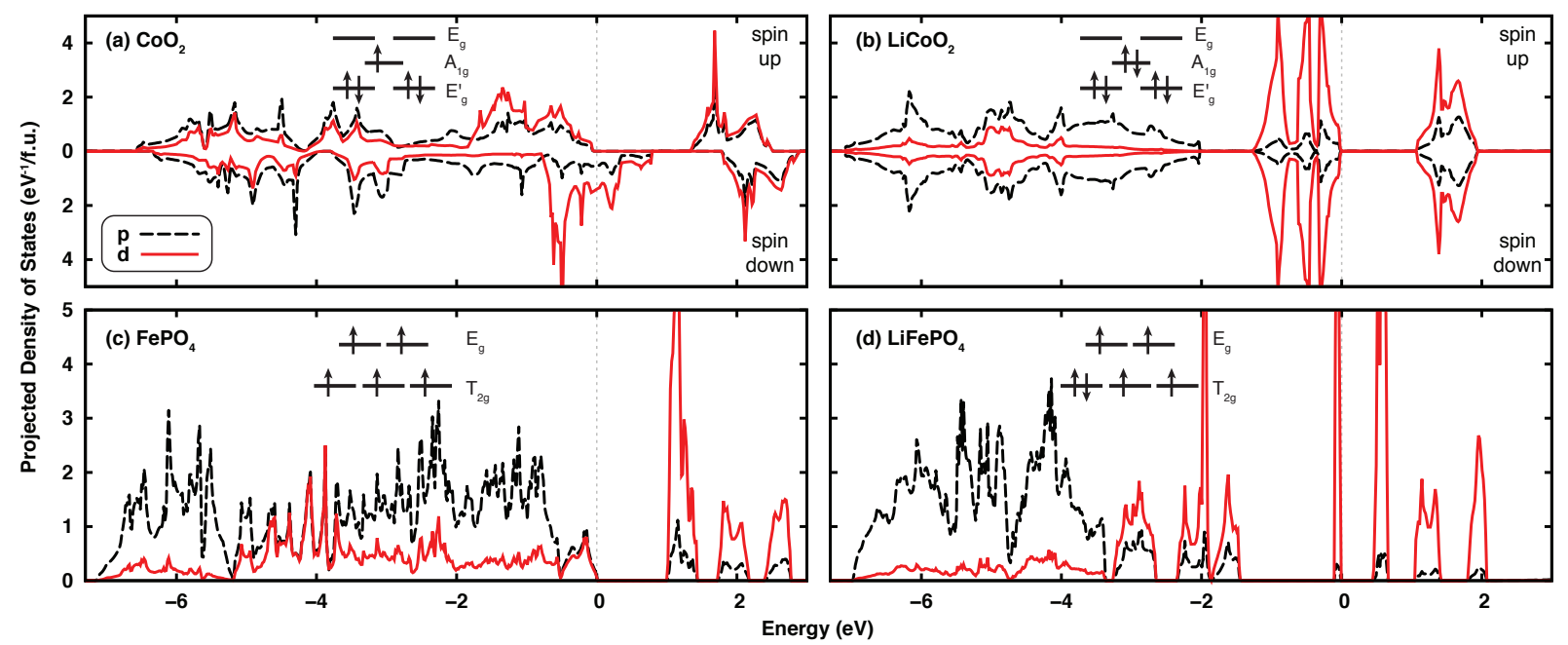

Figure 2.3: Projected $p$ and $d$ density of states for (a) $\mathrm{CoO}_{2}$, (b) $\mathrm{LiCoO}_{2}$, (c), $\mathrm{FePO}_{4}$, and (d) $\mathrm{LiFePO}_{4}$ within DFT. For antiferromagnetic $\mathrm{FePO}_{4}$ and $\mathrm{LiFePO}_{4}$ only a single spin channel is shown. The dashed gray lines indicate the valence band maximum for insulators and the Fermi energy for metals. Insets are the nominal transition metal $3 d$ orbital fillings from crystal field theory.

for convenience we consider $\mathrm{CoO}_{2}$ in the $\mathrm{O} 3$ structure, which has a very similar electronic structure to that of the O1 structure within DFT as shown in Fig. 2.2.

In $\mathrm{Li}_{x} \mathrm{CoO}_{2}$ the octahedral coordination of $\mathrm{Co}$ is slightly distorted due to the ability of the oxygens to relax in the out-of-plane direction, resulting in a symmetry lowering with $T_{2 g} \rightarrow A_{1 g}+E_{g}^{\prime}$. Nominally $\mathrm{LiCoO}_{2}$ is in the $d^{6}$ configuration with filled $E_{g}^{\prime}$ and $A_{1 g}$ levels, while $\mathrm{CoO}_{2}$ has one fewer electron $\left(d^{5}\right)$.

The $p$ and $d$ projection of the electronic density of states within DFT for $\mathrm{CoO}_{2}$ and $\mathrm{LiCoO}_{2}$ are shown in Fig. 2.3(a) and 2.3(b), respectively. $\mathrm{LiCoO}_{2}$ is found to be a band insulator in agreement with experiments [van Elp et al. (1991); Ménétrier et al. (1999, 2008)]. The computed band gap of $1.1 \mathrm{eV}$ underestimates the experimental value of $2.7 \mathrm{eV}$ as is typical for DFT [van Elp et al. (1991)]. The occupancies of $A_{1 g}, E_{g}^{\prime}$ and $E_{g}$ are 0.94, 0.95, and 0.41 for $\mathrm{LiCoO}_{2}$, demonstrating that hybridization between $\mathrm{Co} d$ and $\mathrm{O} p$ states leads to appreciable occupation of the nominally-unoccupied $E_{g}$ states. $\mathrm{CoO}_{2}$ has a ferromagnetic 
low-spin metallic ground state with a Co magnetic moment of $0.8 \mu_{B}$. In experiments on $\mathrm{CoO}_{2}$, there is evidence for Fermi liquid behavior and Pauli paramagnetism without any long-range magnetic ordering [de Vaulx et al. (2007); Motohashi et al. (2007); Kawasaki et al. (2009)]. For $\mathrm{CoO}_{2}$ the occupation of $A_{1 g}, E_{g}^{\prime}$ and $E_{g}$ are 0.96 (0.67), 0.96 (0.76), and 0.54 (0.52) for spin up (down) electrons, showing the significant degree of covalency in this system.

$\mathrm{LiFePO}_{4}$ and $\mathrm{FePO}_{4}$ both take on the olivine structure, which has an orthorhombic primitive unit cell containing 4 formula units in the Pnma space group [Santoro and Newnham (1967); Rousse et al. (2003)]. The structure consists of corner-sharing $\mathrm{FeO}_{6}$ octahedra layers connected via $\mathrm{PO}_{4}$ tetrahedra as shown in Fig. 2.1(b). For $\mathrm{LiFePO}_{4}$ there are onedimensional chains of $\mathrm{Li}$ ions.

$\mathrm{Li}_{x} \mathrm{FePO}_{4}$ has significantly distorted $\mathrm{FeO}_{6}$ octahedra, though for convenience we still crudely group the $d$ orbitals as $T_{2 g}$ and $E_{g}$. Experimentally $\mathrm{LiFePO}_{4}$ and $\mathrm{FePO}_{4}$ are high-spin antiferromagnetic (AFM) insulators with Néel temperatures of 52 and $125 \mathrm{~K}$, respectively [Santoro and Newnham (1967); Rousse et al. (2003)]. While $\mathrm{FePO}_{4}\left(d^{5}\right)$ nominally has all the $d$ orbitals on a given site singly occupied with aligned spins (i.e., $S=5 / 2$ ), for $\mathrm{LiFePO}_{4}$ $\left(d^{6}\right)$ there is one additional minority-spin electron in the $T_{2 g}$ manifold [Tang and Holzwarth (2003); Shi et al. (2005)]. Fe linked via corner-sharing octahedra in the same layer have anti-aligned magnetic moments, while those laterally adjacent in different layers linked via $\mathrm{PO}_{4}$ have aligned magnetic moments [Santoro and Newnham (1967); Rousse et al. (2003)].

The projected density of states for the olivine endmembers are shown in Fig. 2.3(c) and 2.3(d). Due to the antiferromagnetism, both spin channels are identical so only one is shown. $\mathrm{FePO}_{4}$ can be viewed as a charge transfer type insulator since the gap is $p-d$ in nature, whereas in $\mathrm{LiFePO}_{4} d$ states form both the valence and conduction bands and the electronic bandwidths near the Fermi energy are extremely narrow (as little as $0.1 \mathrm{eV}$ ). Although $\mathrm{LiFePO}_{4}$ has an even number of electrons, the local Coulomb interaction can play a strong role in developing or enhancing the insulating behavior. Within DFT, the band 


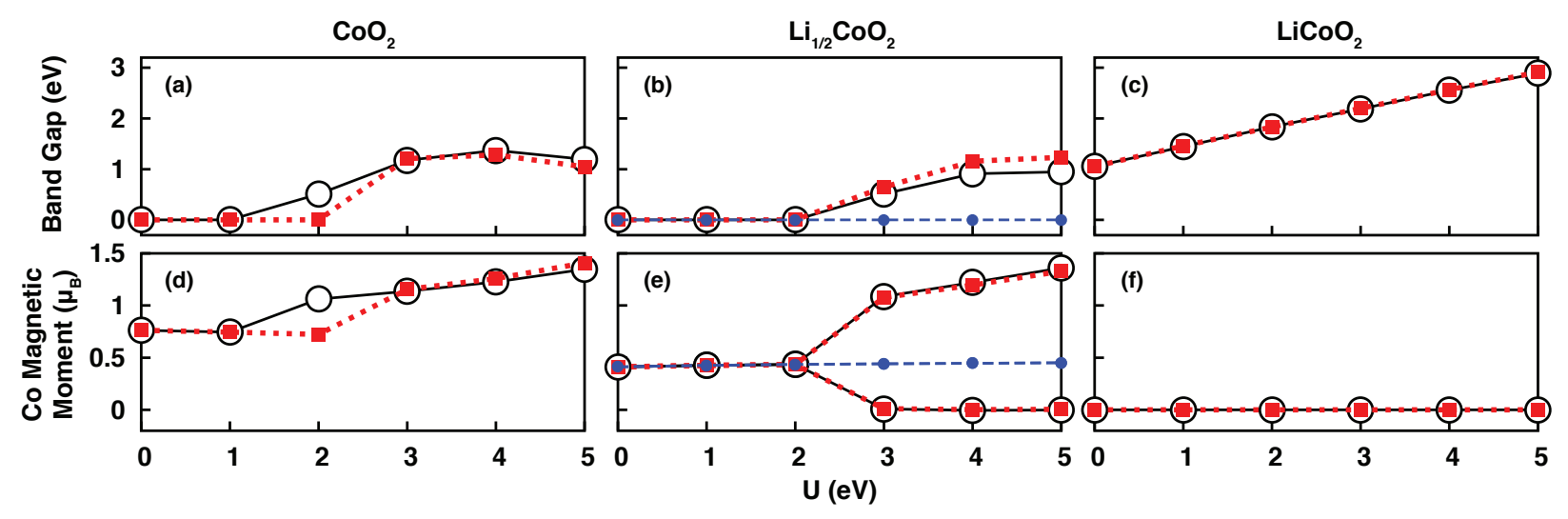

Figure 2.4: Electronic band gap as a function of $U$ for (a) $\mathrm{CoO}_{2}$, (b) $\mathrm{Li}_{1 / 2} \mathrm{CoO}_{2}$, and (c) $\mathrm{LiCoO}_{2}$ with relaxations (filled red squares) and with frozen $U=0$ structures (large open black circles). The corresponding plots of Co magnetic moment are shown in panels (d)-(f). For panels (b) and (e), the additional small filled blue circles correspond to calculations with frozen $U=0$ structures and CO suppressed.

gaps of $\mathrm{FePO}_{4}$ and $\mathrm{LiFePO}_{4}$ are $1.0 \mathrm{eV}$ and $0.4 \mathrm{eV}$, respectively. These values are brought much closer to agreement with the experimental band gaps of $1.9 \mathrm{eV}$ [Zaghib et al. (2007)] and $3.8 \mathrm{eV}$ [Zhou et al. (2004b); Zaghib et al. (2007)] using the DFT $+U$ approach [Zhou et al. (2004b); Seo et al. (2015)].

\subsubsection{Impact of $U$ on electronic structure of $\mathrm{Li}_{x} \mathrm{CoO}_{2}$}

The on-site interaction $U$ has been computed as 4.9 and $5.4 \mathrm{eV}$ for $\mathrm{LiCoO}_{2}$ and $\mathrm{CoO}_{2}$, respectively [Zhou et al. (2004a)]. The $U$-dependence of the band gap and Co magnetic moment for $\mathrm{Li}_{x} \mathrm{CoO}_{2}$ are shown in Fig. 2.4. The band insulator $\mathrm{LiCoO}_{2}$ has no magnetic moment and its band gap increases roughly linearly with $U$ from $1.1 \mathrm{eV}$ at $U=0$ to $2.9 \mathrm{eV}$ for $U=5 \mathrm{eV}$. Structural relaxations with $U$ have little impact on the electronic structure. Note that we do not explore $U>5 \mathrm{eV}$ since in this regime we find $\mathrm{DFT}+U$ predicts a high-spin state for $\mathrm{LiCoO}_{2}$ [Andriyevsky et al. (2014)] in contradiction with experimental observation [Ménétrier et al. (1999, 2008)]. 
2. Phase stability of strongly correlated electron materials within $D F T+U$

$\mathrm{CoO}_{2}$ is semimetallic at lower values of $U$ with the $A_{1 g}$ and $E_{g}^{\prime}$ states both partially occupied. Beyond $U=1 \mathrm{eV}$ (or $U=2 \mathrm{eV}$ when including structural relaxations), an orbital ordering occurs in which $E_{g}^{\prime}$ completely fills and $A_{1 g}$ becomes a nominally half-filled $S=1 / 2$ state. This opens up a band gap of $0.5-1.2 \mathrm{eV}$ and increases the Co magnetic moment to 1-1-1.4 $\mu_{B}$, as compared to $0.7-0.8 \mu_{B}$ for lower $U$.

The lowest-energy structure of $\mathrm{Li}_{1 / 2} \mathrm{CoO}_{2}$ has an in-plane ordering of $\mathrm{Li}$ and vacancies corresponding to the unit cell shown in Fig. 2.1(a) with the Li1 ion removed [Reimers and Dahn (1992); Van der Ven et al. (1998); Wolverton and Zunger (1998)]. Experimental studies suggest $\mathrm{Li}_{1 / 2} \mathrm{CoO}_{2}$ is a paramagnetic metal with small Co magnetic moments of around 0.25$0.35 \mu_{B}$ [Motohashi et al. (2009); Miyoshi et al. (2010); Ou-Yang et al. (2012)]. Within our calculations $\mathrm{Li}_{1 / 2} \mathrm{CoO}_{2}$ is a ferromagnetic metal for $U \leq 2 \mathrm{eV}$ with equal Co magnetic moments of $0.4 \mu_{B}$. For larger $U$ values a new ground state with $\mathrm{CO}$ emerges in which the first site takes on a $\mathrm{CoO}_{2}$-like configuration with a moment of 1.1-1.4 $\mu_{B}$ and the second takes on a $\mathrm{LiCoO}_{2}$-like configuration with no moment. $\mathrm{CO}$ opens an electronic band gap that increases with $U$ of $0.6-1.2 \mathrm{eV}(0.5-0.9 \mathrm{eV}$ without structural relaxations). Ignoring structural relaxations, the metallic state without CO is metastable and the Co magnetic moments are $0.4-0.5 \mu_{B}$ and remain roughly constant for all values of $U$ considered.

\subsubsection{Tendency for charge ordering in DFT $+U$}

To understand the tendency for $\mathrm{CO}$ in $\mathrm{DFT}+U$, we investigated whether its origin is the interaction $(U)$ term or the de term. To do so, we implemented a modified DFT $+U$ approach in which the single-particle potential and total energy contributions stemming from the interaction term or the de term are averaged over correlated sites. We call this site-averaged interaction and site-averaged dc, respectively.

Figure 2.5 illustrates the results of this computational experiment for ordered $\mathrm{Li}_{1 / 2} \mathrm{CoO}_{2}$ using the frozen $U=0$ structure. With site-averaged dc (black squares) we still find a CO 

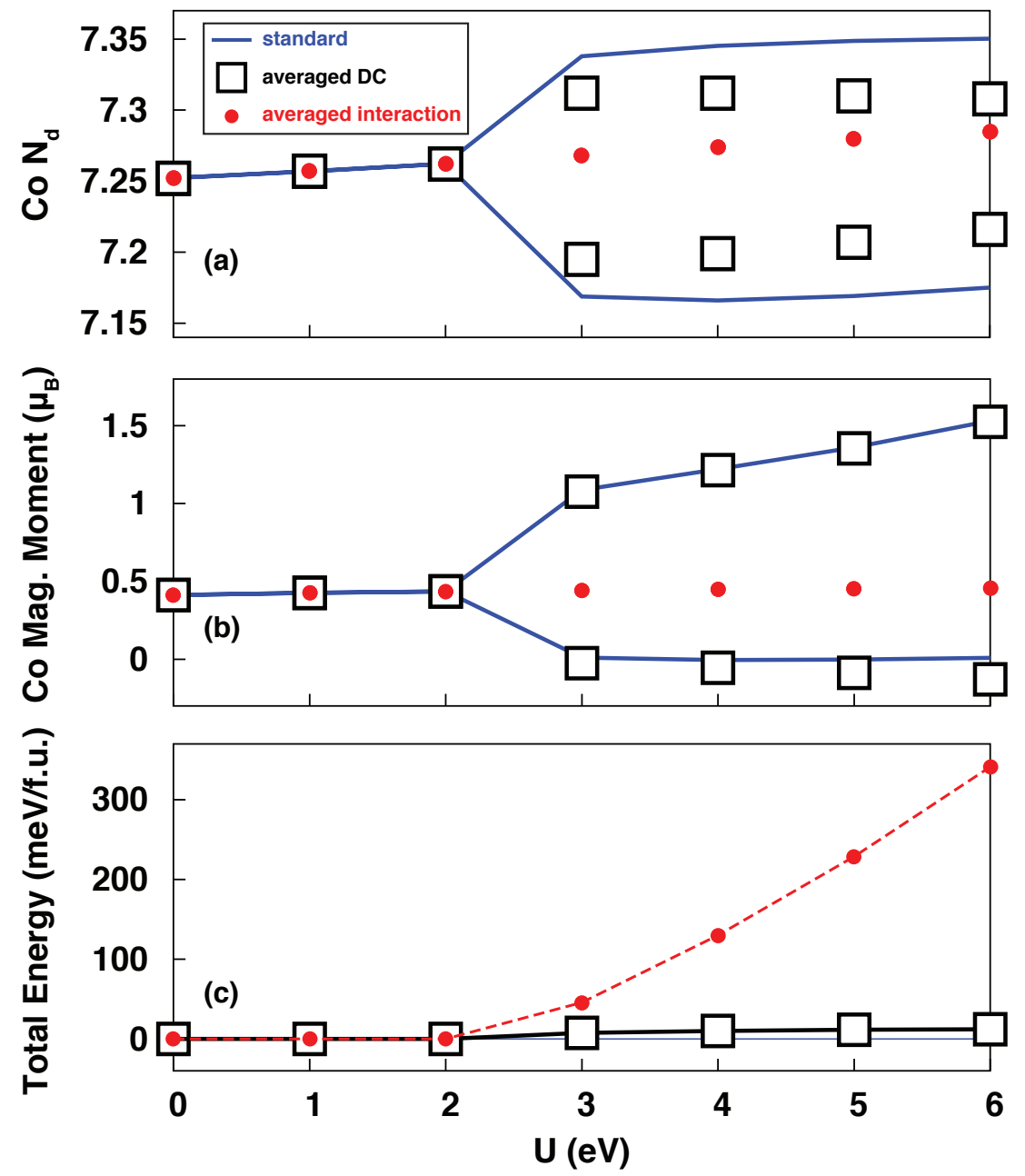

Figure 2.5: (a) Co $\mathrm{N}_{d}$, (b) Co magnetic moments, and (c) total energy for $\mathrm{Li}_{1 / 2} \mathrm{CoO}_{2}$ with the frozen $U=0 \mathrm{eV}$ structure for standard $\mathrm{DFT}+U$ (blue lines) as well as with the interaction (filled red circles) or double counting (open black squares) terms averaged over correlated sites. Total energies in panel (c) are with respect to those of the standard $\mathrm{DFT}+U$. 
transition, for $U>2 \mathrm{eV}$. The magnitude of the CO in terms of $N_{d}$ is slightly reduced, but the deviations in Co magnetic moment are the same or even more substantial than the standard $\mathrm{DFT}+U$ results (blue lines). The total energy shown in panel (c) for this case is only slightly (on the order of $10 \mathrm{meV} /$ f.u.) higher than in the case of standard DFT $+U$, which indicates that the dc energetics are not very much lowered via CO. With site-averaged interaction (red circles), however, no CO can be obtained and the total energy is massively penalized as $U$ increases (by hundreds of $\mathrm{meV} / \mathrm{f} . u$.). Therefore, we conclude that it is the interaction term and not the de term that is responsible for the $\mathrm{CO}$ in $\mathrm{DFT}+U$. This suggests that more accurate solutions to the interaction problem such as dynamical mean-field theory have potential to solve this issue of artificial CO.

\subsubsection{Impact of $U$ on phase stability of $\mathrm{Li}_{x} \mathrm{CoO}_{2}$}

We first consider the formation energy of $\mathrm{Li}_{1 / 2} \mathrm{CoO}_{2}$ in the frozen $U=0$ structure (i.e., no structural relaxations when imposing $U$ ) and without allowing $\mathrm{CO}$, in order to purely see the effects of $U$ in the absence of $\mathrm{CO}$ and lattice distortions. For the experimental Li ordering in $\mathrm{Li}_{1 / 2} \mathrm{CoO}_{2}$, the two $\mathrm{Co}$ atoms in the unit cell are equivalent by point symmetry. Therefore $\mathrm{CO}$ is a spontaneously broken symmetry, which enables one to precisely investigate various observables with and without CO. We will return to the effects of both CO and structural relaxations after thoroughly explaining the role of $U$ in their absence.

As shown in Fig. 2.6(a), $\mathrm{Li}_{1 / 2} \mathrm{CoO}_{2}$ is phase stable with a total formation energy of -217 meV for $U=0$. The formation energy increases monotonically with $U$ and for $U>3 \mathrm{eV}$ it becomes positive, corresponding to a prediction of phase separation. This indicates that the trend of a destabilization of compounds of intermediate $x$ found previously in $\mathrm{Li}_{x} \mathrm{FePO}_{4}$ [Zhou et al. (2004c)] also occurs for $\mathrm{Li}_{x} \mathrm{CoO}_{2}$. Furthermore, it demonstrates that such a trend is found even in the absence of CO.

To illustrate the origin of this behavior, we also examine the DFT and $U$-dc components 

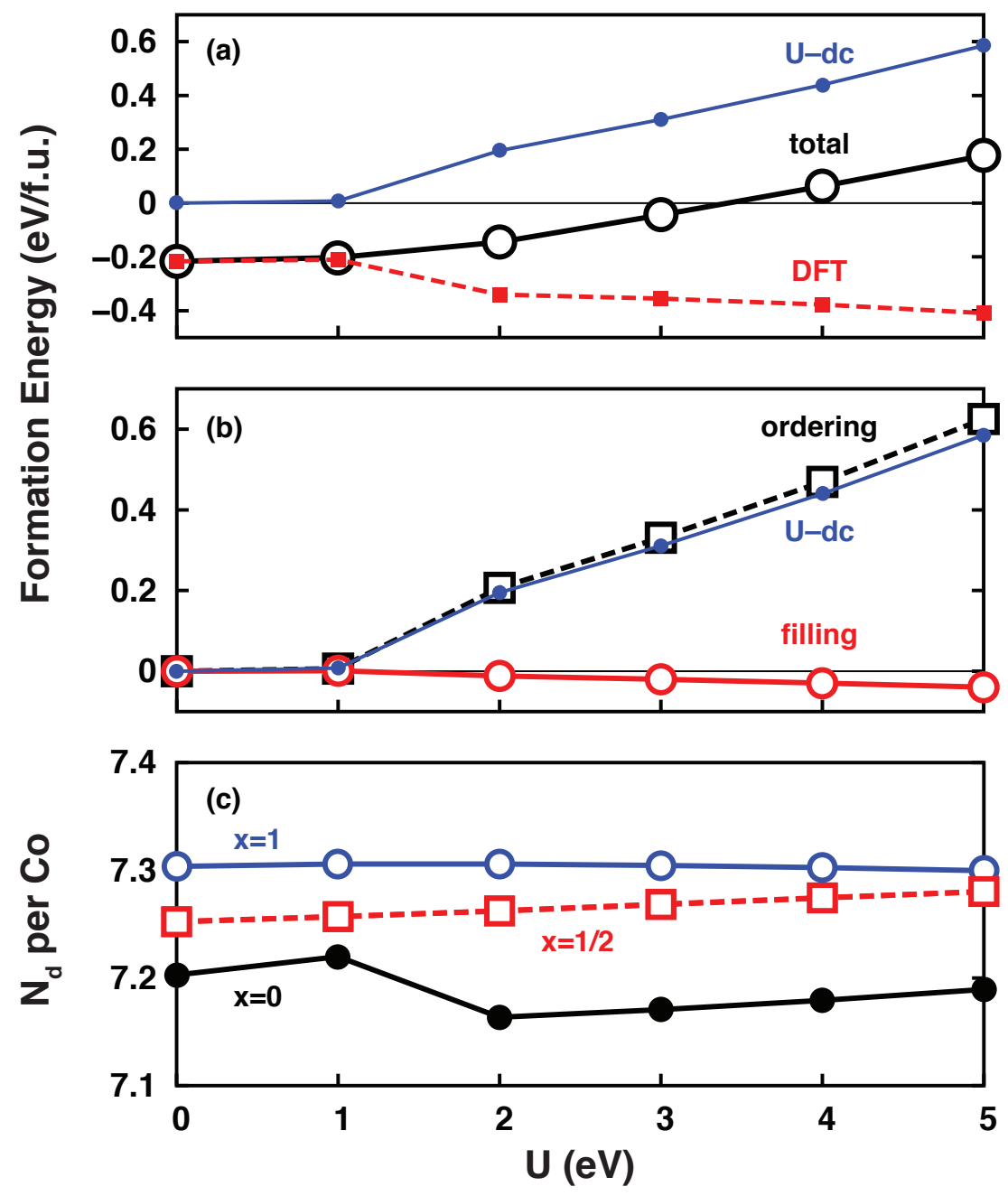

Figure 2.6: (a) Total $\mathrm{Li}_{1 / 2} \mathrm{CoO}_{2}$ formation energy (open black circles) and its DFT (filled red squares) and $U$-dc (filled blue circles) components as a function of $U$ (b) $\mathrm{Li}_{1 / 2} \mathrm{CoO}_{2} U$-dc (filled blue circles) formation energy component and its orbital filling (open red circles) and orbital ordering (open black squares) components as a function of $U$ (c) Number of $d$ electrons per $\mathrm{Co}$ as a function of $U$ for $\mathrm{CoO}_{2}$ (filled black circles), $\mathrm{Li}_{1 / 2} \mathrm{CoO}_{2}$ (open red squares), and $\mathrm{LiCoO}_{2}$ (open blue circles). All data correspond to the case of frozen $U=0$ structures and $\mathrm{CO}$ suppressed in $\mathrm{Li}_{1 / 2} \mathrm{CoO}_{2}$. 
of the total formation energy in Fig. 2.6(a). For $U=1 \mathrm{eV}$ the $U$-dc component is negligible $(<0.01 \mathrm{eV})$ and the slightly less negative value of formation energy $(-203 \mathrm{meV})$ stems from the DFT component. Compared to the $U=0$ values, for $U=1 \mathrm{eV}$ the DFT energy increases by $17 \mathrm{meV}$ for $\mathrm{Li}_{1} / 2 \mathrm{CoO}_{2}$ compared to only $7 \mathrm{meV}$ for $\mathrm{CoO}_{2}$ and $14 \mathrm{meV}$ for $\mathrm{LiCoO}_{2}$. For larger $U$ the $U$-dc component is strongly positive and increases roughly linearly with $U$ at a rate of around $130 \mathrm{meV}$ per eV, leading to a more rapid increase in total formation energy. The DFT component has the opposite trend of becoming more negative with $U$, largely since the DFT energy of $\mathrm{CoO}_{2}$ is strongly penalized by the orbital ordering, but the overall effect on the formation energy is smaller with changes of around $15-30 \mathrm{meV}$ per eV. Therefore, it is the $U$-dc component that is responsible for the destabilization of $\mathrm{Li}_{1 / 2} \mathrm{CoO}_{2}$.

The number of $d$ electrons per Co site $\left(N_{d}\right)$ is plotted for $x=0, x=1 / 2$, and $x=1$ as a function of $U$ in Fig. 2.6(c). We note that the difference in $N_{d}$ between $\mathrm{CoO}_{2}$ and $\mathrm{LiCoO}_{2}$ is only around 0.1 even though $\mathrm{Li}$ is nominally donating a full electron, which is due to the $p-d$ rehybridization effect in $\mathrm{Li}_{x} \mathrm{CoO}_{2}$ [Wolverton and Zunger (1998); Marianetti et al. (2004)]. While the behavior of $N_{d}$ is roughly constant at 7.3 for $\mathrm{LiCoO}_{2}$ and smoothly increasing for $\mathrm{Li}_{1 / 2} \mathrm{CoO}_{2}$ between 7.25 and 7.28, there is discontinuous behavior for $\mathrm{CoO}_{2}$ in which $N_{d}$ drops from 7.22 to 7.16 from $U=1$ to $U=2 \mathrm{eV}$ corresponding to the orbital ordering. This change in electronic structure in the $x=0$ endmember is responsible for the change in behavior in the $U$-dc formation energy contribution.

Since the dc term in Eq. 2.4 is a negative quadratic function, one might expect that the $\mathrm{dc}$ is responsible for the trend towards phase separation. This simple line of reasoning immediately becomes more complicated given that $N_{d}$ is a nonlinear function of $x$ as demonstrated in Fig. 2.6(c), and a careful analysis in Sec. 2.4 .8 shows that it is not the de that drives phase separation. Alternatively, we proceed to understand the contributions of both the $E_{U}$ and $E_{d c}$ terms simultaneously in a different framework using the energy decomposition described in Sec. 2.2.2. In Fig. 2.6(b) we break down the $U$-dc formation energy contribution into the filling and ordering contributions. Remarkably, the magnitude of the filling contribution 
contributes negligibly, only being at most tens of meV in magnitude, whereas essentially all of the $U$-dc formation energy comes from the ordering term. Therefore, it is the ordering rather than the filling of the correlated $d$ orbitals that drives phase separation.

The individual filling factor $\mu(1-\mu)$ and ordering factor $\sigma^{2}$ as a function of Li concentration for different $U$ are shown for this case in Fig. 2.7(a) and 2.7(d), respectively. We note that based on nominal electron counting $\mu(1-\mu)$ will be 0.25 for $\mathrm{Co}^{4+}$ and 0.24 for $\mathrm{Co}^{3+}$. Consistent with this expectation, we observe that the filling factor is highest for $x=0$ and lowest for $x=1$. The actual values for $\mathrm{Li}_{x} \mathrm{CoO}_{2}$ are lower in magnitude (around 0.2) due to the substantial covalent nature of the bonding, in particular the hybridization of $\mathrm{O} p$ states with Co $E_{g}$ states. Although this filling factor magnitude is around half an order of magnitude higher than that of the ordering factor, the relative deviations of the $x=1 / 2$ value compared to the average of the $x=0$ and $x=1$ values are tiny (around $10^{-3}$ electrons). This is why filling term leads to a negligible contribution to the formation energy.

The values of $\sigma^{2}$ increase with $U$ for all $x$ in agreement with the expectation that the $U$ and dc parts of the total energy functional will penalize fractional orbital occupancy (i.e., $0<n_{m}^{\tau s}<1$ ). For $U \leq 1 \mathrm{eV} \sigma^{2}$ is nearly linear in $x$, thus leading to no appreciable formation energy contribution. However, once $\mathrm{CoO}_{2}$ undergoes the orbital ordering, its value of $\sigma^{2}$ significantly increases from 0.03 to 0.08 . After this phase transition, $\sigma$ for $\mathrm{Li}_{1 / 2} \mathrm{CoO}_{2}$ is substantially lower than the average of those of $\mathrm{CoO}_{2}$ and $\mathrm{LiCoO}_{2}$. For example, for $U=2$ $\mathrm{eV} \sigma$ is 0.277 for $\mathrm{CoO}_{2}$ and 0.282 for $\mathrm{LiCoO}_{2}$ but only 0.239 for $\mathrm{Li}_{1 / 2} \mathrm{CoO}_{2}$. This lower-thanaverage $\sigma^{2}$ is translated to a positive formation energy contribution via the negative sign in prefactor of Eq. 2.10.

The fundamental behavior we find is that $U$ drives phase separation via enhanced ordering of the correlated orbitals for the endmembers relative to the species with intermediate $\mathrm{Li}$ concentration. For $U \leq 1 \mathrm{eV} \sigma^{2}$ is much smaller for $x=0$ than $x=1 ; \mathrm{CoO}_{2}$ has a smaller range of $n_{m}^{\tau s}$ due to its semimetallic behavior and enhanced hybridization with $\mathrm{O} p$ states. Once it has orbitally ordered at $U=2 \mathrm{eV}$ and the hole in the $T_{2 g}$ manifold is localized in 


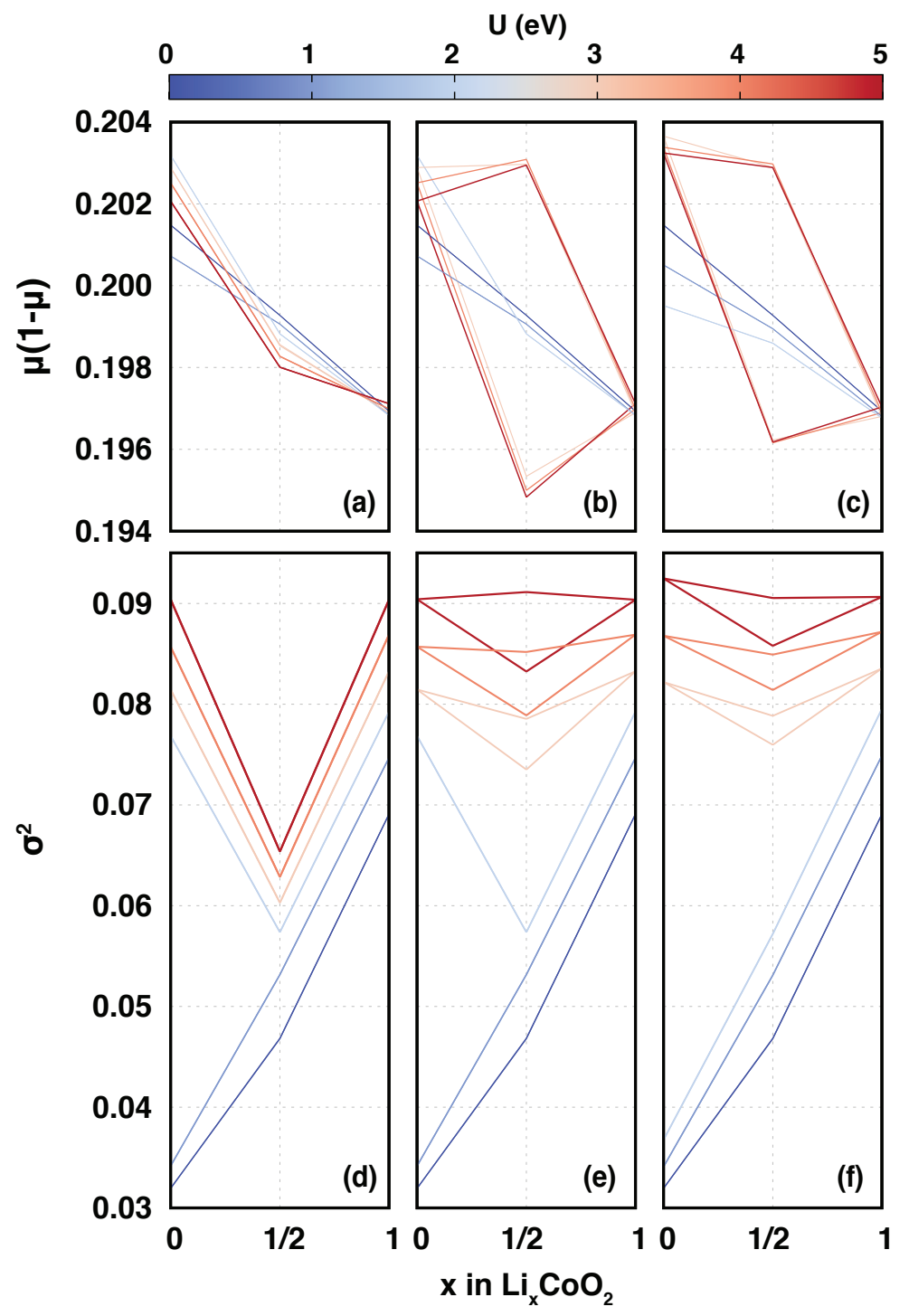

Figure 2.7: Filling factor $\mu(1-\mu)$ as a function of $x$ for different $U$ in $\operatorname{Li}_{x} \mathrm{CoO}_{2}$ for (a) frozen $U=0$ structures and CO suppressed, (b) frozen $U=0$ structures and $\mathrm{CO}$ allowed, and (c) relaxed structures. Panels (d)-(f) show the corresponding plots for the ordering factor $\sigma^{2}$. The two lines per $U$ in some plots correspond to the two distinct $\mathrm{Co}$ sites in $\mathrm{Li}_{1 / 2} \mathrm{CoO}_{2}$. The line thickness increases for increasing values of $U$. 

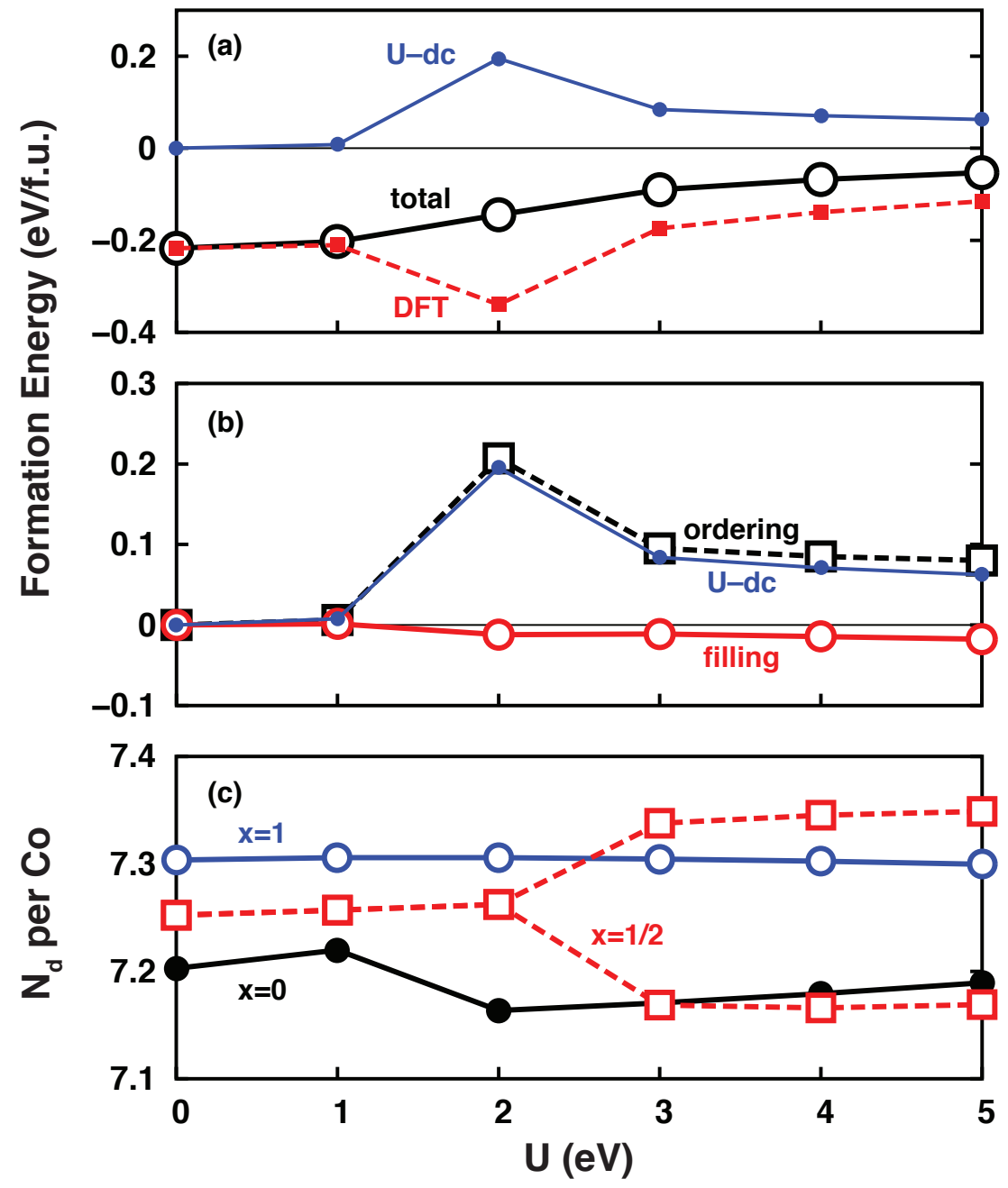

Figure 2.8: (a) Total $\mathrm{Li}_{1 / 2} \mathrm{CoO}_{2}$ formation energy (open black circles) and its DFT (filled red squares) and $U$-dc (filled blue circles) components as a function of $U$ (b) $\mathrm{Li}_{1 / 2} \mathrm{CoO}_{2} U$-dc (filled blue circles) formation energy component and its orbital filling (open red circles) and orbital ordering (open black squares) components as a function of $U$ (c) Number of $d$ electrons per Co as a function of $U$ for $\mathrm{CoO}_{2}$ (filled black circles), $\mathrm{Li}_{1 / 2} \mathrm{CoO}_{2}$ (open red squares), and $\mathrm{LiCoO}_{2}$ (open blue circles). All data correspond to the case of frozen $U=0$ structures and $\mathrm{CO}$ allowed and the two lines in panel (c) for $\mathrm{Li}_{1 / 2} \mathrm{CoO}_{2}$ correspond to the two distinct Co sites. 
the minority-spin $A_{1 g}$ state, however, $\mathrm{CoO}_{2}$ has 5 very occupied $d$ orbitals $\left(n_{m}^{\tau s} \geq 0.96\right)$ and 5 much less occupied orbitals ( $n_{m}^{\tau s}$ of around 0.15 for minority-spin $A_{1 g}$ and higher values of $0.49-0.59$ for $E_{g}$ due to $p-d$ hybridization). $\mathrm{LiCoO}_{2}$ is a band insulator, so there is no abrupt change in $\sigma^{2}$ as a function of $U$. In terms of $\sigma^{2}, \mathrm{LiCoO}_{2}$ behaves very similarly to $\mathrm{CoO}_{2}$ in the regime of $U$ in which $\mathrm{CoO}_{2}$ is orbitally ordered. In $\mathrm{LiCoO}_{2}$ there are 6 very occupied $d$ orbitals ( $n_{m}^{\tau s} \approx 0.96$ for $A_{1 g}$ and $E_{g}^{\prime}$ states) and 4 much less occupied orbitals $\left(n_{m}^{\tau s}=0.39\right.$ for $E_{g}$ states). This gives a large spread $(\sigma)$ in orbital occupancies for $\mathrm{LiCoO}_{2}$ in addition to $\mathrm{CoO}_{2}$. In contrast, for $\mathrm{Li}_{1 / 2} \mathrm{CoO}_{2}$ we have a metallic state with a nominally half-filled minority-spin $A_{1 g}$ level $\left(n_{m}^{\tau s} \approx 0.61\right)$ and thus smaller $\sigma^{2}$. This lower-than-average $\sigma^{2}$ for $\mathrm{Li}_{1 / 2} \mathrm{CoO}_{2}$ is what results in a positive contribution to the formation energy from the $U$-dc energetics.

This same type of behavior is preserved even when we now allow $\mathrm{CO}$ in $\mathrm{Li}_{1 / 2} \mathrm{CoO}_{2}$ (we still restrict the possibility of structural relaxations until later in this analysis). Fig. 2.8 shows the results with the frozen $U=0$ structures but now allowing for $\mathrm{CO}$ in $\mathrm{Li}_{1 / 2} \mathrm{CoO}_{2}$. Here again the formation energy increases with $U$. For small values of $U$ the increase is small and stems from the DFT contribution. After $\mathrm{CoO}_{2}$ orbitally orders and opens a band gap, the $U$-dc energetics are a phase separating contribution to the total formation energy. As before, essentially all of the $U$-dc contribution comes from the ordering, not the filling, of the $d$ orbitals. When $\mathrm{Li}_{1 / 2} \mathrm{CoO}_{2}$ charge orders for $U$ greater than $2 \mathrm{eV}$, the $U$-dc phase separating contribution is significantly dampened but there is always still a positive phase separating contribution (53-84 meV). CO also leads to an increase in the DFT formation energy contribution from $-0.34 \mathrm{eV}$ at $U=2 \mathrm{eV}$ to $-0.17 \mathrm{eV}$ at $U=3 \mathrm{eV}$.

As illustrated in Fig. 2.7(b) and 2.7(e), the variations in filling factor $\mu(1-\mu)$ are again negligible so the only appreciable component to the $U$-dc energetics stems from the changes in ordering factor $\sigma^{2}$. The $\mathrm{CO}$ of $\mathrm{Li}_{1 / 2} \mathrm{CoO}_{2}$ significantly increases the average $\sigma^{2}$, but it still always lags behind the average of those of $\mathrm{CoO}_{2}$ and $\mathrm{LiCoO}_{2}$. For example, for $U=5$ $\mathrm{eV} E_{\text {ord }}$ is $87 \mathrm{meV}$ for $x=1 / 2$ and $90 \mathrm{meV}$ for the average for the $x=0$ and $x=1$ values. 
In this case we still end up with a total formation energy that steadily increases with $U$, though now $\mathrm{CO}$ dampens the process substantially such that for $U=5 \mathrm{eV}$ the value remains negative consistent with experiment.
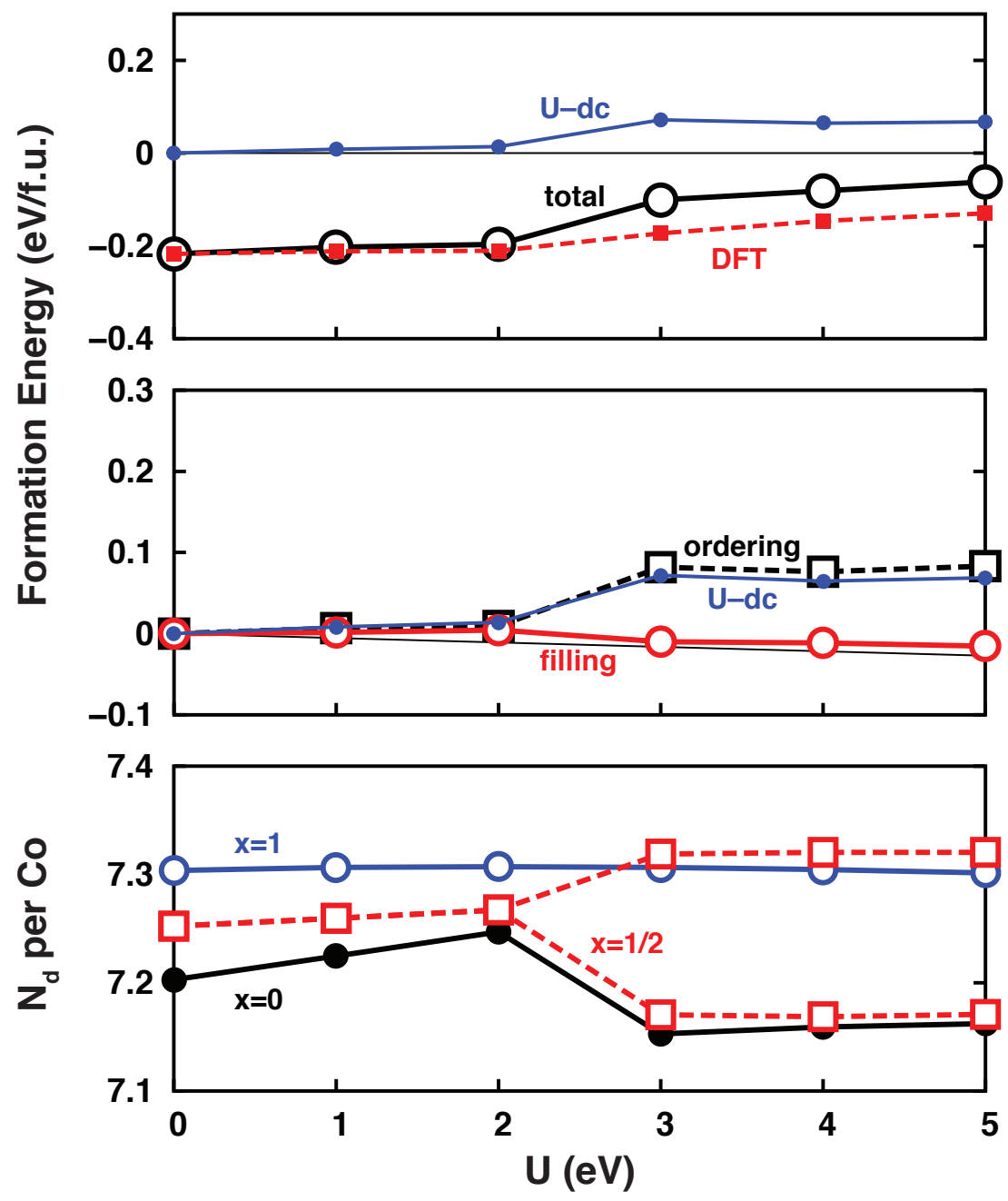

Figure 2.9: (a) Total $\mathrm{Li}_{1 / 2} \mathrm{CoO}_{2}$ formation energy (open black circles) and its DFT (filled red squares) and $U$-dc (filled blue circles) components as a function of $U$ (b) $\mathrm{Li}_{1 / 2} \mathrm{CoO}_{2} U$-dc (filled blue circles) formation energy component and its orbital filling (open red circles) and orbital ordering (open black squares) components as a function of $U$ (c) Number of $d$ electrons per Co as a function of $U$ for $\mathrm{CoO}_{2}$ (filled black circles), $\mathrm{Li}_{1 / 2} \mathrm{CoO}_{2}$ (open red squares), and $\mathrm{LiCoO}_{2}$ (open blue circles). All data correspond to the case of fully relaxed structures and the two lines in panel (c) for $\mathrm{Li}_{1 / 2} \mathrm{CoO}_{2}$ correspond to the two distinct Co sites. 
When we include $\mathrm{CO}$ in $\mathrm{Li}_{1 / 2} \mathrm{CoO}_{2}$ and full structural relaxations, we find the same fundamental effect as in the previous case only allowing CO: structural relaxations only provide a further dampening. As shown in Fig. 2.9, the total formation energy increases with $U$ and the positive contribution stems from the ordering component of the $U$-dc energetics. Here the $\mathrm{CO}$ and $\mathrm{CoO}_{2}$ orbital ordering both occur for $U>2 \mathrm{eV}$. The increase in the DFT formation energy contribution upon CO is dampened due to relaxations. The total formation energy remains negative at $-61 \mathrm{meV}$ at $U=5 \mathrm{eV}$. The plots of $\mu(1-\mu)$ and $\sigma^{2}$ are shown in Fig. 2.7(c) and 2.7(f), respectively. Structural relaxations serve to slightly enhance $\sigma^{2}$ for $\mathrm{CoO}_{2}$ and decrease the difference in $\sigma^{2}$ for the distinct Co sites in $\mathrm{Li}_{1 / 2} \mathrm{CoO}_{2}$.

These results have significant implications on the accuracy and robustness of the DFT $+U$ description of strongly correlated materials. Without $\mathrm{CO}$, which is not found in experiments on this class of materials, DFT $+U$ incorrectly predicts that $\mathrm{Li}_{x} \mathrm{CoO}_{2}$ phase separates once $U$ becomes appreciable. This is true even if we allow structural relaxations while suppressing $\mathrm{CO}$, in which case the total formation energy values are $-40 \mathrm{meV},+67 \mathrm{meV}$, and $+182 \mathrm{meV}$ for $U$ of 3,4 , and $5 \mathrm{eV}$, respectively. That $\mathrm{DFT}+U$ requires artificial $\mathrm{CO}$ to correctly capture the phase stable nature of $\mathrm{Li}_{x} \mathrm{CoO}_{2}$ is a significant weakness of this approach.

\subsubsection{Impact of $U$ on electronic structure of $\mathrm{Li}_{x} \mathrm{FePO}_{4}$}

The interaction $U$ has been computed as 3.7 and $4.9 \mathrm{eV}$ for $\mathrm{LiFePO}_{4}$ and $\mathrm{FePO}_{4}$, respectively [Zhou et al. (2004a)]. The variation of the band gap, total magnetization, and Fe magnetic moment as a function of $U$ for $\mathrm{Li}_{x} \mathrm{FePO}_{4}$ is illustrated in Fig. 2.10. The endmembers are both AFM so there is zero net magnetization. The Fe magnetic moment, which increases approximately linearly with $U$, is $4.0-4.4 \mu_{B}$ for $\mathrm{FePO}_{4}$ and 3.6-3.8 $\mu_{B}$ for $\mathrm{LiFePO}_{4}$ for $0 \mathrm{eV} \leq U \leq 6 \mathrm{eV}$. The endmembers are both insulating. For this $U$ range the band gaps increases from 1.0 to $2.7 \mathrm{eV}$ for $\mathrm{FePO}_{4}$ and 0.4 to $4.5 \mathrm{eV}$, a much larger range, for $\mathrm{LiFePO}_{4}$. Very similar results for the endmembers are found when one freezes the ions 


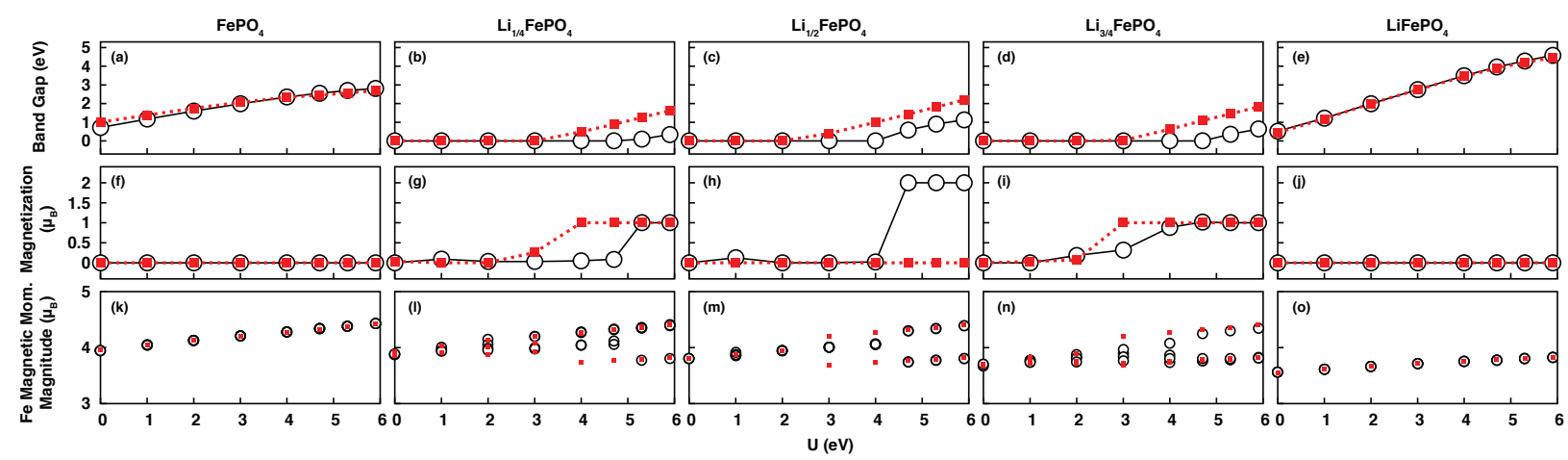

Figure 2.10: Electronic band gap as a function of $U$ for (a) $\mathrm{FePO}_{4}$, (b) $\mathrm{Li}_{1 / 4} \mathrm{FePO}_{4}$, (c) $\mathrm{Li}_{1 / 2} \mathrm{FePO}_{4}$, (d) $\mathrm{Li}_{3 / 4} \mathrm{FePO}_{4}$, and (e) $\mathrm{LiFePO}_{4}$ with relaxations (filled red squares) and with frozen linearly interpolated experimental structures (open black circles). The corresponding plots of total magnetization and Fe magnetic moment magnitudes are shown in panels $(\mathrm{f})-(\mathrm{j})$ and $(\mathrm{k})-(\mathrm{o})$, respectively. For panels $(\mathrm{k})-(\mathrm{o})$, the multiple symbols per $U$ correspond to the 4 distinct Fe sites.

to the experimental structures.

For intermediate $x$ we consider the lowest-energy configurations that fit within the primitive unit cell shown in Fig. 2.1(b). The structures correspond to removing Li2, Li3, and Li4 for $x=1 / 4, \operatorname{Li} 3$ and Li4 for $x=1 / 2$, and Li4 for $x=3 / 4$ [Zhou et al. (2004c)]. Calculations on the two other possible primitive cell $\mathrm{Li}_{1 / 2} \mathrm{FePO}_{4}$ structures are always found to be higher in energy, so we do not discuss them here. In addition to the case in which structures are fully relaxed, we also perform calculations on structures constructed via linear interpolation of the experimental $x=0$ and $x=1$ structures. This is an ideal manner to isolate the effect of lattice relaxations given that appreciable lattice distortions arise in the intermediate compounds even for $U=0$

For $U=0$ the intermediate $x$ species of $\mathrm{Li}_{x} \mathrm{FePO}_{4}$ are $\mathrm{AFM}$ metals without any CO. The Fe magnetic moments are $3.9,3.8$, and $3.7 \mu_{B}$ for $x=1 / 4, x=1 / 2$, and $x=3 / 4$, respectively. The magnetic moment magnitude gradually increases with $U$, and above critical $U$ values a $\mathrm{CO}$ transition occurs leading to distinct $\mathrm{Fe}^{3+}$-like $\left(d^{5}\right)$ and $\mathrm{Fe}^{2+}$-like $\left(d^{6}\right)$ sites within the primitive unit cell. This symmetry breaking leads to distinct local magnetic moments and the opening of an electronic band gap. Unlike $\mathrm{Li}_{x} \mathrm{CoO}_{2}$ for which $N_{d}$ differences among correlated 
sites are 0.15-0.18 electrons in the $\mathrm{CO}$ state, $\mathrm{Li}_{x} \mathrm{FePO}_{4}$ has $\mathrm{CO}$ states with substantially larger $N_{d}$ differences around 0.4 electrons due to the highly localized nature of this system.

For $\mathrm{Li}_{1 / 2} \mathrm{FePO}_{4}$ using the frozen structure (linear interpolation of endmember experimental structures), the $\mathrm{CO}$ transition at $U>4 \mathrm{eV}$ yields a ferrimagnetic state with total magnetization $2 \mu_{B}$; there are 2 Fe magnetic moments of $3.7 \mu_{B}\left(\mathrm{Fe}^{2+}\right.$-like $)$ and $2 \mathrm{Fe}$ magnetic moments of $-4.3 \mu_{B}\left(\mathrm{Fe}^{3+}\right.$-like). It opens a band gap of $0.6 \mathrm{eV}$ for $U=4.7 \mathrm{eV}$ that is further increased with $U$ to a value of $1.1 \mathrm{eV}$ for $U=5.9 \mathrm{eV}$. Including structural relaxations has little effect on the electronic structure in the regime of $U$ before the onset of CO. However, relaxations assist the $\mathrm{CO}$ transition and result in a lower critical value of $U=2 \mathrm{eV}$ above which the CO state remains AFM with Fe magnetic moments of \pm 3.7 and $\pm 4.2 \mu_{B}$. In addition, relaxation serves to enhance the band gap of the CO state to values of $0.4-2.2 \mathrm{eV}$.

$\mathrm{Li}_{1 / 4} \mathrm{FePO}_{4}$ and $\mathrm{Li}_{3 / 4} \mathrm{FePO}_{4}$ show similar behavior in which at critical values of $U$ (lower when structural relaxation is included) a CO transition opens an electronic band gap. For $\mathrm{Li}_{1 / 4} \mathrm{FePO}_{4}$ with (without) relaxations a band gap opens at $U=4 \mathrm{eV}(5.3 \mathrm{eV})$ when $\mathrm{CO}$ yields $1 \mathrm{Fe}^{3+}$-like site and $3 \mathrm{Fe}^{2+}$-like sites. For $\mathrm{Li}_{3 / 4} \mathrm{FePO}_{4}$ with (without) relaxations a band gap opens at $U=3 \mathrm{eV}(5.3 \mathrm{eV})$ when $\mathrm{CO}$ yields $3 \mathrm{Fe}^{3+}$-like sites and $1 \mathrm{Fe}^{2+}$-like site. For $x=1 / 4$ and $x=3 / 4$ the magnitude of the band gap is around $0.1-0.6 \mathrm{eV}$ for the frozen structures and a larger values of $0.5-1.8 \mathrm{eV}$ including relaxations. For these $x$, the CO transition always leads to a ferrimagnetic state with magnetization of $1 \mu_{B}$. The Fe magnetic moments of the distinct sites are around 3.8 and $4.4 \mu_{B}$ and slowly increase with $U$ as in the case of $x=1 / 2$. Differences in the magnetic moments between the frozen and relaxed structures in the CO regime are small. We note that for these $x$, unlike in $x=1 / 2$, we find partial $\mathrm{CO}$ for intermediate values of $U$ in which the Fe magnetic moments begin to take on slightly different values without the presence of a band gap. For example, including relaxations for $U=1 \mathrm{eV} \mathrm{Li}{ }_{1 / 4} \mathrm{FePO}_{4}$ is metallic with Fe magnetic moments of 3.9, -3.9 , -4.0, and $4.0 \mu_{B}$. This indicates $\mathrm{DFT}+U$ is driving $\mathrm{Li}_{x} \mathrm{FePO}_{4}$ towards $\mathrm{CO}$ even for small $U$, which is to be expected given the very narrow bandwidths. 

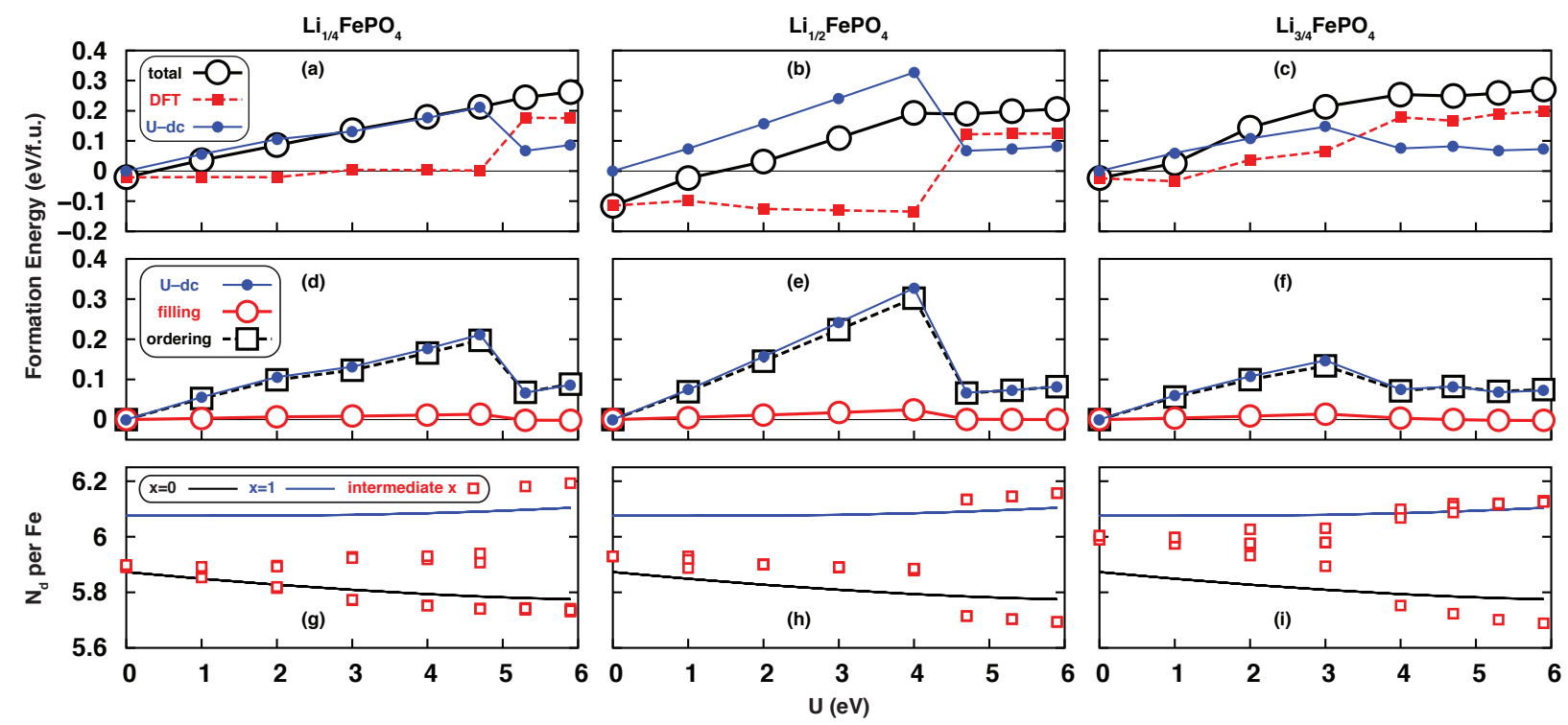

Figure 2.11: Total $\mathrm{Li}_{x} \mathrm{FePO}_{4}$ formation energy (open black circles) and its DFT (filled red squares) and $U$-dc (filled blue circles) components as a function of $U$ for (a) $x=1 / 4$, (b), $1 / 2$, and (c) $x=3 / 4$. (d)-(f) show the corresponding plots of $\mathrm{Li}_{x} \mathrm{FePO}_{4} U$-dc (filled blue circles) formation energy component and its orbital filling (open red circles) and orbital ordering (open black squares) components as a function of $U$. (g)-(i) show the corresponding plots of number of $d$ electrons per $\mathrm{Fe}$ as a function of $U$ for $\mathrm{FePO}_{4}$ (black line), $\mathrm{Li}_{x} \mathrm{FePO}_{4}$ (open red squares), and $\mathrm{LiFePO}_{4}$ (blue line). All data correspond to the case of frozen linearly interpolated experimental structures.

Unlike for $\mathrm{Li}_{x} \mathrm{CoO}_{2}$ (see Fig. 2.4), for $\mathrm{Li}_{x} \mathrm{FePO}_{4}$ the $\mathrm{CO}$ is stabilized by structural relaxations as evidenced by the lower critical $U$ values for CO. The stabilization of CO by structural relaxations suggests stronger coupling between the electronic and lattice degrees of freedom in $\mathrm{Li}_{x} \mathrm{FePO}_{4}$ as compared to $\mathrm{Li}_{x} \mathrm{CoO}_{2}$. This is consistent with the evidence for polarons in $\mathrm{Li}_{x} \mathrm{FePO}_{4}$ [Ellis et al. (2006); Zaghib et al. (2007)].

\subsubsection{Impact of $U$ on phase stability of $\mathrm{Li}_{x} \mathrm{FePO}_{4}$}

The formation energy behavior for $\mathrm{Li}_{x} \mathrm{FePO}_{4}$ as a function of $U$ without allowing for the effect of structural relaxations is summarized in Fig. 2.11. The behavior shares many 
similarities and a few differences to that of $\mathrm{Li}_{x} \mathrm{CoO}_{2}$, which we will describe. As illustrated in panels (a)-(c), for all intermediate $x$ the total formation energy increases with $U$ as in the case of $\mathrm{Li}_{x} \mathrm{CoO}_{2}$ and changes sign from negative to positive for sufficiently high $U$. For example, for $x=1 / 2$ the formation energy is $-0.11 \mathrm{eV}$ at $U=0$ and increases to $+0.21 \mathrm{eV}$ for $U=5.9 \mathrm{eV}$. For $x=1 / 4$ and $x=3 / 4$, there are only slightly negative values of around $-0.02 \mathrm{eV}$ for $U=0$, which increase to as much as $+0.26-0.27 \mathrm{eV}$ as $U$ increases. We note that the formation energies are higher for this frozen structure case since the intermediate $x$ species will exhibit more significant structural relaxations than the endmembers.

For most of the $U$ range, that below the critical $U$ for $\mathrm{CO}$, the DFT component of the formation energy is approximately constant and does not strongly influence phase stability. For example, for $x=1 / 4$ this component varies by only $25 \mathrm{meV}$ for $0 \mathrm{eV} \leq U \leq 4.7 \mathrm{eV}$. In contrast, the $U$-dc component of the formation energy undergoes significant changes as it increases roughly linearly with $U$; the change is $0.21 \mathrm{eV}$ over the same $U$ range for $x=1 / 4$. This $U$-dc contribution is positive and therefore, as in the case of $\mathrm{Li}_{x} \mathrm{CoO}_{2}$, is what drives the total formation energy towards phase separation in $\mathrm{Li}_{x} \mathrm{FePO}_{4}$ in this regime before CO. One caveat to this characterization is that the DFT component varies more considerably (by $90 \mathrm{meV}$ ) for $\mathrm{Li}_{3 / 4} \mathrm{FePO}_{4}$ in this regime. In this case it is positive for $2 \mathrm{eV} \leq U \leq 3 \mathrm{eV}$ and thus can be described as partially responsible for the positive total formation energy.

Upon CO there is a drastic change in the formation energy components, as in the case of $\mathrm{Li}_{x} \mathrm{CoO}_{2}$. The $U-\mathrm{dc}$ component drops steeply to much smaller values of around $0.06-0.08 \mathrm{eV}$. For $x=1 / 2$, for example, the value is $0.33 \mathrm{eV}$ for $U=4 \mathrm{eV}$ and only $0.07 \mathrm{eV}$ for $U=4.7 \mathrm{eV}$ due to the $\mathrm{CO}$ transition; the drop in the $U$-dc contribution is substantial though smaller in magnitude for $x=1 / 4$ and $x=3 / 4$. Here as in $\mathrm{Li}_{x} \mathrm{CoO}_{2}$ we also find an increase in the DFT formation energy contribution due to CO. For example, for $\mathrm{Li}_{1 / 2} \mathrm{FePO}_{4}$ the DFT formation energy contribution jumps from $-135 \mathrm{meV}$ to $+122 \mathrm{meV}$ across the $\mathrm{CO}$ phase boundary. Unlike in $\mathrm{Li}_{1 / 2} \mathrm{CoO}_{2}$ [see Fig. 2.8(a)], for $\mathrm{Li}_{1 / 2} \mathrm{FePO}_{4}$ the increase in the DFT component is enough to make it positive. It is even larger in magnitude than the $U$-dc component. For 
$x=1 / 4$ and $x=3 / 4$ the DFT component is already positive but it becomes substantially more positive after CO. Despite how sharp the changes in formation energy components are, the total formation energy changes less abruptly from CO. For example, for $x=1 / 2$ the total formation energy changes only by $3 \mathrm{meV}$ between $U=4 \mathrm{eV}$ and $U=4.7 \mathrm{eV}$. After the CO transition, the formation energy and its components are relatively flat versus $U$. For $x=1 / 2$, for example, the changes are on the order of only $15-25 \mathrm{meV}$.

The breakdown of $U$-dc formation energy into filling and ordering contributions is shown in Fig. 2.11(d)-(f). As in $\mathrm{Li}_{x} \mathrm{CoO}_{2}$, it can be seen that the average filling contributes negligibly to the phase separation; the maximum contribution is at most $25 \mathrm{meV}$ and typically much smaller. Therefore, again the impact of $U$ and the dc is essentially entirely contained within the ordering contribution. The ordering contribution tracks the behavior of the total $U$-dc term and it can be as much as $+0.3 \mathrm{eV}$.

The plots of $N_{d}$ on each Fe site versus $U$ in Fig. 2.11(g)-(i) illustrate that there is also a $p-d$ rehybridization mechanism in $\mathrm{Li}_{x} \mathrm{FePO}_{4}$ similar but smaller than that in $\mathrm{Li}_{x} \mathrm{CoO}_{2}$ with differences in $N_{d}$ around 20-30\% of a full electron between $x=0$ and $x=1$. Like the Fe magnetic moment data in Fig. 2.10, these $N_{d}$ values illustrate the partial and full CO that occurs as $U$ increases. We note that without structural relaxations the spread in $N_{d}$ values after $\mathrm{CO}$ for the intermediate $x$ can be larger than the difference between $N_{d}$ of $x=0$ and $x=1$.

To investigate the origin of the positive $U$-dc phase-separating contribution to the total formation energy for $\mathrm{Li}_{x} \mathrm{FePO}_{4}$, in Fig. 2.12 we plot the individual $\mu(1-\mu)$ [panel (a)] and $\sigma^{2}$ [panel (c)] values as a function of $x$ for different $U$. For clarity, we take the average over the $4 \mathrm{Fe}$ sites in the primitive unit cell. As in the case of $\mathrm{Li}_{x} \mathrm{CoO}_{2}$, the magnitude of the filling factor is high (around 0.24 ) but the changes with respect to the average of the endmember values are very small (on the order of $10^{-3}$ ). This is responsible for the negligible contribution of the filling component to the total formation energy. As for $\mathrm{Li}_{x} \mathrm{CoO}_{2}$, the filling factor is highest for $x=0$ and $x=1$. Compared to those of $\mathrm{Li}_{x} \mathrm{CoO}_{2}$, the $\mu(1-\mu)$ values of $\mathrm{Li}_{x} \mathrm{FePO}_{4}$ 


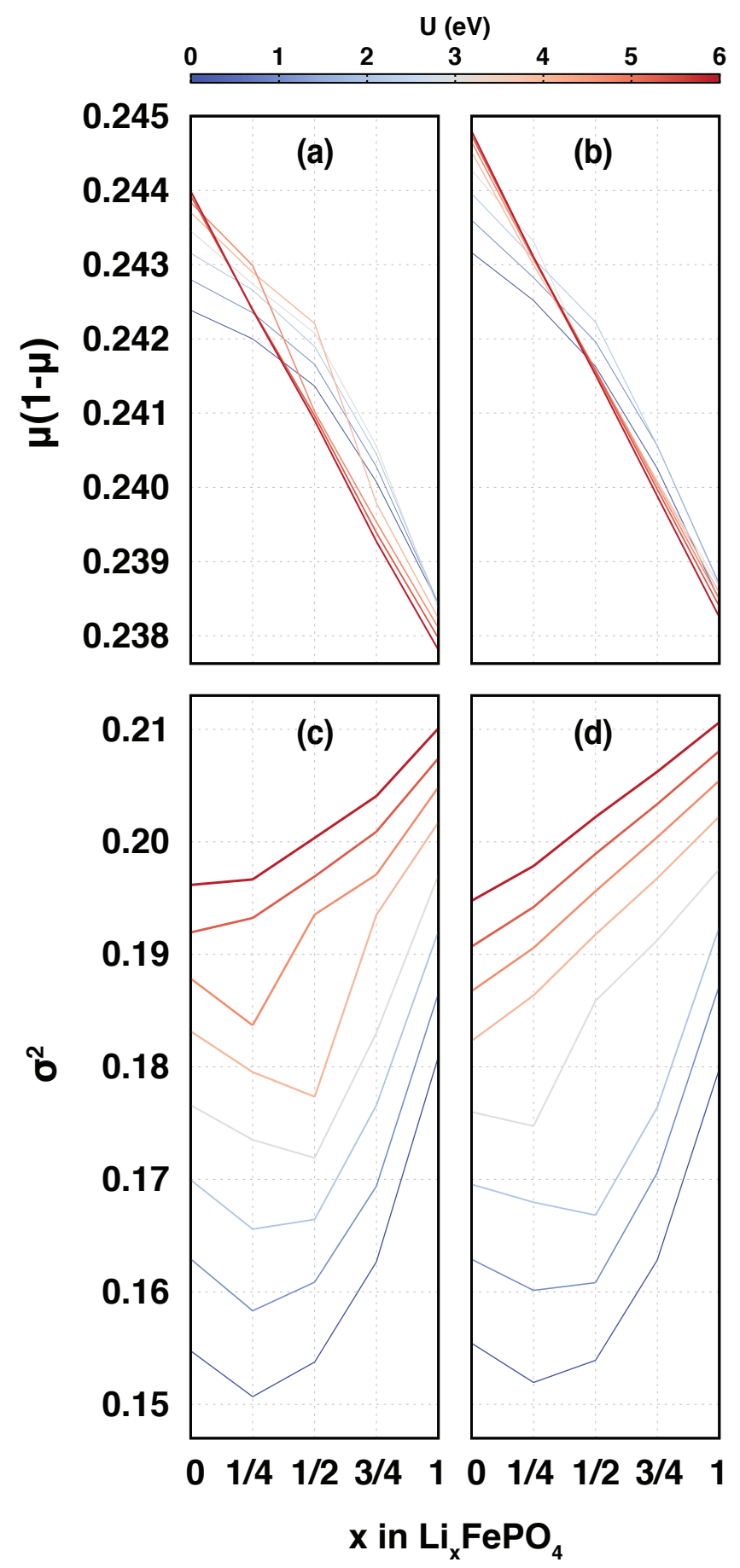

Figure 2.12: Filling factor $\mu(1-\mu)$ as a function of $x$ averaged over Fe sites for different $U$ in $\mathrm{Li}_{x} \mathrm{FePO}_{4}$ for (a) linearly interpolated experimental structures and (b) relaxed structures. Panels (c) and (d) show the corresponding plots for the ordering factor $\sigma^{2}$. The line thickness increases for increasing values of $U$. 
are much nearer to the range expected from nominal electron counting for $\mathrm{Fe}^{2+}$ and $\mathrm{Fe}^{3+}$ (0.24-0.25) since there is less hybridization between $p$ and $d$ states. This is also the reason for the enhanced magnitude of $\sigma^{2}$ compared to that of $\mathrm{Li}_{x} \mathrm{CoO}_{2}$.

Compared to $\mu(1-\mu), \sigma^{2}$ has a smaller but still significant magnitude (around 0.18) for $\mathrm{Li}_{x} \mathrm{FePO}_{4}$. The $\sigma^{2}$ values monotonically increase with $U$ (by around $0.003-0.006$ per eV) for all $x$, which as in the case of $\mathrm{Li}_{x} \mathrm{CoO}_{2}$ is expected since the $U$ and dc terms serve to enhance orbital polarization in the correlated subspace. Unlike in $\mu(1-\mu)$, there are substantial deviations of the $\sigma^{2}$ values for intermediate $x$ compared to the endmember average. For example, for $x=1 / 2$ at $U=3 \mathrm{eV} \sigma^{2}=0.172$ as compared to 0.187 for the endmember average. The ordering factor is consistently lower than the endmember linear interpolation, thus leading to the positive formation energy contribution.

One can observe a moderate increase in $\sigma^{2}$ values for intermediate $x$ upon CO. For example, for $x=1 / 2 \sigma^{2}$ jumps from 0.177 to 0.194 upon $\mathrm{CO}$ at $U=4.7 \mathrm{eV}$. However, as in the case of $\mathrm{Li}_{x} \mathrm{CoO}_{2}$ the $\sigma^{2}$ values still are always lower than the linear interpolation of $x=0$ and $x=1$ values even after CO. For example, the endmember average $\sigma^{2}$ is 0.196 for $U=4.7 \mathrm{eV}$ so it is still slightly larger than the value for $\mathrm{Li}_{1 / 2} \mathrm{FePO}_{4}$. Therefore, we find that $\mathrm{CO}$ alleviates but does not eliminate the tendency for phase separation with $U$ derived from the ordering of correlated orbitals.

Figure 2.13(a)-(c) shows the formation energy behavior of $\mathrm{Li}_{x} \mathrm{FePO}_{4}$ including full structural relaxations. In this case, since there are more significant total energy lowerings from relaxing the intermediate $x$ structures compared to those from relaxing the endmembers, the total formation energy values are significantly lower. For example, for $\mathrm{Li}_{1 / 4} \mathrm{FePO}_{4}$ the maximum formation energy is $52 \mathrm{meV}$ as opposed to $262 \mathrm{meV}$ in the case of linearly interpolated endmember experimental structures. Otherwise, the behavior is generally similar to the case without relaxations. For all $x$, the total formation energy increases monotonically with $U$ and for sufficiently high $U$ switches from negative to positive. We note that the formation energy $\mathrm{Li}_{1 / 2} \mathrm{FePO}_{4}$ is only slightly positive $(+6.7 \mathrm{meV})$ for the largest $U$ we considered. This 

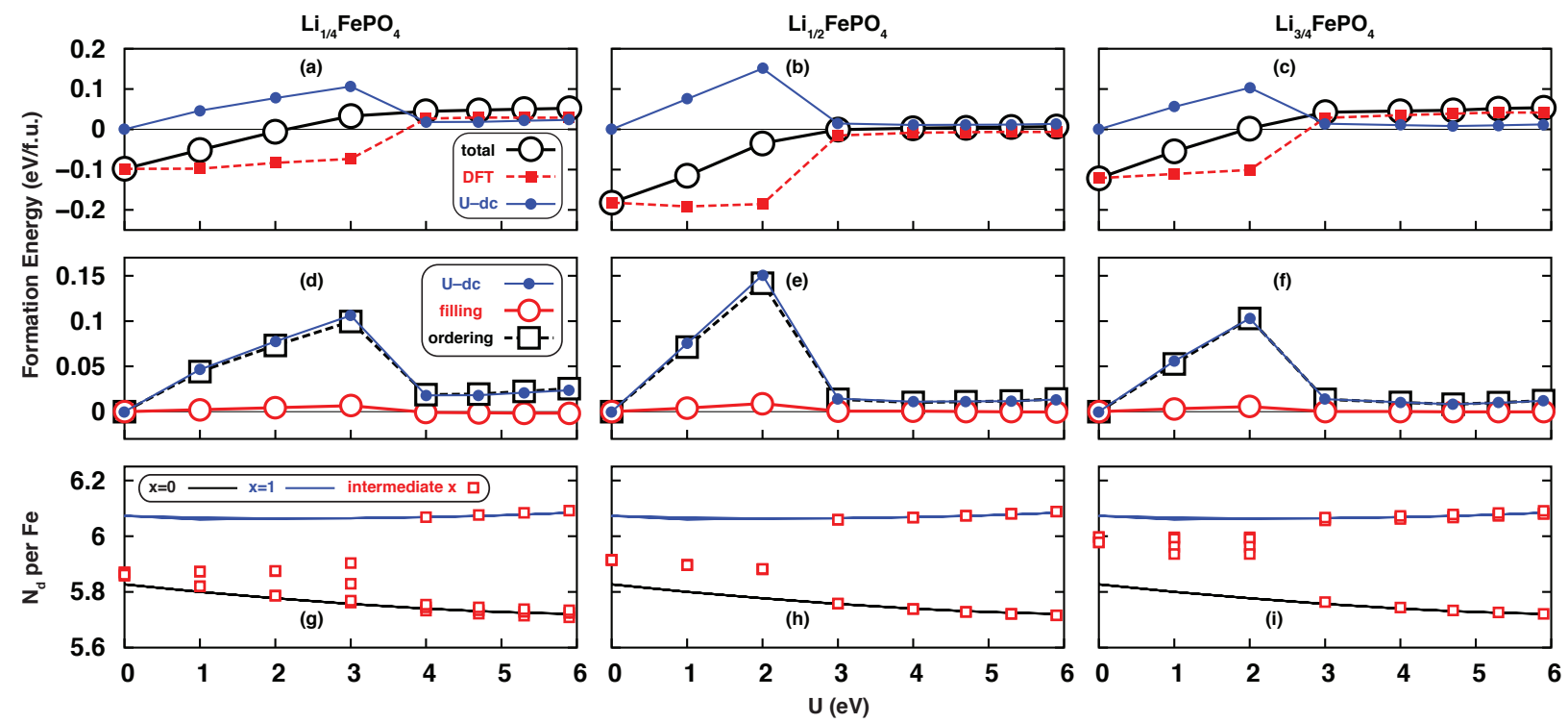

Figure 2.13: Total $\mathrm{Li}_{x} \mathrm{FePO}_{4}$ formation energy (open black circles) and its DFT (filled red squares) and $U$-dc (filled blue circles) components as a function of $U$ for (a) $x=1 / 4$, (b), $1 / 2$, and (c) $x=3 / 4$. (d)-(f) show the corresponding plots of $\mathrm{Li}_{x} \mathrm{FePO}_{4} U$-dc (filled blue circles) formation energy component and its orbital filling (open red circles) and orbital ordering (open black squares) components as a function of $U$. (g)-(i) show the corresponding plots of number of $d$ electrons per $\mathrm{Fe}$ as a function of $U$ for $\mathrm{FePO}_{4}$ (black line), $\mathrm{Li}_{x} \mathrm{FePO}_{4}$ (open red squares), and $\mathrm{LiFePO}_{4}$ (blue line). All data correspond to the case of relaxed structures.

is in quantitative disagreement with the original DFT $+U$ work [Zhou et al. (2004c)] for reasons which are not clear, but is consistent with a more recent report [Ong et al. (2011)].

Here again the DFT formation energy contribution is approximately constant (deviations of at most $25 \mathrm{meV}$ and typically less) before the $\mathrm{CO}$ transition with a slight tendency to increase with $U$. In this case $x=3 / 4$ is no longer an exception to the general trend. In contrast, the $U$-dc contribution is positive and significantly increases (roughly linearly) with $U$. The largest value it takes on is $151 \mathrm{meV}$ for $x=1 / 2$ at $U=2 \mathrm{eV}$. Therefore, again it is the positive $U$-dc contribution that destabilizes the compounds of intermediate $x$ in this regime.

After $\mathrm{CO}$, the $U$-dc formation energy contribution is significantly dampened but remains 
positive. For example, for $x=1 / 4$ it decreases from 106 to $18 \mathrm{meV}$ at the $\mathrm{CO}$ phase boundary. The effect is similarly substantial for all $x$. At the same time, the DFT formation energy component abruptly increases due to $\mathrm{CO}$ and becomes positive $(x=1 / 4$ and $x=$ $3 / 4)$ or much less negative $(x=1 / 2)$. The magnitude of this increase is more substantial than that of $\mathrm{Li}_{x} \mathrm{CoO}_{2}$, which suggests that in $\mathrm{Li}_{x} \mathrm{FePO}_{4} \mathrm{CO}$ constitutes a more significant rearrangement of charge density. The net effect is that upon $\mathrm{CO}$ the increase in total formation energy with $U$ is slowed. For the $x=1 / 4$ case, for example, the total formation energy increases only by $12 \mathrm{meV}$ from $U=3$ to $U=4 \mathrm{eV}$ as opposed to the lower- $U$ regime in which the same change in $U$ yields increases of around $46 \mathrm{meV}$. In the CO state both the DFT and $U$-de components of the formation energy are nearly constant with respect to $U$. As such, the total formation energies saturate to around $+50,+6$, and $+50 \mathrm{meV}$ for $x=1 / 4, x=1 / 2$, and $x=3 / 4$ in the regime of large $U$.

As illustrated in Fig. 2.13(d)-(f), in this case again the positive $U$-dc formation energy contribution stems entirely from the ordering contribution (from the spread in orbital occupancies); the filling component (from the average orbital occupancy) is negligible with values less than $10 \mathrm{meV}$. CO lowers the filling contribution even further to no more than $1 \mathrm{meV}$. In contrast, the ordering contribution is dampened but still positive and appreciable; it tracks the behavior of the total $U$-dc formation energy contribution.

The evolution of the $N_{d}$ values into two discrete groups $\left(\mathrm{Fe}^{3+}-\right.$ and $\mathrm{Fe}^{2+}$-like $)$ due to $\mathrm{CO}$ is shown in Fig. 2.13(g)-(i). A similar magnitude of $p$ - $d$ rehybridization is observed based on the overall range of $N_{d}$. As in the case without structural relaxations, here for $x=1 / 4$ and $x=3 / 4$ though not $x=1 / 2$ there is a regime of intermediate $x$ in which there is partial CO in metallic states. After the CO transition the two groups of $N_{d}$ values closely match those of $x=0$ and $x=1$, which suggests the CO is very complete and the local environments of Fe for intermediate $x$ mimic those of the endmembers.

The filling and ordering factors for relaxed $\mathrm{Li}_{x} \mathrm{FePO}_{4}$ are shown in Fig. 2.12(b) and 2.12(d), respectively. We find similar results when including structural relaxations. The 
deviations in $\mu(1-\mu)$ with respect to the linear interpolation of endmember values are negligible. $\sigma^{2}$ gradually increases with $U$ and the values for intermediate $x$ always lag behind the endmember linear interpolation; the effect is substantially dampened but not entirely eliminated when CO increases $\sigma^{2}$ for intermediate $x$. We note that with structural relaxations after CO the $\sigma^{2}$ versus $x$ curves are nearly linear, which illustrates that relaxations provide a further dampening of the general tendency towards phase separation with $U$ stemming from the ordering term.

\subsubsection{Phase stability of $\mathrm{Li}_{x} \mathrm{CoPO}_{4}$}

To further validate our general understanding of the impact of $U$ on phase stability, we investigate $\mathrm{Li}_{x} \mathrm{CoPO}_{4}$. This material is isostructural to the olivine $\mathrm{Li}_{x} \mathrm{FePO}_{4}$ structure shown in Fig. 2.1 with (nominally) an additional electron on the transition metal site. $\mathrm{Li}_{x} \mathrm{CoPO}_{4}$ is of interest since it has been shown to have a very high voltage $(4.8 \mathrm{~V})$ as a cathode material [Amine et al. (2000)]. It is intriguing physically since unlike $\mathrm{Li}_{x} \mathrm{FePO}_{4}$ it does have a stable intermediate compound, for $x \approx 2 / 3$ [Bramnik et al. (2007); Ehrenberg et al. (2009); Strobridge et al. (2014)].

We consider the lowest-energy configuration of $\mathrm{Li}_{2 / 3} \mathrm{CoPO}_{4}$ deduced by Strobridge et al. [Strobridge et al. (2014)] and study the formation energy as a function of $U$ with the frozen $U=0$ structures. Experimentally the $\mathrm{Li}_{x} \mathrm{CoPO}_{4}$ system is AFM [Santoro et al. (1966); Ehrenberg et al. (2009)], so our calculations consider the endmembers in the AFM state. We base our calculations on the magnetic configuration of $\mathrm{LiCoPO}_{4}$, which is identical to that of $\mathrm{LiFePO}_{4}$ [Santoro et al. (1966); Santoro and Newnham (1967)]. For $\mathrm{Li}_{2 / 3} \mathrm{CoPO}_{4}$ we find the AFM state to be unstable and devolves into a ferrimagnetic state. In this state one of the Co sites has no magnetic moment unlike the $\pm \approx 2.6-2.7 \mu_{B}$ values of the other 11 leading to a total magnetization of $\approx 3.2 \mu_{B}$.

We find total formation energies of $-0.18,-0.14$, and $-0.03 \mathrm{eV}$ for $U$ values of 0,2 , and 
$5.48 \mathrm{eV}$, respectively. This corresponds to the same trend of formation energy increasing with $U$. We again find the positive formation energy contribution is the $U$-dc component, and here also the filling contribution is very small (magnitude of at most $20 \mathrm{meV}$ ) so the origin is increased ordering of the endmember $d$ states relative to those of the intermediate $x$ species.

For the above analysis we have restricted our attention to the AFM or AFM-like states as they are the experimental magnetic structure for $\mathrm{Li}_{x} \mathrm{CoPO}_{4}$. However, we note that the magnetic ground state of $\mathrm{Li}_{x} \mathrm{CoPO}_{4}$ predicted by $\mathrm{DFT}+U$ changes as a function of $U$, which is likely the origin of a previous study finding the formation energy becomes more negative as a function of $U$ [Strobridge et al. (2014)]. For $\mathrm{LiCoPO}_{4}$ we find a ferromagnetic (FM) ground state within DFT with the AFM state 37 meV higher in energy, whereas for $\mathrm{CoPO}_{4}$ we find a non-spin-polarized (NSP) ground state with the FM and AFM states 57-61 meV higher in energy. At $U=2$ the ground state of $\mathrm{LiCoPO}_{4}$ becomes antiferromagnetic, and by $U=5.5 \mathrm{eV}$ the ground state for both endmembers is AFM consistent with experiments. For $x=2 / 3$ we find the FM state is unstable within DFT and the NSP state is $0.54 \mathrm{eV}$ higher in energy than the ferrimagnetic state. At $U=2 \mathrm{eV}$ the FM state becomes metastable only $7 \mathrm{meV}$ above the ferrimagnetic state, and for $U=5.5 \mathrm{eV}$ the FM state becomes the ground state with the ferrimagnetic state $68 \mathrm{meV}$ high in energy.

\subsubsection{Impact of double counting on phase separation trend}

Our preceding analysis shows that it is valuable to recast the $U$-dc energy as the sum of ordering and filling terms, as the filling term was shown to have negligible impact on formation energies. However, there is still utility in analyzing what can be learned by separately inspecting $E_{U}$ and $E_{d c}$, given that the $E_{U}$ term specifically is handled much more precisely in the context of DFT+DMFT.

The simplest possible explanation for the positive $U$-dc formation energy contribution 
comes from the form of the dc term Eq. 2.4. We expect the $\mathrm{Li}$ in $\mathrm{Li}_{x} \mathrm{CoO}_{2}$ and other intercalation materials will be ionized and donate some amount of charge (depending on the degree of hybridization with $\mathrm{O} p$ states) to Co. Therefore, we might make the assumption that $N_{d}$ is linear in $x$. Since energy contributions linear in $x$ do not contribute to the formation energy by the definition of Eq. 2.11, this means the term linear in $N_{d}$ in the dc term cannot contribute to the formation energy. The other part of the dc, however, yields a term proportional to $-N_{d}^{2}$ (a negative quadratic in $N_{d}$ ) in the total energy. With the assumption of a linear relationship between $N_{d}$ and $x$, this term is a negative quadratic in $x$ that necessarily provides a positive formation energy contribution.

This simple kind of argument fails to fully describe the observed behavior for the following reasons, which we illustrate using the simplest example of $\mathrm{Li}_{1 / 2} \mathrm{CoO}_{2}$ without $\mathrm{CO}$ considering in the frozen $U=0$ structures. The first two reasons come from the fact that the variation of $N_{d}$ with $x$ is in fact substantially nonlinear, as can be easily seen for the $U>1 \mathrm{eV}$ data in Fig. 2.6(c). In this regime, $N_{d}$ for $x=1 / 2$ is much closer to the $x=1$ value than the $x=0$ value. For example, for $U=5 \mathrm{eV}$ the $N_{d}$ values are 7.19, 7.28, and 7.30 for $x=0, x=1 / 2$, and $x=1$, respectively. First of all, this means that the contribution of the dc energy that is proportional to $N_{d}$ will contribute to the formation energy. For example, for this case it takes on the value of $+89 \mathrm{meV}$ for $U=5 \mathrm{eV}$.

Secondly, the quadratic part of the dc energy no longer necessarily gives a positive formation energy contribution for $N_{d}$ that is nonlinear in $x$. One can parametrize the deviation from linearity using the additive form $N_{d}(x)=\overline{N_{d}(x)}+\delta_{x}$, where $N_{d}(x)$ is the $N_{d}$ value for Li concentration $x, \overline{N_{d}(x)}=(1-x) N_{d}(0)+x N_{d}(1)$ is the linear value of $N_{d}(x)$, and $\delta_{x}$ is the deviation from linearity. Here we assume a single site for clarity. It then can be shown that 
the formation energy contribution stemming from the quadratic part of the dc energy is

$$
\begin{aligned}
F E_{d c}^{q u a d}(x)=\frac{1}{2} U\{ & x(1-x)\left[N_{d}(1)-N_{d}(0)\right]^{2} \\
& \left.-2 \overline{N_{d}(x)} \delta_{x}\left(1+\frac{\delta_{x}}{2 \overline{N_{d}(x)}}\right)\right\} .
\end{aligned}
$$

Note that the term proportional to $\delta_{x}^{2}$ can be ignored as $\delta_{x}$ should be much smaller than $2 \overline{N_{d}(x)}$. When $\delta_{x}=0\left(N_{d}\right.$ is linear with $\left.x\right)$, we indeed have a positive formation energy contribution since the second term vanishes and the first term will be positive since $N_{d}(0) \neq$ $N_{d}(1)$. However, this second term can lead to a significant negative (phase stabilizing) formation energy contribution when $\delta_{x}$ is positive and non-negligible. This actually results in a strongly negative formation energy contribution in the present case: for $U=5 \mathrm{eV}$, for example, the formation energy contribution from the quadratic part of the dc is $-1.29 \mathrm{eV}$.

The third and final reason the simple argument fails is that the $E_{U}$ term also contributes significantly to the formation energy. For example, for $U=5 \mathrm{eV}$ the formation energy contribution from the interaction term is $+1.78 \mathrm{eV}$. Therefore, the dc term is not responsible for the observed trend towards phase separation.

\subsubsection{Average intercalation voltage of $\mathrm{Li}_{x} \mathrm{CoO}_{2}$ and $\mathrm{Li}_{x} \mathrm{FePO}_{4}$}

Up to now our analysis of the DFT $+U$ energetics has focused on the formation energy, which cannot straightforwardly be assessed experimentally, limiting the degree to which we can quantitatively scrutinize $\mathrm{DFT}+U$ energetics. Therefore, we now turn to average intercalation voltage. This quantity, which is a function of the energy difference of the endmembers and the energy of bulk Li, can be measured experimentally.

Figure 2.14 shows the behavior of the average intercalation voltage as a function of $U$. As found previously, the voltages tend to increase with $U$ for both $\mathrm{Li}_{x} \mathrm{CoO}_{2}$ and $\mathrm{Li}_{x} \mathrm{FePO}_{4}$ [Zhou et al. (2004a)]. For $\mathrm{Li}_{x} \mathrm{CoO}_{2}$ using relaxed structures, the computed voltage exhibits 


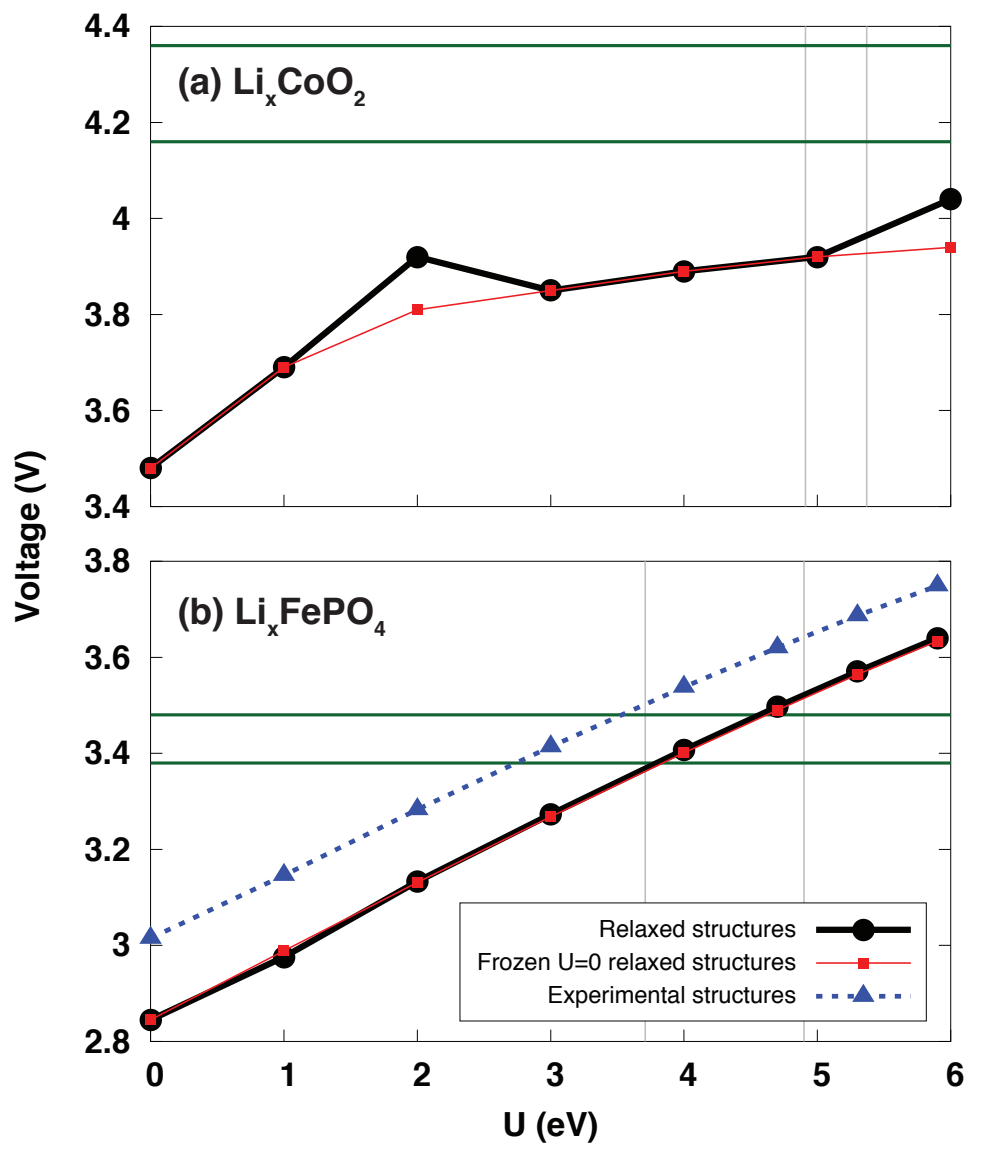

Figure 2.14: Average intercalation voltage of (a) $\mathrm{Li}_{x} \mathrm{CoO}_{2}$ and (b) $\mathrm{Li}_{x} \mathrm{FePO}_{4}$ as a function of $U$ shown using relaxed structures and frozen $U=0$ relaxed structures. For $\mathrm{Li}_{x} \mathrm{FePO}_{4}$ the results using the experimental structures are also shown. Green lines indicate the range of the average voltage measured from experiment, and grey lines indicate $U$ values of the endmembers computed via the linear response method [Zhou et al. (2004a)]. 
one discontinuity at $U=2 \mathrm{eV}$ after which $\mathrm{CoO}_{2}$ gaps and another at $U=5 \mathrm{eV}$ after which $\mathrm{LiCoO}_{2}$ becomes high spin. Otherwise, the values are almost identical to those found using the frozen $U=0$ structures. The DFT value is $3.48 \mathrm{~V}$ and there is an increase to 3.92 $\mathrm{V}$ at $U=5 \mathrm{eV}$. We note that the predicted voltages for $\mathrm{Li}_{x} \mathrm{CoO}_{2}$ are smaller than those reported in the work of Zhou et al. [Zhou et al. (2004a)], but agree with several more recent studies [Chevrier et al. (2010); Aykol and Wolverton (2014); Aykol et al. (2015)]. Ultimately, $\mathrm{DFT}+U$ underpredicts the average voltage of $\mathrm{Li}_{x} \mathrm{CoO}_{2}$ compared to the experimental value of $4.26 \mathrm{~V}$ [Amatucci et al. (1996)]. For $\mathrm{Li}_{x} \mathrm{FePO}_{4}$ using relaxed structures, the DFT voltage is $2.85 \mathrm{~V}$ and increases approximately linearly to $3.50 \mathrm{~V}$ at $U=4.7 \mathrm{eV}$, in agreement with the experimental voltage of $3.43 \mathrm{~V}$ [Padhi et al. (1997a); Yamada et al. (2001)]. Using the experimental structures, the predicted intercalation voltage is enhanced by $0.1-0.2 \mathrm{~V}$.

\subsubsection{Li order-disorder transition temperature of $\mathrm{Li}_{1 / 2} \mathrm{CoO}_{2}$}

Another observable with which we can assess accuracy of DFT $+U$ energetics, albeit at a fixed composition, is the order-disorder (O-D) transition temperature for Li. This is the temperature above which the Li ions and vacancies become disordered. Here we consider $\mathrm{Li}_{1 / 2} \mathrm{CoO}_{2}$, whose experimental O-D temperature is $333 \mathrm{~K}$ [Reimers and Dahn (1992)].

We estimate the O-D transition temperature based on the ground state total energies of the ordered and disordered (SQS) phases using the equation

$$
T_{O-D}=\frac{E_{D}-E_{O}}{k_{B} \ln (2)}
$$

where $k_{B}$ is the Boltzmann constant and the factor of $\ln (2)$ comes from the entropy of mixing for $x=1 / 2$. This expression is an approximation to performing finite-temperature Monte Carlo simulations on a cluster expansion based on such ground-state total energies. Past

work suggests that this simpler expression should reasonably capture trends in $T_{O-D}[\mathrm{Lu}$ et al. (1994); Jiang et al. (2004, 2005, 2009)]. 


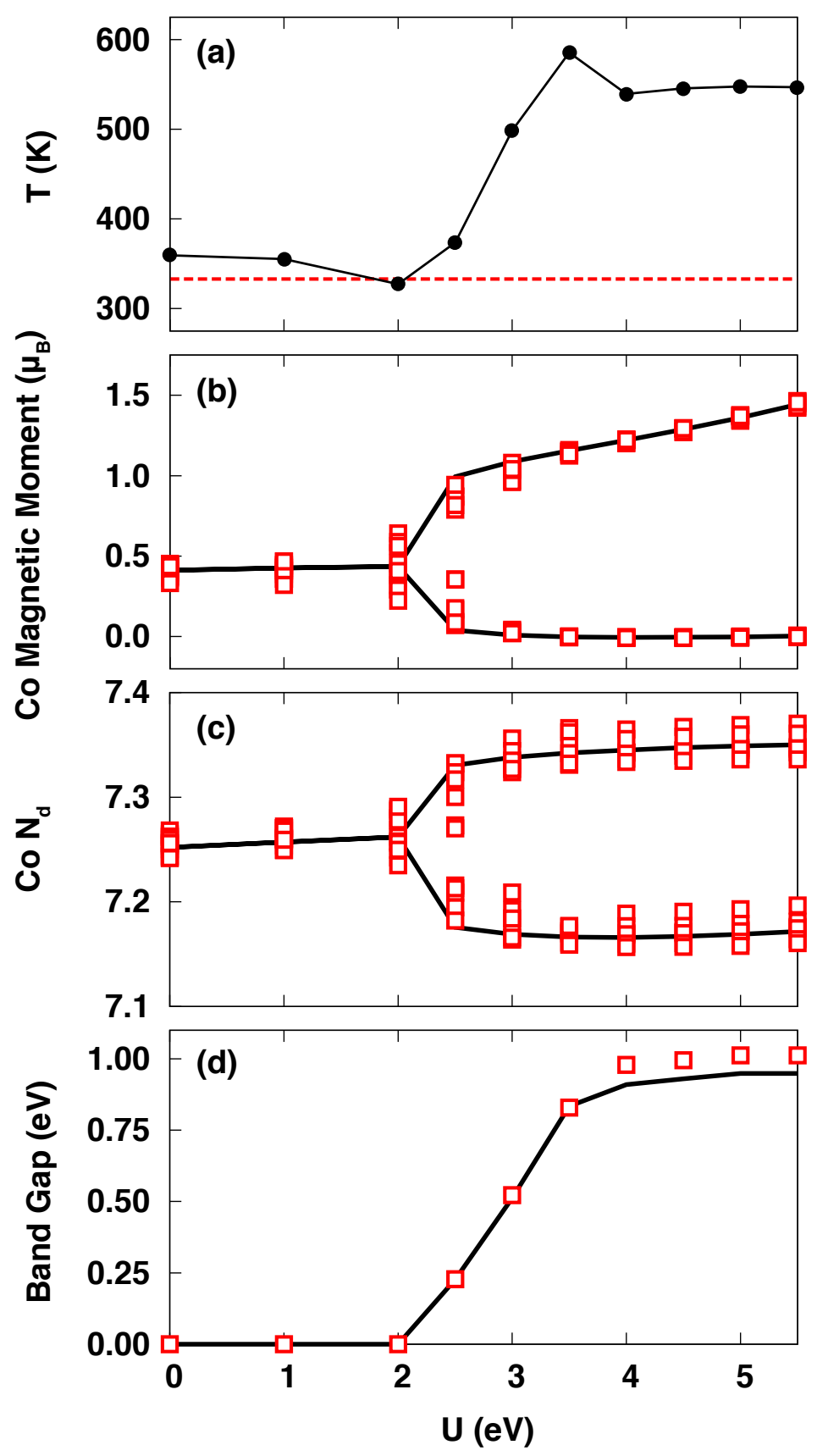

Figure 2.15: (a) Order-disorder transition temperature, (b) Co magnetic moments, (c) Co $\mathrm{N}_{d}$, and (d) band gap for $\mathrm{Li}_{1 / 2} \mathrm{CoO}_{2}$ as a function of $U$ using the frozen $U=0 \mathrm{eV}$ structures. The disordered phase is modeled by the optimal 42-ion special quasirandom structure. In panel (a) the horizontal red line indicates the experimental order-disorder transition temperature from Ref. 123. For the other panels black lines (red squares) correspond to results from the ordered (disordered) structure. 
Our predicted $T_{O-D}$ for the optimal 42-ion SQS cell frozen to the relaxed $U=0$ structures is illustrated in Fig. 2.15(a). Here within DFT the temperature is overestimated by around $25 \mathrm{~K}$ with respect to the experimental value. For small values of $U$ the temperature decreases by as much as $32 \mathrm{~K}$, bringing the prediction closer to the experimental value. For $U>2 \mathrm{eV}$, however, there is a very rapid increase in the predicted transition temperature to values of around 500-600 K, nearly a factor of two greater than the experimental value.

The Co magnetic moments and $N_{d}$ values as a function of $U$ are shown in panels (b) and (c) of Fig. 2.15, respectively. For small $U$ there are small deviations of the magnetic moments and $N_{d}$ compared to those of the ordered structure due to the different local environments of the $\mathrm{Co}$ in the disordered phase. The range of these values are enhanced for $U=2 \mathrm{eV}$, above which both the ordered and disordered cells charge order and open an electronic band gap [see panel $(\mathrm{d})]$. After the CO transition, there is still some spread in the magnetic moment and $N_{d}$ values for the disordered phase with respect to those of the ordered phase.

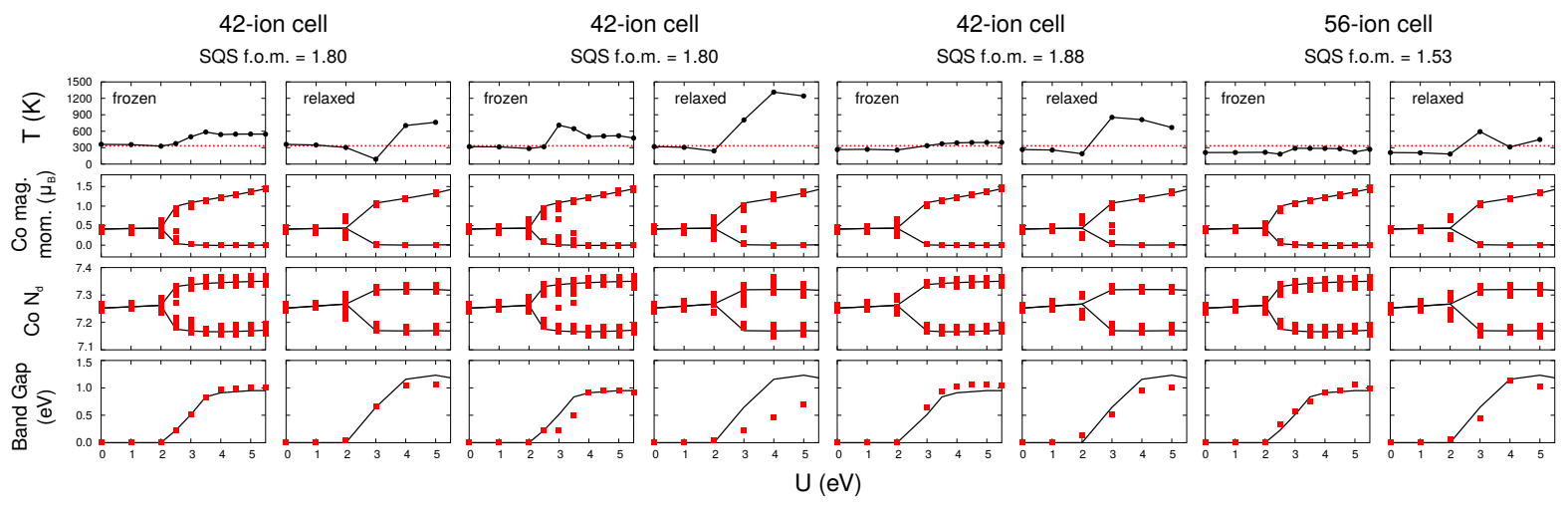

Figure 2.16: Order-disorder transition temperature and Co magnetic moment, Co $N_{d}$, and band gap for ordered and disordered structures as a function of $U$. For each of the four SQS cells considered, the results without (with) structure relaxations are shown to the left (right). For the Co magnetic moment, Co $N_{d}$, and band gap, black lines and filled circles correspond to the ordered structure and red squares correspond to the disordered structure. The figure of merit (f.o.m.) for each SQS structure is indicated.

This demonstrates that the CO transition found within the DFT $+U$ approach is responsi- 
ble for the severe overestimation of the order-disorder transition temperature for $\mathrm{Li}_{1 / 2} \mathrm{CoO}_{2}$. In Fig. 2.16, we show results for additional SQS cells with and without structural relaxations. We find that within DFT $T_{O-D}$ can be overestimated or underestimated depending on the particular cell. A substantial increase in $T_{O-D}$ due to $\mathrm{CO}$ is often observed, though it is not always as dramatic this particular case and does not always lead to as large a disagreement with the experimental value. In general, the results indicate that $\mathrm{DFT}+U$ is highly unreliable in predicting the order-disorder transition temperature due to CO. This suggests that $\mathrm{CO}$ is an artifact of the approximate nature of the interaction in $\mathrm{DFT}+U$ as discussed in Sec. 2.4.3, which is consistent with the lack of evidence for static CO in nuclear magnetic resonance experiments for $\mathrm{Li}_{x} \mathrm{CoO}_{2}$ [Ménétrier et al. (1999)]. We note that other spectroscopic studies that claim CO are likely observing many-body fluctuations rather than static CO in the electronic state of $\mathrm{Li}_{x} \mathrm{CoO}_{2}$ of intermediate $x$ [Mizokawa et al. (2013)] Since $\mathrm{DFT}+U$ often drives $\mathrm{CO}$, this suggests that that $\mathrm{CO}$ predicted by $\mathrm{DFT}+U$ might be artificial in a more general class of materials.

It should be noted that the spurious behavior we have documented in the context of this SQS calculation of $T_{O-D}$ would likely manifest itself in any parameterization of the cluster expansion. In the best case scenario, this could lead to a complicated cluster expansion with long range interactions. The entire procedure could become ill-posed in the worst case with numerous orbitally ordered and/or CO states close in energy for a given Li ordering). This behavior does not bode well for the application of DFT $+U$ in alloy thermodynamics.

\subsection{Conclusions}

At the $\mathrm{DFT}+U$ level of theory, electronic correlations tend to destabilize intermediate- $x$ compounds of both phase stable $\mathrm{Li}_{x} \mathrm{CoO}_{2}$ and phase separating $\mathrm{Li}_{x} \mathrm{FePO}_{4}$. A new formation energy decomposition, which disentangles the distinct roles of the filling and ordering of $d$ 
orbitals, reveals that reduced orbital ordering in compounds of intermediate Li concentration is responsible for this effect. For intermediate $x \mathrm{DFT}+U$ predicts charge ordering which opens electronic band gaps and dampens, though does not eliminate, the tendency for phase separation. Structural relaxations, which similarly only reduce the magnitude of this destabilization, have a more significant impact on $\mathrm{Li}_{x} \mathrm{FePO}_{4}$ than $\mathrm{Li}_{x} \mathrm{CoO}_{2}$ due to the stronger electron-lattice coupling. The same formation energy behavior is observed in $\mathrm{Li}_{x} \mathrm{CoPO}_{4}$, for which a stable intermediate- $x$ compound exists unlike isostructural $\mathrm{Li}_{x} \mathrm{FePO}_{4}$.

The order-disorder transition temperature of $\mathrm{Li}_{1 / 2} \mathrm{CoO}_{2}$ is severely overestimated within $\mathrm{DFT}+U$ as a result of charge ordering. Along with a lack of experimental evidence, this suggests static charge ordering is likely an unphysical artifact of the method. It is found that the approximate treatment of the Coulomb interaction within $\mathrm{DFT}+U$, rather than the double counting correction, is responsible for this behavior. Therefore, future work should focus on improved solutions to the interaction problem in realistic correlated battery cathode materials using dynamical mean-field theory or other approaches.

\subsection{Acknowledgments}

This research used resources of the National Energy Research Scientific Computing Center, a DOE Office of Science User Facility supported by the Office of Science of the U.S. Department of Energy under Contract No. DE-AC02-05CH11231. E.B.I. gratefully acknowledges support from the U.S. Department of Energy Computational Science Graduate Fellowship (Grant No. DE-FG02-97ER25308). 
3. Density functional plus dynamical mean-field theory of $\mathrm{Li}_{x} \mathrm{CoO}_{2}$

\section{Chapter 3}

\section{Density functional plus dynamical mean-field theory of $\mathrm{Li}_{x} \mathrm{CoO}_{2}$}

Transition metal oxides may exhibit strong electronic correlations and are a challenge for firstprinciples electronic structure calculations. Here we employ the density functional theory plus dynamical mean-field theory (DFT+DMFT) approach to study $\mathrm{Li}_{x} \mathrm{CoO}_{2}$, the prototypical rechargeable battery cathode material, at the fully lithiated and delithiated compositions. We solve the single-site, paramagnetic DMFT equations using the continuous-time quantum Monte Carlo solver as well as the Hartree-Fock solver $(\mathrm{DFT}+U)$, and when possible compare results for projector versus maximally-localized Wannier function correlated subspace. Local interactions strongly impact the energetics of the band insulator $\mathrm{LiCoO}_{2}$, most significantly via the $E_{g}$ orbitals, which are partially occupied via hybridization with $\mathrm{O} p$ states. We find $\mathrm{CoO}_{2}$ to be a moderately correlated Fermi liquid with a quasiparticle weight of 0.6-0.8; here the interactions impact the $T_{2 g}$ states the most. In both systems, there are appreciable dynamical charge and spin fluctuations. As compared to the static $(\mathrm{DFT}+U)$ method, DFT+DMFT tends to considerably dampen the increase in total energy as $U$ is increased, and leads to more modest changes in the number of $d$ electrons $\left(N_{d}\right)$ as a function of $U$ (when excluding full charge self-consistency). The average intercalation voltage predicted by DFT + DMFT differs substantially from that of $\mathrm{DFT}+U$, which indicates that dynamical correlations are important to describe this class of materials. Possible reasons for an underprediction of the experimental $\mathrm{Li}_{x} \mathrm{CoO}_{2}$ voltage within DFT+DMFT are discussed. 


\subsection{Introduction}

Strongly correlated materials, for which density functional theory (DFT) calculations often break down due to strong electron-electron interactions, are a challenging class of condensed matter systems relevant to several important technologies [Kotliar and Vollhardt (2004); Morosan et al. (2012)]. One example is Li ion rechargeable batteries. These electrochemical cells rely critically on a cathode material that can reversibly intercalate Li ions [Whittingham (2004)]. Since cathode materials typically are based on transition metal oxides to accommodate changes in oxidation state, they have an open $d$ electron shell and are susceptible to strong correlation physics.

Currently the dominant cathode material is based on $\mathrm{Li}_{x} \mathrm{CoO}_{2}$ (LCO), a layered compound in which Li ions are intercalated between layers of edge-sharing $\mathrm{Co}-\mathrm{O}$ octahedra as shown in Fig. 3.1(c) [Mizushima et al. (1980)]. Several early theoretical studies that revealed significant insight into the electronic structure and phase diagram of LCO [Czyżyk et al. (1992); Aydinol et al. (1997); Wolverton and Zunger (1998); Van der Ven et al. (1998)] were based on DFT [Hohenberg and Kohn (1964); Kohn and Sham (1965)], the de facto standard for first-principles calculations in solid-state physics and chemistry. It is not uncommon, however, for DFT to fail to capture the physics of correlated materials due to the approximation for the exchange-correlation functional [e.g. local density approximation (LDA) or generalized gradient approximation (GGA)].

While DFT in many ways reliably characterizes LCO, there are deficiencies in its description. DFT underestimates the voltage by around $0.8 \mathrm{~V}$ [Chevrier et al. (2010)] and overestimates the order-disorder transition temperature for $x=1 / 2$ by $100{ }^{\circ} \mathrm{C}$ [Van der Ven et al. (1998)], for example. One widely utilized approach to go beyond DFT is the DFT $+U$ method [Liechtenstein et al. (1995)], in which an explicit on-site Coulomb interaction $U$ is added to describe strong correlations in the $d$ shell along with a simple mean-field ansatz for the energy functional, does not fully remedy these issues and in cases hurts the description more than it helps. DFT $+U$ still underestimates the voltage by $0.3 \mathrm{~V}$, and it can overesti- 
mate the order-disorder transition temperature by as much as several hundred degrees [see Chap. 2]. This method drives $\mathrm{LiCoO}_{2}$ towards a high-spin transition [Andriyevsky et al. (2014)] not observed in experiments [van Elp et al. (1991); Ménétrier et al. (1999, 2008)] and, unless spurious charge ordering is permitted to occur, incorrectly predicts phase separation [see Chap. 2 and also Ref. 130]. Moreover, $\mathrm{DFT}+U$ finds $\mathrm{CoO}_{2}$ to be an insulator in disagreement with experiment [Zhang et al. (2004)]. DFT $+U$ clearly is problematic in the context of LCO.

Here we revisit the electronic structure and voltage of LCO using more sophisticated DFT plus dynamical mean-field theory (DFT+DMFT) calculations [Kotliar et al. (2006)]. In this framework, the many-body DMFT approach captures the dynamical local correlations of Co $d$ electrons embedded in the crystal, whereas only the static correlations are described within $\mathrm{DFT}+U$. We find that $\mathrm{DFT}+\mathrm{DMFT}$ describes $\mathrm{LiCoO}_{2}$ as a band insulator with modest shifts and broadenings of the low-energy spectrum, most prominently via the $E_{g}$ levels partially occupied via hybridization with oxygen. $\mathrm{CoO}_{2}$ is a Fermi liquid with quasiparticle weight of around 0.6-0.7, with the $T_{2 g}$ states most strongly affected by the interactions. Dynamical correlations significantly dampen the impact of $U$ on the total energy of both $\mathrm{LiCoO}_{2}$ and $\mathrm{CoO}_{2}$, but more substantially for $\mathrm{CoO}_{2}$, leading to a significantly lower value of the average intercalation voltage as compared to $\mathrm{DFT}+U$. This significant change in the predicted voltage indicate that dynamical correlations, missing in $\mathrm{DFT}+U$, will be necessary for accurate total energy predictions in strongly correlated materials. Finally, we discuss the impact of charge self-consistency, the effect of different choices of the correlated subspace, and possible reasons for the underestimation of the experimental voltage within our DFT+DMFT results. 


\subsection{Computational Details}

We perform single-site paramagnetic DFT+DMFT total energy calculations using the formalism of Ref. 114 based on the spin-independent generalized gradient approximation [Perdew et al. (1996)] and the projector augmented wave method [Blöchl (1994); Kresse and Joubert (1999)] in VASP [Kresse and Hafner (1994, 1993); Kresse and Furthmüller (1996b,a)]. The structures are fixed to the fully relaxed spin-dependent DFT ground state structures with O3 layer stacking [Van der Ven et al. (1998)], corresponding to a band insulator for $x=1$ and a ferromagnetic low-spin metal for $x=0$. Except where otherwise noted, calculations are performed using the fixed non-spin-polarized DFT charge density so they are non-chargeself-consistent (NCSC). A $500 \mathrm{eV}$ energy cutoff and $k$-point mesh of $9 \times 9 \times 9$ for LCO and $19 \times 19 \times 19$ for bulk Li is employed and the ionic forces, stress tensor components, and total energy are converged to $0.01 \mathrm{eV} / \AA, 10^{-3} \mathrm{GPa}$, and $10^{-6} \mathrm{eV}$, respectively. To define the correlated subspace, we utilize the maximally-localized Wannier function (MLWF) basis [Mostofi et al. (2008)] for the full $p$ - $d$ manifold and perform a unitary rotation of the $d$ orbitals to minimize the off-diagonal hoppings [Park et al. (2014)]. The Slater-Kanamori (SK) interaction with $J_{\mathrm{SK}}$ set to $0.7 \mathrm{eV}$ is employed, and we use the numerically exact hybridization expansion continuous-time quantum Monte Carlo (CTQMC) solver for the impurity problem [Haule (2007)] at temperature $T=290 \mathrm{~K}$. For DMFT, we perform calculations (1) using density-density interactions and (2) augmenting the density-density interactions with the off-diagonal $J$ terms within the $E_{g}$ manifold. For comparison, we also perform $\mathrm{DFT}+U$ calculations in the projector basis in VASP (LDAUTYPE $=4$ ) and present all our results in terms

of the $U$ and $J$ corresponding to this interaction model via $U=U_{\mathrm{SK}}-8 J_{\mathrm{SK}} / 5$ and $J=7 J_{\mathrm{SK}} / 5$ [Pavarini et al. (2011)]. We employ the fully-localized-limit form of the double counting. The average intercalation voltage $V$ is computed via $\mathrm{eV}=E(\mathrm{Li})+E\left(\mathrm{CoO}_{2}\right)-E\left(\mathrm{LiCoO}_{2}\right)$, where $e$ is the elementary charge and body-centered-cubic Li is the reference electrode [Aydinol et al. (1997)]. 
3. Density functional plus dynamical mean-field theory of $\mathrm{Li}_{x} \mathrm{CoO}_{2}$

\subsection{Results and Discussion}

\subsubsection{Total energy}

The DFT density of states of $\mathrm{LiCoO}_{2}$ and $\mathrm{CoO}_{2}$ are shown in Fig. 3.1(a) and (b), respectively. In these materials, the ability of the oxygens to relax in the out-of-plane direction slightly distorts the $\mathrm{CoO}_{6}$ octahedra and results in a symmetry lowering with $T_{2 g} \rightarrow A_{1 g}+E_{g}^{\prime}$; however, we will still sometimes refer to this manifold as $T_{2 g}$ for brevity. Within DFT, $\mathrm{LiCoO}_{2}$ is a band insulator with nominally filled $T_{2 g}$ and empty $E_{g}$, whereas $\mathrm{CoO}_{2}$ is metallic with a hole in the $T_{2 g}$ manifold. The density of states from the Wannier basis for the full $p$ - $d$ manifold, shown in the dashed red lines, is identical to that of DFT by construction. The Wannier functions are well localized with values for the spread $\left\langle(\mathbf{r}-\overline{\mathbf{r}})^{2}\right\rangle$ of around 0.42 and $0.45 \AA^{2}$ for the individual Co $d$ orbitals of $\mathrm{CoO}_{2}$ and $\mathrm{LiCoO}_{2}$, respectively.

The total energy of $\mathrm{LiCoO}_{2}$ and $\mathrm{CoO}_{2}$ are shown as a function of $U$ for several methodologies in Fig. 3.2. The vertical dashed lines indicate the values of $U$ computed from first principles via linear response [Zhou et al. (2004a)]. The total energies increase with $U$ as expected, for both $\mathrm{LiCoO}_{2}$ and $\mathrm{CoO}_{2}$. We note that for $U=0$ the total energies within $\mathrm{DFT}+U$ and DFT + DMFT are not equal to those of DFT since we have chosen a fixed finite $J$. The magnitude of the increase in total energy with $U$ is generally greater for $\mathrm{CoO}_{2}$ than $\mathrm{LiCoO}_{2}$, which makes sense since the impact of the on-site interaction is expected to be larger for the system for which $T_{2 g}$ is partially filled (nominally). For NCSC DFT $+U$ in the Wannier correlated subspace, for example, over the full range of $U$ shown the increase in energy of $\mathrm{CoO}_{2}$ is $5.7 \mathrm{eV}$ as compared to only $3.6 \mathrm{eV}$ for $\mathrm{LiCoO}_{2}$. For the same set of calculations using the projector correlated subspace, we find the same trend with energy increases of roughly $4.9 \mathrm{eV}$ for $\mathrm{CoO}_{2}$ and $3.5 \mathrm{eV}$ for $\mathrm{LiCoO}_{2}$. Note that the individual total energies from methods utilizing these different correlated subspaces (projector and Wannier) are not directly comparable.

$\mathrm{LiCoO}_{2}$ is described as a band insulator within all of our $\mathrm{DFT}+U$ results for the range of 


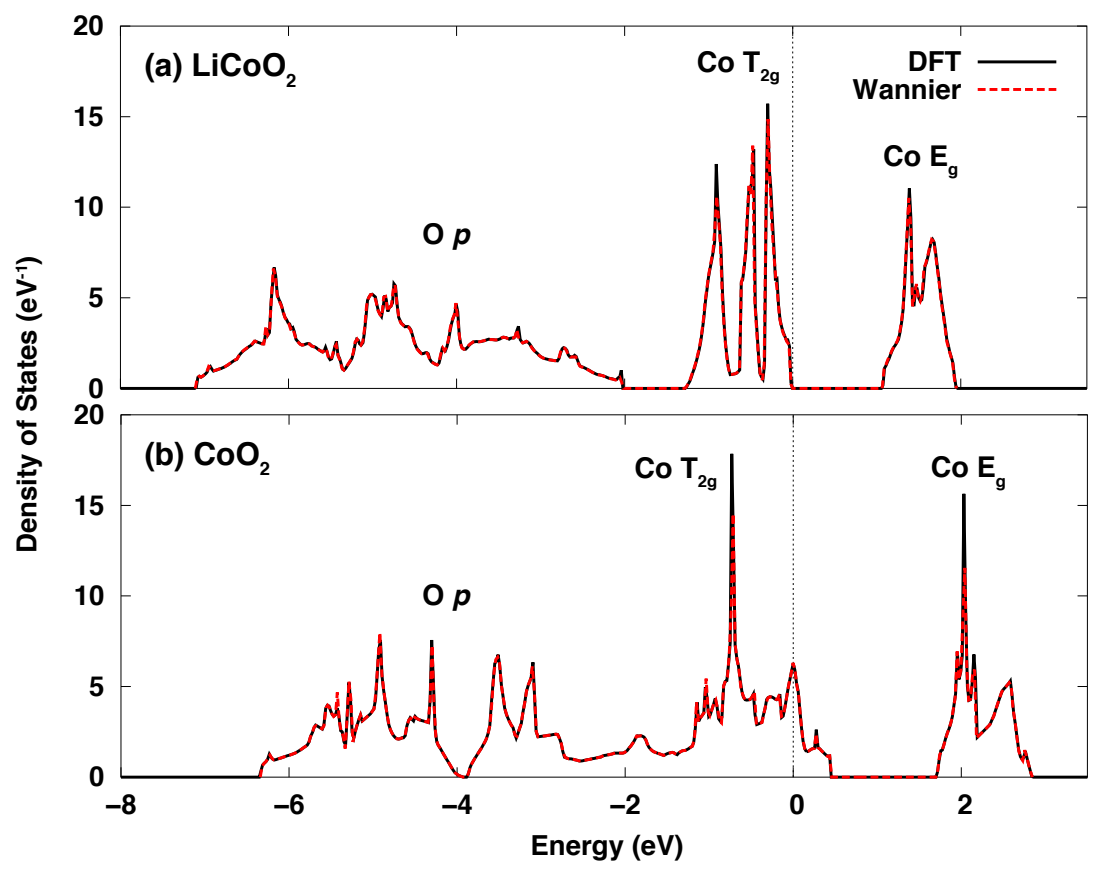

(c) crystal structure
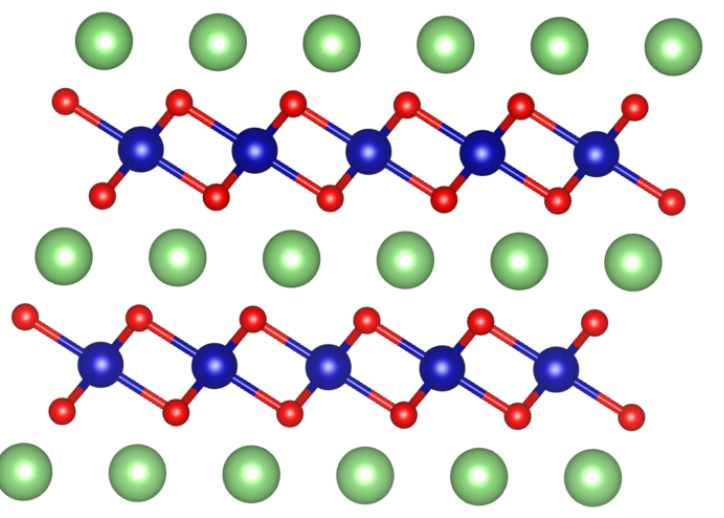

Figure 3.1: Density of states for DFT (black solid lines) and using the Wannier basis (dashed red lines) for (a) band insulating $\mathrm{LiCoO}_{2}$ and (b) metallic $\mathrm{CoO}_{2}$. (c) crystal structure of LCO with $\mathrm{O} 3$ layer stacking with all the Li shown $(x=1)$. The large green, medium blue, and small red spheres represent ionic positions of Li, Co, and $\mathrm{O}$, respectively. The image of the crystal structure is generated using VESTA [Momma and Izumi (2011)]. 
3. Density functional plus dynamical mean-field theory of $\mathrm{Li}_{x} \mathrm{CoO}_{2}$

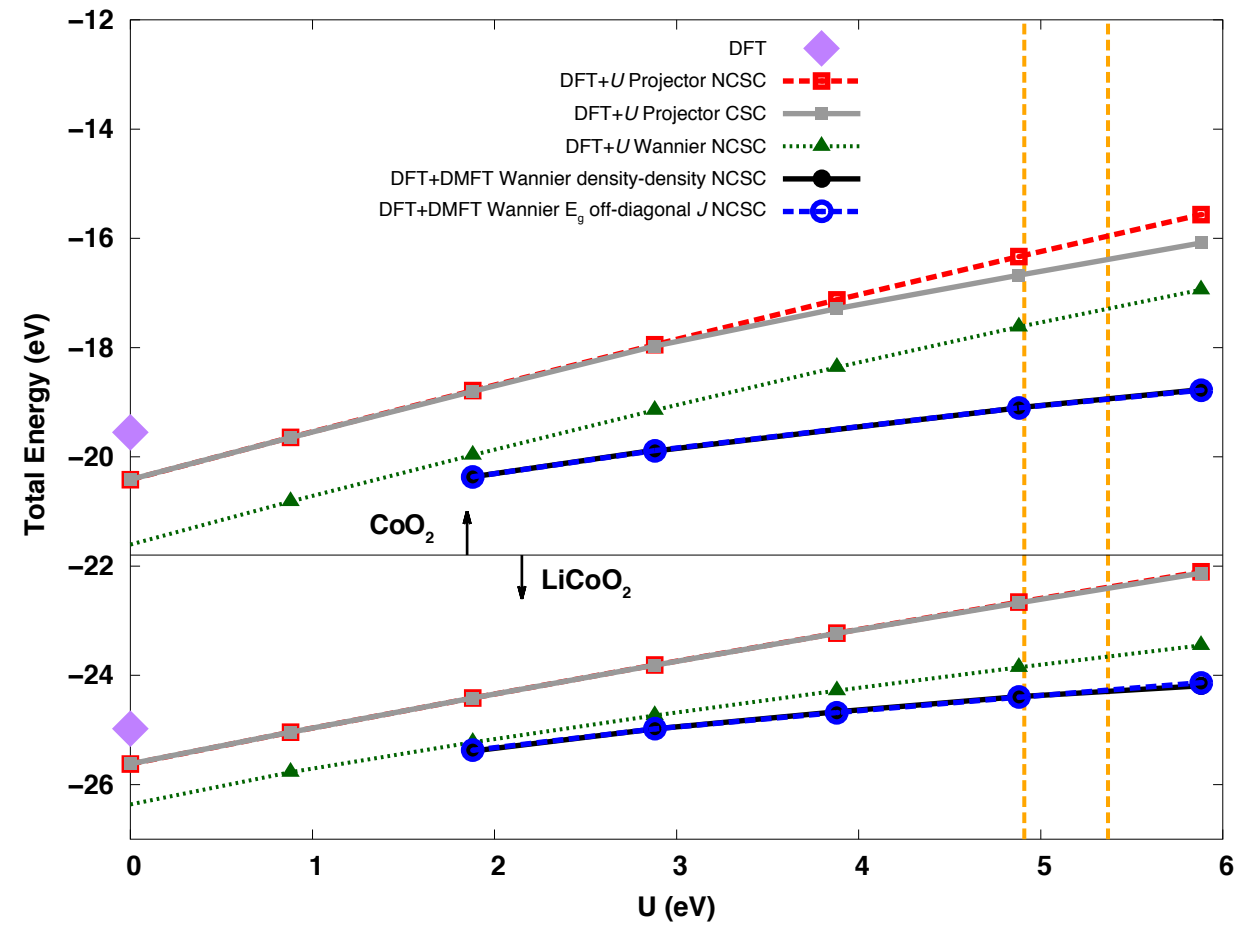

Figure 3.2: Total energy of $\mathrm{CoO}_{2}$ (upper part) and $\mathrm{LiCoO}_{2}$ (lower part) as a function of $U$ for several methodologies including DFT, DFT $+U$, and DFT $+\mathrm{DMFT}$. The dashed orange lines indicate the computed values of $U$ for $\mathrm{LiCoO}_{2}$ (lower value) and $\mathrm{CoO}_{2}$ (higher value) within the linear response approach. 
$U$ shown. In the Wannier correlated subspace, we find $\mathrm{CoO}_{2}$ is a non-spin-polarized metal; only for $U$ above $6.9 \mathrm{eV}$ does $\mathrm{CoO}_{2}$ transition to a magnetic insulator. In contrast, in the projector correlated subspace $\mathrm{CoO}_{2}$ is a spin-polarized metal for smaller values of $U$. This is possibly an artifact of a too-large value of $J$ in this regime of $U$. Above $U=2.9 \mathrm{eV}, \mathrm{CoO}_{2}$ is a non-spin-polarized metal in agreement with the Wannier result. Ultimately, the behavior of the total energy with $U$ is relatively similar for these two choices of correlated subspaces within NCSC DFT $+U$.

For $\mathrm{DFT}+U$ in the projector correlated subspace, we also perform charge-self-consistent (CSC) calculations. Here $\mathrm{LiCoO}_{2}$ is again a band insulator and we find only very small differences (at most $23 \mathrm{meV}$ ) between the NCSC and CSC total energies. $\mathrm{CoO}_{2}$ is a spinpolarized metal for smaller $U$ with differences in total energy of at most $22 \mathrm{meV}$ with respect to the NCSC calculations. However, for $U>2.9 \mathrm{eV} \mathrm{CoO}_{2}$ orbitally orders and opens up a band gap; in this regime the total energies are lowered by several hundreds of meV compared to those of the NCSC calculations. The small impact of changes in charge density on the total energies for $\mathrm{LiCoO}_{2}$ over the full $U$ range and in the metallic phase for $\mathrm{CoO}_{2}$ suggest the fixed charge density may be a reasonable approximation for DFT+DMFT. More importantly, we have a clear guideline on the magnitude of the effect for charge self-consistency within $\mathrm{DFT}+U$, and we expect this is an upper bound for DFT + DMFT calculations in which the impact will likely be dampened.

Within DFT+DMFT, we find very little impact of including the off-diagonal $J$ interaction terms within the $E_{g}$ manifold in addition to the density-density interactions. The magnitude of the differences is typically only around $5-15 \mathrm{meV}$ for $\mathrm{LiCoO}_{2}$ and $3-9 \mathrm{meV}$ for $\mathrm{CoO}_{2}$. This suggests that density-density interactions are likely sufficient to describe this class of systems. In all of the following results, we find no significant difference in employing these two interaction forms. The DFT+DMFT results, which employ the Wannier correlated subspace, appear to merge with the corresponding $\mathrm{DFT}+U$ results in the limit of small $U$, as should be the case. We note again in this limit neither the DFT $+U$ nor the DFT + DMFT 
results converge to the DFT values (large purple diamonds in Fig. 3.2) simply since we take have taken a fixed finite $J$ value whereas the DFT values correspond to $J=0$.

We find the general impact of dynamical correlations on the energetics is to dampen the magnitude of the increase in total energy with $U$ as compared to the static Hartree-Fock treatment in $\mathrm{DFT}+U$. When $U$ is increased from 1.9 to $5.9 \mathrm{eV}$, the total energy of $\mathrm{LiCoO}_{2}$ increases by $2.3 \mathrm{eV}$ within $\mathrm{DFT}+U$ as opposed to only $1.2 \mathrm{eV}$ within DFT+DMFT. For $\mathrm{CoO}_{2}$ the magnitude of these energies is substantially larger with an increase of $3.9 \mathrm{eV}$ for $\mathrm{DFT}+U$ and $1.6 \mathrm{eV}$ for DFT+DMFT. By this measure, dynamical correlations decrease the energy penalty of $U$ by a factor of 2 for $\mathrm{LiCoO}_{2}$ and 2.5 for $\mathrm{CoO}_{2}$. Therefore, dynamical correlations appear to have a larger impact on $\mathrm{CoO}_{2}$ than $\mathrm{LiCoO}_{2}$. This corresponds to very large absolute differences in the energies predicted by DFT $+U$ and DFT + DMFT. For $\mathrm{CoO}_{2}$, for example, around the linear response values of $U$ the difference in energy is around $2 \mathrm{eV}$. This strongly suggests dynamical correlations, missing in the $\mathrm{DFT}+U$ approach, are important for accurate total energies. It should be emphasized that the difference between DFT+DMFT and $\mathrm{DFT}+U$ changes substantially between $\mathrm{LiCoO}_{2}$ and $\mathrm{CoO}_{2}$, and this error will therefore strongly affect observables; this point will be addressed in detail in our discussion of battery voltages below.

\subsubsection{Electronic properties}

The electronic self-energy $\Sigma$ on the imaginary (Matsubara) frequency axis obtained via the CTQMC solver is shown for $\mathrm{CoO}_{2}$ and $\mathrm{LiCoO}_{2}$ in Fig. 3.3 for density-density interactions. The corresponding plots including off-diagonal $J$ in the $E_{g}$ manifold, which show essentially the same behavior, are shown in Fig. 3.4. The noise in the self-energy stems from the stochastic nature of the CTQMC solver, and for frequencies above $20 \mathrm{eV}$ there is no noise since we utilize the analytic form of $\Sigma$ in the high-frequency limit. We note that the selfenergy is sufficiently converged, particularly for low frequency. 


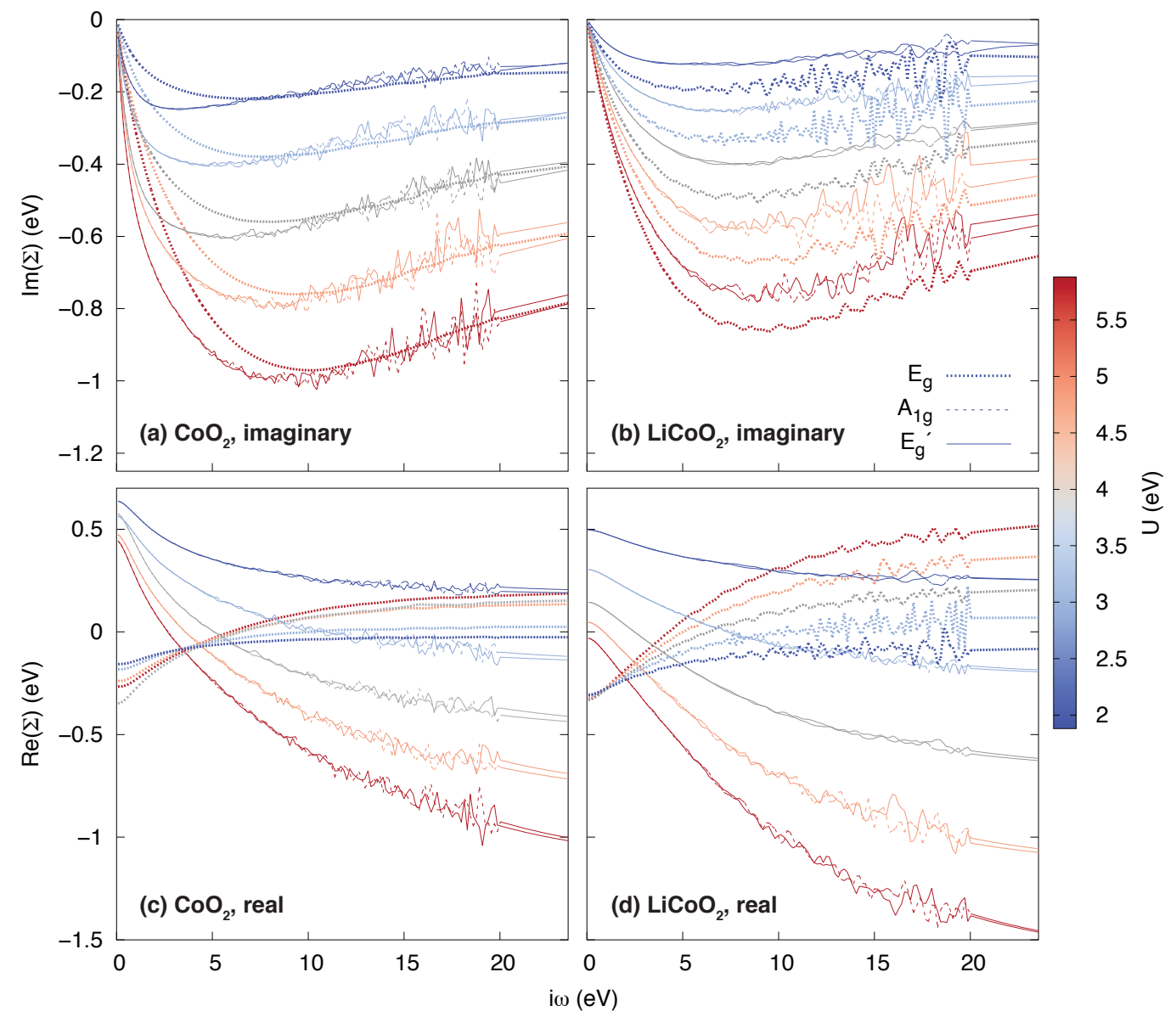

Figure 3.3: Imaginary part of the DMFT self-energy on the imaginary frequency axis for (a) $\mathrm{CoO}_{2}$ and (b) $\mathrm{LiCoO}_{2}$ with density-density interactions for different values of $U$. Solid, dashed, and dotted lines correspond to the $E_{g}^{\prime}, A_{1 g}$, and $E_{g}$ orbitals. (c) and (d) show the corresponding real parts referenced to the chemical potential. 

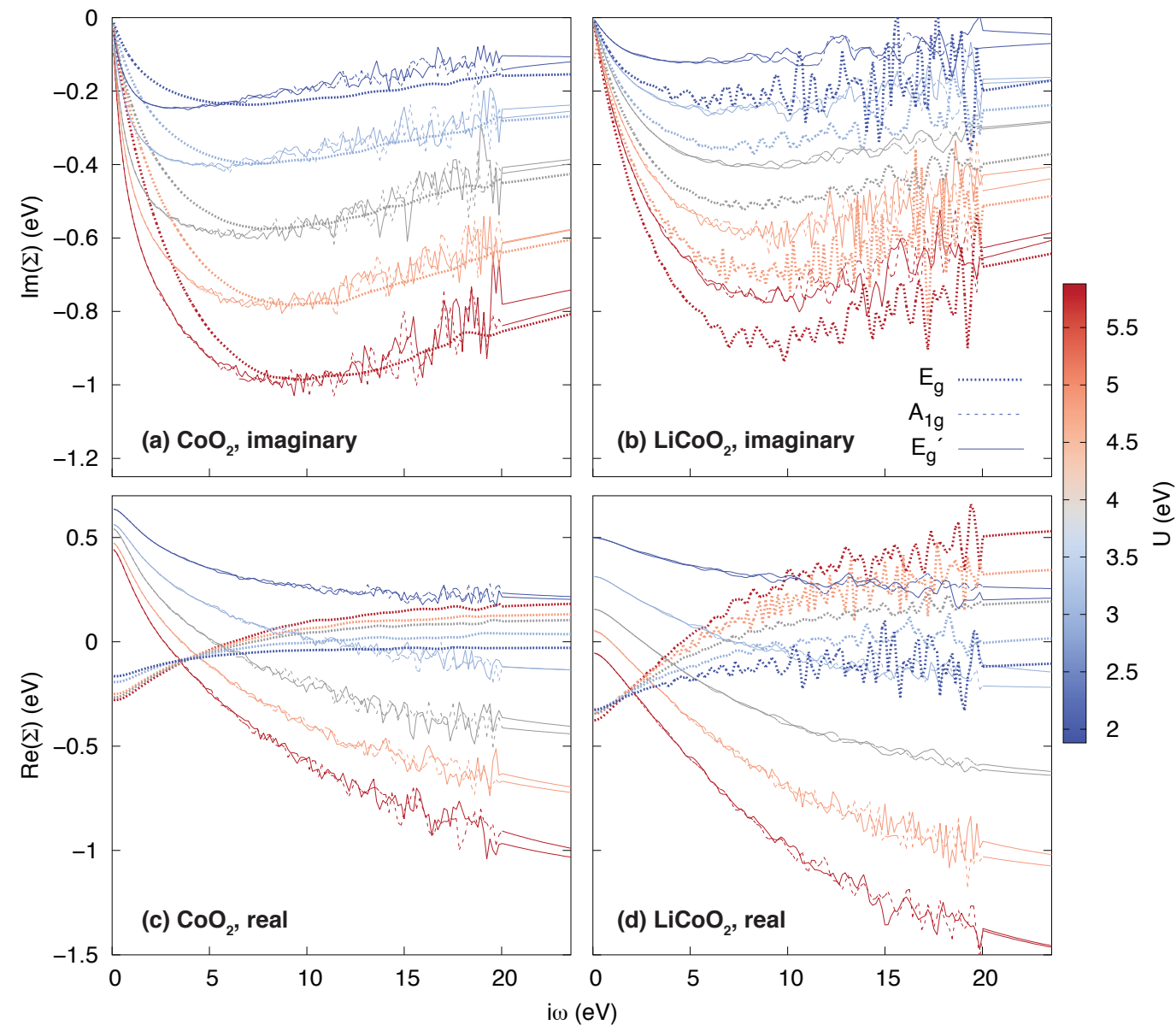

Figure 3.4: Imaginary part of the DMFT self-energy on the imaginary frequency axis for (a) $\mathrm{CoO}_{2}$ and (b) $\mathrm{LiCoO}_{2}$ including off-diagonal $J$ within the $E_{g}$ manifold for different values of $U$. Solid, dashed, and dotted lines correspond to the $E_{g}^{\prime}, A_{1 g}$, and $E_{g}$ orbitals. (c) and (d) show the corresponding real parts referenced to the chemical potential. 
For both $\mathrm{CoO}_{2}$ and $\mathrm{LiCoO}_{2}, \operatorname{Im}(\Sigma)$ goes to 0 at low frequency, which indicates that the $d$ states are well-defined quasiparticles. This is expected for the band insulator $\mathrm{LiCoO}_{2}$ and indicates that $\mathrm{CoO}_{2}$ can be described as a Fermi liquid and is not a Mott insulator. This description agrees with experiments on $\mathrm{CoO}_{2}$ [de Vaulx et al. (2007); Motohashi et al. (2007); Kawasaki et al. (2009)], whereas past DFT $+U$ studies incorrectly predict an insulating state [Zhang et al. (2004)]. Therefore, DFT+DMFT is providing an improved description of the electronic structure of LCO. As a function of $U$, the magnitude of $\operatorname{Im}(\Sigma)$ increases. The imaginary part of the self-energy is essentially identical for the $E_{g}^{\prime}$ and $A_{1 g}$ states, which indicates the symmetry breaking within the $T_{2 g}$ manifold is small. The overall magnitude of $\operatorname{Im}(\Sigma)$ is moderately larger for $\mathrm{CoO}_{2}$ than for $\mathrm{LiCoO}_{2}$. For $\mathrm{CoO}_{2}$, the imaginary part of the self-energy of the $E_{g}^{\prime}$ and $A_{1 g}$ states are larger in magnitude than those of the $E_{g}$ states below $i \omega \approx 10 \mathrm{eV}$. The impact of correlations is stronger for these states since $E_{g}^{\prime}$ and $A_{1 g}$ are partially filled. The opposite trend is found for $\mathrm{LiCoO}_{2}$ with a larger magnitude of $\operatorname{Im}(\Sigma)$ for the $E_{g}$ states for the full range of frequency shown. This suggests that for $\mathrm{LiCoO}_{2}$ the correlations have a larger impact on the nominally-unoccupied $E_{g}$ states since they are partially occupied via hybridization with $\mathrm{O} p$ states, whereas the $E_{g}^{\prime}$ and $A_{1 g}$ are basically completely filled.

For $\mathrm{LiCoO}_{2}$, in the high-frequency limit $\operatorname{Re}(\Sigma)$ is typically negative for $E_{g}^{\prime}$ and $A_{1 g}$ and positive for $E_{g}$. This indicates that the static part of the correlations tend to push $E_{g}^{\prime}$ and $A_{1 g}$ down in energy and $E_{g}$ up in energy as is observed using DFT $+U$. The $U=1.9 \mathrm{eV}$ case is an exception as $J$ is likely too large relative to $U$ in this case. The real part of the selfenergy increases at lower frequency for $E_{g}^{\prime}$ and $A_{1 g}$, whereas it decreases for $E_{g}$. This leads to a higher $\operatorname{Re}(\Sigma)$ for $E_{g}^{\prime}$ and $A_{1 g}$ than $E_{g}$ towards zero frequency. Overall the magnitude of the changes in $\operatorname{Re}(\Sigma)$ with $U$ are significantly larger for $E_{g}^{\prime}$ and $A_{1 g}$ than for $E_{g}$.

For $\mathrm{CoO}_{2}$ the self-energy of the $E_{g}$ states have a small real part (at most $0.21 \mathrm{eV}$ ), which decreases and becomes negative at low frequency. The magnitude is substantially larger for $E_{g}^{\prime}$ and $A_{1 g}$ than $E_{g}$ with a maximum magnitude of $1.2 \mathrm{eV}$ for $U=5.9 \mathrm{eV}$. For these states, 
3. Density functional plus dynamical mean-field theory of $\mathrm{Li}_{x} \mathrm{CoO}_{2}$

like in the $\mathrm{LiCoO}_{2}$ case the values are negative at high frequency (except for very low $U$ ) and becomes positive at low frequency. As opposed to the imaginary part, the real part of the self-energy has smaller magnitude for $\mathrm{CoO}_{2}$ than for $\mathrm{LiCoO}_{2}$.

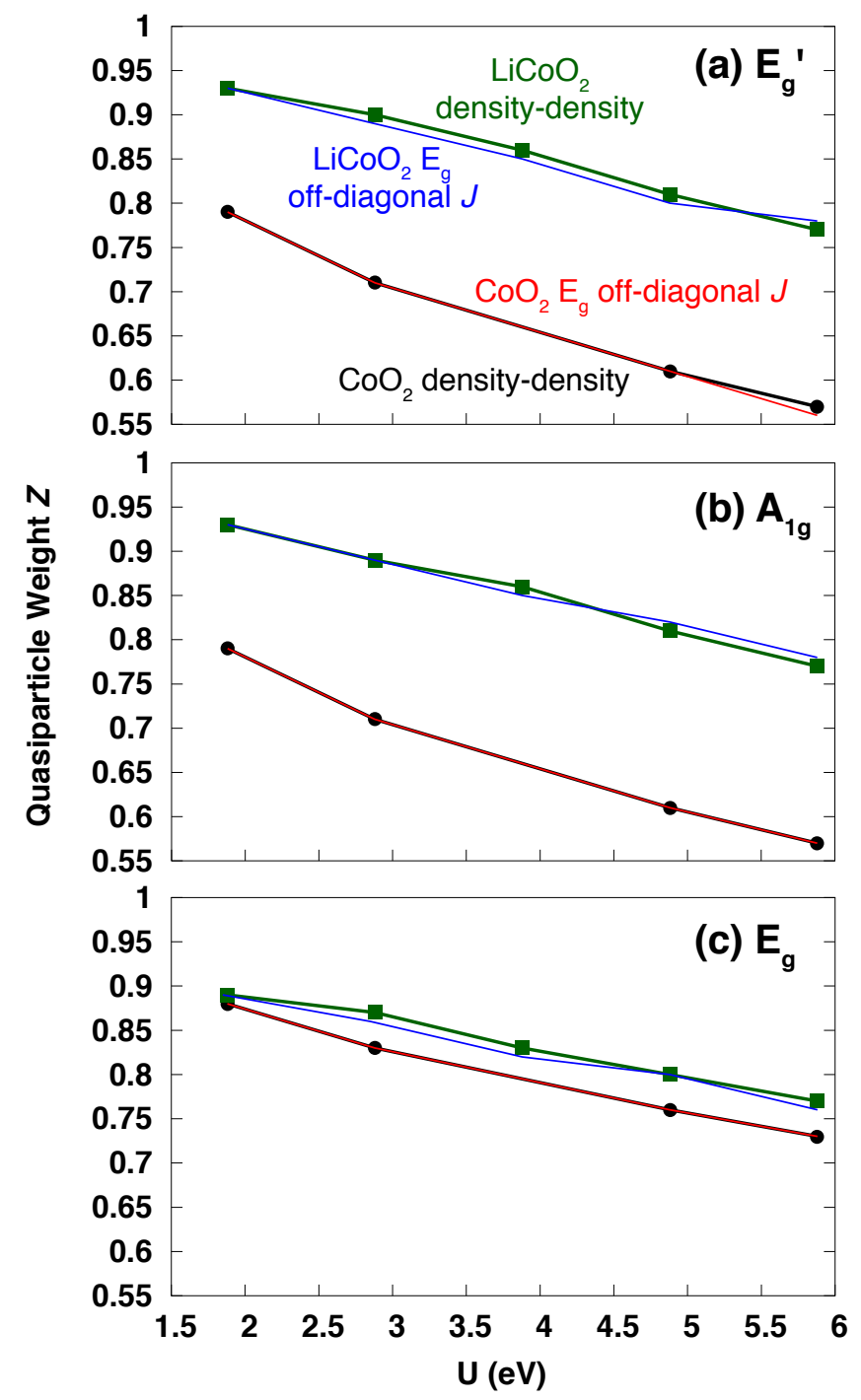

Figure 3.5: Quasiparticle weight $Z$ as a function of $U$ for (a) $E_{g}^{\prime}$, (b) $A_{1 g}$, and (c) $E_{g}$ orbitals.

From the low-frequency behavior of $\operatorname{Im}(\Sigma)$, we compute the quasiparticle weight $Z=$ $\left[1-\partial \operatorname{Im}(\Sigma) /\left.\partial i \omega\right|_{i \omega \rightarrow 0}\right]^{-1}$ shown in Fig. 3.5. This quantity is unity for non-interacting 
electrons $[\operatorname{Im}(\Sigma)=0]$ and is inversely proportional to the effective mass arising from electron interactions. All the values decrease with $U$, as expected, in a roughly linear fashion. $Z$ is always larger for $\mathrm{LiCoO}_{2}$ than $\mathrm{CoO}_{2}$, consistent with the fact that $\mathrm{LiCoO}_{2}$ is a band insulator. This effect is pronounced in the $E_{g}^{\prime}$ and $A_{1 g}$ states, for which the $\mathrm{CoO}_{2}$ values are 0.14-0.20 lower than those of $\mathrm{LiCoO}_{2}$. For the $E_{g}$ states the disparity is smaller with differences of only 0.01-0.04. For $\mathrm{CoO}_{2}, Z$ is larger and decreases less rapidly for the $E_{g}$ orbitals. From $U=1.9$ to $5.9 \mathrm{eV}, Z$ of the $E_{g}^{\prime}$ and $A_{1 g}$ orbitals of $\mathrm{CoO}_{2}$ goes from 0.79 to 0.57 and that of the $E_{g}$ orbitals goes from 0.88 to 0.73 . For $\mathrm{LiCoO}_{2}$, over the same range of $U, Z$ of the $E_{g}^{\prime}$ and $A_{1 g}$ orbitals goes from 0.93 to 0.77 and that of the $E_{g}$ orbitals goes from 0.89 to 0.77 . Here $Z$ is smaller and decreases less rapidly for the $E_{g}$ states such that at $U=5.8 \mathrm{eV} Z$ is the same for all the orbitals.

To further understand the detailed electronic configuration of $\mathrm{CoO}_{2}$ and $\mathrm{LiCoO}_{2}$, in Fig. 3.6 we plot the probabilities of the different atomic configurations sampled by the CTQMC solver in terms of the number of $d$ electrons and the spin projection $S_{z}$. The results for $U=4.9 \mathrm{eV}$ are shown as a representative example. Here we note that the probability distribution is symmetric about $S_{z}=0$ since our DFT + DMFT calculations are paramagnetic (i.e., there is no long-range magnetic order).

Although $\mathrm{CoO}_{2}$ and $\mathrm{LiCoO}_{2}$ are nominally $d^{5}$ and $d^{6}$, respectively, the probability distribution is centered at higher values of $N_{d}$ for both cases due to the appreciable hybridization with $\mathrm{O} p$ states. For example, for $\mathrm{LiCoO}_{2}$ there is substantial time in the Monte Carlo simulation in which an electron from an $\mathrm{O} p$ state has hopped into an $E_{g}$ orbital giving a $d^{7}$ state. There are substantial fluctuations in $N$ as well as $S_{z}$ for the Co site in both systems. For $\mathrm{CoO}_{2}$ the spin fluctuations are moderately larger than in $\mathrm{LiCoO}_{2}$; there is even probability of $S_{z}=3 / 2$ states. We note that these dynamics of the Co site highlight why both DFT and $\mathrm{DFT}+U$ struggle to capture all the physics in this system.

We examine the behavior of $N_{d}$ versus $U$ for all the methodologies employed in this work in Fig. 3.7. Within DFT, one can observe that $N_{d}$ is larger for the projector correlated 
(a) $\mathrm{CoO}_{2}$

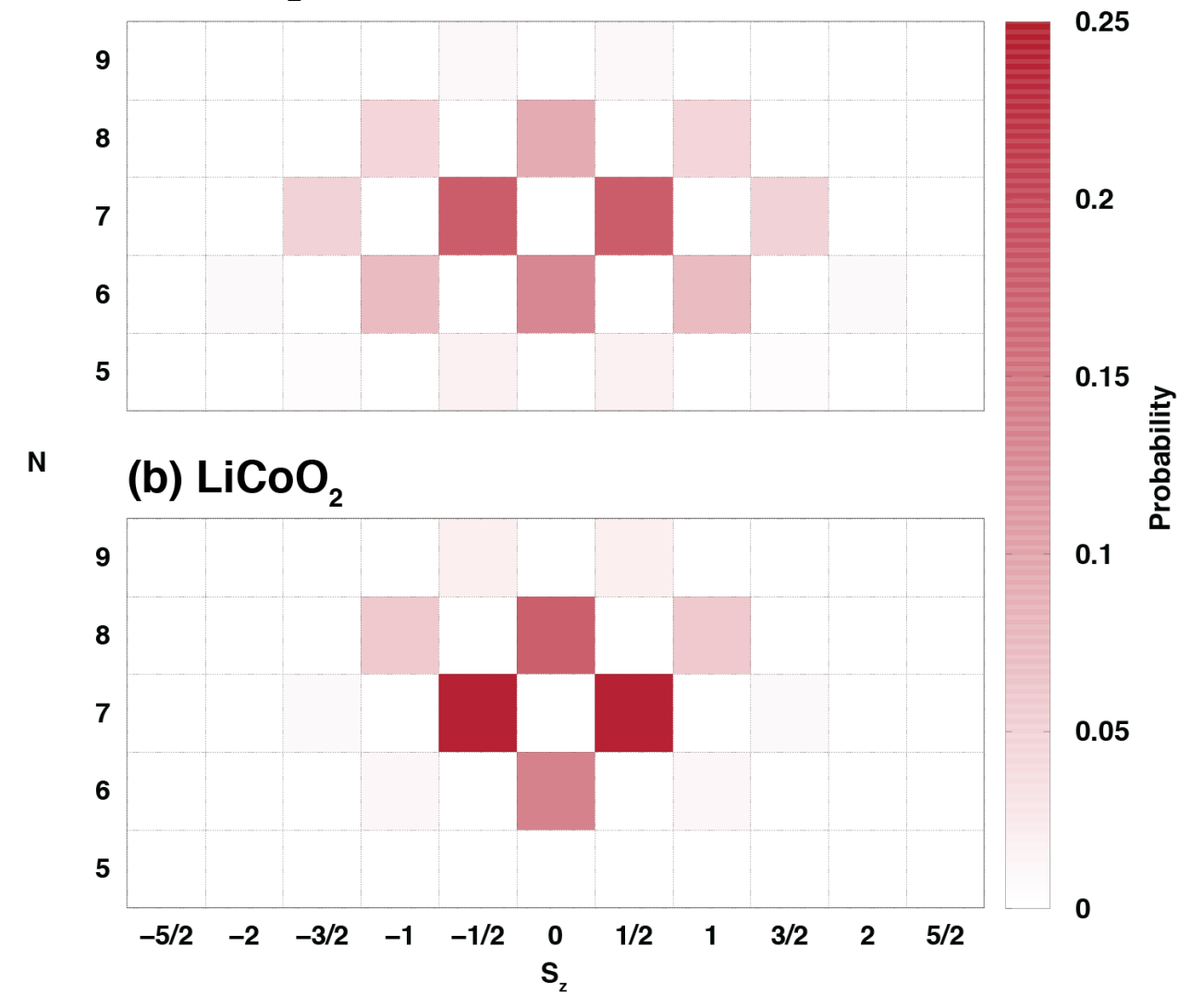

Figure 3.6: Probability of Co atomic states with number of electrons $N$ and spin projection $S_{z}$ for (a) $\mathrm{CoO}_{2}$ and (b) $\mathrm{LiCoO}_{2}$. 
3. Density functional plus dynamical mean-field theory of $\mathrm{Li}_{x} \mathrm{CoO}_{2}$

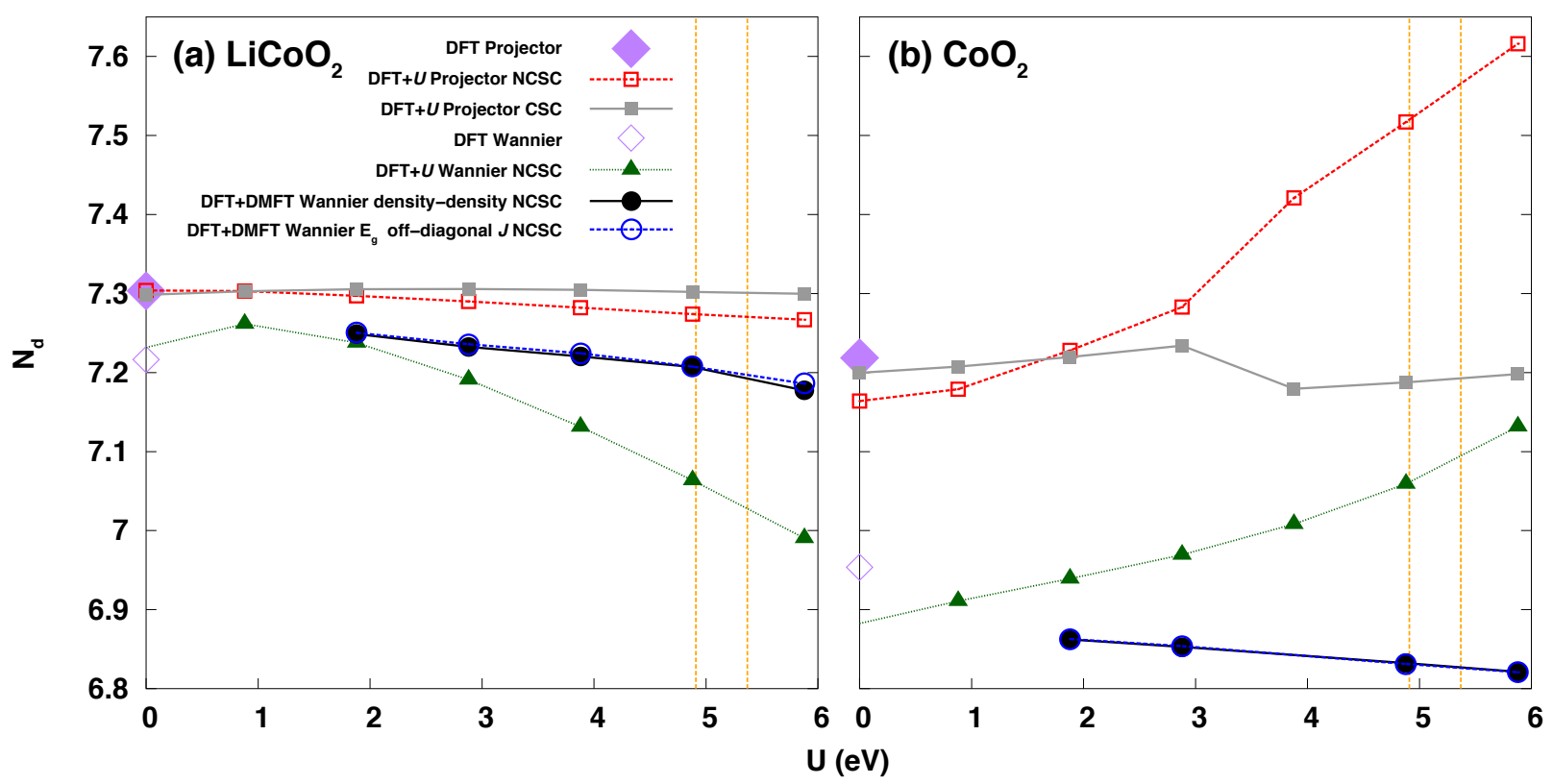

Figure 3.7: $N_{d}$ versus $U$ for (a) $\mathrm{LiCoO}_{2}$ and (b) $\mathrm{CoO}_{2}$ for all the methodologies employed in this study. The dashed orange lines indicate the computed values of $U$ for $\mathrm{LiCoO}_{2}$ (lower value) and $\mathrm{CoO}_{2}$ (higher value) within the linear response approach. 
subspace than the Wannier correlated subspace. The difference is moderate for $\mathrm{LiCoO}_{2}$ (0.09), but significantly larger for $\mathrm{CoO}_{2}(0.26)$. $\mathrm{LiCoO}_{2}$ has $0.08(0.26)$ more electrons than $\mathrm{CoO}_{2}$ in the projector (Wannier) correlated subspace. These values are much smaller than the nominal value of unity, which is indicative of the strong $p-d$ rehybridization in this system [Wolverton and Zunger (1998); Marianetti et al. (2004)].

For $\mathrm{LiCoO}_{2}, N_{d}$ generally decreases with $U$. For DFT $+U$ in the projector correlated subspace, the decrease is small in magnitude (around 0.03 electrons) and including charge self-consistency leads to even smaller changes on the order of 0.006 electrons. In the Wannier correlated subspace, the decrease in $N_{d}$ with $U$ for $\mathrm{DFT}+U$ is more substantial with a change of 0.25 electrons. The inclusion of dynamical correlations (DFT+DMFT) substantially dampens the decrease in $N_{d}$ versus $U$ to around 0.06 electrons.

DFT+DMFT also gives a similar decrease, of 0.04 electrons, in $N_{d}$ of $\mathrm{CoO}_{2}$ with $U$. In contrast, DFT $+U$ show starkly different behavior. Here $N_{d}$ increases dramatically with $U$, by 0.39 electrons for the projector correlated subspace and 0.19 electrons for the Wannier case. This increase in $N_{d}$ is dampened by the metal-insulator transition that occurs using the projector correlated subspace including charge self-consistency, in which case the overall magnitude of the $N_{d}$ variation is only 0.02 electrons. The very large increase in $N_{d}$ for $\mathrm{CoO}_{2}$ within $\mathrm{DFT}+U$ to values even greater than those of $\mathrm{LiCoO}_{2}$ strongly suggests the Hartree-Fock treatment of the impurity problem is breaking down for $\mathrm{CoO}_{2}$. This suggests DFT+DMFT is more reliable to describe $\mathrm{CoO}_{2}$.

The behavior of $N_{d}$ versus $U$ can further be understood by decomposing $N_{d}$ into the components from the $T_{2 g}\left(E_{g}^{\prime}\right.$ and $\left.A_{1 g}\right)$ and $E_{g}$ orbitals, as shown in Fig. 3.8. Within DFT, the Wannier correlated subspace leads to higher (lower) occupancy of $T_{2 g}\left(E_{g}\right)$ by $0.13-0.23$ $(0.32-0.39)$ electrons compared to the projector case. In DFT $+U$, the $\mathrm{LiCoO}_{2} T_{2 g}$ occupancy increases with $U$, whereas the $E_{g}$ occupancy decreases more rapidly; this leads to the overall decrease in $N_{d}$. For the Wannier case the $T_{2 g}$ occupancy increases more rapidly with $U$ at lower $U$ compared to the projector case, and for larger $U$ the occupancy begins to saturate 


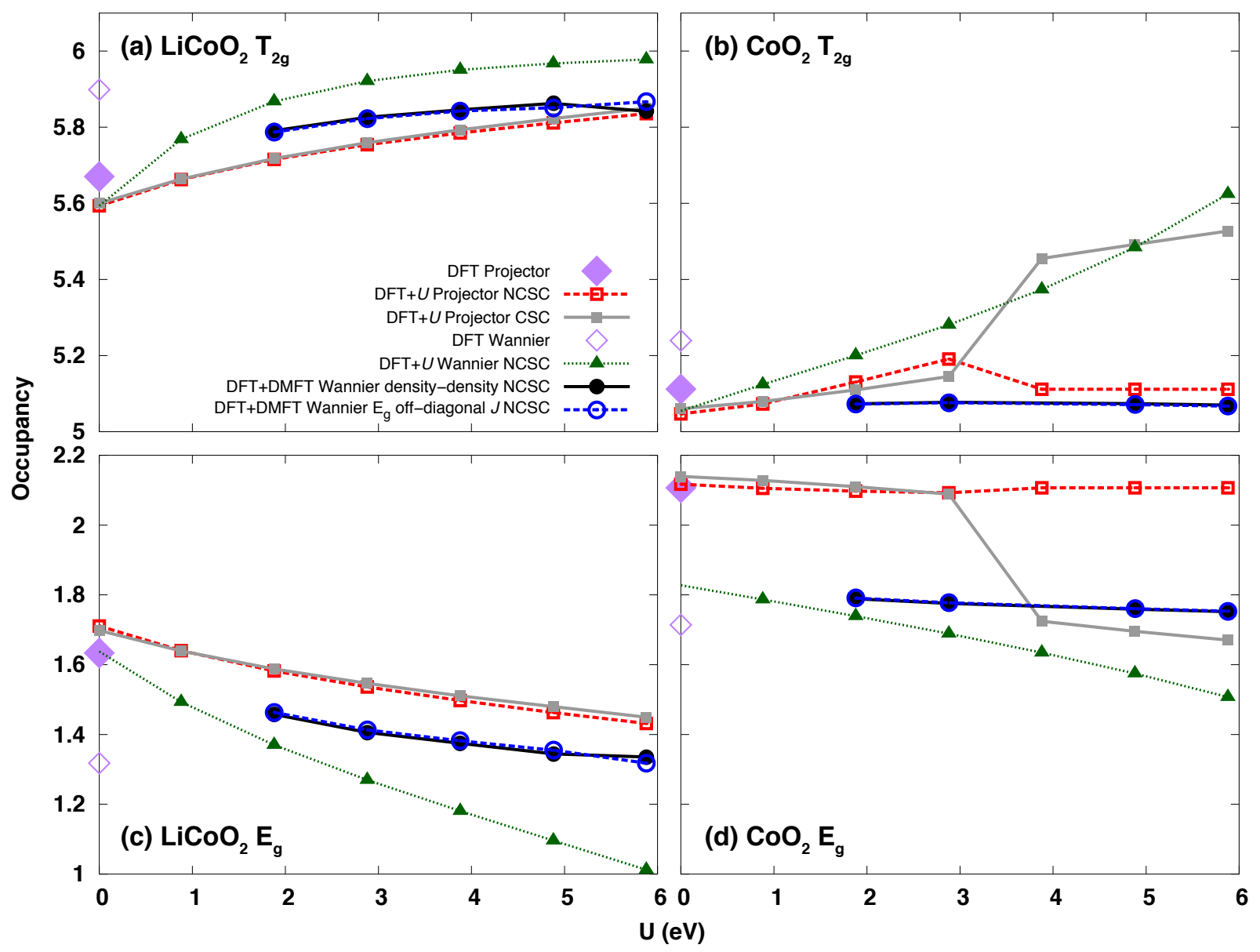

Figure 3.8: Total $T_{2 g}$ occupancy versus $U$ for (a) $\mathrm{LiCoO}_{2}$ and (b) $\mathrm{CoO}_{2}$ for all the methodologies employed in this study. (c) and (d) show the corresponding $E_{g}$ plots. 
close to the nominal value of 6 . Similarly, the decrease in $E_{g}$ occupancy is more substantial in the Wannier case compared to the projector case. Including charge self-consistency has a negligible effect on the occupancies of $\mathrm{LiCoO}_{2}$ in the projector case. The trends in $N_{d}$ are the same for DFT + DMFT as in $\mathrm{DFT}+U$, but the magnitude of the changes in occupancy are much smaller.

Within $\mathrm{DFT}+U$, the $\mathrm{CoO}_{2} T_{2 g}$ occupancy increases substantially by 0.42 electrons with $U$ in the Wannier correlated subspace, whereas the $E_{g}$ occupancy only decreases by 0.23 electrons. Here the $T_{2 g}$ shell is very rapidly moving towards full filling (6) and, to accommodate this, $\mathrm{O} p$ holes are formed. In the projector case, the $T_{2 g}$ occupancy increases by around 0.06 electrons and then decreases once it becomes non-spin-polarized. Overall the changes are much more moderate than those of the Wannier case, in which the $E_{g}$ occupancy is nearly constant with a range of only 0.02 electrons. Including charge self-consistency, once $\mathrm{CoO}_{2}$ becomes insulating the $T_{2 g}$ occupancy sharply increases and the $E_{g}$ occupancy sharply decreases by a larger amount. As in the case of $\mathrm{LiCoO}_{2}$, the changes in occupancy within DFT+DMFT are much smaller than those of NCSC DFT $+U$ with both $T_{2 g}$ and $E_{g}$ occupancies slightly decreasing by 0.003 and 0.04 , respectively, over the range of $U$. Dynamical correlations appear to dampen the changes in $N_{d}$ in the same fashion as charge self-consistency.

\subsubsection{Average intercalation voltage}

We turn our attention to the average intercalation voltage of LCO for $0 \leq x \leq 1$, plotted in Fig. 3.9, which is a key observable for a rechargeable battery cathode. As has been known, DFT tends to underpredict the experimental voltage [Aydinol et al. (1997)], in this case by around $0.7 \mathrm{~V}$. For $\mathrm{DFT}+U$ in the Wannier correlated subspace, the voltage increases roughly linearly with $U$ at a rate of $0.28-0.33 \mathrm{~V}$ per eV. For $U=4.7 \mathrm{eV}$, the voltage agrees with the experimental value of $4.26 \mathrm{~V}$. For the projector correlated subspace, the computed voltage 
3. Density functional plus dynamical mean-field theory of $\mathrm{Li}_{x} \mathrm{CoO}_{2}$

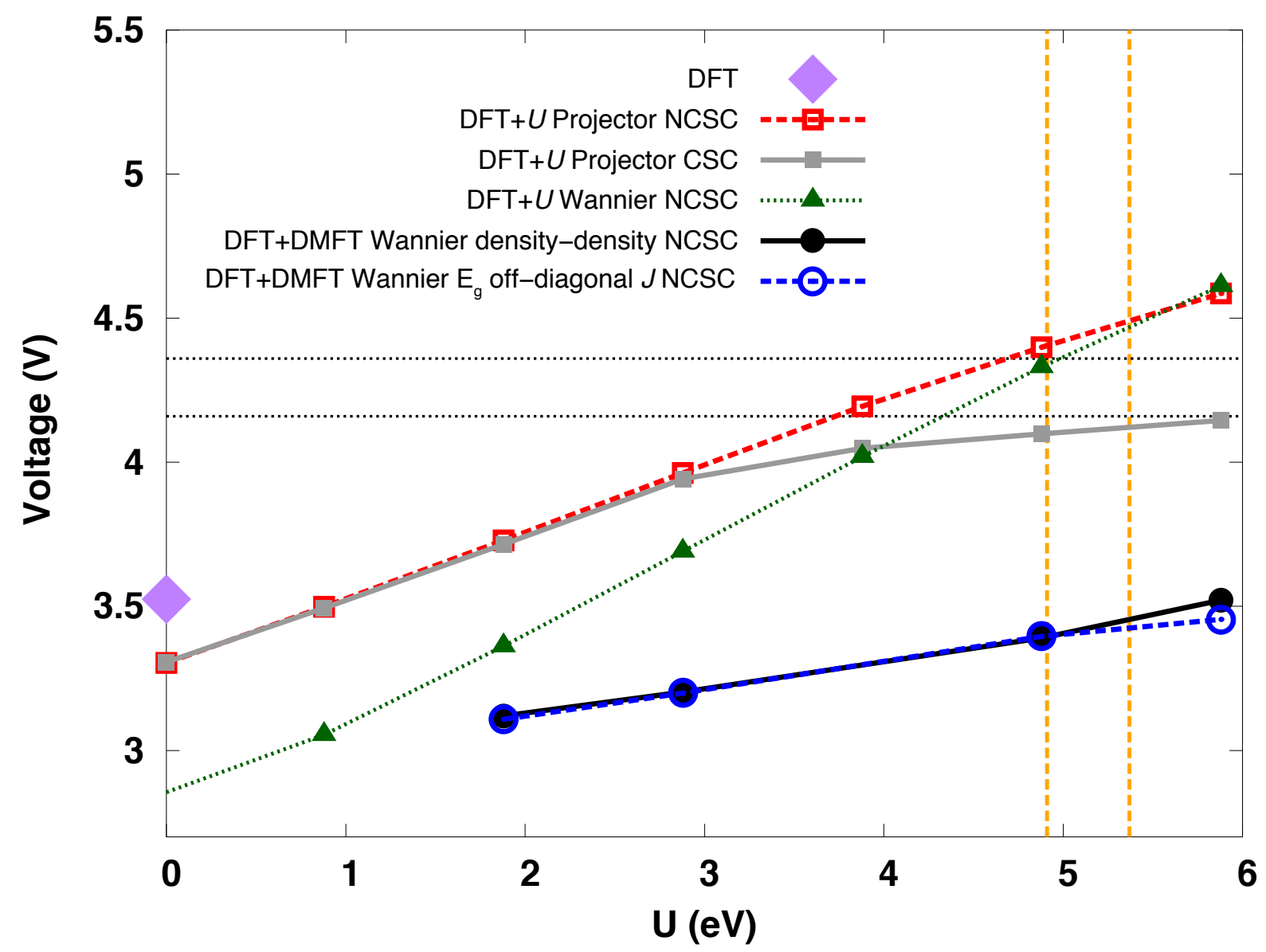

Figure 3.9: Computed intercalation voltage of LCO via $\mathrm{DFT}, \mathrm{DFT}+U$, and $\mathrm{DFT}+\mathrm{DMFT}$ as a function of $U$. The dashed orange lines indicate the computed values of $U$ for $\mathrm{LiCoO}_{2}$ (lower value) and $\mathrm{CoO}_{2}$ (higher value) within the linear response approach. The dotted black lines indicate the expected range of the experimental result [Amatucci et al. (1996)]. 
increases with $U$ at a rate of $0.19-0.23 \mathrm{~V}$ per eV. For $U=4.2 \mathrm{eV}$, in this case, the voltage agrees with experiment. The voltage curve using the Wannier correlated subspace is lower than that of the projector case by a few tenths of an $\mathrm{eV}$ until $U=5.6 \mathrm{eV}$ at which point they intersect. In the projector correlated subspace, including charge self-consistency serves to dampen the increase in voltage with $U$ after $\mathrm{CoO}_{2}$ becomes insulating; in this case a voltage of $4.15 \mathrm{~V}$ is reached by $U=5.9 \mathrm{eV}$. Within DFT+DMFT, the predicted intercalation voltage increases much more slowly as a function of $U$ as compared to $\mathrm{DFT}+U$. Here the changes are around $0.08-0.13 \mathrm{~V}$ per eV. This mainly stems from the dampened increase in energy for $\mathrm{CoO}_{2}$. For the computed values of $U$, the predicted voltage is only 3.39-3.45 V.

The increase in $V$ within $\mathrm{DFT}+U$ and general agreement with experiment was shown previously [Zhou et al. (2004a)] and seemed to suggest that DFT $+U$ is reliable for this class of materials. In DFT+DMFT the quantum impurity problem is solved exactly, whereas in DFT $+U$ a crude, static (Hartree-Fock) approach is employed and dynamical correlations are neglected: DFT + DMFT is superior in every respect. Since the voltage curve predicted by $\mathrm{DFT}+\mathrm{DMFT}$ is substantially different than that of $\mathrm{DFT}+U$, dynamical correlations are essential to describe the energetics of LCO and the closer agreement of DFT $+U$ with experiment is fortuitous.

Our result raises the important question of why there is a substantial disagreement between experiment and DFT+DMFT for the voltage of LCO. We explore several possibilities. Finite temperature is not likely to play a significant role as in both experiment and finitetemperature DFT cluster expansion results the changes in voltage with respect to temperature are only on the order of $10 \mathrm{mV}$ [Wolverton and Zunger (1998); Reynier et al. (2004)]. In addition, we expect the paramagnetic nature of our DFT+DMFT calculations to not be problematic as $\mathrm{LiCoO}_{2}$ and $\mathrm{CoO}_{2}$ do not exhibit long-range magnetic ordering.

It was noted in the original paper computing intercalation voltages from first principles that DFT underestimates the cohesive energy of body-centered-cubic Li [Aydinol et al. (1997)]. Therefore, one should expect an underestimated voltage prediction in our approach 
due to the deficiences of GGA. We note that such an error is not related to the treatment of the correlation problem in the battery cathode material. Within the particular flavor of GGA we employ, the Li cohesive energy is $-1.90 \mathrm{eV}$ as opposed to $-1.63 \mathrm{eV}$ in experiment [Kittel (1986)]. However, there is still a significant underestimation compared to experiment even if one takes this into account.

The choice of the exchange parameter may be responsible for this remaining difference. We find the predicted voltage increases by approximately $0.37 \mathrm{~V}$ via the use of $J_{S K}=0.5$ $\mathrm{eV}$ instead of $J_{S K}=0.7 \mathrm{eV}$. Using this lower value of $J_{S K}$ and the experimental value of $E(\mathrm{Li})$, the $\mathrm{DFT}+\mathrm{DMFT}$ predicted voltage is closer to experimental agreement (within 0.3 $\mathrm{V})$. This indicates that computing an ab initio value of $J$, in addition to $U$, for LCO will be important future work.

One additional possibility is that, within the DFT part of the energetics, errors related to the approximate exchange-correlation potential are significantly different for the band insulator $\mathrm{LiCoO}_{2}$ and the metal $\mathrm{CoO}_{2}$, such that they do not fully cancel in the expression for $V$. Indeed, it has been shown that such a missing error cancellation can lead to significantly inaccurate formation enthalpies (e.g. by as much as several tenths of an $\mathrm{eV}$ ) for this reason [Stevanović et al. (2012)]. A further possibility is that nonlocal correlations (i.e., momentum dependence of the self-energy) are appreciable and nonlocal extensions to DMFT (cluster DMFT) may be necessary to describe LCO. Our results reopen the question of and motivate further study into what is necessary for accurate prediction of the total energies of battery cathode materials and other strongly correlated electron materials.

\subsection{Conclusions}

The electronic structure and intercalation voltage of $\mathrm{Li}_{x} \mathrm{CoO}_{2}$ have been computed us-

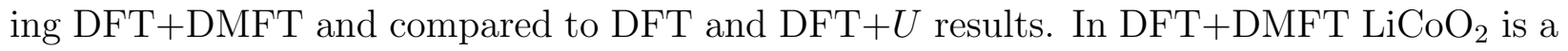
band insulator, while we find that $\mathrm{CoO}_{2}$ is a moderately correlated Fermi liquid in agreement 
3. Density functional plus dynamical mean-field theory of $\mathrm{Li}_{x} \mathrm{CoO}_{2}$

with experiments. Both systems exhibit substantial dynamical charge and spin fluctuations. Dynamical correlations as captured by DFT+DMFT dramatically impact $\mathrm{Li}_{x} \mathrm{CoO}_{2}$ by significantly dampening the changes in total energy and $N_{d}$ found via the DFT $+U$ approach, especially for $\mathrm{CoO}_{2}$. The predicted intercalation voltage within DFT+DMFT differs starkly from that of $\mathrm{DFT}+U$ and suggests that the Hartree-Fock treatment of the impurity problem in $\mathrm{DFT}+U$ is insufficient. DFT $+\mathrm{DMFT}$ underpredicts the average intercalation voltage compared to experiment, raising important questions on what is needed for accurate total energies of strongly correlated electron materials.

\subsection{Acknowledgments}

This research used resources of the National Energy Research Scientific Computing Center, a DOE Office of Science User Facility supported by the Office of Science of the U.S. Department of Energy under Contract No. DE-AC02-05CH11231. E.B.I. gratefully ac-

knowledges the U.S. Department of Energy Computational Science Graduate Fellowship (Grant No. DE-FG02-97ER25308) for support. 
4. Electronic correlations in monolayer $V S_{2}$

\section{Chapter 4}

\section{Electronic correlations in monolayer $\mathrm{VS}_{2}$}

The layered transition metal dichalcogenide vanadium disulfide $\left(\mathrm{VS}_{2}\right)$, which nominally has one electron in the $3 d$ shell, is potent for strong correlation physics and is possibly another realization of an effective one-band model beyond the cuprates. Here monolayer $\mathrm{VS}_{2}$ in both the trigonal prismatic and octahedral phases is investigated using density functional theory plus Hubbard $U(\mathrm{DFT}+U)$ calculations. Trigonal prismatic $\mathrm{VS}_{2}$ has an isolated low-energy band that emerges from a confluence of crystal field splitting and direct $\mathrm{V}-\mathrm{V}$ hopping. Within spin density functional theory, ferromagnetism splits the isolated band of the trigonal prismatic structure, leading to a low-band-gap $S=1 / 2$ ferromagnetic Stoner insulator; the octahedral phase is higher in energy. Including the on-site interaction $U$ increases the band gap, leads to Mott insulating behavior, and for sufficiently high values stabilizes the ferromagnetic octahedral phase. The validity of DFT and DFT $+U$ for these two-dimensional materials with potential for strong electronic correlations is discussed. A clear benchmark is given by examining the experimentally observed charge density wave (CDW) in octahedral $\mathrm{VS}_{2}$, for which DFT grossly overestimates the bond length differences compared to known experiments; the presence of CDWs is also probed for the trigonal prismatic phase. Finally, we investigate why only the octahedral phase has been observed in experiments and discuss the possibility of realizing the trigonal prismatic phase. Our work suggests trigonal prismatic $\mathrm{VS}_{2}$ is a promising candidate for strongly correlated electron

physics that, if realized, could be experimentally probed in an unprecedented fashion due to 
its monolayer nature.

\subsection{Introduction}

Transition metal dichalcogenides (TMDCs), composed of layers of chalcogen-metalchalcogen units (hereafter called monolayers) that stack and adhere via weak bonding, are a diverse class of materials known to exhibit charge density waves, metal-insulator transitions, superconductivity, and novel optoelectronic properties [Wilson and Yoffe (1969)]. Recent breakthroughs in the ability to isolate and manipulate few-layer and monolayer materials, derived from TMDCs like $\mathrm{MoS}_{2}$ and other layered crystals such as graphite, have enabled new possibilities for device applications as well as fundamental studies of low-dimensional systems [Novoselov et al. (2005)].

Many TMDCs are nominally $d^{0}$ (e.g. $\mathrm{TiS}_{2}$ ) or band insulators in which an even number of $d$ electrons completely fills the valence band (e.g. $\mathrm{MoS}_{2}$ ). Such configurations preclude the possibility of strong electronic correlations and/or magnetism in the ground state. However, there are known examples from experiments of non-oxide layered materials exhibiting magnetism and in some cases insulating behavior. Spin-3/2 $\mathrm{Cr} X \mathrm{Te}_{3}$ is a ferromagnetic insulator with Curie temperature of $33 \mathrm{~K}$ for $X=\mathrm{Si}$ and $61 \mathrm{~K}$ for $X=\mathrm{Ge}$; monolayers in this class of materials have been predicted to be stable with ferromagnetic exchange as well [Carteaux et al. (1991, 1995); Lebègue et al. (2013); Li and Yang (2014); Sivadas et al. (2015); Zhuang et al. (2015)]. The spin- $1 / 2$ insulator $\operatorname{Cr} X_{3}$ is a ferromagnet below $37 \mathrm{~K}$ for $X=\mathrm{Br}$ and 61 $\mathrm{K}$ for $X=\mathrm{I}$; in $\mathrm{CrCl}_{3}$ ferromagnetic layers stack in an antiferromagnetic pattern with a Néel temperature of 17 K [de Haas et al. (1940); Tsubokawa (1960); Cable et al. (1961); Dillon Jr. and Olson (1965)]. Ferromagnetic $\mathrm{Fe}_{3} \mathrm{GeTe}_{2}$, which is metallic, has a substantial Curie temperature of $150 \mathrm{~K}$ [Deiseroth et al. (2006); Chen et al. (2013)]. In-plane antiferromagnetism is also observed; $\mathrm{MnPS}_{3}$ and $\mathrm{MnPSe}_{3}$ are spin-5/2 antiferromagnets with Néel temperatures 
of 78 and $74 \mathrm{~K}$, respectively [Wildes et al. (1998); Jeevanandam and Vasudevan (1999)]. Additionally, there are numerous antiferromagnets in the family of Fe pnictide superconductors [Dai (2015)].

$\mathrm{VS}_{2}$ is an interesting candidate among the many possible TMDCs. Here nominal electron counting indicates that $\mathrm{V}$ donates two electrons to each $\mathrm{S}$, leaving it in a $d^{1}$ (i.e., spin$1 / 2$ ) configuration. Therefore, $\mathrm{VS}_{2}$ might be potent for strong electronic correlation physics, especially since its $3 d$ electrons will be significantly more localized than the $4 d$ or $5 d$ electrons of $\mathrm{NbS}_{2}$ or $\mathrm{TaS}_{2}$, respectively. Similarly, the electronic states of the sulfur anion should be more localized than those of selenium or tellurium.

The structure of a monolayer TMDC consists of one metal layer sandwiched between two chalcogen layers with each layer corresponding to a triangular lattice. This gives rise to two basic types of chalcogen-metal-chalcogen stacking: ABA stacking, in which the metal layer hosts a mirror plane, or ABC stacking. The latter gives rise to approximate octahedral coordination of the transition metal (TM) by chalcogens, which results in the five-fold $d$ manifold splitting into a 3 -fold set $\left(T_{2 g}\right)$ and a 2 -fold set $\left(E_{g}\right)$ of orbitals. More precisely, the octahedral environment experiences a trigonal distortion due to the ability of the chalcogens to relax in the out-of-plane direction. This results in a point group symmetry lowering $O_{h} \rightarrow D_{3 d}$ and a further splitting of the $d$ orbitals $T_{2 g} \rightarrow A_{1 g}+E_{g}^{\prime}$. For convenience, we refer to the distorted octahedral $\left(D_{3 d}\right)$ phase as the OCT phase in the remainder of this paper.

Alternatively, ABA stacking results in a trigonal prismatic (TP) coordination of the TM by the chalcogens. The TP coordination, which is compared to that of the OCT structure in Fig. 4.1, splits the $d$ manifold into a one-fold $A_{1}^{\prime}$ orbital and two different types of two-fold orbitals $\left(E^{\prime}\right.$ and $\left.E^{\prime \prime}\right)$. Both OCT and TP coordinations are possible for $\mathrm{VS}_{2}$, and the TP coordination is particularly intriguing since it could potentially be a physical realization of a one-band model with strong interactions; this rare feature is a hallmark of the copper oxide (cuprate) high-temperature superconductors [Zhang and Rice (1988)].

Experimentally the TP phase has not been realized, but bulk $\mathrm{VS}_{2}$ was first synthesized 

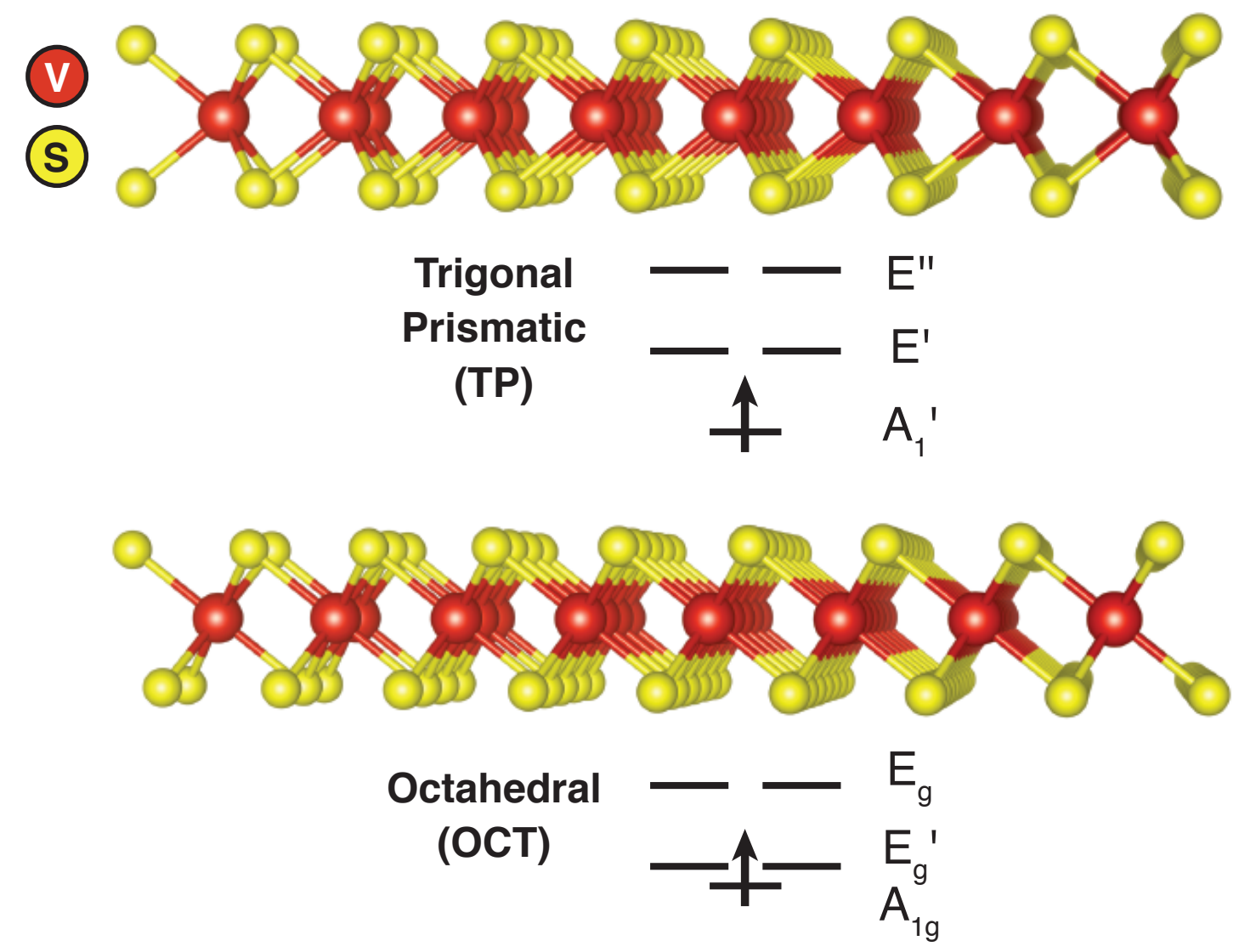

Figure 4.1: Side view of crystal structures of trigonal prismatic and octahedral monolayer $\mathrm{VS}_{2}$ and schematic $\mathrm{V} 3 d$ orbital fillings from crystal field theory. The red and yellow spheres represent ionic positions of $\mathrm{V}$ and $\mathrm{S}$, respectively. 
in the OCT phase in the 1970s by deintercalating LiVS $_{2}$ [Murphy et al. (1977)]. It exhibits a charge density wave $(\mathrm{CDW})$ below $T=305 \mathrm{~K}$ with a wavevector $q \approx 2 / 3 K$, where $K$ is the corner of the Brillouin zone [Murphy et al. (1977); Tsuda et al. (1983); Mulazzi et al. (2010)]. In the CDW phase Mulazzi et al. found metallic resistivity and no lower Hubbard band in the photoemission spectrum, suggesting rather weak electronic correlations [Mulazzi et al. (2010)]. Only a very small paramagnetic response was observed in the magnetic susceptibility, which it was suggested might stem from $\mathrm{V}$ located in between neighboring $\mathrm{VS}_{2}$ monolayers. A more recent high-pressure synthesis by Gauzzi et al. found much more appreciable local magnetic moments but no long-range CDW, and it was speculated that "nm-size domains" might be responsible [Gauzzi et al. (2014)]. Using phonon calculations, they also showed that the presence of a CDW soft mode is very sensitive to the lattice parameters. Nanosheets, though not a monolayer, of $\mathrm{OCT} \mathrm{VS}_{2}$ have been synthesized and interpreted as showing ferromagnetism [Feng et al. (2011, 2012); Gao et al. (2013); Zhong et al. (2014)].

Here we employ first-principles electronic structure calculations based on DFT to explore the physics of $\mathrm{VS}_{2}$. We focus on a single layer of the material since the realization of a strongly correlated monolayer material could enable one to probe Mott physics via gating and strain in an unprecedented way. We find that DFT captures the $q=2 / 3 \mathrm{~K}$ CDW in OCT $\mathrm{VS}_{2}$ and explains the lack of correlations observed experimentally, though it substantially overestimates the structural distortion. The addition of an appreciable on-site Hubbard $U$ interaction to the $\mathrm{V}$ site leads to anti-aligned spins in $\mathrm{OCT} \mathrm{VS}_{2}$ and yields $\mathrm{V}-\mathrm{V}$ distance distortions and metallic behavior in reasonable agreement with known experiments. Unlike the OCT phase, we find that TP $\mathrm{VS}_{2}$ has an isolated low-energy $A_{1}^{\prime}$ band at the level of non-spin-polarized DFT due to the crystal field and direct $\mathrm{V}-\mathrm{V}$ hopping. The preferred magnetic order is ferromagnetic, as opposed to the antiferromagnetic ordering found in the cuprates, and this magnetism opens up a small band gap by splitting the $A_{1}^{\prime}$ band. The onsite interaction leads to a low-band-gap $S=1 / 2$ ferromagnetic Mott insulator. For a narrow 
range of $U$ we find evidence for a CDW in $\mathrm{TP} \mathrm{VS}_{2}$. Although DFT predicts ferromagnetic $\mathrm{TP} \mathrm{VS}_{2}$ is the ground state, for moderate values of $U$ we find the OCT structure becomes thermodynamically favored.

\subsection{Computational Details}

Density functional theory (DFT) [Hohenberg and Kohn (1964); Kohn and Sham (1965)] calculations within the generalized gradient approximation of Perdew, Burke, and Ernzerhof (PBE) [Perdew et al. (1996)] are performed using the Vienna ab initio simulation package (VAsP) [Kresse and Hafner (1994, 1993); Kresse and Furthmüller (1996b,a)]. The Kohn-Sham equations are solved using a plane-wave basis set with a kinetic energy cutoff of $500 \mathrm{eV}$ and the projector augmented wave method [Blöchl (1994); Kresse and Joubert (1999)]. The outof-plane lattice vector length is chosen to be $20 \AA$. To sample reciprocal space we employ a $24 \times 24 \times 1 k$-point grid for the primitive unit cell and $k$-point grids with approximately the same $k$-point density for supercells. We utilize the tetrahedron method with Blöchl corrections [Blöchl et al. (1994)] for all calculations except for structural relaxations and phonon calculations in metals, for which we employ the first-order Methfessel-Paxton method [Methfessel and Paxton (1989)] with a $50 \mathrm{meV}$ smearing. The total energy, ionic forces, and stress tensor components are converged to $10^{-6} \mathrm{eV}, 0.01 \mathrm{eV} / \AA$, and $10^{-3} \mathrm{GPa}$, respectively.

To compute maximally-localized Wannier function (MLWFs) we employ the WANNIER90 code [Mostofi et al. (2008)]. The rotationally-invariant DFT $+U$ approach with fully localized limit double counting [Liechtenstein et al. (1995)] is used to explore the impact of an on-site Hubbard $U$ on the V $3 d$ electrons. Values of on-site Coulomb repulsion $U$ are computed from first principles via the linear response approach of Cococcioni and de Gironcoli [Cococcioni and de Gironcoli (2005)]. We do not employ an on-site exchange interaction $J$ since this effect is present within spin density functional theory [Park et al. (2015)]. We use the direct (supercell) approach in PHONOPY [Togo et al. (2008)] to compute phonon dispersion 
relations. For these calculations we employ a $5 \times 5 \times 1$ supercell for smaller $U$ and a larger $6 \times 6 \times 1$ supercell for $U>3 \mathrm{eV}$, which we find is needed to capture the presence of soft mode instabilities. Phonons at select $q$-points are obtained using the frozen phonon method to assess supercell convergence of direct calculations. Images of crystal structures are generated with VESTA [Momma and Izumi (2011)].

\subsection{Results and Discussion}

\subsubsection{Charge density wave in octahedral $\mathrm{VS}_{2}$ within DFT}

Given that a collection of experiments exist for the bulk OCT phase, we begin by addressing the physics of the OCT monolayer. Since bulk OCT $\mathrm{VS}_{2}$ is known to undergo a CDW transition below $T=305$ K [Murphy et al. (1977); Tsuda et al. (1983); Mulazzi et al. (2010)], we explore the presence of such a CDW in the monolayer OCT structure. We compute the phonon frequencies using the frozen phonon method for $q=2 / 3 K$, the experimental CDW wavevector from electron microscopy [Mulazzi et al. (2010)], and verify the soft mode in the non-spin-polarized (NSP) bulk OCT phase as found in a previous study [Gauzzi et al. (2014)]. We find the frequency is $\omega=60 i \mathrm{~cm}^{-1}$. For the monolayer, at this wavevector we find the same soft mode in the NSP state now with a slightly softer frequency $\omega=80 i \mathrm{~cm}^{-1}$. Given the experimental CDW wavevector is in-plane and the similarity of the soft mode for the bulk and the monolayer, we expect the monolayer CDW to be representative of that of the bulk. Additionally, at a slightly different wavevector of $q=3 / 5 \mathrm{~K}$ we find a soft mode of smaller magnitude $\omega=48 i \mathrm{~cm}^{-1}$ in the monolayer.

Without any CDW the lowest-energy state of monolayer OCT $\mathrm{VS}_{2}$ is a ferromagnetic (FM) metal with a $\mathrm{V}$ magnetic moment of $0.5 \mu_{B}$, which is $13 \mathrm{meV}$ lower in energy than the NSP state. The relaxed NSP $q=2 / 3 \mathrm{~K}$ OCT CDW state is $12 \mathrm{meV}$ lower in energy than the pristine (without-CDW) FM state. Although we find no soft mode for the pristine OCT 
(a) OCT $q=2 / 3 \mathrm{~K}$

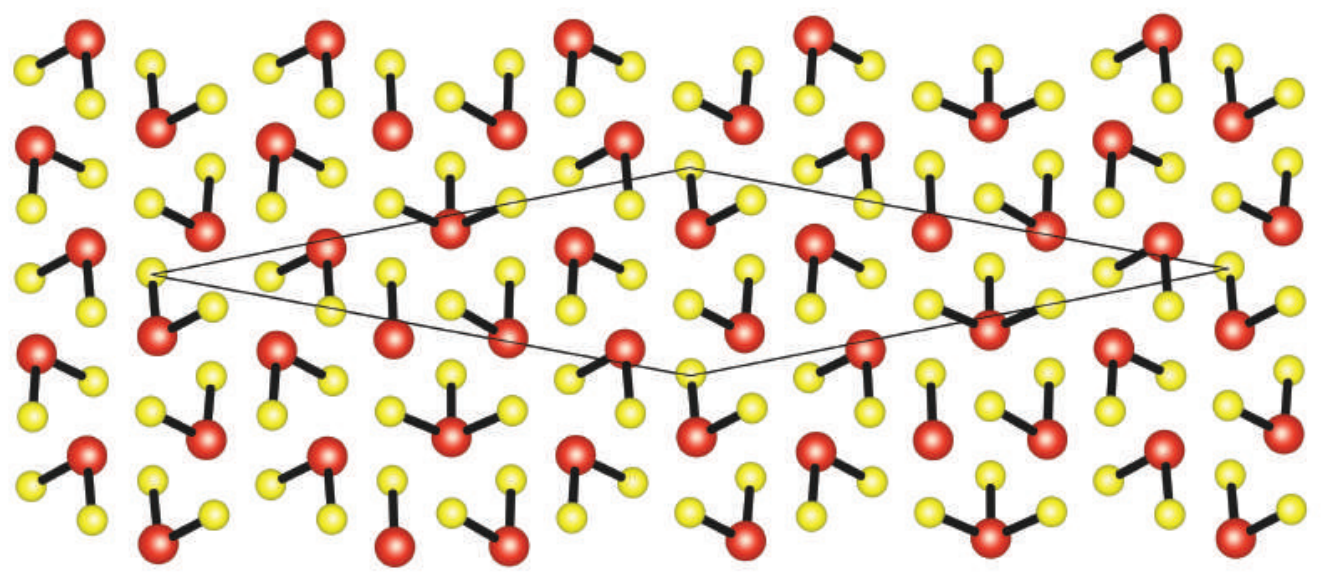

(b) TP $q=3 / 5 \mathrm{~K}$

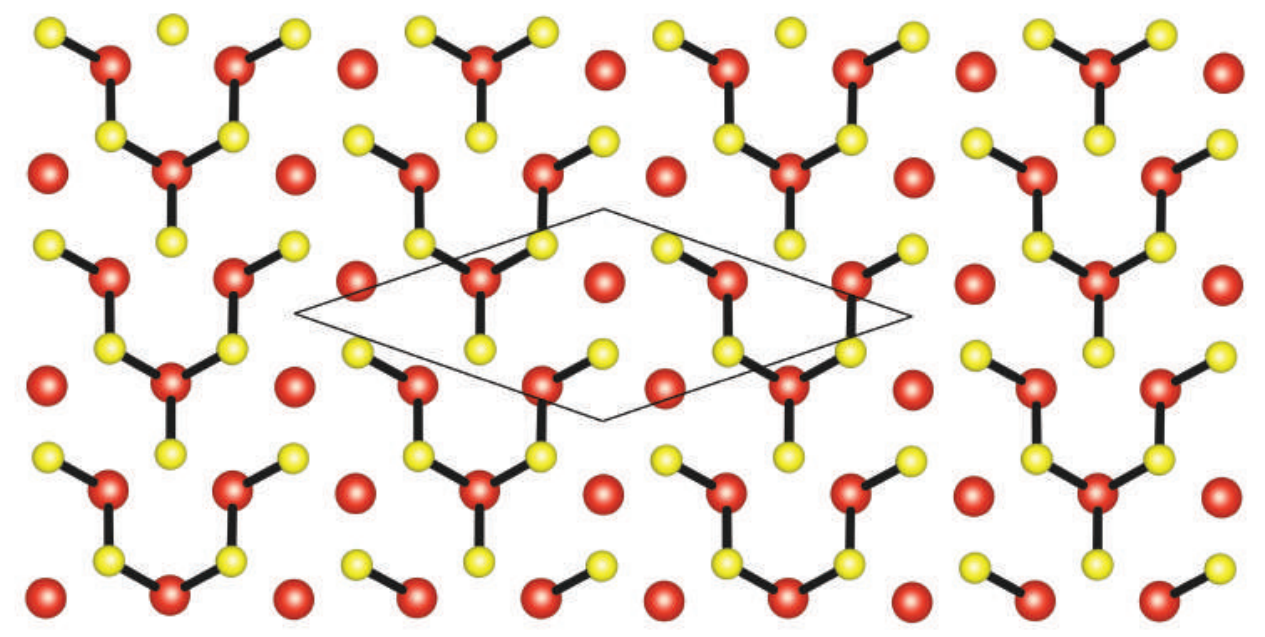

Figure 4.2: Orthographic projection along the out-of-plane axis of the (a) FM $U=0 q=2 / 3 \mathrm{~K}$ OCT and (b) FM $U=3.8 \mathrm{eV} q=3 / 5 K \mathrm{TP}$ relaxed structures. Vanadium (sulfur) ions are indicated by red (yellow) spheres and the thick black lines show the shortest $\mathrm{V}-\mathrm{S}$ bonds. The unit cell is indicated by thin black lines. 
FM structure, performing a further structural relaxation of the NSP $q=2 / 3 K$ OCT CDW structure with FM initialization leads to an additional small $(<1 \mathrm{meV}$ ) energy lowering (see Fig. 4.5). In this structure, depicted in Fig. 4.2(a), distinct V sites have one, two, or three nearest-neighbor S atoms instead of the six of the pristine OCT structure. The CDW has substantially suppressed the $\mathrm{V}$ magnetic moments to $0.0-0.2 \mu_{B}$, which is consistent with the weak correlations observed by Mulazzi et al. However, the $\mathrm{V}-\mathrm{S}$ and $\mathrm{V}-\mathrm{V}$ distances exhibit massive variations of 2.2-2.6 and 3.0-3.7 $\AA$, respectively. Sun et al. found that x-ray absorption fine spectroscopy (XAFS) data within the CDW phase was better interpreted by assuming two distinct $\mathrm{V}-\mathrm{V}$ distances (as opposed to one); a difference in $\mathrm{V}-\mathrm{V}$ distance of 0.19 $\AA$ was found [Sun et al. (2015)]. Therefore, DFT is severely overestimating the structural deformation in the CDW state and beyond-DFT approaches will be necessary to describe the OCT CDW phase; we address this point in detail using DFT $+U$ in Sec. 4.3.4. Also, additional experimental studies would be helpful to understand the lack of long-range CDW found using high-pressure synthesis.

\subsubsection{Non-spin-polarized DFT electronic structure}

The NSP band structure and density of states for TP $\mathrm{VS}_{2}$ are shown in Fig. 4.3. We do find an isolated low-energy band like in the crystal field picture shown in the top panel of Fig. 4.1, but there is a major difference with the simple schematic. The projected density of states shows this isolated band is mainly of $d$ character, while the unoccupied manifold above it has slightly less predominant $d$ character (i.e., stronger hybridization with $\mathrm{S} p$ ); the manifold below is predominantly $\mathrm{S} p$ with some hybridization with $\mathrm{V} d$. However, projecting

the $\mathrm{V} d$ density of states onto just the $A_{1}^{\prime}$ orbital $\left(d_{3 z^{2}-r^{2}}\right)$ reveals the main discrepancy with the simple schematic: the isolated band is only roughly half $A_{1}^{\prime}$ character and the remaining half is $E^{\prime}$ character. This puzzle was first noted by Kertesz and Hoffman in the context of TMDCs several decades ago [Kertesz and Hoffmann (1984)]. 


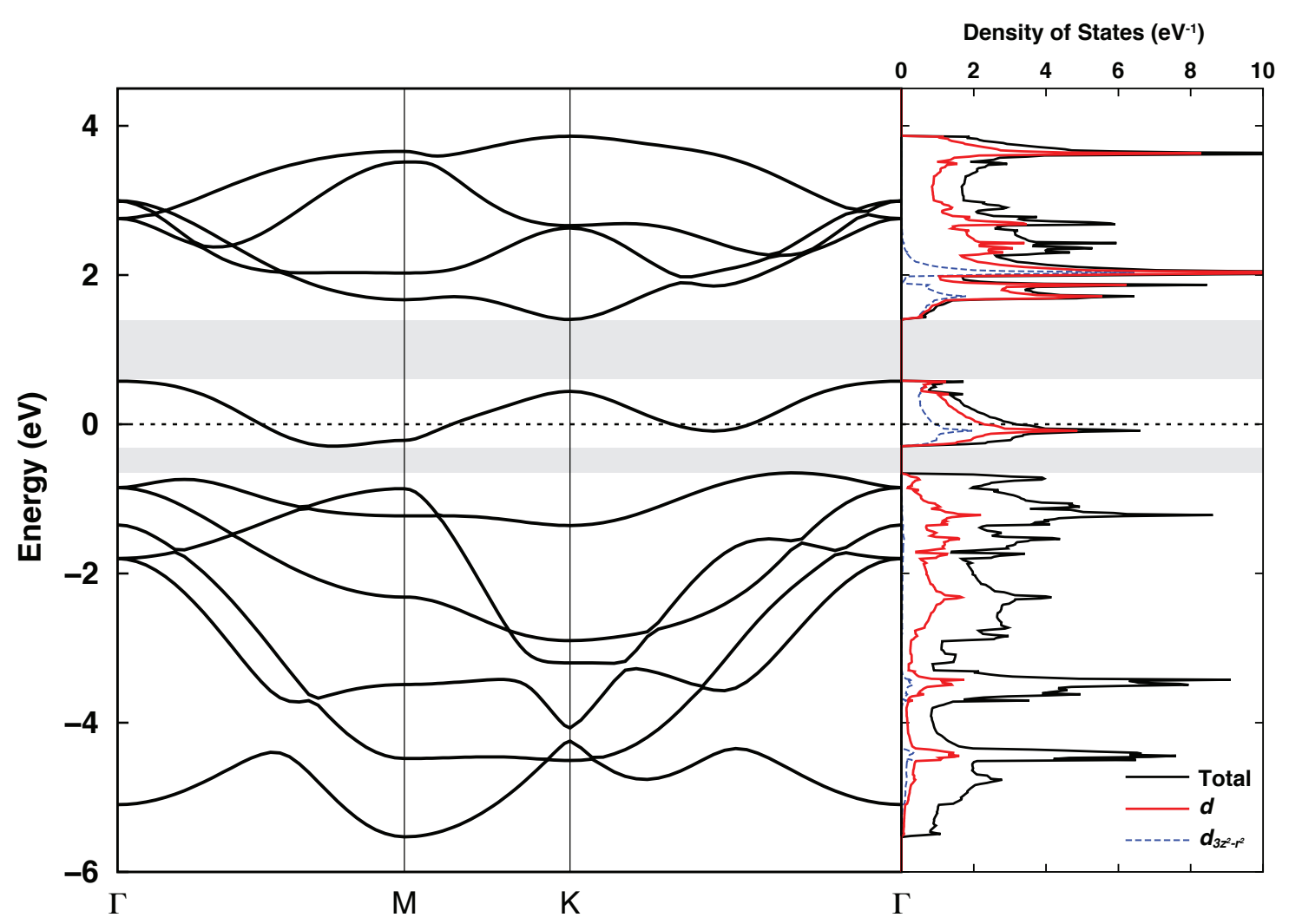

Figure 4.3: NSP electronic band structure and total (solid black line), $d$ (solid red line), and $d_{3 z^{2}-r^{2}}$ (dashed blue line) density of states for TP $\mathrm{VS}_{2}$ within DFT. The black dotted line indicates the Fermi energy and the shaded areas illustrate the gaps around the isolated low-energy band. The $k$-point labels $\Gamma, M$, and $K$ correspond to the center, edge midpoint, and corner of the Brillouin zone, respectively. 
(a)

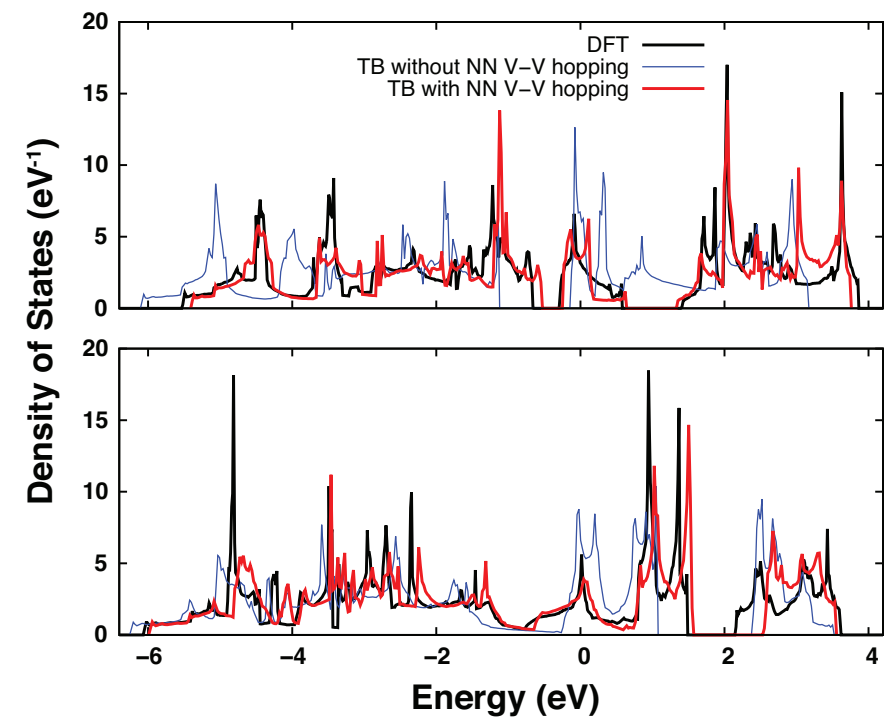

(b)
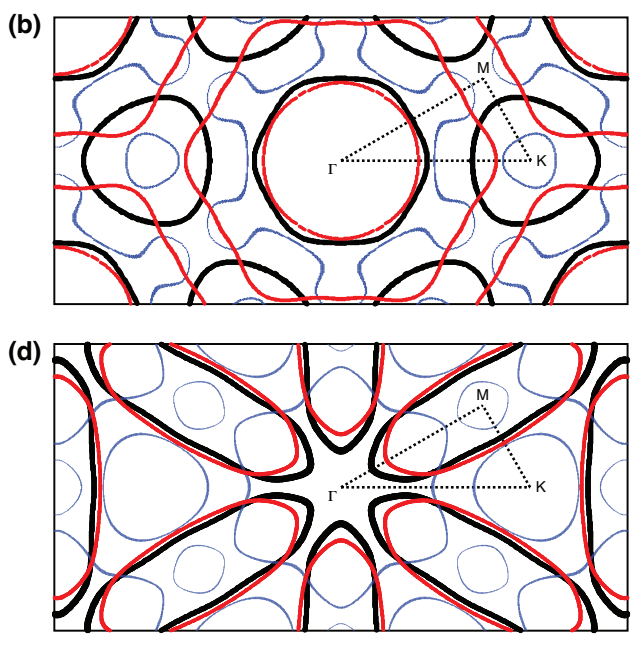

Figure 4.4: (a) Density of states and (b) Fermi surface for NSP TP $\mathrm{VS}_{2}$. The thick black lines correspond to DFT, while the thick red (thin blue) lines indicate tight binding results with (without) $\mathrm{NN} \mathrm{V-V}$ hopping matrix elements. The dotted lines show the irreducible Brillouin zone. Corresponding plots for OCT $\mathrm{VS}_{2}$ are shown in panels $(\mathrm{c})$ and $(\mathrm{d})$. 
In order to resolve this anomaly and to gain further insight into the electronic structure of the TP phase, we compute MLWFs for the full $p$ - $d$ manifold of $\mathrm{TP} \mathrm{VS}_{2}$, which results in atom-centered V $d$-like and S p-like orbitals. The Hamiltonian is represented in the MLWF basis, and we explore the impact of removing various matrix elements in the Hamiltonian corresponding to $\mathrm{V}-\mathrm{S}$ and $\mathrm{V}-\mathrm{V}$ hoppings; $\mathrm{S}-\mathrm{S}$ hoppings are always retained. A similar analysis is performed for the OCT phase for comparison.

Panels (a) and (c) of Fig. 4.4 show the density of states from the MLWF Hamiltonian for NSP TP and $\mathrm{OCT} \mathrm{VS}_{2}$ (black curves), respectively, which are identical to those of DFT by construction. The OCT structure, unlike the TP structure, does not have an isolated low-energy band since the crystal field splitting of the $T_{2 g}$ into $A_{1 g}$ and $E_{g}^{\prime}$ is relatively weak as is also typical for oxides in this structure. Now we examine the tight binding (TB) approximation in which we remove all $\mathrm{V}-\mathrm{S}$ and $\mathrm{V}-\mathrm{V}$ matrix elements beyond nearest neighbor (NN) (thick red lines). In both phases, we qualitatively reproduce all of the gaps and other prominent features of the spectra. For both structures, we find $\mathrm{V}-\mathrm{V}$ hopping beyond NN is negligible, and therefore all of the quantitative deviation between the black and the red curves is due to $\mathrm{V}-\mathrm{S}$ hopping beyond $\mathrm{NN}$.

If we only include $\mathrm{NN}$ - $-\mathrm{S}$ hoppings and no NN V-V hoppings (thin blue lines) we still capture the qualitative features of the spectra for the OCT structure, though there are now large quantitative differences. However, for TP phase there is a qualitative change: there is no longer a gap between the isolated $d$ band and the higher-energy $d$ bands. Therefore, the $\mathrm{V}-\mathrm{V}$ hopping plays a strong contribution in splitting off the isolated band. Furthermore, it addresses the observation presented by Kertesz and Hoffman. The fact that the NN V$\mathrm{V}$ hoppings have a strong interorbital component explains why $A_{1}^{\prime}$ only contributes half of character of the isolated band. Interestingly, we also find that the rapid decay of these direct TM-TM hoppings with strain explains the semiconductor-to-semimetal transition in the isostructural $d^{2}$ material $\mathrm{MoS}_{2}$ under strain [Scalise et al. (2012)].

Panels (b) and (d) of Fig. 4.4 illustrate the Fermi surfaces of the TP phase and OCT 
phase, respectively. In DFT, the Fermi surface of the TP structure has hole pockets centered at $\Gamma$ and $K$, while that of the OCT structure has a single cigar-shaped electron pocket centered at $M$. For the OCT structure the TB approximation is sufficient to properly capture the Fermi surface topology, but for the TP structure this is not the case and longer-range $\mathrm{V}-\mathrm{S}$ hopping is needed.

At this level of theory we predict an isolated low-energy band in the TP phase, but as discussed in the next section there is a ferromagnetic instability once spin polarization is included even at the DFT level. This strongly suggests electronic correlations will be important in the TP phase of this material, which therefore is our focus for the remainder of this paper.

\subsubsection{DFT energy level diagram}

The total energy of different structures and magnetic configurations of monolayer $\mathrm{VS}_{2}$ within DFT is shown in Fig. 4.5. For the NSP states, the TP structure is lower in energy than the OCT structure by $15 \mathrm{meV}$. For both structures, the formation of a FM state results in a significant energy lowering compared to the NSP state. The magnitude of the energy decrease is $13 \mathrm{meV}$ for $\mathrm{OCT}$ and $49 \mathrm{meV}$ for TP. In the FM state, $\mathrm{V}$ in the TP structure is fully spin polarized with a magnetic moment of $1.0 \mu_{B}$ whereas for the OCT structure the moment is only $0.5 \mu_{B}$, indicating that the TP phase exhibits stronger signatures of electronic correlations. For the OCT phase one must also consider the CDW phase, which lowers the OCT energy by $12 \mathrm{meV}$ compared to the FM state and greatly weakens the magnetism giving moments of only $0.0-0.2 \mu_{B}$. Ultimately, the TP FM state is the ground state since it is still far lower in energy (38 meV) than the OCT FM CDW phase. The only remaining task is to provide evidence that there are no other magnetic or phonon instabilities.

To confirm the exchange is $\mathrm{FM}$ in $\mathrm{VS}_{2}$, we also investigate $q=M$ and $q=3 / 4 \mathrm{~K}$ antiferromagnetic (AFM) configurations. For the TP phase, only the striped $(q=M)$ AFM 


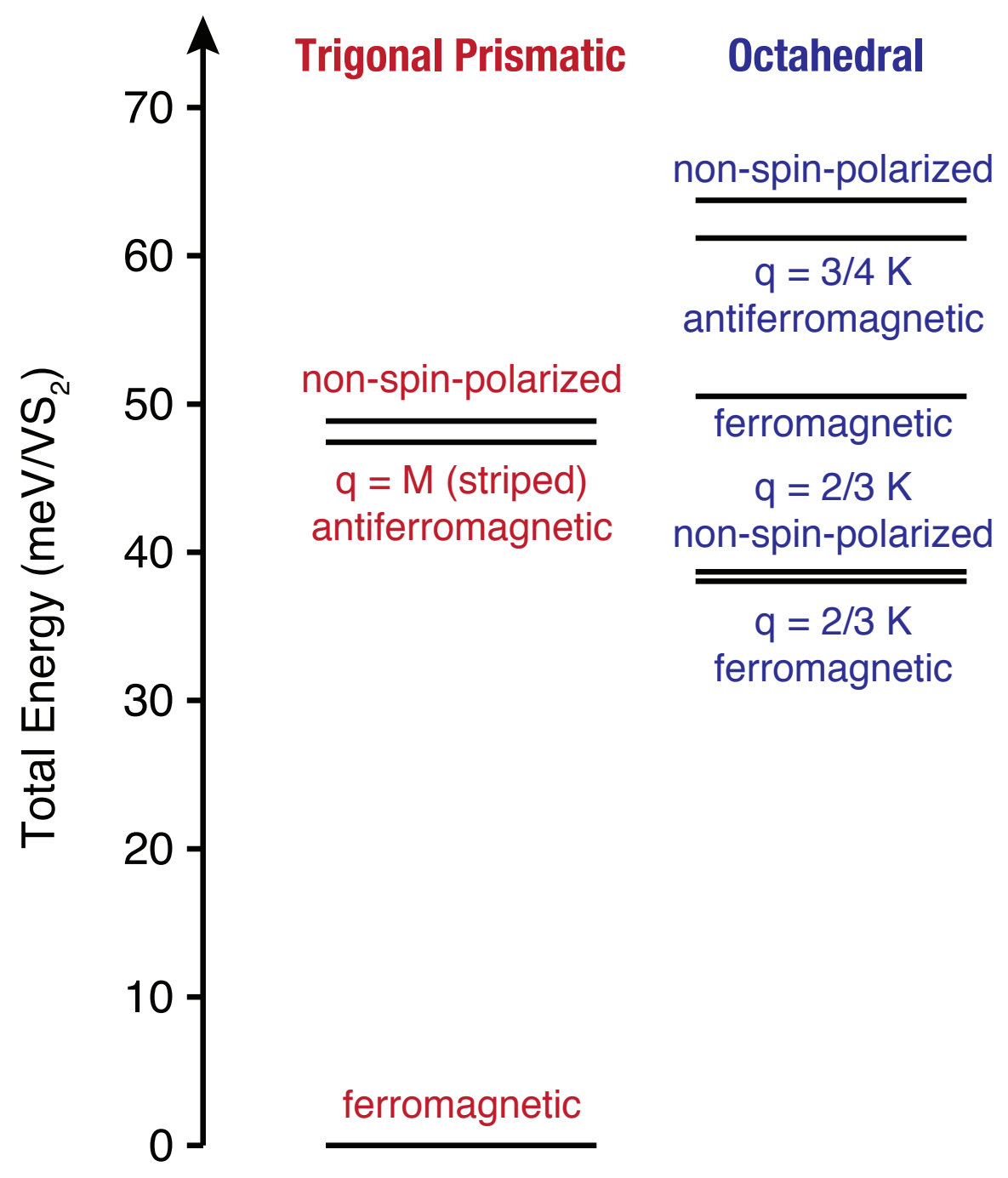

Figure 4.5: Energy level diagram for TP (left, in red) and OCT (right, in blue) $\mathrm{VS}_{2}$ within DFT. The energy of the FM TP state is used as a reference energy. 
configuration is found to converge. This metastable state is metallic with small V magnetic moments of $\pm 0.2 \mu_{B}$ and is only $1.4 \mathrm{meV}$ lower in energy than the NSP state. Therefore, $\mathrm{TP} \mathrm{VS}_{2}$ strongly prefers ferromagnetism and we interpret it as a "Stoner insulator" rather than a Mott insulator at the level of spin-dependent DFT, given that a gap does not persist for an arbitrary magnetic ordering. For the OCT structure a metastable $q=3 / 4 \mathrm{~K} \mathrm{AFM}$ configuration is found only $2.4 \mathrm{meV}$ lower in energy than the NSP state, and it similarly is metallic with small V moments of $\pm 0.4 \mu_{B}$. The FM nature of the exchange in this system is not unexpected since the $\mathrm{V}-\mathrm{S}-\mathrm{V}$ angle is 84-85 degrees, close to the 90-degree ferromagnetism given by the Goodenough-Kanamori rules [Goodenough (1955, 1958); Kanamori (1959)].

We compute the phonon dispersion and density of states of FM TP VS 2 , shown in Fig. 4.6, to assess the dynamic stability of this phase. The out-of-plane acoustic (ZA) branch has the $\omega \sim q^{2}$ form near $\Gamma$ characteristic of two-dimensional materials. There is no frequency gap between the acoustic and optical branches. The out-of-plane optical (zO) branches are the highest-frequency phonons. Since there are no modes with imaginary frequency, this phase is stable at the level of DFT.

The above analysis of the magnetism and the phonons allows us to conclude that the FM TP phase is the ground state within DFT. One would not interpret this as a Mott insulator within DFT given that the band gap does not persist for all spin configurations.

\subsubsection{Impact of on-site Hubbard $U$}

We use the linear response approach [Cococcioni and de Gironcoli (2005)] to estimate the correlation strength $U$ for $\mathrm{V}$ in $\mathrm{VS}_{2}$. Computing screened interactions for use in beyond-DFT

methods is still an active area of research, but the linear response approach is useful to set a baseline for the expected value of $U$. For FM states, we obtain $U=3.84 \mathrm{eV}$ for the TP phase and $U=3.99 \mathrm{eV}$ for the OCT phase. For the TP phase, we also compute the $U$ for the NSP state and obtain $4.14 \mathrm{eV}$. These values are generally smaller than those of oxides of vanadium 


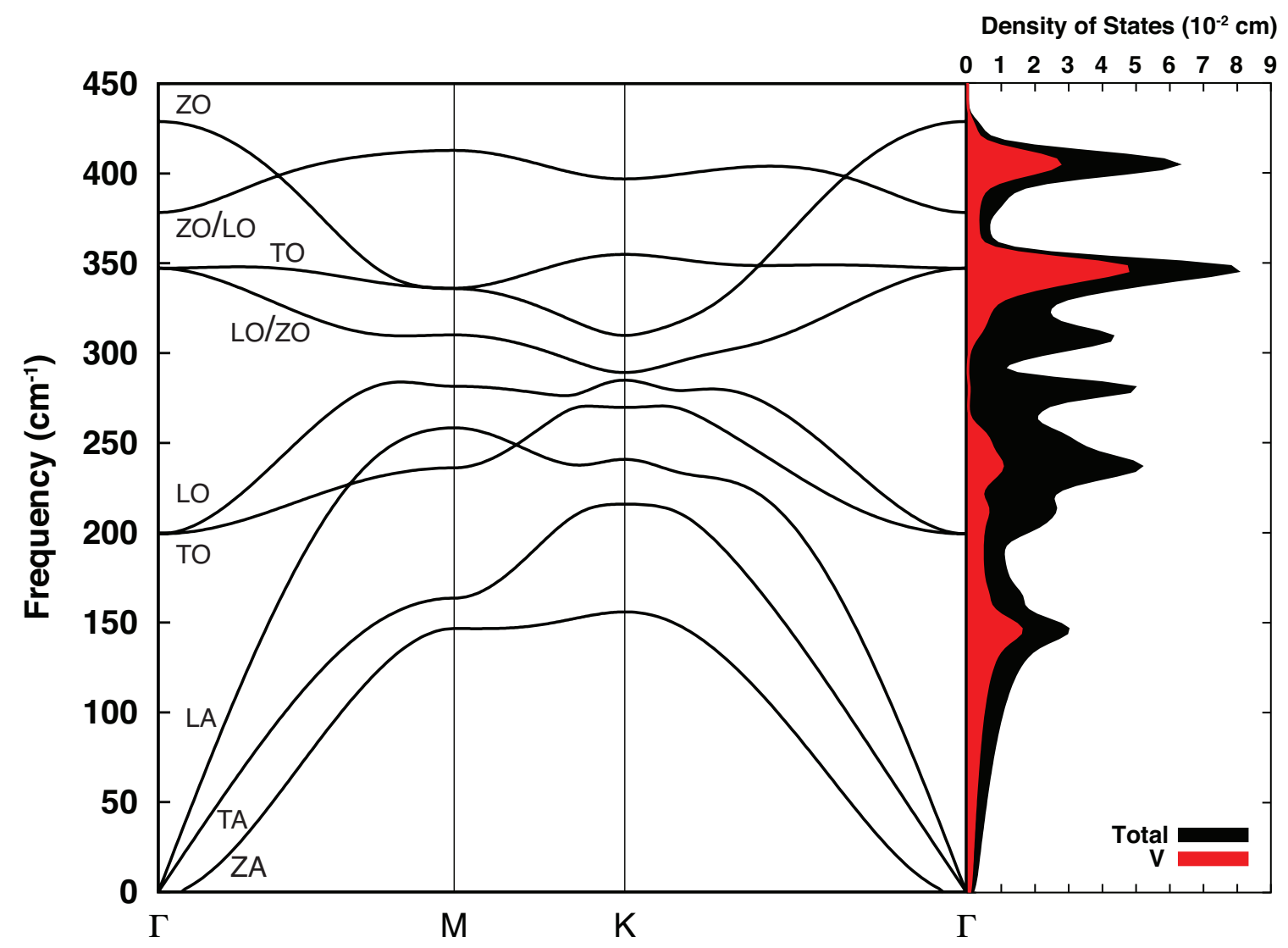

Figure 4.6: Phonon dispersion relation and total (black) and V-projected (red) phonon density of states for FM TP $\mathrm{VS}_{2}$ within DFT. The band labels identify the mode character near the $\Gamma$ point. $\mathrm{z}, \mathrm{T}, \mathrm{L}, \mathrm{A}$ and $\mathrm{O}$ refer to out-of-plane, transverse, longitudinal, acoustic, and optical branches, respectively. 
[Xu et al. (2015)] and larger than those of sulfides of titanium and tantalum [Sánchez et al. (2008); Darancet et al. (2014)]. Ultimately, one still needs to carefully investigate the effect of $U$ on the physical observables given the methodological uncertainties.

\begin{tabular}{lcccc}
\hline \hline & $U(\mathrm{eV})$ & $\Delta \mathrm{E}(\mathrm{meV})$ & $\mathrm{V}-\mathrm{S}$ bond length range $(\AA)$ & V mag. mom. range \\
\hline NSP $q=3 / 5 K$ & 0 & -17 & $2.22-2.52$ & - \\
& 1 & -20 & $2.23-2.51$ & - \\
& 2 & -33 & $2.24-2.51$ & - \\
& 3 & -60 & $2.25-2.51$ & - \\
\hline NSP $q=2 / 3 K$ & 0 & -25 & $2.18-2.57$ & - \\
& 1 & -27 & $2.20-2.56$ & - \\
& 2 & -34 & $2.21-2.55$ & - \\
\hline FM $q=3 / 5 K$ & 3 & -60 & $2.25-2.52$ & $0.03-0.38$ \\
& 1 & -7 & $2.21-2.53$ & $1.17-1.19$ \\
& 2 & -2 & $2.30-2.42$ & $1.21-1.39$ \\
& 3 & -14 & $2.26-2.51$ & $1.30-1.40$ \\
\hline FM $q=2 / 3 K$ & 0 & -1 & $2.37-2.42$ & $-0.02-0.18$ \\
& 1 & -12 & $2.18-2.57$ & $1.14-1.20$ \\
& 2 & -10 & $2.26-2.47$ & $1.27-1.32$ \\
& 3 & -1 & $2.27-2.49$ & $1.28-1.33$ \\
\hline \hline
\end{tabular}

Table 4.1: Total energy change per formula unit with respect to the pristine structure of the same magnetic state, V-S bond length range, and $\mathrm{V}$ magnetic moment range for the NSP and FM states of OCT $\mathrm{VS}_{2}$ with $q=3 / 5 K$ and $q=2 / 3 K$ relaxed structures.

Another useful benchmark that could provide a bound for $U$ is the CDW in the OCT phase. We performed structural relaxations to check if the CDW is still captured for finite $U$. The total energy lowering $\Delta \mathrm{E}, \mathrm{V}-\mathrm{S}$ bond length range, and $\mathrm{V}$ magnetic moment range for the relaxed structures are given in Table 4.1 for $\mathrm{NSP}$ and $\mathrm{FM} \mathrm{OCT} \mathrm{VS}_{2}$ for $q=3 / 5 \mathrm{~K}$ and $q=2 / 3 K$. For the NSP states the energy lowering from the CDW increases substantially with $U$ and is $60 \mathrm{meV}$ for $U=3 \mathrm{eV}$. For the FM states, the CDW persists for moderate values of $U$ but it is substantially dampened once $U$ is $3 \mathrm{eV}$ with a total energy lowering of only $1 \mathrm{meV}$. However, at $U=3$ we find evidence for a new $q=2 / 3 \mathrm{~K}$ CDW ground state with AFM-like correlations. This system is a ferrimagnetic metal with $2 \mathrm{~V}$ moments of 1.3 
$\mu_{B}, 3 \mathrm{~V}$ moments of $1.4 \mu_{B}$, and $4 \mathrm{~V}$ moments of $-1.2 \mu_{B}$. We refer to it as an AFM state for simplicity since the total magnetization is only $0.21 \mu_{B}$ per formula unit.

Further evidence for this tendency for AFM correlations in $\mathrm{OCT} \mathrm{VS}_{2}$ for larger $U$ comes from calculations of the $q=M$ and $q=3 / 4 K$ AFM states. For $U=3 \mathrm{eV}$ the $q=M$ and $q=3 / 4 K$ AFM states are also lower in energy than the pristine FM state by 29 and $19 \mathrm{meV}$, respectively. The $q=2 / 3 \mathrm{~K}$ AFM CDW state is even lower in energy, $39 \mathrm{meV}$ lower than the pristine FM state, and therefore is the ground state. For $U=4 \mathrm{eV}$ this trend persists as $q=M$ and $q=3 / 4 K$ phases with anti-aligned magnetic moments are lower in energy than the pristine FM phase by 35 and $29 \mathrm{meV}$, respectively. It should be emphasized that these anti-aligned magnetic states are strongly coupled to the structural distortions; performing an unrelaxed $U=3 \mathrm{eV}$ calculation based on the FM $U=0$ or $U=3 \mathrm{eV}$ relaxed structure of the primitive unit cell (i.e., without any CDW) demonstrates that the FM spin ordering persists as the ground state.

To assess which regime of $U$ best agrees with experiments on the CDW phase, we compare the $\mathrm{V}-\mathrm{V}$ and $\mathrm{V}-\mathrm{S}$ distances of our calculated structures to those of known experiments in Fig. 4.7. For the $\mathrm{V}-\mathrm{V}$ distance the high-temperature value of Sun et al. agrees well with that of Murphy et al., which may be reasonable since the temperature is approaching the CDW transition at $305 \mathrm{~K}$. Gauzzi et al., who do not find a long-range CDW, observe a slightly larger $\mathrm{V}-\mathrm{V}$ distance at low temperature. The work of Sun et al. is the only work that presents atomic distances at low temperature well within the CDW phase; they report a $\mathrm{V}-\mathrm{V}$ distance difference of $0.19 \AA$.

Applying $\mathrm{DFT}+U$ while not allowing spontaneously broken translational symmetry, the $\mathrm{V}-\mathrm{V}$ and $\mathrm{V}-\mathrm{S}$ distances of the pristine FM state increase roughly linearly with $U$. For this state, within DFT $(U=0)$ PBE predicts larger bond lengths than the local density approximation (LDA) as is typical. As discussed in Sec. 4.3.1, for $U=0$ the range of $\mathrm{V}-\mathrm{V}$ distances of the $q=2 / 3 \mathrm{~K} \mathrm{FM} \mathrm{CDW} \mathrm{phase}(0.70 \AA)$ is over 3.5 times the low-temperature XAFS measurement from Sun et al. For $U=1$ and $2 \mathrm{eV}$ the range we compute is smaller 


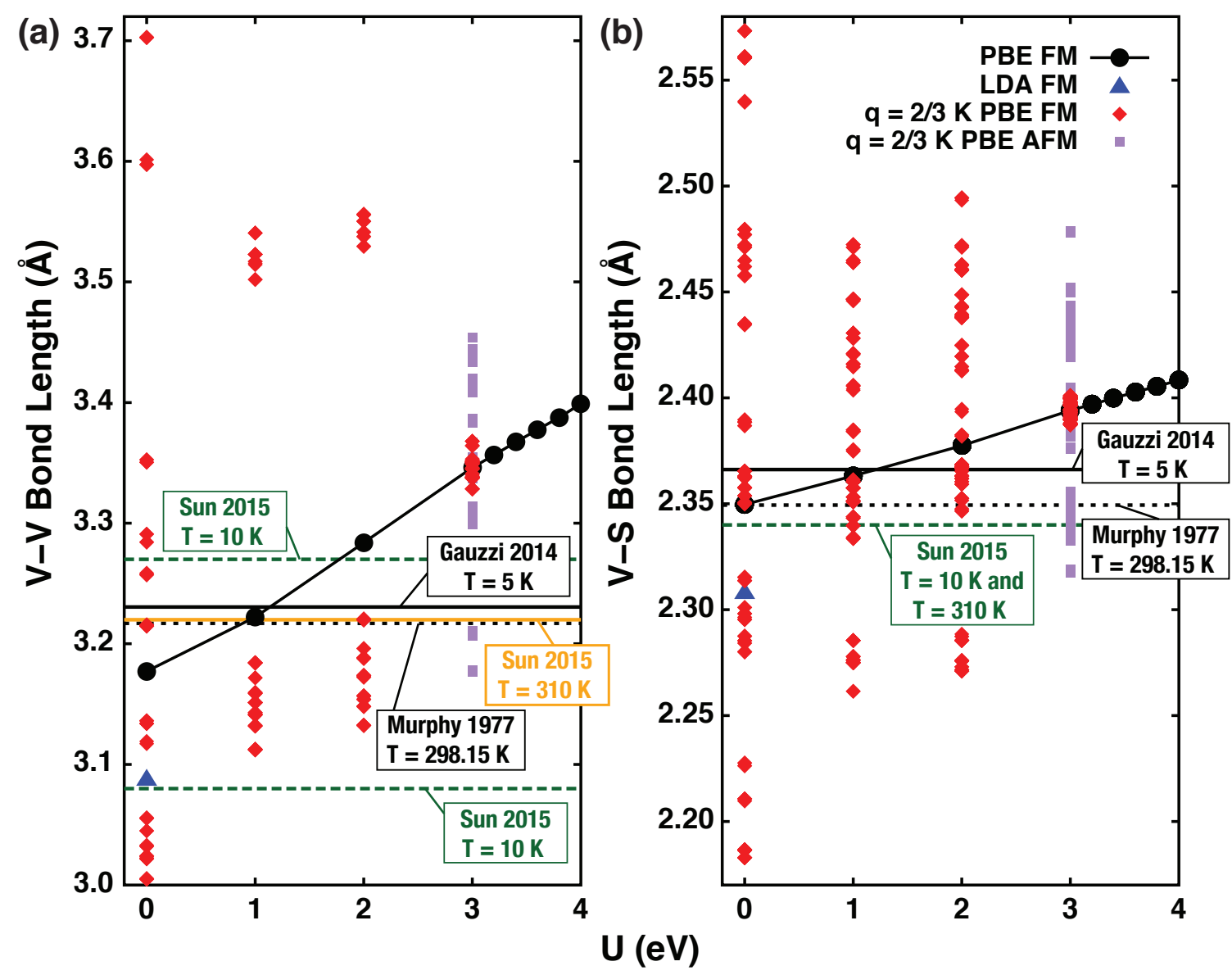

Figure 4.7: (a) $\mathrm{V}-\mathrm{V}$ and (b) $\mathrm{V}-\mathrm{S}$ distances for $\mathrm{OCT} \mathrm{VS}_{2}$ in the pristine $\mathrm{FM}$ phase, $q=2 / 3 K$ FM CDW phase, and $q=2 / 3 K$ AFM CDW phase as a function of $U$. The two green dashed lines for the low-temperature experiment of Sun et al. in panel (a) correspond to the two measured $\mathrm{V}-\mathrm{V}$ distances. For comparison, the $U=0$ value for the pristine FM phase is also shown within the local density approximation (LDA). 
but still over twice the experimental value, while the range collapses to only $0.04 \AA$ for $U=3 \mathrm{eV}$. Alternatively, reasonable agreement with experiment occurs for the $U=3 \mathrm{eV}$ $q=2 / 3 K$ AFM CDW phase. This phase still contains an appreciable CDW distortion, unlike the corresponding FM phase, and the range of $\mathrm{V}-\mathrm{V}$ distances of $0.28 \AA$ is comparable to that in experiment. Furthermore, the metallic nature of this phase (unlike the gapped FM CDW phase) is qualitatively consistent with the experimental resistivity [Murphy et al. (1977); Mulazzi et al. (2010); Sun et al. (2015)]. Therefore, an appreciable $U$ value of around

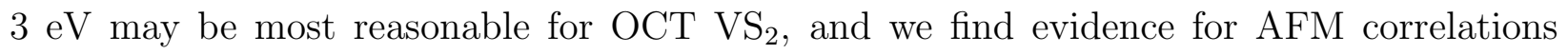
in this regime. The $\mathrm{V}-\mathrm{S}$ bond lengths show a similar trend: the $q=2 / 3 K \mathrm{FM}$ CDW phase exhibits a massive range of values for $U=0$ that is dampened for $U=1$ and 2 $\mathrm{eV}$ and nearly disappears for $U=3 \mathrm{eV}$. We note that Sun et al. reports only a single temperature-independent V-S bond length, however. A detailed structural refinement from experiment would be instrumental for a more stringent evaluation of available first-principles methodologies.

$\mathrm{DFT}+U$ corresponds to a Hartree-Fock (mean-field) solution to the quantum impurity problem of dynamical mean-field theory [Georges et al. (1996); Kotliar et al. (2006)]. Given the manner in which Hartree-Fock tends to overemphasize the effects of interactions, it would not be surprising to require a smaller value of $U$ relative to that of linear response to provide a proper description. Especially given that there are currently no experiments for the TP phase, the above analysis indicates the need to explore a range of $U$ values in what follows.

We explore the effect of $U$ on the electronic spectrum of FM TP $\mathrm{VS}_{2}$ using DFT $+U$. As shown in Fig. 4.8(a), for $U=0$ already there is a small band gap of $30 \mathrm{meV}$ generated by the exchange splitting of the $A_{1}^{\prime}$ state. With increasing $U$ the spin-down $A_{1}^{\prime}$ state is shifted up in energy, which increases the band gap up to $0.6 \mathrm{eV}$; the band gap saturates once the spin-up $E^{\prime}$ levels become the lowest unoccupied states. This value is somewhat smaller than the $1.1 \mathrm{eV}$ band gap obtained via hybrid functional calculations, which is presumably due to the nonlocality of the potential in the hybrid functional [Huang et al.]. For small $U$, the $U$ - 
4. Electronic correlations in monolayer $\mathrm{VS}_{2}$

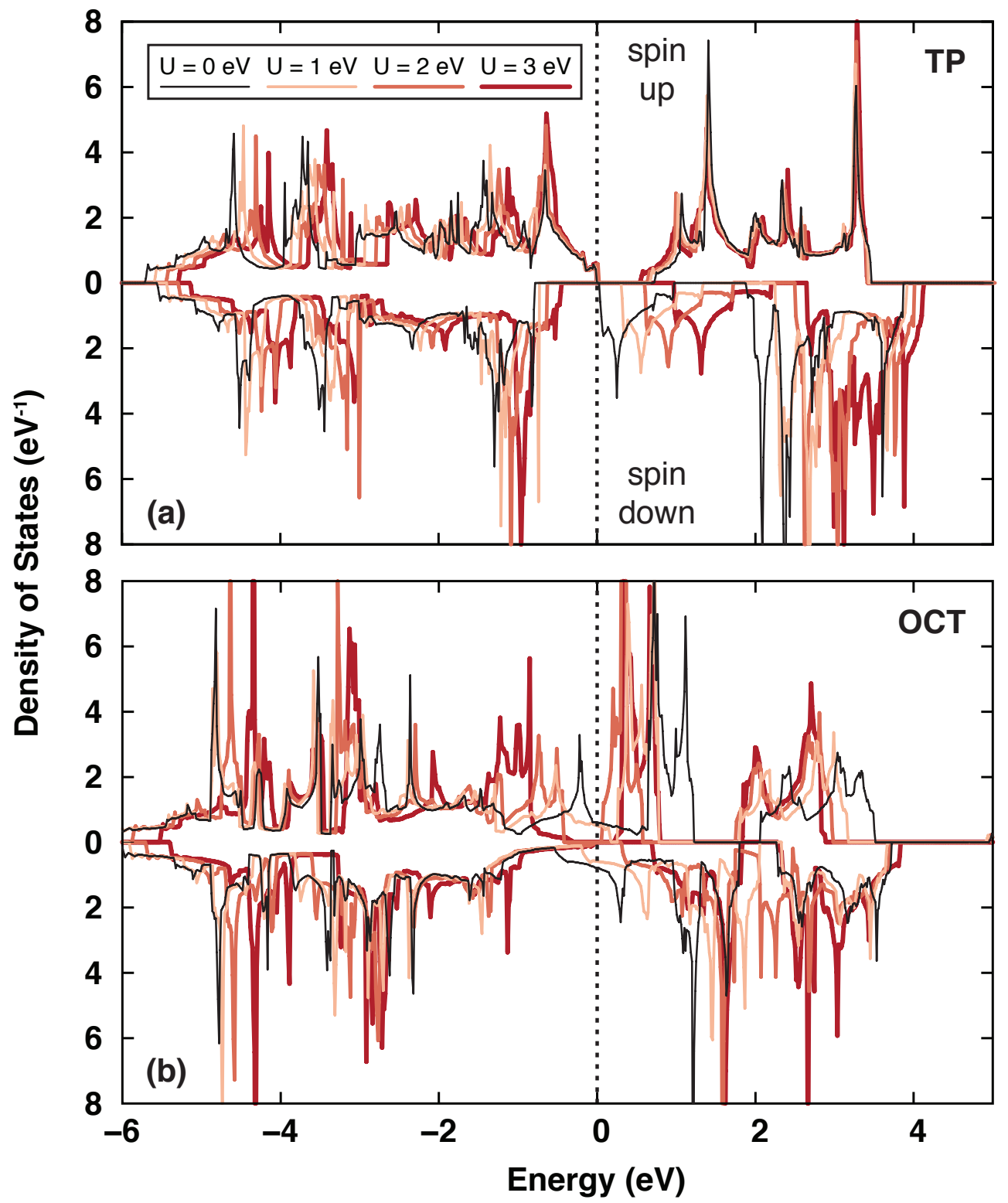

Figure 4.8: Electronic density of states for $\mathrm{FM} \mathrm{VS}_{2}$ in the (a) TP and (b) OCT phases for different values of $U$. The dotted black line indicates the Fermi level. 
induced energy shift of correlated orbital $\left|d_{\alpha}\right\rangle$ with occupancy $n_{\alpha}$ takes the form $U\left(1 / 2-n_{\alpha}\right)$ within $\mathrm{DFT}+U$, so one expects an occupied state $\left(n_{\alpha}=1\right)$ to shift down in energy by $U / 2$ and an unoccupied state $\left(n_{\alpha}=0\right)$ to shift up in energy by $U / 2$. In this case, however, the spin-up $d$ levels are significantly hybridized such that their occupancies are very close to 1/2 (i.e., 0.45-0.48) within DFT. This necessitates that the spin-up $d$ manifold is essentially fixed in energy for small $U$. The trend happens to persist over the full range of $U$ shown, which is responsible for the band gap saturation observed here as well as in a previous study [Huang et al.]. For comparison, the impact of $U$ on the density of states of FM OCT $\mathrm{VS}_{2}$ is shown in Fig. 4.8(b).

For $U$ of 2 and $4 \mathrm{eV}$ the metastable striped $q=M$ AFM configuration is 115 and 66 meV higher in energy than the FM state with a band gap of 0.1 and $0.7 \mathrm{eV}$ and $\mathrm{V}$ magnetic moments of \pm 0.6 and $\pm 1.3 \mu_{B}$, respectively. The insulating behavior for this higher-energy magnetic configuration indicates that the system has been driven into a regime of Mott physics, as crudely interpreted from $\mathrm{DFT}+U$; this is in contrast to the DFT description in terms of a Stoner instability.

We also examine the impact of $U$ on the phonon dispersion relation of the FM TP state to assess the dynamical stability of $\mathrm{VS}_{2}$. Figure 4.9 illustrates the main result. For $U=3.0$ $\mathrm{eV}$ the phonons are all still stable, as in the DFT case. For $U=3.2 \mathrm{eV}$ one can observe the formation of a small dip in the TA branch between $\Gamma$ and $K$. Once $U$ is equal to $3.4 \mathrm{eV}$, a soft mode is formed. There is an additional soft mode at $q=K$ whose eigenvalue is smaller in magnitude.

To corroborate and refine our finding of $U$-induced soft modes in the TP phase, we performed frozen phonon calculations at several $q$-points. The frozen phonon method removes the possibility of image interactions, which can cause errors in the supercell approach. For $U=3.4 \mathrm{eV}$ we find a $130 i \mathrm{~cm}^{-1}$ soft mode at the $K$ point, a $100 i \mathrm{~cm}^{-1}$ soft mode at $q=1 / 2 K$, and a $188 i \mathrm{~cm}^{-1}$ soft mode at $q=3 / 5 K$; this reveals that the supercell approach is qualitatively correct but with substantial quantitative errors. 
4. Electronic correlations in monolayer $\mathrm{VS}_{2}$

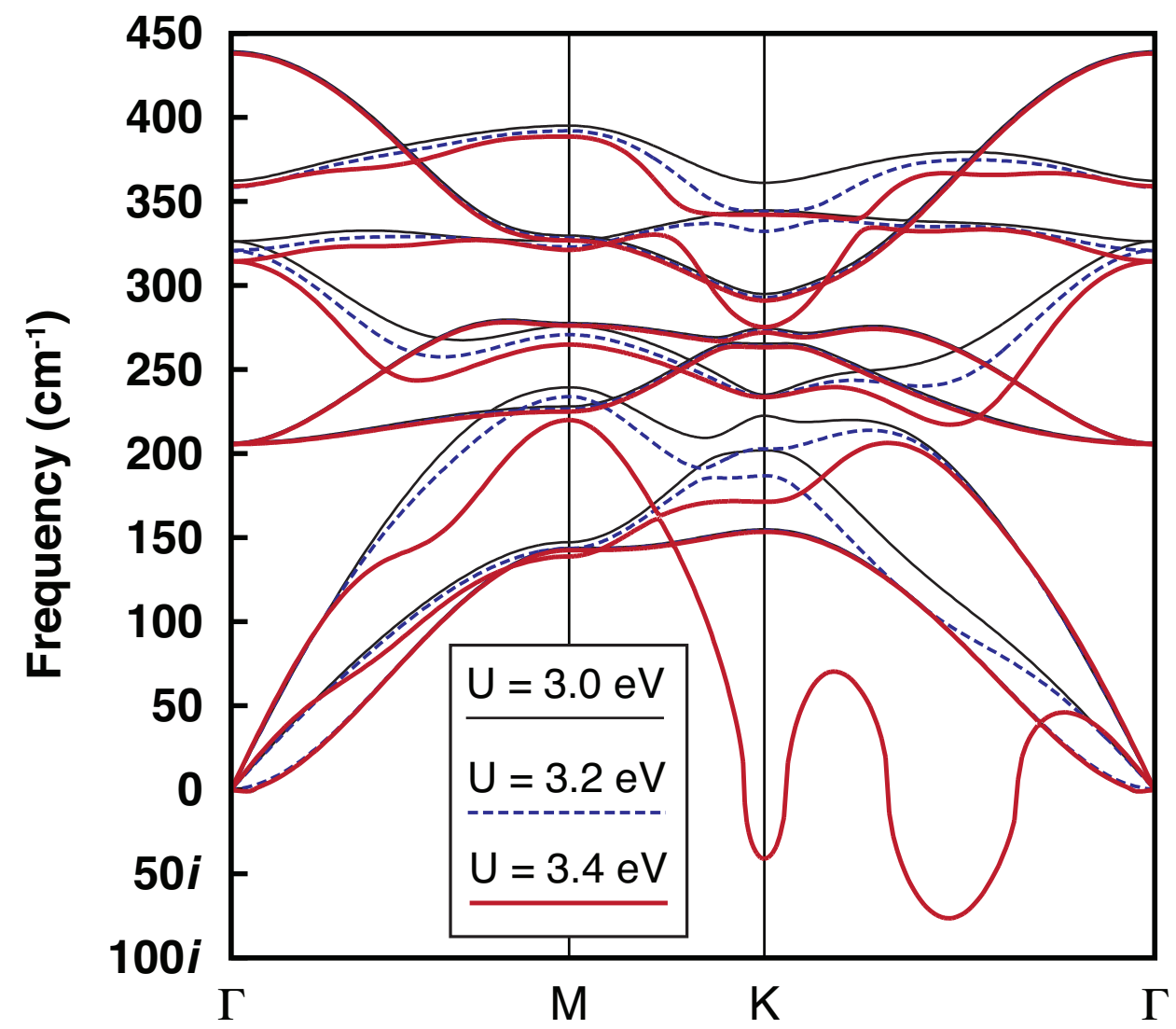

Figure 4.9: Phonon dispersion relation for FM TP $\mathrm{VS}_{2}$ for $U=3.0 \mathrm{eV}$ (thin solid black lines), $U=3.2 \mathrm{eV}$ (dashed thin blue lines), and $U=3.4 \mathrm{eV}$ (thick solid red line). 


\begin{tabular}{ccccc}
\hline \hline & $U(\mathrm{eV})$ & $\Delta \mathrm{E}(\mathrm{meV})$ & $\mathrm{V}-\mathrm{S}$ bond length range $(\AA)$ & V magnetic moment range \\
\hline \multirow{6}{*}{$q=K$}
\end{tabular}$\left(\mu_{B}\right)$

Table 4.2: Total energy change per formula unit, $\mathrm{V}-\mathrm{S}$ bond length range, and $\mathrm{V}$ magnetic moment range for FM TP $q=K$ and $q=3 / 5 K$ relaxed structures for several $U$ values.

We performed structural relaxations for the two wavevectors with the softest phonon modes, $q=K$ and $q=3 / 5 K$, using supercells commensurate with those wavevectors. The total energy lowering $\Delta \mathrm{E}, \mathrm{V}-\mathrm{S}$ bond length range, and $\mathrm{V}$ magnetic moment range for the relaxed structures are given in Table 4.2. For $U=3.2 \mathrm{eV}$ no structural distortion is found for either wavevector. With larger $U$ values, the relaxed structures exhibit lower total energy and modulation of $\mathrm{V}-\mathrm{S}$ bond lengths and $\mathrm{V}$ magnetic moments. For $q=$ $3 / 5 K$ the magnitude of $\Delta \mathrm{E}$ increases monotonically from $1 \mathrm{meV}$ to $45 \mathrm{meV}$ as $U$ increases, corresponding to an enhanced CDW. The V-S bond lengths vary by as much as $0.09 \AA$ and the $\mathrm{V}$ magnetic moments differ by as much as $0.8 \mu_{B}$ at a given $U$. For $3.4 \mathrm{eV} \leq U \leq 3.8$ eV the $q=K$ soft mode also shows an appreciable but smaller energy lowering $(|\Delta E| \leq 10$ meV) with significantly smaller magnitudes of the differences in V-S bond length $(0.03 \AA)$ and V magnetic moment $\left(0.01 \mu_{B}\right)$; for $U>3.8 \mathrm{eV}$ this CDW state becomes higher in energy than the undistorted FM state. For $U=5 \mathrm{eV}$ we do not find a stable (or even metastable) $q=3 / 5 K$ or $q=K \mathrm{CDW}$ state, indicating the prediction of a CDW state for $\mathrm{TP} \mathrm{VS}_{2}$ only exists within a narrow window of $U$ values.

For $U \geq 4 \mathrm{eV}$, both the $q=3 / 5 K$ and $q=K$ soft modes disappear (not pictured). Frozen phonon calculations indicate that the smallest phonon frequency at $U=4 \mathrm{eV}$ is 126 

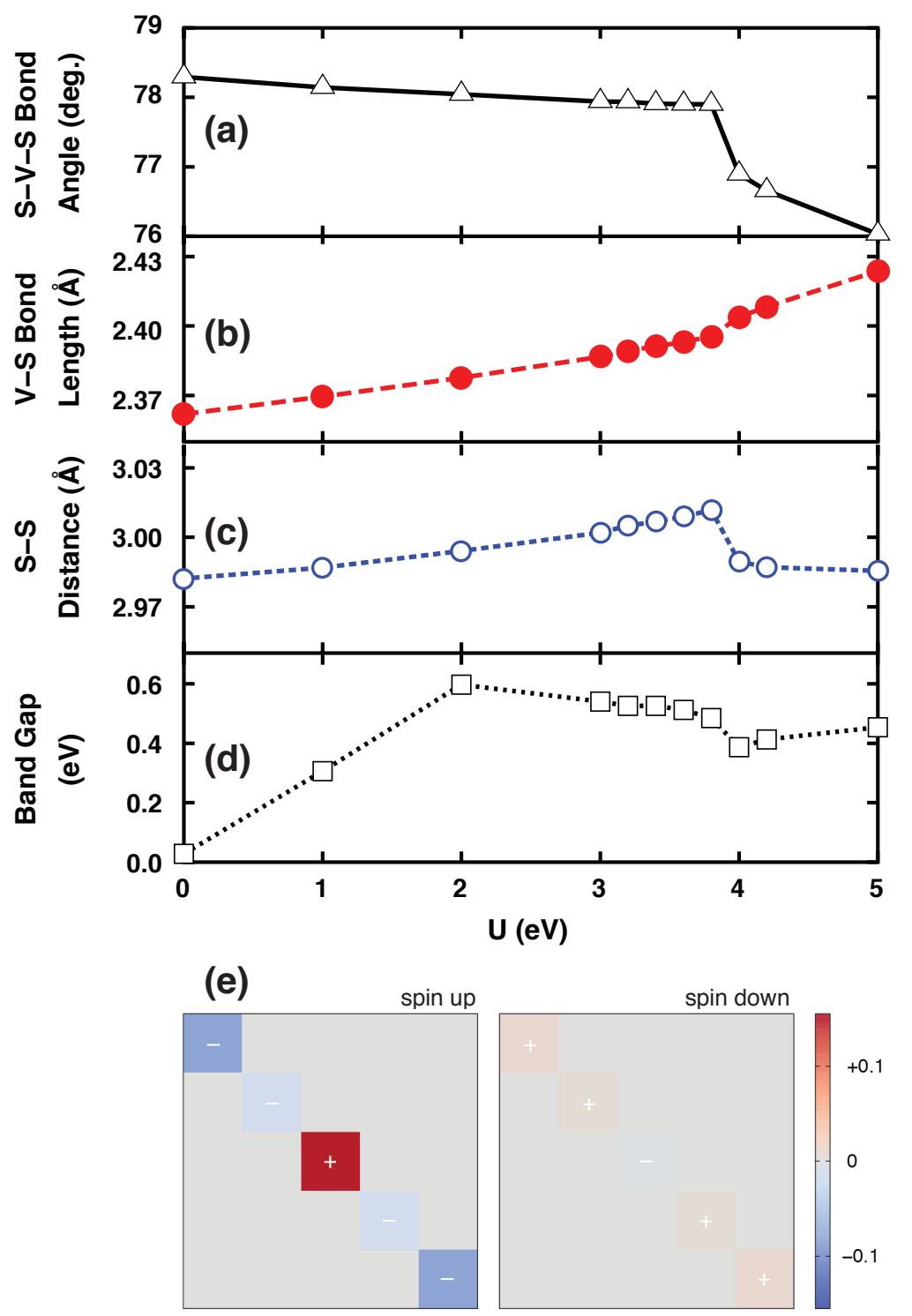

Figure 4.10: (a) S-V-S bond angle, (b) V-S bond-length, (c) out-of-plane S-S distance, and (d) electronic band gap as a function of $U$ for FM TP $\mathrm{VS}_{2}$. The density matrix difference for $U=4 \mathrm{eV}$ (ground state minus metastable state) for spin-up (left) and spin-down (right) electrons is displayed in panel (e). The matrix rows (columns) correspond to $d_{x y}, d_{y z} d_{3 z^{2}-r^{2}}, d_{x z}$, and $d_{x^{2}-y^{2}}$ states from top to bottom (left to right). 
$\mathrm{cm}^{-1}$ for $q=K, 97 \mathrm{~cm}^{-1}$ for $q=3 / 5 K$, and $79 \mathrm{~cm}^{-1}$ for $q=1 / 2 K$. In this regime of $4 \mathrm{eV}$ $\leq U<5 \mathrm{eV}$ we find that the $q=3 / 5 \mathrm{~K} \mathrm{CDW}$ phase is a separate lower-energy state that exists in addition to the metastable undistorted FM state.

The disappearance of the soft modes at $U \geq 4 \mathrm{eV}$ appears to be related to a separate electronic and structural phase transition that occurs within the primitive cell of FM TP $\mathrm{VS}_{2}$. To describe the phase transition, we plot in Fig. 4.10 several structural parameters (out-of-plane $\mathrm{S}-\mathrm{V}-\mathrm{S}$ bond angle, $\mathrm{V}-\mathrm{S}$ bond length, and out-of-plane $\mathrm{S}-\mathrm{S}$ distance) and the band gap as a function of $U$ for $\mathrm{FM} \mathrm{TP} \mathrm{VS}_{2}$. There is a sharp discontinuity in the structural parameters at $U=4 \mathrm{eV}$ that most noticeably leads to decreases in $\mathrm{S}-\mathrm{V}-\mathrm{S}$ bond angle and out-of-plane S-S distance. The band gap shows a discontinuity and begins to decrease at $U=2 \mathrm{eV}$ when the $A_{1}^{\prime}$ level is no longer the lowest unoccupied state. At $U=4 \mathrm{eV}$ there is a slight drop in band gap due to the phase transition, after which it begins to increase roughly linearly. Using the relaxed crystal structure from $U=4 \mathrm{eV}$, we are able to converge a $U=4 \mathrm{eV} \mathrm{DFT}+U$ calculation to a metastable state $6 \mathrm{meV}$ higher in energy whose electronic properties (e.g. density of states and local density matrix) resemble those of lower $U$ (i.e., $U<4 \mathrm{eV}$ ) as opposed to this new ground state. This, along with the presence of discontinuities in the structural and electronic properties, indicates that the phase transition is of first order.

To better understand the electronic aspect of the phase transition, in Fig. 4.10(e) we plot the difference in the $\mathrm{V}$ on-site density matrices (ground state minus metastable state) obtained using the same crystal structure. The most significant changes occur in the spin-up channel. Compared to the metastable state, in this spin channel the ground state has 0.16 additional occupancy of the $A_{1}^{\prime}\left(d_{3 z^{2}-r^{2}}\right)$ state and 0.16 less in total occupancy of the $E^{\prime}$ $\left(d_{x^{2}-y^{2}}\right.$ and $\left.d_{x y}\right)$ states.

Given the crude nature of $\mathrm{DFT}+U$, one must view these results with caution. More advanced calculations using DFT+DMFT, in addition to experiments, would be needed to judge the veracity of this predicted CDW. A smaller value of $U$ might be more relevant 
4. Electronic correlations in monolayer $\mathrm{VS}_{2}$

in $\mathrm{VS}_{2}$ to compensate for errors associated with Hartree-Fock treatment of the impurity problem.

\subsubsection{DFT $+U$ relative phase stability}

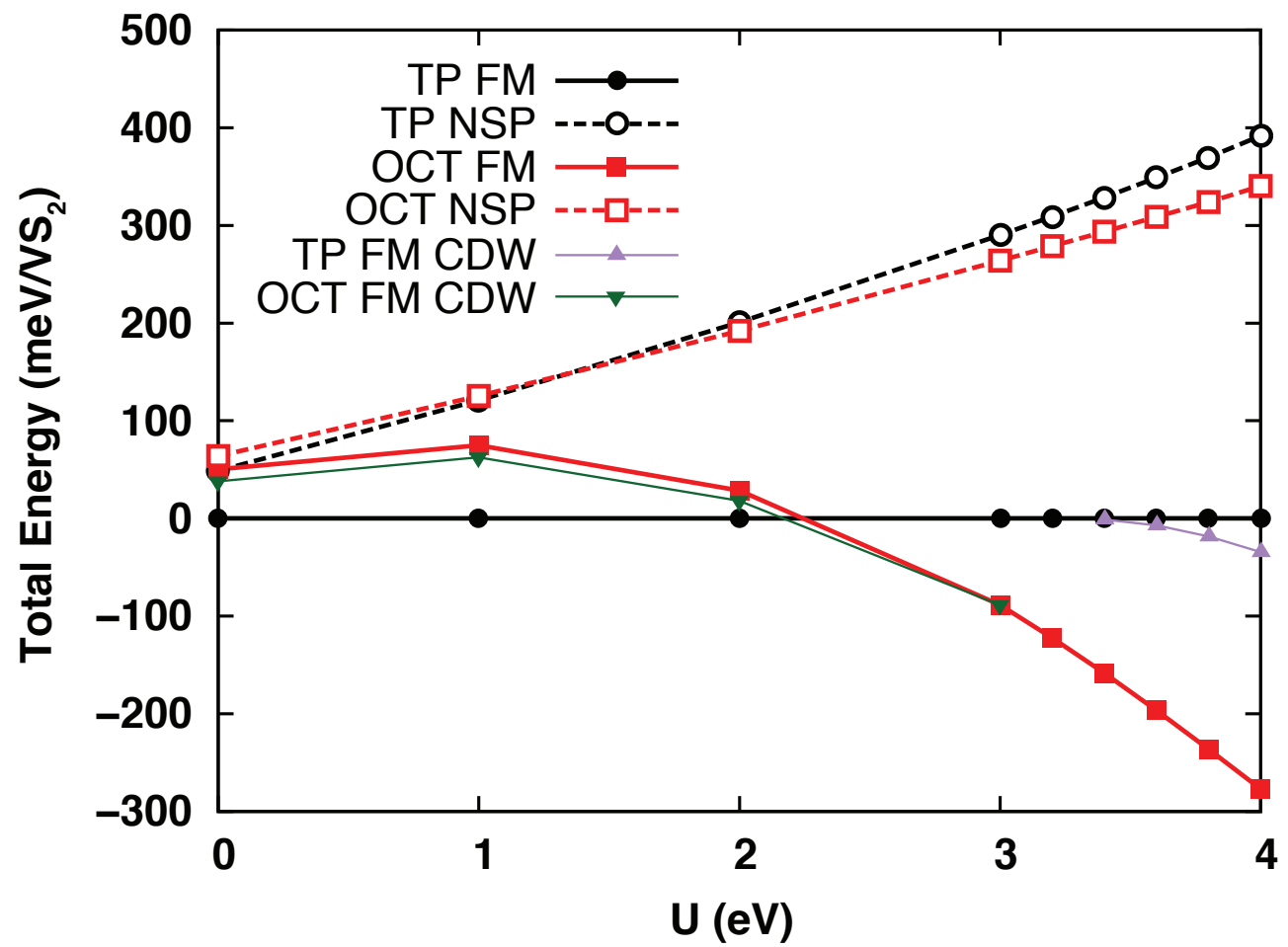

Figure 4.11: Total energy of NSP TP (black dashed line and open circles), NSP OCT (red dashed line and open squares), and FM OCT (red solid line and filled squares) states referenced to the FM TP (black solid line and filled circles) state energy as a function of $U$. The FM CDW state for the TP phase (purple solid triangles) and OCT phase (green upside-down triangles) are a small perturbation on the energetics.

To explore the impact of $U$ on the relative energetics of the TP and OCT phases, in Fig. 4.11 we show the total energy of the NSP and FM states for TP and OCT $\mathrm{VS}_{2}$ referenced to the TP FM state energy. Here we do not focus on the CDWs since they are a small perturbation on the energetics. For $U=0$ the TP FM state is the ground state with the TP 
NSP, OCT FM, and OCT NSP states 49, 50, and 64 meV higher in energy, respectively. As $U$ increases the NSP states are each monotonically destabilized by several hundreds of meV compared to the TP FM state as expected. The OCT FM phase has a more complicated nonmonotonic behavior, initially slightly increasing its relative energy with $U$ and then decreasing its relative energy for $U>1 \mathrm{eV}$. For $U$ values larger than $1 \mathrm{eV}$ the OCT FM state becomes an insulator with the $A_{1 g}$ state fully polarized (V magnetic moment of $1 \mu_{B}$ ) and is energetically stabilized; for $U=3 \mathrm{eV}$ it is lower in energy than the TP FM state by $88 \mathrm{meV}$, and the energy stabilization increases upon further increasing $U$.

To gain further insight into the stabilization of FM OCT over FM TP $\mathrm{VS}_{2}$ with $U$, we introduce a new spectral decomposition of the $\mathrm{DFT}+U$ energy functional into contributions from DFT $\left(E_{D F T}\right)$, filling of $\mathrm{V} d$ orbitals $\left(E_{f i l l}\right)$, and ordering of $\mathrm{V} d$ orbitals $\left(E_{\text {ord }}\right)$ :

$$
\begin{aligned}
& E_{D F T+U}=E_{D F T}+E_{f i l l}+E_{\text {ord }} \\
& E_{\text {fill }}=U(2 l+1) \mu(1-\mu) \quad E_{\text {ord }}=-U(2 l+1) \sigma^{2}
\end{aligned}
$$

where $l$ is the angular momentum ( $l=2$ for $d$ electrons) and $\mu$ and $\sigma$ are the mean and standard deviation of the eigenvalues of the local $d$ density matrix. The filling and ordering terms added together give the standard interaction and double counting terms in $\mathrm{DFT}+U$ for $J$ set to 0 . This decomposition provides a convenient way to isolate and quantify the contributions of the average filling of the $d$ shell and the spin and orbital ordering of the $d$ shell to the interaction and double counting energetics. The former elucidates the energetics associated with moving charge into or out of the correlated subspace, while the latter is the means by which Hartree-Fock captures the energetics of electronic correlations.

As shown in Fig. 4.12(a), for $U=1 \mathrm{eV} E_{D F T}$ (black circles) and $E_{\text {ord }}$ (blue diamonds) are responsible for the further stabilization of the TP phase compared to $U=0$. For larger $U$, the $E_{\text {fill }}$ term (green triangles) increasingly favors the OCT phase by as much as 101 meV as $U$ increases. The total $\mathrm{E}(\mathrm{OCT})-\mathrm{E}(\mathrm{TP})$ (red squares) decreases with $U$ a factor of 3 to 4 faster than $E_{f i l l}$. $E_{D F T}$ and $E_{\text {ord }}$ tend to oppose each other, but overall the negative 


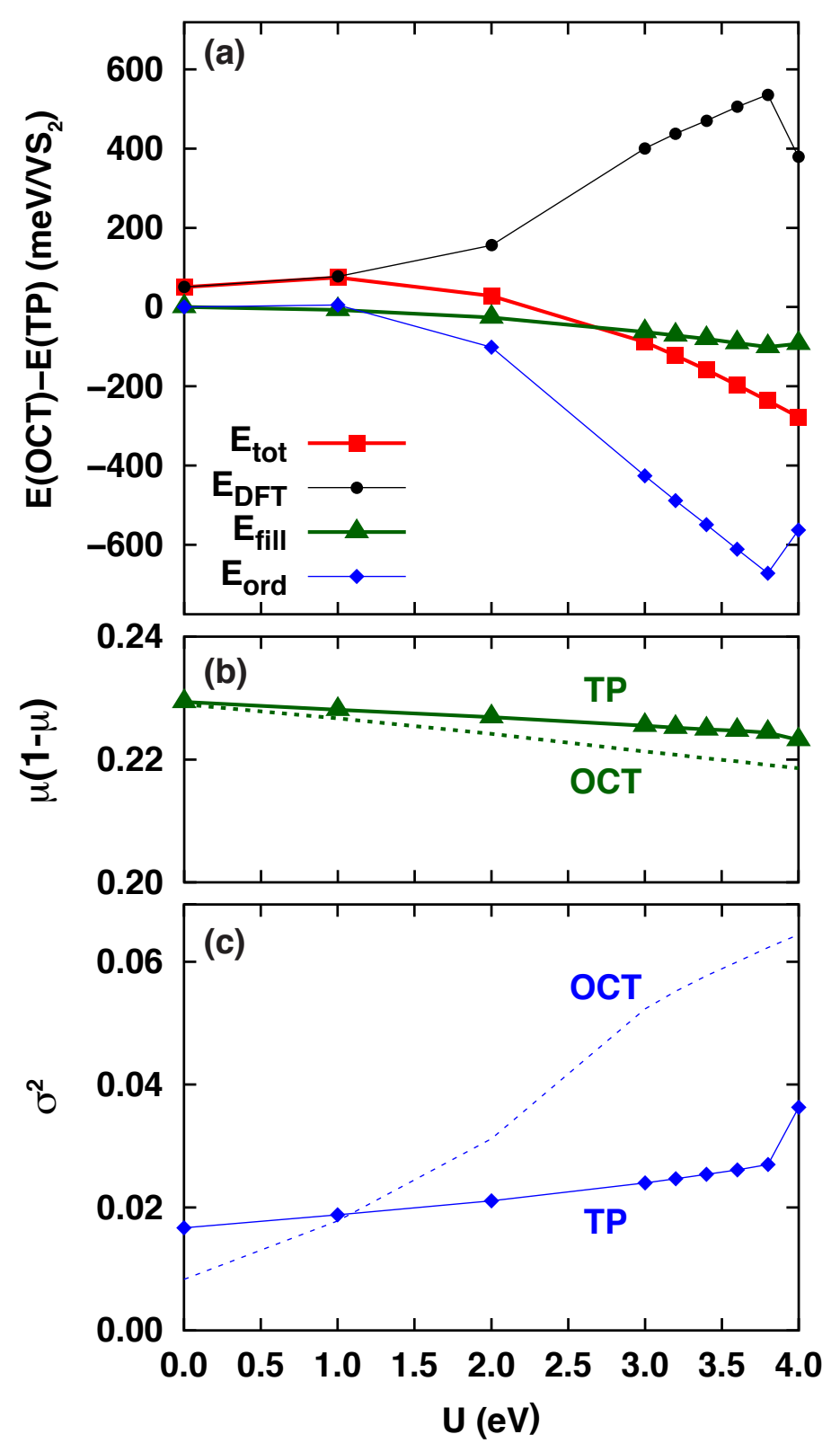

Figure 4.12: (a) DFT $+U$ total energy of FM OCT phase minus that of FM TP phase (red squares) and decomposition into DFT (black circles), filling (green triangles), and ordering (blue diamonds) contributions as a function of $U$. (b) $\mu(1-\mu)$ (green) and (c) $\sigma^{2}$ (blue) as a function of $U$. Solid (dashed) lines with (without) symbols correspond to the TP (OCT) phase in panels (b) and (c). 
$E_{\text {ord }}$ term is dominant and this contributes significantly to the overall stabilization of the OCT phase. The $E_{\text {ord }}$ and $E_{D F T}$ terms increase in magnitude significantly faster once the OCT phase becomes an insulator at $U=2 \mathrm{eV}$. We find the same qualitative behavior when we freeze the ions at the $U=0$ structures, indicating this is not an effect of structural relaxation.

The filling factor $\mu(1-\mu)$ and the ordering factor $\sigma^{2}$ are plotted for both phases in Fig. 4.12(b) and Fig. 4.12(c), respectively. Interestingly, the TP and OCT phases have an almost identical filling of the $\mathrm{V} d$ shell with $\mu(1-\mu)=0.229$ at $U=0$. On the other hand, the $\sigma^{2}$ terms are substantially different at $U=0: \sigma^{2}$ is 0.0167 in the TP phase as opposed to only 0.0083 in the OCT phase. This stems from the complete spin polarization of the $A_{1}^{\prime}$ state in the TP phase, as opposed to the partial spin polarization in the OCT phase. The preceding statement can be supported by investigating the NSP state for both the TP and OCT phases for $U=0$, which yields much more similar $\sigma^{2}$ values of 0.0037 and 0.0047 , respectively. Therefore, the pure crystal fields in each respective case results in a similar and small $\sigma^{2}$, while the differing degrees of spin polarization are responsible for the large initial difference at $U=0$. This enhanced spin ordering in the TP phase leads to the enhanced stabilization of the TP phase in the limit of small $U$ since $\partial E_{\text {ord }} / \partial U \sim-\sigma^{2}$ and because the initial fillings are nearly identical. However, this trend is only guaranteed for small $U$ and as we pointed out above the trend reverses for $U>1 \mathrm{eV}$. We therefore proceed to examine each contribution as a function of $U$. In terms of the filling contribution, the OCT phase filling factor decreases with $U$ twice as fast as it does for the TP phase for $U \leq 3.8 \mathrm{eV}$. The $\sigma^{2}$ for the OCT phase increases 5.2 times as fast as does that of the TP phase for $U \leq 3.8 \mathrm{eV}$, since both the $A_{1 g}$ and the $E_{g}^{\prime}$ states are polarizable, and for $U=3.8 \mathrm{eV}$ it has an ordering factor 2.3 times as large. Therefore, both the decreased filling and increased ordering of the $d$ orbitals of the OCT phase contribute to its stabilization for larger $U$. 


\subsubsection{Possibility of realizing trigonal prismatic $\mathrm{VS}_{2}$}

Only the OCT phase of $\mathrm{VS}_{2}$ has been observed experimentally, in bulk and nanosheet forms [Murphy et al. (1977); Feng et al. (2011, 2012); Zhong et al. (2014); Gauzzi et al. (2014)]. DFT predicts the TP phase is the thermodynamic ground state, while DFT $+U$ predicts that the OCT phase becomes the ground state when $U$ surpasses a moderate value of approximately $2.3 \mathrm{eV}$. More advanced calculations, including DFT+DMFT and possibly cluster extensions of DMFT, will be needed to definitively settle this issue from a theoretical standpoint. Given that TP may in fact be the ground state, or possibly a metastable state sufficiently low in energy to be achieved experimentally, we explore possible reasons why it has not been observed in experiment.

The initial synthetic route to $\mathrm{VS}_{2}$ was delithiation from $\mathrm{LiVS}_{2}$ [Murphy et al. (1977)]. This lithiated compound has a layered octahedral structure [van Laar and Ijdo (1971)]. Therefore, one possibility is that $\mathrm{VS}_{2}$ is stuck in a metastable OCT state. Within DFT, we compute an energy barrier of $0.69 \mathrm{eV}$ per formula unit based on a linear interpolation between the TP and OCT monolayer structures allowing only out-of-plane ionic relaxation. This value is in agreement with nudged elastic band calculations that found a barrier of $0.66 \mathrm{eV}$ [Zhang et al. (2013)]. The large barrier supports the possibility that it could very

challenging to change phases. Another high-temperature synthesis technique did not use $\mathrm{LiVS}_{2}$ but still resulted in the OCT phase [Ohno et al. $\left.(1982,1983)\right]$. One possibility is that finite temperature plays a role in destabilizing the TP phase since there is evidence that the phonon entropy is greater for the OCT phase [Zhang et al. (2013)].

A more recent high-pressure synthesis of $\mathrm{VS}_{2}$ also yielded the OCT phase [Gauzzi et al. (2014)]. We performed spin-polarized DFT (i.e., $U=0$ ) calculations of bulk $\mathrm{VS}_{2}$ under pressure and find that for sufficiently high pressure the OCT phase becomes the ground state, so this could be the reason why the TP phase is not observed. In these calculations we considered $2 \mathrm{H}_{c}\left(\mathrm{MoS}_{2}\right.$-like) stacking [Katzke et al. (2004)] for the TP phase and O1 $\left(\mathrm{CoO}_{2^{-}}\right.$ like) and $\mathrm{O} 3\left(\mathrm{LiCoO}_{2}\right.$-like) stackings [Van der Ven et al. (1998)] for the OCT phase. At 5 
GPa the TP phase is still the ground state but only $15 \mathrm{meV}$ lower in energy compared to $50 \mathrm{meV}$ for $0 \mathrm{GPa}$. At $10 \mathrm{GPa}$ the $\mathrm{TP}$ phase becomes $26 \mathrm{meV}$ higher in energy than the OCT phase. Based on these observations, if the TP phase is the ground state we predict that synthesis under ambient pressure, under low temperature, and not involving a $\mathrm{LiVS}_{2}$ precursor will be most effective to attempt to realize $\mathrm{TP} \mathrm{VS}_{2}$.

\subsection{Conclusions}

We have demonstrated that monolayer $\mathrm{TP} \mathrm{VS}_{2}$ has an isolated low-energy band at level of NSP DFT, which arises due to a combination of the TP crystal field and the nearest-neighbor $\mathrm{V}-\mathrm{V}$ hopping. Including spin polarization reveals that the exchange is ferromagnetic and yields a FM insulator with a small band gap. Other spin configurations result in metallic states substantially higher in energy, indicating that spin-dependent DFT is not putting $\mathrm{VS}_{2}$ in the Mott regime. While TP $\mathrm{VS}_{2}$ has not been observed in experiment in any form, spin-polarized DFT does predict it is lower in energy than the OCT phase. DFT captures the known CDW in the OCT phase, which strongly diminishes the magnetism relative to the undistorted phase. However, DFT appears to grossly overestimate the CDW amplitude in this phase. Specifically, the $\mathrm{V}-\mathrm{V}$ distance differences from DFT are far larger than those of the existing XAFS study [Sun et al. (2015)].

Accounting for local correlations via $\mathrm{DFT}+U$ produces a $S=1 / 2 \mathrm{FM}$ insulating state in the TP phase, which is in the Mott regime for moderate values of $U$. For a small regime of finite $U$, we find a CDW in the TP phase at $q=3 / 5 K$. For the OCT phase, increasing $U$ diminishes the amplitude of the CDW. For the ferromagnetic CDW state, the amplitude decreases slowly before rapidly collapsing near $U=3 \mathrm{eV}$. However, for this regime of $U$, magnetism with anti-aligned spins becomes energetically favored over ferromagnetism. In this magnetic configuration we find metallic behavior as in experiments and the $\mathrm{V}-\mathrm{V}$ distance differences of the CDW phase are within reasonable comparison to XAFS experiments. 
Regarding relative phase stability, above a reasonably small $U$ (approx. $2.3 \mathrm{eV}$ ) the energy ordering of TP and OCT phases reverses with the OCT phase becoming the ground state. More advanced calculations, including DFT+DMFT and possibly cluster extensions of DMFT, will be needed to settle which is the ground state structure and determine whether the CDW in the TP phase is physical.

If the TP phase can be realized, it has the potential for novel physics: it would be a rare example of a $S=1 / 2$ Mott insulator on a triangular lattice with strong FM correlations. Its monolayer nature might enable doping via gating, allowing one to probe the doped Mott insulator in a precise fashion without simultaneously introducing disorder.

\subsection{Acknowledgments}

This research used resources of the National Energy Research Scientific Computing Center, a DOE Office of Science User Facility supported by the Office of Science of the U.S. Department of Energy under Contract No. DE-AC02-05CH11231. The authors acknowledge support from the NSF MRSEC program through Columbia University in the Center for Precision Assembly of Superstratic and Superatomic Solids (DMR-1420634). E.B.I. gratefully acknowledges support from the U.S. Department of Energy Computational Science Graduate Fellowship (Grant No. DE-FG02-97ER25308). 


\section{Bibliography}

Amatucci, G. G., Tarascon, J. M. and Klein, L. C., $\mathrm{CoO}_{2}$, The End Member of the $\mathrm{Li}_{x} \mathrm{CoO}_{2}$ Solid Solution, J. Electrochem. Soc. 143, 1114 (1996).

Amine, K., Yasuda, H. and Yamachi, M., Olivine $\mathrm{LiCoPO}_{4}$ as $4.8 \mathrm{~V}$ Electrode Material for Lithium Batteries, Electrochem. Solid-State Lett. 3, 178 (2000).

Anderson, P. W., Localized Magnetic States in Metals, Phys. Rev. 124, 41 (1961).

Andriyevsky, B., Doll, K. and Jacob, T., Electronic and transport properties of $\mathrm{LiCoO}_{2}$, Phys. Chem. Chem. Phys. 16, 23412 (2014).

Anisimov, V. I., Aryasetiawan, F. and Lichtenstein, A. I., First-principles calculations of the electronic structure and spectra of strongly correlated systems: the LDA+U method, J. Phys.: Condens. Matter 9, 767 (1997).

Anisimov, V. I., Solovyev, I. V., Korotin, M. A., Czyżyk, M. T. and Sawatzky, G. A., Densityfunctional theory and $\mathrm{NiO}$ photoemission spectra, Phys. Rev. B 48, 16929 (1993).

Anisimov, V. I., Zaanen, J. and Andersen, O. K., Band theory and Mott insulators: Hubbard U instead of Stoner I, Phys. Rev. B 44, 943 (1991).

Aydinol, M. K., Kohan, A. F., Ceder, G., Cho, K. and Joannopoulos, J., Ab initio study of lithium intercalation in metal oxides and metal dichalcogenides, Phys. Rev. B 56, 1354 (1997).

Aykol, M., Kim, S. and Wolverton, C., van der Waals Interactions in Layered Lithium Cobalt Oxides, J. Phys. Chem. C 119, 19053 (2015). 
Aykol, M. and Wolverton, C., Local environment dependent GGA $+U$ method for accurate thermochemistry of transition metal compounds, Phys. Rev. B 90, 115105 (2014).

Bednorz, J. G. and Müller, K. A., Possible high $\mathrm{T}_{c}$ superconductivity in the BaLaCuO system, Z. Phys. B 64, 189 (1986).

Blöchl, P. E., Projector augmented-wave method, Phys. Rev. B 50, 17953 (1994).

Blöchl, P. E., Jepsen, O. and Andersen, O. K., Improved tetrahedron method for Brillouinzone integrations, Phys. Rev. B 49, 16223 (1994).

Bramnik, N. N., Nikolowski, K., Baehtz, C., Bramnik, K. G. and Ehrenberg, H., Phase Transitions Occurring upon Lithium Insertion-Extraction of $\mathrm{LiCoPO}_{4}$, Chem. Mater. 19, $908(2007)$.

Cable, J. W., Wilkinson, M. K. and Wollan, E. O., Neutron diffraction investigation of antiferromagnetism in $\mathrm{CrCl}_{3}$, J. Phys. Chem. Solids 19, 29 (1961).

Carteaux, V., Brunet, D., Ouvrard, G. and Andre, G., Crystallographic, magnetic and electronic structures of a new layered ferromagnetic compound $\mathrm{Cr}_{2} \mathrm{Ge}_{2} \mathrm{Te}_{6}$, J. Phys.: Condens. Matter 7, 69 (1995).

Carteaux, V., Ouvrard, G., Grenier, J. C. and Laligant, Y., Magnetic structure of the new layered ferromagnetic chromium hexatellurosilicate $\mathrm{Cr}_{2} \mathrm{Si}_{2} \mathrm{Te}_{6}$, J. Magn. Magn. Mater. 94, 127 (1991).

Ceder, G., Opportunities and challenges for first-principles materials design and applications to Li battery materials, Mater. Res. Bull. 35, 693 (2010).

Ceperley, D. M. and Alder, B. J., Ground State of the Electron Gas by a Stochastic Method, Phys. Rev. Lett. 45, 566 (1980). 
Chen, B., Yang, J., Wang, H., Imai, M., Ohta, H., Michioka, C., Yoshimura, K. and Fang, M., Magnetic Properties of Layered Itinerant Electron Ferromagnet $\mathrm{Fe}_{3} \mathrm{GeTe}_{2}$, J. Phys. Soc. Jpn. 82, 124711 (2013).

Chen, J., Millis, A. J. and Marianetti, C. A., Density functional plus dynamical mean-field theory of the spin-crossover molecule Fe(phen $)_{2}(\mathrm{NCS})_{2}$, Phys. Rev. B 91, 241111 (2015).

Chevrier, V. L., Ong, S. P., Armiento, R., Chan, M. K. Y. and Ceder, G., Hybrid density functional calculations of redox potentials and formation energies of transition metal compounds, Phys. Rev. B 82, 075122 (2010).

Cococcioni, M. and de Gironcoli, S., Linear response approach to the calculation of the effective interaction parameters in the LDA+U method, Phys. Rev. B 71, 035105 (2005).

Coleman, P., Heavy Fermions: Electrons at the Edge of Magnetism, in Handbook of Magnetism and Advanced Magnetic Materials, John Wiley \& Sons, Ltd. (2007).

Czyżyk, M. T., Potze, R. and Sawatzky, G. A., Band-theory description of high-energy spectroscopy and the electronic structure of $\mathrm{LiCoO}_{2}$, Phys. Rev. B 46, 3729 (1992).

Dai, P., Antiferromagnetic order and spin dynamics in iron-based superconductors, Rev. Mod. Phys. 87, 855 (2015).

Darancet, P., Millis, A. J. and Marianetti, C. A., Three-dimensional metallic and twodimensional insulating behavior in octahedral tantalum dichalcogenides, Phys. Rev. B 90, 045134 (2014).

de Haas, W. J., Schultz, B. H. and Koolhaas, J., Further measurements of the magnetic properties of some salts of the iron group at low temperatures, Physica 7, 57 (1940).

de Vaulx, C., Julien, M.-H., Berthier, C., Hébert, S., Pralong, V. and Maignan, A., Electronic Correlations in $\mathrm{CoO}_{2}$, the Parent Compound of Triangular Cobaltates, Phys. Rev. Lett. 98, 246402 (2007). 
Deiseroth, H.-J., Aleksandrov, K., Reiner, C., Kienle, L. and Kremer, R. K., Fe $\mathrm{FeTe}_{2}$ and $\mathrm{Ni}_{3} \mathrm{GeTe}_{2}$ Two New Layered Transition-Metal Compounds: Crystal Structures, HRTEM Investigations, and Magnetic and Electrical Properties, Eur. J. Inorg. Chem. 2006, 1561 (2006).

Delacourt, C., Poizot, P., Tarascon, J.-M. and Masquelier, C., The existence of a temperature-driven solid solution in $\mathrm{Li}_{x} \mathrm{FePO}_{4}$ for $0 \leq x \leq 1$, Nat. Mater. 4, 254 (2005).

Dillon Jr., J. F. and Olson, C. E., Magnetization, Resonance, and Optical Properties of the Ferromagnet $\mathrm{CrI}_{3}$, J. App. Phys. 36, 1259 (1965).

Dirac, P. A. M., Quantum Mechanics of Many-Electron Systems, Proceedings of the Royal Society of London. Series A, Containing Papers of a Mathematical and Physical Character 123, 714 (1929).

Dodd, J. L., Yazami, R. and Fultz, B., Phase Diagram of $\mathrm{Li}_{x} \mathrm{FePO}_{4}$, Electrochem. Solid-State Lett. 9, A151 (2006).

Dudarev, S. L., Botton, G. A., Savrasov, S. Y., Humphreys, C. J. and Sutton, A. P., Electronenergy-loss spectra and the structural stability of nickel oxide:An LSDA+U study, Phys. Rev. B 57, 1505 (1998).

Ehrenberg, H., Bramnik, N. N., Senyshyn, A. and Fuess, H., Crystal and magnetic structures of electrochemically delithiated $\mathrm{Li}_{1 x} \mathrm{CoPO}_{4}$ phases, Solid State Sci. 11, 18 (2009).

Ellis, B., Perry, L. K., Ryan, D. H. and Nazar, L. F., Small Polaron Hopping in $\mathrm{Li}_{x} \mathrm{FePO}_{4}$ Solid Solutions: Coupled Lithium-Ion and Electron Mobility, J. Am. Chem. Soc. 128, 11416 (2006).

Ellis, B. L., Lee, K. T. and Nazar, L. F., Positive Electrode Materials for Li-Ion and LiBatteries, Chem. Mater. 22, 691 (2010). 
Feng, J., Peng, L., Wu, C., Sun, X., Hu, S., Lin, C., Dai, J., Yang, J. and Xie, Y., Giant Moisture Responsiveness of $\mathrm{VS}_{2}$ Ultrathin Nanosheets for Novel Touchless Positioning Interface, Adv. Mater. 24, 1969 (2012).

Feng, J., Sun, X., Wu, C., Peng, L., Lin, C., Hu, S., Yang, J. and Xie, Y., Metallic FewLayered $\mathrm{VS}_{2}$ Ultrathin Nanosheets: High Two-Dimensional Conductivity for In-Plane Supercapacitors, J. Am. Chem. Soc. 133, 17832 (2011).

Gao, D., Xue, Q., Mao, X., Wang, W., Xu, Q. and Xue, D., Ferromagnetism in ultrathin $\mathrm{VS}_{2}$ nanosheets, J. Mater. Chem. C 1, 5909 (2013).

Gauzzi, A., Sellam, A., Rousse, G., Klein, Y., Taverna, D., Giura, P., Calandra, M., Loupias, G., Gozzo, F., Gilioli, E., Bolzoni, F., Allodi, G., De Renzi, R., Calestani, G. L. and Roy, P., Possible phase separation and weak localization in the absence of a charge-density wave in single-phase $1 \mathrm{~T}-\mathrm{VS}_{2}$, Phys. Rev. B 89, 235125 (2014).

Georges, A. and Kotliar, G., Hubbard model in infinite dimensions, Phys. Rev. B 45, 6479 (1992).

Georges, A., Kotliar, G., Krauth, W. and Rozenberg, M. J., Dynamical mean-field theory of strongly correlated fermion systems and the limit of infinite dimensions, Rev. Mod. Phys. 68, 13 (1996).

Goodenough, J. B., Theory of the Role of Covalence in the Perovskite-Type Manganites [La, $M(\mathrm{II})] \mathrm{MnO}_{3}$, Phys. Rev. 100, 564 (1955).

Goodenough, J. B., An interpretation of the magnetic properties of the perovskite-type mixed crystals $\mathrm{La}_{1-x} \mathrm{Sr}_{x} \mathrm{CoO}_{3-\lambda}$, J. Phys. Chem. Solids 6, 287 (1958).

Gull, E., Millis, A. J., Lichtenstein, A. I., Rubtsov, A. N., Troyer, M. and Werner, P., Continuous-time MonteCarlo methods for quantum impurity models, Rev. Mod. Phys. 83, 349 (2011). 
Gutzwiller, M. C., Effect of Correlation on the Ferromagnetism of Transition Metals, Phys. Rev. Lett. 10, 159 (1963).

Haule, K., Quantum Monte Carlo impurity solver for cluster dynamical mean-field theory and electronic structure calculations with adjustable cluster base, Phys. Rev. B 75, 155113 (2007).

Hautier, G., Jain, A., Chen, H., Moore, C., Ong, S. P. and Ceder, G., Novel mixed polyanions lithium-ion battery cathode materials predicted by high-throughput ab initio computations, J. Mater. Chem. 21, 17147 (2011).

Hautier, G., Jain, A., Ong, S. P., Kang, B., Moore, C., Doe, R. and Ceder, G., Phosphates as Lithium-Ion Battery Cathodes: An Evaluation Based on High-Throughput ab Initio Calculations, Chem. Mater. 23, 3495 (2011).

Himmetoglu, B., Floris, A., de Gironcoli, S. and Cococcioni, M., Hubbard-corrected DFT energy functionals: The LDA+U description of correlated systems, Int. J. Quant. Chem. 114, 14 (2014).

Hohenberg, P. and Kohn, W., Inhomogeneous Electron Gas, Phys. Rev. 136, B864 (1964).

Huang, P.-R., He, Y., Pal, H. K. and Kindermann, M., Prediction of switchable half semiconductor in $\mathrm{d}^{1}$ transition metal dichalcogenide monolayers, arXiv:1501.00760 .

Hubbard, J., Electron Correlations in Narrow Energy Bands, Proc. R. Soc. Lond. A 276, 238 (1963).

Imada, M., Fujimori, A. and Tokura, Y., Metal-insulator transitions, Rev. Mod. Phys. 70, 1039 (1998).

Jeevanandam, P. and Vasudevan, S., Magnetism in $\mathrm{MnPSe}_{3}$ : a layered $3 \mathrm{~d}^{5}$ antiferromagnet with unusually large XY anisotropy, J. Phys.: Condens. Matter 11, 3563 (1999). 
Jiang, C., Sordelet, D. J. and Gleeson, B., First-principles study of phase stability in pseudobinary $\left(\mathrm{Ni}_{1-x} \mathrm{Pt}_{x}\right)_{3} \mathrm{Al}$ alloys, Phys. Rev. B 72, 184203 (2005).

Jiang, C., Stanek, C. R., Sickafus, K. E. and Uberuaga, B. P., First-principles prediction of disordering tendencies in pyrochlore oxides, Phys. Rev. B 79, 104203 (2009).

Jiang, C., Wolverton, C., Sofo, J., Chen, L.-Q. and Liu, Z.-K., First-principles study of binary bcc alloys using special quasirandom structures, Phys. Rev. B 69, 214202 (2004).

Johnston, W. D., Heikes, R. R. and Sestrich, D., The preparation, crystallography, and magnetic properties of the $\mathrm{Li}_{x} \mathrm{Co}_{1 x} \mathrm{O}$ system, J. Phys. Chem. Solids 7, 1 (1958).

Jones, R., Density functional theory: Its origins, rise to prominence, and future, Rev. Mod. Phys. 87, 897 (2015).

Jones, R. O. and Gunnarsson, O., The density functional formalism, its applications and prospects, Rev. Mod. Phys. 61, 689 (1989).

Kanamori, J., Superexchange interaction and symmetry properties of electron orbitals, J. Phys. Chem. Solids 10, 87 (1959).

Kanamori, J., Electron Correlation and Ferromagnetism of Transition Metals, Prog. Theor. Phys. 30, 275 (1963).

Katzke, H., Tolédano, P. and Depmeier, W., Phase transitions between polytypes and intralayer superstructures in transition metal dichalcogenides, Phys. Rev. B 69, 134111 (2004).

Kawasaki, S., Motohashi, T., Shimada, K., Ono, T., Kanno, R., Karppinen, M., Yamauchi, H. and Zheng, G.-q., Measurement of electron correlations in $\mathrm{Li}_{x} \mathrm{CoO}_{2}(x=0.0-0.35)$ using ${ }^{59}$ Co nuclear magnetic resonance and nuclear quadrupole resonance techniques, Phys. Rev. B 79, 220514 (2009). 
Kertesz, M. and Hoffmann, R., Octahedral vs. trigonal-prismatic coordination and clustering in transition-metal dichalcogenides, J. Am. Chem. Soc. 106, 3453 (1984).

Kittel, C., Introduction to Solid State Physics, 6th ed., John Wiley \& Sons, Inc., New York (1986).

Kohn, W. and Sham, L. J., Self-Consistent Equations Including Exchange and Correlation Effects, Phys. Rev. 140, A1133 (1965).

Kotliar, G., Savrasov, S. Y., Haule, K., Oudovenko, V. S., Parcollet, O. and Marianetti, C. A., Electronic structure calculations with dynamical mean-field theory, Rev. Mod. Phys. 78, 865 (2006).

Kotliar, G. and Vollhardt, D., Strongly correlated materials: Insights from dynamical meanfield theory, Phys. Today 57, 53 (2004).

Kresse, G. and Furthmüller, J., Efficiency of ab-initio total energy calculations for metals and semiconductors using a plane-wave basis set, Comput. Mater. Sci. 6, 15 (1996).

Kresse, G. and Furthmüller, J., Efficient iterative schemes for ab initio total-energy calculations using a plane-wave basis set, Phys. Rev. B 54, 11169 (1996).

Kresse, G. and Hafner, J., Ab initio molecular dynamics for liquid metals, Phys. Rev. B 47, $558(1993)$.

Kresse, G. and Hafner, J., Ab initio molecular-dynamics simulation of the liquidmetalamorphous-semiconductor transition in germanium, Phys. Rev. B 49, 14251 (1994).

Kresse, G. and Joubert, D., From ultrasoft pseudopotentials to the projector augmentedwave method, Phys. Rev. B 59, 1758 (1999).

Lebègue, S., Björkman, T., Klintenberg, M., Nieminen, R. M. and Eriksson, O., TwoDimensional Materials from Data Filtering and Ab Initio Calculations, Phys. Rev. X 3, 031002 (2013). 
$\mathrm{Li}, \mathrm{X}$. and Yang, J., $\mathrm{CrXTe}_{3}(\mathrm{X}=\mathrm{Si}, \mathrm{Ge})$ nanosheets: two dimensional intrinsic ferromagnetic semiconductors, J. Mater. Chem. C 2, 7071 (2014).

Liechtenstein, A. I., Anisimov, V. I. and Zaanen, J., Density-functional theory and strong interactions: Orbital ordering in Mott-Hubbard insulators, Phys. Rev. B 52, R5467 (1995).

Lu, Z. W., Laks, D. B., Wei, S.-H. and Zunger, A., First-principles simulated-annealing study of phase transitions and short-range order in transition-metal and semiconductor alloys, Phys. Rev. B 50, 6642 (1994).

Maier, T., Jarrell, M., Pruschke, T. and Hettler, M. H., Quantum cluster theories, Rev. Mod. Phys. 77, 1027 (2005).

Marder, M., Papanicolaou, N. and Psaltakis, G. C., Phase separation in a t-J model, Phys. Rev. B 41, 6920 (1990).

Marianetti, C. A., Kotliar, G. and Ceder, G., Role of Hybridization in $\mathrm{Na}_{x} \mathrm{CoO}_{2}$ and the Effect of Hydration, Phys. Rev. Lett. 92, 196405 (2004).

Marzari, N. and Vanderbilt, D., Maximally localized generalized Wannier functions for composite energy bands, Phys. Rev. B 56, 12847 (1997).

Meethong, N., Huang, H.-Y. S., Carter, W. C. and Chiang, Y.-M., Size-Dependent Lithium Miscibility Gap in Nanoscale $\mathrm{Li}_{1 x} \mathrm{FePO}_{4}$, Electrochem. Solid-State Lett. 10, A134 (2007).

Ménétrier, M., Carlier, D., Blangero, M. and Delmas, C., On Really Stoichiometric $\mathrm{LiCoO}_{2}$, Electrochem. Solid-State Lett. 11, A179 (2008).

Ménétrier, M., Saadoune, I., Levasseur, S. and Delmas, C., The insulator-metal transition upon lithium deintercalation from $\mathrm{LiCoO}_{2}$ : electronic properties and ${ }^{7} \mathrm{Li} \mathrm{NMR}$ study, J. Mater. Chem. 9, 1135 (1999). 
Methfessel, M. and Paxton, A. T., High-precision sampling for Brillouin-zone integration in metals, Phys. Rev. B 40, 3616 (1989).

Metzner, W. and Vollhardt, D., Correlated Lattice Fermions in d= Dimensions, Phys. Rev. Lett. 62, 324 (1989).

Miyoshi, K., Iwai, C., Kondo, H., Miura, M., Nishigori, S. and Takeuchi, J., Magnetic and electronic properties of $\mathrm{Li}_{x} \mathrm{CoO}_{2}$ single crystals, Phys. Rev. B 82, 075113 (2010).

Mizokawa, T., Wakisaka, Y., Sudayama, T., Iwai, C., Miyoshi, K., Takeuchi, J., Wadati, H., Hawthorn, D. G., Regier, T. Z. and Sawatzky, G. A., Role of Oxygen Holes in $\mathrm{Li}_{x} \mathrm{CoO}_{2}$ Revealed by Soft X-Ray Spectroscopy, Phys. Rev. Lett. 111, 056404 (2013).

Mizushima, K., Jones, P., Wiseman, P. and Goodenough, J., $\mathrm{Li}_{x} \mathrm{CoO}_{2}(0<x<1)$ : A new cathode material for batteries of high energy density, Mater. Res. Bull. 15, 783 (1980).

Momma, K. and Izumi, F., VESTA 3 for three-dimensional visualization of crystal, volumetric and morphology data, J. Appl. Crystallogr. 44, 1272 (2011).

Morosan, E., Natelson, D., Nevidomskyy, A. H. and Si, Q., Strongly Correlated Materials, Adv. Mater. 24, 4896 (2012).

Mostofi, A. A., Yates, J. R., Lee, Y.-S., Souza, I., Vanderbilt, D. and Marzari, N., wannier90: A tool for obtaining maximally-localised Wannier functions, Comput. Phys. Commun. 178, 685 (2008).

Motohashi, T., Katsumata, Y., Ono, T., Kanno, R., Karppinen, M. and Yamauchi, H., Synthesis and Properties of $\mathrm{CoO}_{2}$, the $x=0$ End Member of the $\mathrm{Li}_{x} \mathrm{CoO}_{2}$ and $\mathrm{Na}_{x} \mathrm{CoO}_{2}$ Systems, Chem. Mater. 19, 5063 (2007).

Motohashi, T., Ono, T., Sugimoto, Y., Masubuchi, Y., Kikkawa, S., Kanno, R., Karppinen, M. and Yamauchi, H., Electronic phase diagram of the layered cobalt oxide system $\mathrm{Li}_{x} \mathrm{CoO}_{2}(0.0 \leq x \leq 1.0)$, Phys. Rev. B 80, 165114 (2009). 
Mott, N. F., Metal-Insulator Transition, Rev. Mod. Phys. 40, 677 (1968).

Mueller, T., Hautier, G., Jain, A. and Ceder, G., Evaluation of Tavorite-Structured Cathode Materials for Lithium-Ion Batteries Using High-Throughput Computing, Chem. Mater. 23, 3854 (2011).

Mulazzi, M., Chainani, A., Katayama, N., Eguchi, R., Matsunami, M., Ohashi, H., Senba, Y., Nohara, M., Uchida, M., Takagi, H. and Shin, S., Absence of nesting in the chargedensity-wave system $1 \mathrm{~T}-\mathrm{VS}_{2}$ as seen by photoelectron spectroscopy, Phys. Rev. B 82, $075130(2010)$.

Müller-Hartmann, E., Correlated fermions on a lattice in high dimensions, Z. Phys. B Cond. Mat. 74, 507 (1989).

Murphy, D. W., Cros, C., Di Salvo, F. J. and Waszczak, J. V., Preparation and properties of $\mathrm{Li}_{x} \mathrm{VS}_{2}(0 \leq x \leq 1)$, Inorg. Chem. 16, 3027 (1977).

Novoselov, K. S., Jiang, D., Schedin, F., Booth, T. J., Khotkevich, V. V., Morozov, S. V. and Geim, A. K., Two-dimensional atomic crystals, Proc. Nat. Acad. Sci. U.S.A. 102, $10451(2005)$.

Ohkawa, F. J., Electron Correlation in the Hubbard Model in $d=\infty$ Dimension, J. Phys. Soc. Jpn. 60, 3218 (1991).

Ohkawa, F. J., Heavy Electrons in the Mott-Transition Region, Prog. Theor. Phys. Supplement 106, 95 (1991).

Ohno, Y., Hirama, K., Nakai, S., Sugiura, C. and Okada, S., X-ray absorption spectroscopy of layer transition-metal disulfides, Phys. Rev. B 27, 3811 (1983).

Ohno, Y., Watanabe, H., Kawata, A., Nakai, S. and Sugiura, C., X-ray-absorption spectra and electronic structures of vanadium dichalcogenides and their first-row transition-metal intercalates, Phys. Rev. B 25, 815 (1982). 
Ong, S. P., Chevrier, V. L. and Ceder, G., Comparison of small polaron migration and phase separation in olivine $\mathrm{LiMnPO}_{4}$ and $\mathrm{LiFePO}_{4}$ using hybrid density functional theory, Phys. Rev. B 83, 075112 (2011).

Orman, H. J. and Wiseman, P. J., Cobalt(III) lithium oxide, $\mathrm{CoLiO}_{2}$ : structure refinement by powder neutron diffraction, Acta Crystallogr. Sect. C 40, 12 (1984).

Ou-Yang, T. Y., Huang, F.-T., Shu, G. J., Lee, W. L., Chu, M.-W., Liu, H. L. and Chou, F. C., Electronic phase diagram of $\mathrm{Li}_{x} \mathrm{CoO}_{2}$ revisited with potentiostatically deintercalated single crystals, Phys. Rev. B 85, 035120 (2012).

Padhi, A. K., Nanjundaswamy, K. S. and Goodenough, J. B., Effect of Structure on the $\mathrm{Fe}^{3+} / \mathrm{Fe}^{2+}$ Redox Couple in Iron Phosphates, J. Electrochem. Soc. 144, 1609 (1997).

Padhi, A. K., Nanjundaswamy, K. S. and Goodenough, J. B., Phospho-olivines as PositiveElectrode Materials for Rechargeable Lithium Batteries, J. Electrochem. Soc. 144, 1188 (1997).

Park, H., Millis, A. J. and Marianetti, C. A., Computing total energies in complex materials using charge self-consistent DFT + DMFT, Phys. Rev. B 90, 235103 (2014).

Park, H., Millis, A. J. and Marianetti, C. A., Density functional versus spin-density functional and the choice of correlated subspace in multivariable effective action theories of electronic structure, Phys. Rev. B 92, 035146 (2015).

Pavarini, E., Koch, E., Vollhardt, D. and Lichtenstein, A., The LDA+DMFT approach to strongly correlated materials: lecture notes of the autumn school, vol. 1 of Schriften des Forschungszentrums Jülich. Reihe Modeling and simulation, Forschungszentrum, Zentralbibliothek (2011).

Perdew, J. P., Burke, K. and Ernzerhof, M., Generalized Gradient Approximation Made Simple, Phys. Rev. Lett. 77, 3865 (1996). 
Perdew, J. P., Chevary, J. A., Vosko, S. H., Jackson, K. A., Pederson, M. R., Singh, D. J. and Fiolhais, C., Atoms, molecules, solids, and surfaces: Applications of the generalized gradient approximation for exchange and correlation, Phys. Rev. B 46, 6671 (1992).

Perdew, J. P., Parr, R. G., Levy, M. and Balduz, J. L., Density-Functional Theory for Fractional Particle Number: Derivative Discontinuities of the Energy, Phys. Rev. Lett. 49, 1691 (1982).

Perdew, J. P. and Wang, Y., Accurate and simple analytic representation of the electron-gas correlation energy, Phys. Rev. B 45, 13244 (1992).

Perdew, J. P. and Zunger, A., Self-interaction correction to density-functional approximations for many-electron systems, Phys. Rev. B 23, 5048 (1981).

Ramirez, A. P., Colossal magnetoresistance, J. Phys.: Condens. Matter 9, 8171 (1997).

Reimers, J. N. and Dahn, J. R., Electrochemical and In Situ X-Ray Diffraction Studies of Lithium Intercalation in $\mathrm{Li}_{x} \mathrm{CoO}_{2}$, J. Electrochem. Soc. 139, 2091 (1992).

Reynier, Y., Graetz, J., Swan-Wood, T., Rez, P., Yazami, R. and Fultz, B., Entropy of Li intercalation in $\mathrm{Li}_{x} \mathrm{CoO}_{2}$, Phys. Rev. B 70, 174304 (2004).

Rousse, G., Rodriguez-Carvajal, J., Patoux, S. and Masquelier, C., Magnetic Structures of the Triphylite $\mathrm{LiFePO}_{4}$ and of Its Delithiated Form $\mathrm{FePO}_{4}$, Chem. Mater. 15, 4082 (2003).

Sánchez, K., Palacios, P. and Wahnón, P., Electronic structure of bulk- and Na-intercalated $\mathrm{TiS}_{2}$ determined from a GGA $+U$ study with the Hubbard terms obtained ab initio, Phys. Rev. B 78, 235121 (2008).

Santoro, R. P. and Newnham, R. E., Antiferromagnetism in $\mathrm{LiFePO}_{4}$, Acta Crystallogr. 22, 344 (1967). 
Santoro, R. P., Segal, D. J. and Newnham, R. E., Magnetic properties of $\mathrm{LiCoPO}_{4}$ and $\mathrm{LiNiPO}_{4}$, J. Phys. Chem. Solids 27, 1192 (1966).

Scalise, E., Houssa, M., Pourtois, G., Afanasev, V. and Stesmans, A., Strain-induced semiconductor to metal transition in the two-dimensional honeycomb structure of $\mathrm{MoS}_{2}$, Nano Res. 5, 43 (2012).

Seo, D.-H., Urban, A. and Ceder, G., Calibrating transition-metal energy levels and oxygen bands in first-principles calculations: Accurate prediction of redox potentials and charge transfer in lithium transition-metal oxides, Phys. Rev. B 92, 115118 (2015).

Shi, S., Ouyang, C., Xiong, Z., Liu, L., Wang, Z., Li, H., Wang, D.-s., Chen, L. and Huang, $\mathrm{X}$., First-principles investigation of the structural, magnetic, and electronic properties of olivine $\mathrm{LiFePO}_{4}$, Phys. Rev. B 71, 144404 (2005).

Sivadas, N., Daniels, M. W., Swendsen, R. H., Okamoto, S. and Xiao, D., Magnetic ground state of semiconducting transition-metal trichalcogenide monolayers, Phys. Rev. B 91, 235425 (2015).

Stevanović, V., Lany, S., Zhang, X. and Zunger, A., Correcting density functional theory for accurate predictions of compound enthalpies of formation: Fitted elemental-phase reference energies, Phys. Rev. B 85, 115104 (2012).

Strobridge, F. C., Clément, R. J., Leskes, M., Middlemiss, D. S., Borkiewicz, O. J., Wiaderek, K. M., Chapman, K. W., Chupas, P. J. and Grey, C. P., Identifying the Structure of the Intermediate, $\mathrm{Li}_{2 / 3} \mathrm{CoPO}_{4}$, Formed during Electrochemical Cycling of $\mathrm{LiCoPO}_{4}$, Chem. Mater. 26, 6193 (2014).

Sun, X., Yao, T., Hu, Z., Guo, Y., Liu, Q., Wei, S. and Wu, C., In-situ Unravelling Structural Modulation across Charge-Density-Wave Transition in Vanadium Disulfide, Phys. Chem. Chem. Phys. 17, 13333 (2015). 
Tang, P. and Holzwarth, N. A. W., Electronic structure of $\mathrm{FePO}_{4}, \mathrm{LiFePO}_{4}$, and related materials, Phys. Rev. B 68, 165107 (2003).

Togo, A., Oba, F. and Tanaka, I., First-principles calculations of the ferroelastic transition between rutile-type and $\mathrm{CaCl}_{2}$-type $\mathrm{SiO}_{2}$ at high pressures, Phys. Rev. B 78, 134106 (2008).

Tsubokawa, I., On the Magnetic Properties of a $\mathrm{CrBr}_{3}$ Single Crystal, J. Phys. Soc. Jpn. 15, 1664 (1960).

Tsuda, T., Yasuoka, H., Kitaoka, Y. and Di Salvo, F. J., ${ }^{51} \mathrm{~V}$ NMR study of the phase transition in $1 \mathrm{~T}-\mathrm{VS}_{2}$, J. Magn. Magn. Mater. 3134, 1101 (1983).

van de Walle, A., Multicomponent multisublattice alloys, nonconfigurational entropy and other additions to the Alloy Theoretic Automated Toolkit, Calphad 33, 266 (2009).

van de Walle, A., Asta, M. and Ceder, G., The alloy theoretic automated toolkit: A user guide, Calphad 26, 539 (2002).

Van der Ven, A., Aydinol, M. K., Ceder, G., Kresse, G. and Hafner, J., First-principles investigation of phase stability in $\mathrm{Li}_{x} \mathrm{CoO}_{2}$, Phys. Rev. B 58, 2975 (1998).

van Elp, J., Wieland, J. L., Eskes, H., Kuiper, P., Sawatzky, G. A., de Groot, F. M. F. and Turner, T. S., Electronic structure of $\mathrm{CoO}$, Li-doped $\mathrm{CoO}$, and $\mathrm{LiCoO}_{2}$, Phys. Rev. B 44, 6090 (1991).

van Laar, B. and Ijdo, D., Preparation, crystal structure, and magnetic structure of $\mathrm{LiCrS}_{2}$ and $\mathrm{LiVS}_{2}$, J. Solid State Chem. 3, 590 (1971).

Visscher, P. B., Phase separation instability in the Hubbard model, Phys. Rev. B 10, 943 (1974). 
Vosko, S. H., Wilk, L. and Nusair, M., Accurate spin-dependent electron liquid correlation energies for local spin density calculations: a critical analysis, Can. J. Phys. 58, 1200 (1980).

Werner, P., Comanac, A., de Medici, L., Troyer, M. and Millis, A. J., Continuous-Time Solver for Quantum Impurity Models, Phys. Rev. Lett. 97, 076405 (2006).

Werner, P. and Millis, A. J., Hybridization expansion impurity solver: General formulation and application to Kondo lattice and two-orbital models, Phys. Rev. B 74, 155107 (2006).

Whittingham, M. S., Lithium Batteries and Cathode Materials, Chem. Rev. 104, 4271 (2004).

Wildes, A. R., Roessli, B., Lebech, B. and Godfrey, K. W., Spin waves and the critical behaviour of the magnetization in $\mathrm{MnPS}_{3}$, J. Phys.: Condens. Matter 10, 6417 (1998).

Wilson, J. and Yoffe, A., The transition metal dichalcogenides discussion and interpretation of the observed optical, electrical and structural properties, Adv. Phys. 18, 193 (1969).

Wolverton, C. and Zunger, A., First-Principles Prediction of Vacancy Order-Disorder and Intercalation Battery Voltages in $\mathrm{Li}_{x} \mathrm{CoO}_{2}$, Phys. Rev. Lett. 81, 606 (1998).

Xu, Z., Joshi, Y. V., Raman, S. and Kitchin, J. R., Accurate electronic and chemical properties of $3 \mathrm{~d}$ transition metal oxides using a calculated linear response $U$ and a DFT $+U(\mathrm{~V})$ method, J. Chem. Phys. 142, 144701 (2015).

Yamada, A., Chung, S. C. and Hinokuma, K., Optimized $\mathrm{LiFePO}_{4}$ for Lithium Battery Cathodes, J. Electrochem. Soc. 148, A224 (2001).

Yamada, A., Koizumi, H., Nishimura, S.-i., Sonoyama, N., Kanno, R., Yonemura, M., Nakamura, T. and Kobayashi, Y., Room-temperature miscibility gap in $\mathrm{Li}_{x} \mathrm{FePO}_{4}$, Nat. Mater. 5, 357 (2006). 
Yamada, A., Koizumi, H., Sonoyama, N. and Kanno, R., Phase Change in $\mathrm{Li}_{x} \mathrm{FePO}_{4}$, Electrochem. Solid-State Lett. 8, A409 (2005).

Zaghib, K., Mauger, A., Goodenough, J. B., Gendron, F. and Julien, C. M., Electronic, Optical, and Magnetic Properties of $\mathrm{LiFePO}_{4}$ : Small Magnetic Polaron Effects, Chem. Mater. 19, 3740 (2007).

Zhang, F. C. and Rice, T. M., Effective hamiltonian for the superconducting Cu oxides, Phys. Rev. B 37, 3759 (1988).

Zhang, H., Liu, L.-M. and Lau, W.-M., Dimension-dependent phase transition and magnetic properties of $\mathrm{VS}_{2}$, J. Mater. Chem. A 1, 10821 (2013).

Zhang, P., Luo, W., Crespi, V. H., Cohen, M. L. and Louie, S. G., Doping effects on the electronic and structural properties of $\mathrm{CoO}_{2}$ : An LSDA+U study, Phys. Rev. B 70, 085108 (2004).

Zhong, M., Li, Y., Xia, Q., Meng, X., Wu, F. and Li, J., Ferromagnetism in $\mathrm{VS}_{2}$ nanostructures: Nanoflowers versus ultrathin nanosheets, Mater. Lett. 124, 282 (2014).

Zhou, F., Cococcioni, M., Marianetti, C. A., Morgan, D. and Ceder, G., First-principles prediction of redox potentials in transition-metal compounds with $\mathrm{LDA}+U$, Phys. Rev. B 70, 235121 (2004).

Zhou, F., Kang, K., Maxisch, T., Ceder, G. and Morgan, D., The electronic structure and band gap of $\mathrm{LiFePO}_{4}$ and $\mathrm{LiMnPO}_{4}$, Solid State Comm. 132, 181 (2004).

Zhou, F., Marianetti, C. A., Cococcioni, M., Morgan, D. and Ceder, G., Phase separation in $\mathrm{Li}_{x} \mathrm{FePO}_{4}$ induced by correlation effects, Phys. Rev. B 69, 201101 (2004).

Zhou, F., Maxisch, T. and Ceder, G., Configurational Electronic Entropy and the Phase Diagram of Mixed-Valence Oxides: The Case of $\mathrm{Li}_{x} \mathrm{FePO}_{4}$, Phys. Rev. Lett. 97, 155704 (2006). 
Zhuang, H. L., Xie, Y., Kent, P. R. C. and Ganesh, P., Computational discovery of ferromagnetic semiconducting single-layer $\mathrm{CrSnTe}_{3}$, Phys. Rev. B 92, 035407 (2015).

Zunger, A., Wei, S.-H., Ferreira, L. G. and Bernard, J. E., Special quasirandom structures, Phys. Rev. Lett. 65, 353 (1990). 